\title{
Stereocontrolled Synthesis of Bispirooxindole-Based Hexahydroxanthones with Five Contiguous Stereocenters
}

\author{
Xiong-Li Liu, ${ }^{*},+\star$ Gen Zhou, ${ }^{\star}$ Yi Gong, ${ }^{\star}$ Zhen Yao, ${ }^{\star}$ Xiong Zuo, ${ }^{\star}$ Wen-Hui Zhang, ${ }^{\star}$ and Ying \\ Zhou $^{*}, \dagger, *$ \\ $\dagger$ College of Pharmaceutical Sciences, Guizhou University of Chinese Medicine, Guiyang, \\ Guizhou 550025, China. \\ $\$$ Guizhou Engineering Center for Innovative Traditional Chinese Medicine and Ethnic Medicine, \\ Guizhou University, Guiyang, Guizhou 550025, China. \\ E-mail: xlliu1@ gzu.edu.cn (X.L. Liu) and zhouy@ gzu.edu.cn (Y. Zhou)
}

\section{Table of Contents}

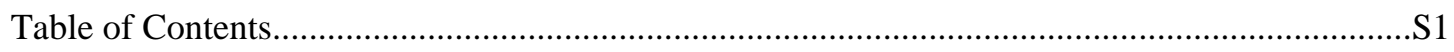

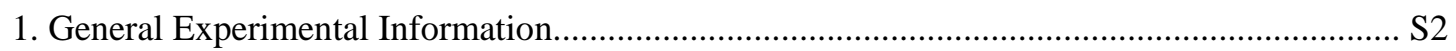

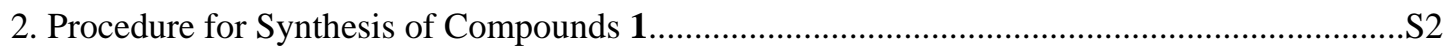

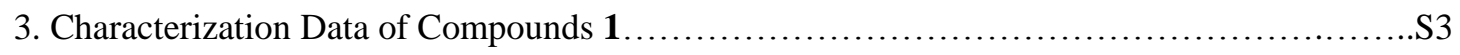

4. Table S1: Optimization of Reaction Conditions for Synthesis of Compound 3a...............S6

5. Typical Experimental Procedures for Catalytic Asymmetric Synthesis of Compounds $3 \ldots \ldots$...S7

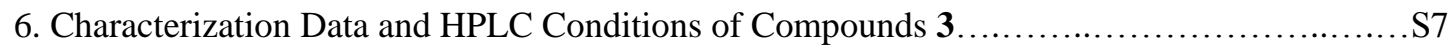

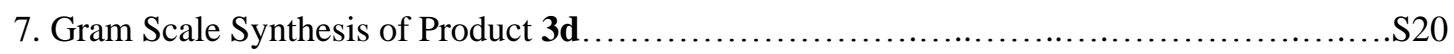

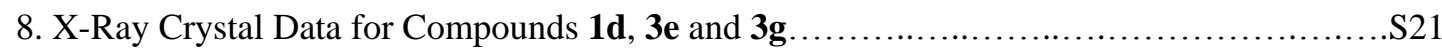

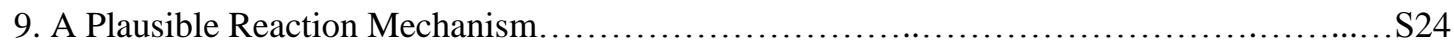

10. General Experimental Procedures for In Vitro Cytotoxicity Assay.........................S24

11. General Experimental Procedures for Flow Cytometry Analysis of Apoptotic Analysis.....S25

12. General Experimental Procedures for AO-EB Double Staining ............................S25

13. Figure S1: Compound 3a Induced Apoptosis of K562 Cells...............................S26

14. The Copies of ${ }^{1} \mathrm{H}$ NMR, ${ }^{13} \mathrm{C}$ NMR and HPLC Spectra for Compounds $\mathbf{1}, \mathbf{3}$ and $\mathbf{5} \ldots \ldots \ldots . . . \mathrm{S} 27$ 


\section{General Information}

Reactions were monitored by thin layer chromatography using UV light to visualize the course of reaction. Purification of reaction products was carried out by flash chromatography on silica gel. Chemical yields refered to pure isolated substances. The ee values were determined by chiral HPLC analysis. The d.r. values were determined by ${ }^{1} \mathrm{H}-\mathrm{NMR}$ analysis. ${ }^{1} \mathrm{H}$ and ${ }^{13} \mathrm{CNMR}$ spectra were obtained using a Bruker DPX-400 or DPX-500 spectrometer. ${ }^{1} \mathrm{H}$ NMR chemical shifts are reported in $\mathrm{ppm}(\delta)$ relative to tetramethylsilane (TMS) with the solvent resonance employed as the internal standard. Data are reported as follows: chemical shift, multiplicity $(\mathrm{s}=$ singlet, $\mathrm{d}=$ doublet, $\mathrm{t}=$ triplet, $\mathrm{m}=$ multiplet $)$, coupling constants $(\mathrm{Hz})$ and integration. ${ }^{13} \mathrm{C}$ NMR chemical shifts are reported in ppm $(\delta)$ from tetramethylsilane (TMS) with the solvent resonance as the internal standard. Optical rotations were measured with a polarimer with the solvent indicated. Melting points were measured on an electrothermal digital melting point apparatus.

\section{Procedure for the Synthesis of Compounds $1^{1-3}$}

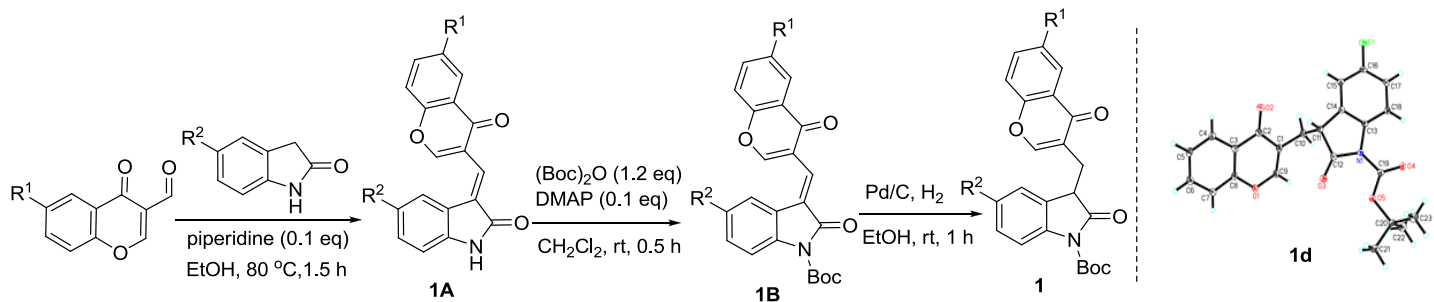

Chromone-3-carboxaldehyde $(10.5 \mathrm{mmol})$ and piperidine $(1.0 \mathrm{mmol})$ were added to a suspension of oxindole $(10.0 \mathrm{mmol})$ in ethanol $(50.0 \mathrm{~mL})$. The solution was heated at $80{ }^{\circ} \mathrm{C}$ for $1.5 \mathrm{~h}$. The reaction was allowed to cool to room temperature. The precipitate was filtered, washed with ethanol and dried to afford the product $\mathbf{1 A}$ as a red solid.

Compound $1 \mathrm{~A}(5.0 \mathrm{mmol})$ was dissolved in $\mathrm{CH}_{2} \mathrm{Cl}_{2}(50.0 \mathrm{~mL})$. To this solution were added DMAP $(0.5 \mathrm{mmol})$ and $(\mathrm{Boc})_{2} \mathrm{O}(6.0 \mathrm{mmol}, 1.2 \mathrm{eq})$ at room temperature, and then the mixture was stirred for $0.5 \mathrm{~h}$. The reaction mixture was diluted with ethyl acetate, and then quenched with saturated aqueous $\mathrm{NH}_{4} \mathrm{Cl}$. The aqueous layer was extracted with ethyl acetate, and the combined organic layers were washed with water and brine and then dried over $\mathrm{Na}_{2} \mathrm{SO}_{4}$. After the removal of solvent, purification by flash column chromatography ( hexane : ethyl acetate $=5: 1$ ) was carried out to give compound $\mathbf{1 B}$ as a red solid. 
Compound 1B $(3.0 \mathrm{mmol})$ was dissolved in $\mathrm{EtOH}(60.0 \mathrm{~mL}) .5 \% \mathrm{Pd} / \mathrm{C}$ (dry powder, $500.0 \mathrm{mg}$ ) was added to this solution, and the resulting mixture was stirred under hydrogen atmosphere (balloon) for $1 \mathrm{~h}$ at room temperature. The reaction mixture was passed through celite to remove $\mathrm{Pd} / \mathrm{C}$, and the residue was washed with EtOH. After the removal of solvent, the crude product was purified by flash column chromatography ( hexane : ethyl acetate $=10: 1$ ) to give $\mathbf{1}$ as a white solid.

\section{Characterization Data of Compounds 1}

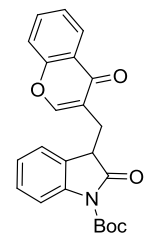

1a: White solid, m.p. $120.1-121.6{ }^{\circ} \mathrm{C} ; 2.19$ g, overall yield $56 \% ;{ }^{1} \mathrm{H} \mathrm{NMR}\left(\mathrm{CDCl}_{3}, 500 \mathrm{MHz}\right) \delta$ : $1.56(\mathrm{~s}, 9 \mathrm{H}), 2.91-2.99(\mathrm{~m}, 1 \mathrm{H}), 3.96-3.99(\mathrm{~m}, 1 \mathrm{H}), 7.03-7.06(\mathrm{~m}, 1 \mathrm{H}), 7.19-7.22(\mathrm{~m}, 2 \mathrm{H})$, 7.32-7.35 (m, 1H), $7.38(\mathrm{~d}, J=8.5 \mathrm{~Hz}, 1 \mathrm{H}), 7.58-7.61(\mathrm{~m}, 1 \mathrm{H}), 7.70(\mathrm{~d}, J=8.5 \mathrm{~Hz}, 1 \mathrm{H}), 7.78(\mathrm{~s}$, $1 \mathrm{H}), 8.14-8.16(\mathrm{~m}, 1 \mathrm{H}) ;{ }^{13} \mathrm{C} \mathrm{NMR}\left(\mathrm{CDCl}_{3}, 125 \mathrm{MHz}\right) \delta: 28.1,28.3,44.0,84.4,114.8,118.2,120.6$, $123.8,124.3,124.5,125.2,125.8,127.4,128.3,133.6,139.8,149.3,154.0,156.6,175.7,177.5$; HRMS (ESI-TOF) m/z: Calcd. for $\mathrm{C}_{23} \mathrm{H}_{21} \mathrm{NNaO}_{5}[\mathrm{M}+\mathrm{Na}]^{+}$: 414.1312; Found: 414.1316.

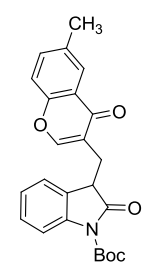

1b: White solid, m.p. $126.4-128.0{ }^{\circ} \mathrm{C} ; 1.94$ g, overall yield $48 \% ;{ }^{1} \mathrm{H}$ NMR $\left(\mathrm{CDCl}_{3}, 500 \mathrm{MHz}\right) \delta$ : $1.62(\mathrm{~s}, 9 \mathrm{H}), 2.43(\mathrm{~s}, 3 \mathrm{H}), 2.90-2.95(\mathrm{~m}, 1 \mathrm{H}), 3.01-3.07(\mathrm{~m}, 1 \mathrm{H})$, 4.01-4.05 (m, 1H), 7.07-7.11 (m, 1H), 7.23-7.27 (m, 2H), 7.33 (d, J = 8.4 Hz, 1H), 7.44-7.46 (m, 1H), 7.74-7.77 (m, 1H), $7.80(\mathrm{~s}$, 1H), $7.97(\mathrm{~s}, 1 \mathrm{H}) ;{ }^{13} \mathrm{C} \mathrm{NMR}\left(\mathrm{CDCl}_{3}, 125 \mathrm{MHz}\right) \delta: 21.1,28.2,28.4,44.0,84.4,114.9,118.0,120.4$, $123.5,124.3,124.6,125.1,127.5,128.3,135.0,135.2,139.9,149.3,154.0,154.9,175.8,177.7$; HRMS (ESI-TOF) m/z: Calcd. for $\mathrm{C}_{24} \mathrm{H}_{23} \mathrm{NNaO}_{5}[\mathrm{M}+\mathrm{Na}]^{+}$: 428.1468; Found: 428.1464. 


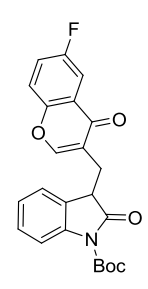

1c: White solid, m.p. $161.2-162.6{ }^{\circ} \mathrm{C} ; 2.29$ g, overall yield $56 \% ;{ }^{1} \mathrm{H} \mathrm{NMR}\left(\mathrm{CDCl}_{3}, 400 \mathrm{MHz}\right) \delta$ : $1.62(\mathrm{~s}, 9 \mathrm{H}), 2.98-3.01(\mathrm{~m}, 2 \mathrm{H}), 3.99-4.02(\mathrm{~m}, 1 \mathrm{H}), 7.09-7.13(\mathrm{~m}, 1 \mathrm{H}), 7.25-7.29(\mathrm{~m}, 2 \mathrm{H})$, 7.35-7.40 (m, 1H), 7.43-7.47 (m, 1H), $7.75(\mathrm{~d}, J=8.0 \mathrm{~Hz}, 1 \mathrm{H}), 7.81-7.83(\mathrm{~m}, 1 \mathrm{H}), 7.85(\mathrm{~s}, 1 \mathrm{H})$; ${ }^{13} \mathrm{C} \mathrm{NMR}\left(\mathrm{CDCl}_{3}, 100 \mathrm{MHz}\right) \delta: 28.2,43.9,84.5,110.6\left(\mathrm{~d}, J_{C F}=24.5 \mathrm{~Hz}\right), 114.9,120.1,120.4(\mathrm{~d}$, $\left.J_{C F}=8.1 \mathrm{~Hz}\right), 122.0\left(\mathrm{~d}, J_{C F}=25.0 \mathrm{~Hz}\right), 124.4,124.5,124.9,125.0,127.3,128.4,139.8,149.3$, $152.8,154.3,159.6\left(\mathrm{~d}, J_{C F}=245.2 \mathrm{~Hz}\right), 175.8,176.9 ;{ }^{19} \mathrm{~F} \mathrm{NMR}\left(\mathrm{CDCl}_{3}, 376 \mathrm{MHz}\right) \delta:-115.09$; HRMS (ESI-TOF) m/z: Calcd. for $\mathrm{C}_{23} \mathrm{H}_{20} \mathrm{FNNaO}_{5}$ [M+Na] $]^{+}$: 432.1218; Found: 432.1222 .

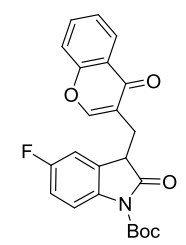

1d: White solid, m.p. $140.3-141.5{ }^{\circ} \mathrm{C} ; 2.33 \mathrm{~g}$, overall yield $57 \%$; ${ }^{1} \mathrm{H} \mathrm{NMR}\left(\mathrm{CDCl}_{3}, 500 \mathrm{MHz}\right) \delta$ : 1.55 (s, 9H), 2.87-2.97 (m, 2H), 3.96-3.99 (m, 1H), 6.87-6.91 (m, 1H), $6.96(\mathrm{~d}, J=7.0 \mathrm{~Hz}, 1 \mathrm{H})$, 7.31-7.33 (m, 1H), $7.38(\mathrm{~d}, J=8.5 \mathrm{~Hz}, 1 \mathrm{H}), 7.57-7.61(\mathrm{~m}, 1 \mathrm{H}), 7.67-7.70(\mathrm{~m}, 1 \mathrm{H}), 7.79(\mathrm{~s}, 1 \mathrm{H})$, $8.14(\mathrm{~d}, J=7.5 \mathrm{~Hz}, 1 \mathrm{H}) ;{ }^{13} \mathrm{C} \mathrm{NMR}\left(\mathrm{CDCl}_{3}, 125 \mathrm{MHz}\right) \delta: 28.1,28.3,44.1,84.5,112.0\left(\mathrm{~d}, J_{C F}=\right.$ $23.8 \mathrm{~Hz}), 114.7\left(\mathrm{~d}, J_{C F}=22.5 \mathrm{~Hz}\right), 116.1,118.2,120.4,123.8,125.2,125.8,129.2,129.3,133.7$, $135.8,149.2,154.0,156.6,159.8\left(\mathrm{~d}, J_{C F}=242.5 \mathrm{~Hz}\right), 175.2,177.5 ;{ }^{19} \mathrm{~F} \mathrm{NMR}\left(\mathrm{CDCl}_{3}, 470 \mathrm{MHz}\right)$ $\delta$ : -117.84; HRMS (ESI-TOF) m/z: Calcd. for $\mathrm{C}_{23} \mathrm{H}_{20} \mathrm{FNNaO}_{5}[\mathrm{M}+\mathrm{Na}]^{+}$: 432.1218; Found: 432.1223.

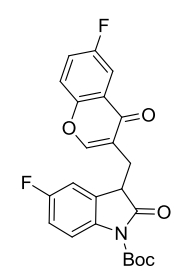

1e: White solid, m.p. $131.2-132.8{ }^{\circ} \mathrm{C} ; 1.84 \mathrm{~g}$, overall yield $43 \%$; ${ }^{1} \mathrm{H} \mathrm{NMR}\left(\mathrm{CDCl}_{3}, 400 \mathrm{MHz}\right) \delta$ : $1.60(\mathrm{~s}, 9 \mathrm{H}), 2.96(\mathrm{~d}, J=6.4 \mathrm{~Hz}, 2 \mathrm{H}), 3.98-4.02(\mathrm{~m}, 1 \mathrm{H}), 6.93-6.97(\mathrm{~m}, 1 \mathrm{H}), 7.02(\mathrm{~d}, J=6.8 \mathrm{~Hz}$, 1H), 7.35-7.39 (m, 1H), 7.44-7.47 (m, 1H), 7.72-7.76 (m, 1H), $7.80(\mathrm{~d}, J=7.6 \mathrm{~Hz}, 1 \mathrm{H}), 7.86(\mathrm{~s}$, 
$1 \mathrm{H}) ;{ }^{13} \mathrm{C} \mathrm{NMR}\left(\mathrm{CDCl}_{3}, 100 \mathrm{MHz}\right) \delta: 28.1,28.2,44.0,84.7,110.6\left(\mathrm{~d}, J_{C F}=23.1 \mathrm{~Hz}\right), 112.0\left(\mathrm{~d}, J_{C F}\right.$ $=24.0 \mathrm{~Hz}), 114.9\left(\mathrm{~d}, J_{C F}=22.3 \mathrm{~Hz}\right), 116.2,119.8,120.4,120.5,122.1\left(\mathrm{~d}, J_{C F}=26.1 \mathrm{~Hz}\right), 124.9(\mathrm{~d}$, $\left.J_{C F}=7.1 \mathrm{~Hz}\right), 129.1\left(\mathrm{~d}, J_{C F}=8.3 \mathrm{~Hz}\right), 135.8,149.2,152.8,154.3,159.6\left(\mathrm{~d}, J_{C F}=246.8 \mathrm{~Hz}\right)$, $159.8\left(\mathrm{~d}, J_{C F}=242.1 \mathrm{~Hz}\right), 175.2,176.8,176.9 ;{ }^{19} \mathrm{~F} \mathrm{NMR}\left(\mathrm{CDCl}_{3}, 376 \mathrm{MHz}\right) \delta:-114.91,-117.75$; HRMS (ESI-TOF) m/z: Calcd. for $\mathrm{C}_{23} \mathrm{H}_{19} \mathrm{~F}_{2} \mathrm{NNaO}_{5}$ [M+Na] $]^{+}$: 450.1124; Found: 450.1126.

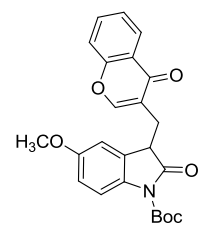

1f: White solid, m.p. $125.1-126.3{ }^{\circ} \mathrm{C} ; 1.52 \mathrm{~g}$, overall yield $36 \% ;{ }^{1} \mathrm{H} \mathrm{NMR}\left(\mathrm{CDCl}_{3}, 400 \mathrm{MHz}\right) \delta$ : $1.56(\mathrm{~s}, 9 \mathrm{H}), 2.92-2.94(\mathrm{~m}, 2 \mathrm{H}), 3.70$ (s, 3H), 3.93-3.95 (m, 1H), 6.73-6.75 (m, 1H), $6.82(\mathrm{~s}, 1 \mathrm{H})$, 7.33-7.39 (m, 2H), 7.60-7.62 (m, 2H), $7.80(\mathrm{~s}, 1 \mathrm{H}), 8.15(\mathrm{~s}, 1 \mathrm{H}) ;{ }^{13} \mathrm{C} \mathrm{NMR}\left(\mathrm{CDCl}_{3}, 100 \mathrm{MHz}\right) \delta$ : $28.1,28.2,44.2,55.6,84.2,110.4,113.4,115.7,118.2,120.6,123.8,125.1,125.8,128.6,133.1$, 133.6, 149.3, 154.0, 156.5, 156.7, 175.7, 177.6; HRMS (ESI-TOF) m/z: Calcd. for $\mathrm{C}_{24} \mathrm{H}_{23} \mathrm{NNaO}_{6}$ $[\mathrm{M}+\mathrm{Na}]^{+}:$444.1418; Found: 444.1423. 


\section{Table S1: Optimization of Reaction Conditions for Synthesis of Compound 3a ${ }^{a}$}
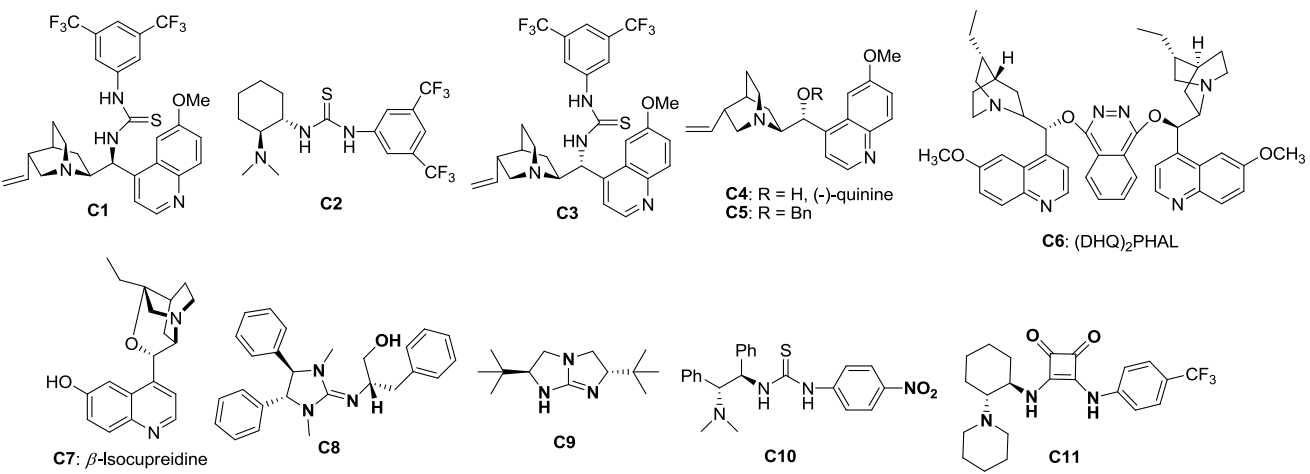

C7: $\beta$-Isocupreidine

$\mathrm{C8}-\mathrm{C} 10$ c11

\begin{tabular}{|c|c|c|c|c|c|c|c|c|}
\hline entry & $\mathrm{R}^{1}$ & $\mathrm{R}^{2}$ & catalyst & solvent & time[d] & yield $[\%]^{b}$ & d.r. ${ }^{c}$ & ee $[\%]^{d}$ \\
\hline 1 & Boc & Ac & C1 & $\mathrm{CH}_{2} \mathrm{Cl}_{2}$ & 3 & 33 & $4: 1$ & $83(+)$ \\
\hline 2 & Boc & Ac & $\mathrm{C} 2$ & $\mathrm{CH}_{2} \mathrm{Cl}_{2}$ & 3 & 23 & $8: 1$ & $41(+)$ \\
\hline 3 & Boc & Ac & $\mathrm{C3}$ & $\mathrm{CH}_{2} \mathrm{Cl}_{2}$ & 3 & trace & - & - \\
\hline 4 & Boc & Ac & $\mathrm{C4}$ & $\mathrm{CH}_{2} \mathrm{Cl}_{2}$ & 3 & 40 & $1: 1$ & $9(+)$ \\
\hline 5 & Boc & Ac & C5 & $\mathrm{CH}_{2} \mathrm{Cl}_{2}$ & 3 & 20 & $2: 3$ & $13(-)$ \\
\hline 6 & Boc & $\mathrm{Ac}$ & C6 & $\mathrm{CH}_{2} \mathrm{Cl}_{2}$ & 3 & trace & - & - \\
\hline 7 & Boc & Ac & C7 & $\mathrm{CH}_{2} \mathrm{Cl}_{2}$ & 3 & 80 & $15: 1$ & $6(+)$ \\
\hline 8 & Boc & Ac & C8 & $\mathrm{CH}_{2} \mathrm{Cl}_{2}$ & 3 & 25 & $19: 1$ & $5(+)$ \\
\hline 9 & Boc & Ac & C9 & $\mathrm{CH}_{2} \mathrm{Cl}_{2}$ & 3 & trace & - & - \\
\hline 10 & Boc & Ac & $\mathrm{C} 10$ & $\mathrm{CH}_{2} \mathrm{Cl}_{2}$ & 3 & 28 & $3: 1$ & $67(+)$ \\
\hline 11 & Boc & Ac & C11 & $\mathrm{CH}_{2} \mathrm{Cl}_{2}$ & 3 & 16 & $2: 3$ & $38(-)$ \\
\hline 12 & Boc & Ac & C1 & $\mathrm{CHCl}_{3}$ & 3 & 38 & $5: 1$ & $82(+)$ \\
\hline 13 & Boc & Ac & $\mathrm{C1}$ & toluene & 3 & 27 & $4: 1$ & $65(+)$ \\
\hline 14 & Boc & Ac & $\mathrm{C1}$ & $\mathrm{Et}_{2} \mathrm{O}$ & 3 & 60 & $5: 1$ & $82(+)$ \\
\hline $15^{e}$ & Boc & Ac & C1 & $\mathrm{Et}_{2} \mathrm{O}$ & 7 & 42 & $5: 1$ & $92(+)$ \\
\hline $16^{f}$ & Boc & Ac & $\mathrm{C} 1$ & $\mathrm{Et}_{2} \mathrm{O}$ & 3 & 54 & $4: 1$ & $85(+)$ \\
\hline $17^{g}$ & Boc & Ac & C1 & $\mathrm{Et}_{2} \mathrm{O}$ & 3 & 46 & $5: 1$ & $90(+)$ \\
\hline $18^{g, h}$ & Boc & Ac & $\mathrm{C} 1$ & $\mathrm{Et}_{2} \mathrm{O}$ & 3 & 52 & $6: 1$ & $95(+)$ \\
\hline 19 & Boc & $\mathrm{Me}$ & $\mathrm{C1}$ & $\mathrm{Et}_{2} \mathrm{O}$ & 3 & $<10$ & - & - \\
\hline 20 & $\mathrm{Me}$ & $\mathrm{Me}$ & C1 & $\mathrm{Et}_{2} \mathrm{O}$ & 3 & $<10$ & - & - \\
\hline 21 & $\mathrm{H}$ & $\mathrm{Me}$ & C1 & $\mathrm{Et}_{2} \mathrm{O}$ & 3 & $<10$ & - & - \\
\hline
\end{tabular}

${ }^{a}$ Unless otherwise noted, the reactions were carried out with $\mathbf{1 a}(0.10 \mathrm{mmol}), \mathbf{2 a}(0.15 \mathrm{mmol})$, catalyst $(10 \mathrm{~mol} \%)$ in $1.0 \mathrm{~mL}$ solvent at $\mathrm{rt}$ for $3 \mathrm{~d} ;{ }^{b}$ Isolated yield; ${ }^{c}$ Determined by ${ }^{1} \mathrm{H}$-NMR analysis of the crude products; ${ }^{d}$ Determined by chiral HPLC analysis; ${ }^{e}$ Run in $2.5 \mathrm{~mL}$ of $\mathrm{Et}_{2} \mathrm{O}$ with $5 \mathrm{~mol} \%$ of catalyst $\mathbf{C 1}$ at $\mathrm{rt}$ for $7 \mathrm{~d} ;{ }^{f} \mathrm{Run}$ in $2.5 \mathrm{~mL}$ of $\mathrm{Et}_{2} \mathrm{O}$ with $15 \mathrm{~mol} \%$ of catalyst $\mathbf{C 1}$ at rt for $3 \mathrm{~d} ;{ }^{g}$ Run in $2.5 \mathrm{~mL}_{\text {of }} \mathrm{Et}_{2} \mathrm{O}$ with $10 \mathrm{~mol} \%$ of catalyst $\mathbf{C 1}$ at $\mathrm{rt}$ for $3 \mathrm{~d} ;{ }^{h} 60 \mathrm{Mg}$ of $5 \AA$ MS were used. 


\section{Typical Experimental Procedures for Catalytic Asymmetric Synthesis of Compounds 3}

In a sealed tube equipped with a magnetic stirring bar, to the mixture of oxindole-chromone synthon $1(0.10 \mathrm{mmol}), 5 \AA \mathrm{MS}(60 \mathrm{mg})$ and quinine-derived thiourea catalyst $\mathbf{C 1}(10 \mathrm{~mol} \%)$ in $2.5 \mathrm{~mL}$ of freshly distilled $\mathrm{Et}_{2} \mathrm{O}$ was added methyleneindolinone $2(0.15 \mathrm{mmol})$. The reaction mixture was stirred at rt for $3 \mathrm{~d}$ and was directly loaded onto a silica gel and purified by flash chromatography to give the desired product 3 , using hexane/EtOAc $(10 / 1, \mathrm{v} / \mathrm{v})$ as the eluent.

\section{Characterization Data and HPLC Conditions of Compounds 3}

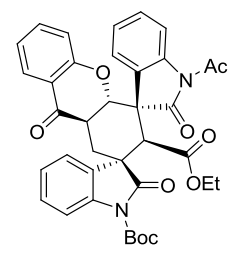

3'a: White solid, m.p. $122.1-123.7{ }^{\circ} \mathrm{C}$; $33.8 \mathrm{mg}$, yield 52\%; 95\% ee, $6: 1 \mathrm{dr},[\alpha]_{\mathrm{D}}{ }^{20}=+120.5(c 0.19$, $\mathrm{CH}_{2} \mathrm{Cl}_{2}$ ); The ee was determined by HPLC analysis using a Chiralpak IA column (90/10 hexane $/ i$-PrOH; flow rate: $\left.1.0 \mathrm{~mL} / \mathrm{min} ; \lambda=254 \mathrm{~nm} ; \tau_{\text {major }}=17.78 \mathrm{~min} ; \tau_{\text {minor }}=11.32 \mathrm{~min}\right) ;{ }^{1} \mathrm{H}$ $\operatorname{NMR}\left(\mathrm{CDCl}_{3}, 400 \mathrm{MHz}\right) \delta:$ 0.50-0.54 (m, 3H), $1.67(\mathrm{~s}, 9 \mathrm{H}), 2.46-2.52(\mathrm{~m}, 1 \mathrm{H}), 2.69-2.74(\mathrm{~m}, 1 \mathrm{H})$, $2.76(\mathrm{~s}, 3 \mathrm{H}), 3.16-3.22(\mathrm{~m}, 1 \mathrm{H}), 3.31-3.37(\mathrm{~m}, 1 \mathrm{H}), 3.85-3.94(\mathrm{~m}, 1 \mathrm{H}), 4.13(\mathrm{~s}, 1 \mathrm{H}), 5.89(\mathrm{~d}, J=$ $14.0 \mathrm{~Hz}, 1 \mathrm{H}), 6.68(\mathrm{~d}, J=8.4 \mathrm{~Hz}, 1 \mathrm{H}), 6.96-6.70(\mathrm{~m}, 1 \mathrm{H}), 7.17-7.21(\mathrm{~m}, 2 \mathrm{H}), 7.28-7.38(\mathrm{~m}, 4 \mathrm{H})$, 7.76-7.83 (m, 3H), $8.25(\mathrm{~d}, J=8.0 \mathrm{~Hz}, 1 \mathrm{H}) ;{ }^{13} \mathrm{C} \mathrm{NMR}\left(\mathrm{CDCl}_{3}, 100 \mathrm{MHz}\right) \delta: 13.0,26.9,28.3,31.5$, $38.9,47.6,54.0,55.9,61.4,80.9,84.7,115.2,115.7,117.9,120.5,121.8,122.1,125.1,125.8$, $126.3,127.3,128.0,129.2,129.3,132.5,136.1,139.5,141.4,149.1,160.1,167.6,170.8,178.8$, 179.5, 192.5; HRMS (ESI-TOF) m/z: Calcd. for $\mathrm{C}_{37} \mathrm{H}_{34} \mathrm{~N}_{2} \mathrm{NaO}_{9}[\mathrm{M}+\mathrm{Na}]^{+}$: 673.2157; Found: 673.2156 .

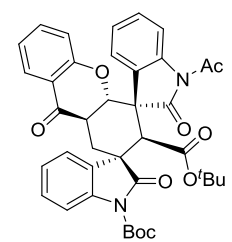

3a: White solid, m.p. $185.4-187.0{ }^{\circ} \mathrm{C} ; 46.8 \mathrm{mg}$, yield 69\%; 94\% ee, $>20: 1 \mathrm{dr},[\alpha]_{\mathrm{D}}{ }^{20}=+128.1(\mathrm{c}$ 0.43, $\mathrm{CH}_{2} \mathrm{Cl}_{2}$ ); The ee was determined by HPLC analysis using a Chiralpak IA column (90/10 hexane $/ i$-PrOH; flow rate: $1.0 \mathrm{~mL} / \mathrm{min} ; \lambda=254 \mathrm{~nm} ; \tau_{\text {major }}=12.55 \mathrm{~min} ; \tau_{\text {minor }}=9.13 \mathrm{~min}$ ); ${ }^{1} \mathrm{H}$ 
$\operatorname{NMR}\left(\mathrm{CDCl}_{3}, 400 \mathrm{MHz}\right) \delta: 0.66(\mathrm{~s}, 9 \mathrm{H}), 1.66(\mathrm{~s}, 9 \mathrm{H}), 2.45-2.53(\mathrm{~m}, 1 \mathrm{H}), 2.67-2.73(\mathrm{~m}, 1 \mathrm{H}), 2.74$ (s. 3H), 3.87-3.95 (m, 1H), $4.06(\mathrm{~s}, 1 \mathrm{H}), 5.88(\mathrm{~d}, J=14.4 \mathrm{~Hz}, 1 \mathrm{H}), 6.68(\mathrm{~d}, J=8.0 \mathrm{~Hz}, 1 \mathrm{H})$, 6.94-6.98 (m, 1H), 7.16-7.21 (m, 2H), 7.27-7.37 (m, 4H), 7.76-7.82 (m, 2H), 7.85-7.87 (m, 1H), $8.27(\mathrm{~d}, J=8.0 \mathrm{~Hz}, 1 \mathrm{H}) ;{ }^{13} \mathrm{C} \mathrm{NMR}\left(\mathrm{CDCl}_{3}, 100 \mathrm{MHz}\right) \delta: 26.8,27.0,28.3,31.8,38.9,47.6,54.1$, $56.9,80.9,82.6,84.7,115.1,115.8,118.0,120.4,122.0,125.0,125.7,126.9,127.2,128.0,129.0$, $129.3,132.8,136.1,139.4,141.5,149.1,160.1,166.2,170.8,178.8,179.5,192.7$; HRMS (ESI-TOF) m/z: Calcd. for $\mathrm{C}_{39} \mathrm{H}_{38} \mathrm{~N}_{2} \mathrm{NaO}_{9}[\mathrm{M}+\mathrm{Na}]^{+}$: 701.2470; Found: 701.2474.

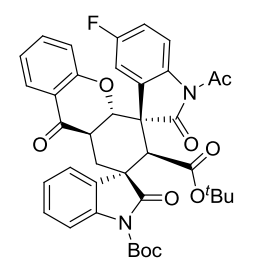

3b: White solid, m.p. $225.6-227.1{ }^{\circ} \mathrm{C}$; $63.3 \mathrm{mg}$, yield 91\%; 99\% ee, >20:1 dr, $[\alpha]_{\mathrm{D}}{ }^{20}=+145.8(c$ 0.33, $\mathrm{CH}_{2} \mathrm{Cl}_{2}$ ); The ee was determined by HPLC analysis using a Chiralpak IA column (98/2 hexane $/ i-\mathrm{PrOH}$; flow rate: $\left.1.0 \mathrm{~mL} / \mathrm{min} ; \lambda=254 \mathrm{~nm} ; \tau_{\text {major }}=19.79 \mathrm{~min} ; \tau_{\text {minor }}=26.96 \mathrm{~min}\right) ;{ }^{1} \mathrm{H}$ NMR $\left(\mathrm{CDCl}_{3}, 400 \mathrm{MHz}\right) \delta: 0.71(\mathrm{~s}, 9 \mathrm{H}), 1.65(\mathrm{~s}, 9 \mathrm{H}), 2.46-2.53(\mathrm{~m}, 1 \mathrm{H}), 2.65-2.71(\mathrm{~m}, 1 \mathrm{H}), 2.73$ (s, 3H), 3.86-3.95 (m, 1H), $4.04(\mathrm{~s}, 1 \mathrm{H}), 5.88(\mathrm{~d}, J=14.4 \mathrm{~Hz}, 1 \mathrm{H}), 6.69-6.71(\mathrm{~m}, 1 \mathrm{H}), 6.97-7.07$ (m, 2H), 7.17-7.21 (m, 1H), 7.29-7.33 (m, 2H), 7.36-7.41 (m, 1H), 7.63-7.66 (m, 1H), 7.81-7.85 $(\mathrm{m}, 2 \mathrm{H}), 8.26-8.30(\mathrm{~m}, 1 \mathrm{H}) ;{ }^{13} \mathrm{C} \mathrm{NMR}\left(\mathrm{CDCl}_{3}, 100 \mathrm{MHz}\right) \delta: 26.8,27.0,28.2,31.8,38.8,47.6$, 54.3, 56.9, 80.8, 82.9, 84.8, 114.8, 115.1, $115.8\left(\mathrm{~d}, J_{C F}=22.5 \mathrm{~Hz}\right), 117.2\left(\mathrm{~d}, J_{C F}=8.0 \mathrm{~Hz}\right), 117.9$, $120.5,121.7,122.2,125.1,127.3,129.1,130.0\left(\mathrm{~d}, J_{C F}=9.1 \mathrm{~Hz}\right), 132.5,136.2,137.6,139.6,149.2$, 160.0, $160.4\left(\mathrm{~d}, J_{C F}=243.3 \mathrm{~Hz}\right), 166.1,170.6,178.8,179.0,192.5 ;{ }^{19} \mathrm{~F} \mathrm{NMR}\left(\mathrm{CDCl}_{3}, 376 \mathrm{MHz}\right)$ $\delta$ : -115.64; HRMS (ESI-TOF) m/z: Calcd. for $\mathrm{C}_{39} \mathrm{H}_{37} \mathrm{FN}_{2} \mathrm{NaO}_{9}[\mathrm{M}+\mathrm{Na}]^{+}$: 719.2375; Found: 719.2378.

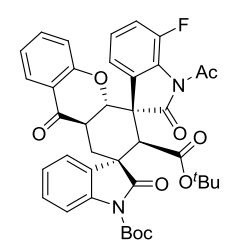

3c: White solid, m.p. $112.3-114.1{ }^{\circ} \mathrm{C} ; 60.5 \mathrm{mg}$, yield $87 \%$; $>99 \%$ ee, $>20: 1 \mathrm{dr},[\alpha]_{\mathrm{D}}{ }^{20}=+112.6(c$ $0.35, \mathrm{CH}_{2} \mathrm{Cl}_{2}$ ); The ee was determined by HPLC analysis using a Chiralpak IC column (95/5 hexane $/ i-\mathrm{PrOH}$; flow rate: $1.0 \mathrm{~mL} / \mathrm{min} ; \lambda=254 \mathrm{~nm} ; \tau_{\text {major }}=18.33 \mathrm{~min} ; \tau_{\text {minor }}=27.93 \mathrm{~min}$ ); ${ }^{1} \mathrm{H}$ 
$\operatorname{NMR}\left(\mathrm{CDCl}_{3}, 400 \mathrm{MHz}\right) \delta: 0.71(\mathrm{~s}, 9 \mathrm{H}), 1.66(\mathrm{~s}, 9 \mathrm{H}), 2.43-2.50(\mathrm{~m}, 1 \mathrm{H}), 2.66-2.72(\mathrm{~m}, 1 \mathrm{H}), 2.74$ (s, 3H), 3.81-3.90 (m, 1H), $4.06(\mathrm{~s}, 1 \mathrm{H}), 5.86(\mathrm{~d}, J=14.4 \mathrm{~Hz}, 1 \mathrm{H}), 6.68-6.71(\mathrm{~m}, 1 \mathrm{H}), 6.96-7.00$ (m, 1H), 7.10-7.22 (m, 3H), 7.28-7.39 (m, 3H), 7.65-7.67 (m, 1H), 7.76-7.82 (m, 2H); ${ }^{13} \mathrm{C}$ NMR $\left(\mathrm{CDCl}_{3}, 100 \mathrm{MHz}\right) \delta: 26.2,26.9,28.2,38.8,47.6,55.3,56.3,80.9,82.9,84.8,115.2,117.7,117.9$, 120.4, 121.6, 122.2, 122.7, 125.1, $126.8\left(\mathrm{~d}, J_{C F}=7.1 \mathrm{~Hz}\right), 127.2,127.6\left(\mathrm{~d}, J_{C F}=10.0 \mathrm{~Hz}\right), 129.0$, 131.6, 132.7, 136.2, 139.4, 149.0, $149.4\left(\mathrm{~d}, J_{C F}=252.0 \mathrm{~Hz}\right), 159.9,166.2,168.2,178.5,178.9$, 192.4; ${ }^{19} \mathrm{~F}$ NMR $\left(\mathrm{CDCl}_{3}, 376 \mathrm{MHz}\right) \quad \delta$ : -112.44 ; HRMS (ESI-TOF) $\mathrm{m} / \mathrm{z}$ : Calcd. for $\mathrm{C}_{39} \mathrm{H}_{37} \mathrm{FN}_{2} \mathrm{NaO}_{9}[\mathrm{M}+\mathrm{Na}]^{+}:$719.2375; Found: 719.2382.

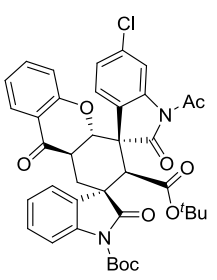

3d: White solid, m.p. $123.2-125.0{ }^{\circ} \mathrm{C} ; 58.3 \mathrm{mg}$, yield $82 \%$; >99\% ee, $>20: 1 \mathrm{dr},[\alpha]_{\mathrm{D}}{ }^{20}=+147.6(c$ 0.33, $\mathrm{CH}_{2} \mathrm{Cl}_{2}$ ); The ee was determined by HPLC analysis using a Chiralpak IC column (98/2 hexane $/ i-\mathrm{PrOH}$; flow rate: $1.0 \mathrm{~mL} / \mathrm{min} ; \lambda=254 \mathrm{~nm} ; \tau_{\text {major }}=13.97 \mathrm{~min} ; \tau_{\text {minor }}=25.11 \mathrm{~min}$ ); ${ }^{1} \mathrm{H}$ NMR (CDCl, $400 \mathrm{MHz}) \delta: 0.71(\mathrm{~s}, 9 \mathrm{H}), 1.66(\mathrm{~s}, 9 \mathrm{H}), 2.44-2.51(\mathrm{~m}, 1 \mathrm{H}), 2.65-2.72(\mathrm{~m}, 1 \mathrm{H}), 2.73$ (s, 3H), 3.84-3.92 (m, 1H), $4.03(\mathrm{~s}, 1 \mathrm{H}), 5.83(\mathrm{~d}, J=14.4 \mathrm{~Hz}, 1 \mathrm{H}), 6.69-6.71(\mathrm{~m}, 1 \mathrm{H}), 6.96-7.00$ (m, 1H), 7.16-7.21 (m, 2H), 7.28-7.32 (m, 2H), 7.36-7.40 (m, 1H), 7.77-7.83 (m, 3H), $8.35(\mathrm{~d}, J=$ $2.0 \mathrm{~Hz}, 1 \mathrm{H}) ;{ }^{13} \mathrm{C} \mathrm{NMR}\left(\mathrm{CDCl}_{3}, 100 \mathrm{MHz}\right) \delta: 26.8,26.9,28.2,31.8,38.8,47.6,53.9,56.7,80.8$, $82.9,84.8,115.2,116.4,117.9,120.4,121.6,122.2,125.1,125.7,126.5,127.3,127.9,129.1$, $132.7,135.1,136.2,139.4,142.3,149.0,160.0,166.1,170.6,178.9,179.1,192.5$; HRMS (ESI-TOF) m/z: Calcd. for $\mathrm{C}_{39} \mathrm{H}_{37} \mathrm{ClN}_{2} \mathrm{NaO}_{9}[\mathrm{M}+\mathrm{Na}]^{+}$: 735.2080; Found: 735.2086.

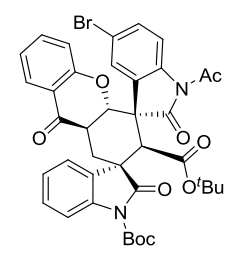

3e: White solid, m.p. $101.1-102.6{ }^{\circ} \mathrm{C} ; 67.3 \mathrm{mg}$, yield $89 \%$; $98 \%$ ee, $>20: 1 \mathrm{dr},[\alpha]_{\mathrm{D}}{ }^{20}=+110.9(c$ 0.43, $\mathrm{CH}_{2} \mathrm{Cl}_{2}$ ); The ee was determined by HPLC analysis using a Chiralpak IA column $(98 / 2$ hexane $/ i$-PrOH; flow rate: $\left.1.0 \mathrm{~mL} / \mathrm{min} ; \lambda=254 \mathrm{~nm} ; \tau_{\text {major }}=16.18 \mathrm{~min} ; \tau_{\text {minor }}=27.37 \mathrm{~min}\right) ;{ }^{1} \mathrm{H}$ NMR $\left(\mathrm{CDCl}_{3}, 400 \mathrm{MHz}\right) \delta: 0.72(\mathrm{~s}, 9 \mathrm{H}), 1.68(\mathrm{~s}, 9 \mathrm{H}), 2.54-2.57(\mathrm{~m}, 1 \mathrm{H}), 2.67-2.75(\mathrm{~m}, 1 \mathrm{H}), 2.75$ 
(s, 3H), 3.88-3.96 (m, 1H), $4.04(\mathrm{~s}, 1 \mathrm{H}), 5.93(\mathrm{~d}, J=14.4 \mathrm{~Hz}, 1 \mathrm{H}), 6.72(\mathrm{~d}, J=8.0 \mathrm{~Hz}, 1 \mathrm{H})$, 6.99-7.03 (m, 1H), 7.19-7.23 (m, 1H), 7.32-7.36 (m, 2H), 7.38-7.42 (m, 1H), 7.48-7.51 (m, 1H), 7.83-7.85 (m, 1H), $7.91(\mathrm{~d}, J=8.0 \mathrm{~Hz}, 1 \mathrm{H}), 8.01(\mathrm{~s}, 1 \mathrm{H}), 8.20(\mathrm{~d}, J=8.8 \mathrm{~Hz}, 1 \mathrm{H}) ;{ }^{13} \mathrm{C}$ NMR $\left(\mathrm{CDCl}_{3}, 100 \mathrm{MHz}\right) \delta: 26.7,28.2,31.7,38.7,47.5,54.1,57.2,80.6,83.1,84.6,115.0,117.4,117.8$, $118.5,120.4,121.7,122.1,125.0,127.2,129.1,130.0,130.2,132.2,136.1,139.6,140.5,149.4$, 159.9, 166.0, 170.5, 178.4, 192.4; HRMS (ESI-TOF) m/z: Calcd. for $\mathrm{C}_{39} \mathrm{H}_{37} \mathrm{BrN}_{2} \mathrm{NaO}_{9}[\mathrm{M}+\mathrm{Na}]^{+}$: 779.1575; Found: 779.1580.

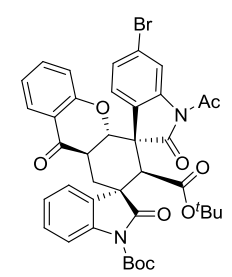

3f: White solid, m.p. $108.4-110.1{ }^{\circ} \mathrm{C} ; 60.5 \mathrm{mg}$, yield $80 \%$; >99\% ee, $>20: 1 \mathrm{dr},[\alpha]_{\mathrm{D}}{ }^{20}=+123.6(c$ 0.19, $\mathrm{CH}_{2} \mathrm{Cl}_{2}$ ); The ee was determined by HPLC analysis using a Chiralpak IC column (98/2 hexane $/ i$-PrOH; flow rate: $\left.1.0 \mathrm{~mL} / \mathrm{min} ; \lambda=254 \mathrm{~nm} ; \tau_{\text {major }}=14.12 \mathrm{~min} ; \tau_{\text {minor }}=25.44 \mathrm{~min}\right) ;{ }^{1} \mathrm{H}$ NMR $\left(\mathrm{CDCl}_{3}, 400 \mathrm{MHz}\right) \delta: 0.71(\mathrm{~s}, 9 \mathrm{H}), 1.66(\mathrm{~s}, 9 \mathrm{H}), 2.44-2.51(\mathrm{~m}, 1 \mathrm{H}), 2.65-2.71(\mathrm{~m}, 1 \mathrm{H}), 2.73$ (s, 3H), 3.84-3.92 (m, 1H), $4.03(\mathrm{~s}, 1 \mathrm{H}), 5.83(\mathrm{~d}, J=14.0 \mathrm{~Hz}, 1 \mathrm{H}), 6.70(\mathrm{~d}, J=8.4 \mathrm{~Hz}, 1 \mathrm{H})$, 6.97-7.00 (m, 1H), 7.17-7.20 (m, 1H), 7.28-7.40 (m, 4H), 7.75-7.82 (m, 3H), $8.50(\mathrm{~s}, 1 \mathrm{H}) ;{ }^{13} \mathrm{C}$ $\mathrm{NMR}\left(\mathrm{CDCl}_{3}, 100 \mathrm{MHz}\right) \delta: 26.8,26.9,28.2,31.8,38.8,47.6,54.0,56.6,80.7,83.0,84.8,115.2$, $117.9,119.1,120.4,121.6,122.2,123.2,125.1,127.1,127.3,128.3,128.7,129.1,132.6,136.2$, 139.4, 142.5, 149.0, 160.0, 166.1, 170.6, 178.9, 192.5; HRMS (ESI-TOF) m/z: Calcd. for $\mathrm{C}_{39} \mathrm{H}_{37} \mathrm{BrN}_{2} \mathrm{NaO}_{9}[\mathrm{M}+\mathrm{Na}]^{+}:$779.1575; Found: 779.1578 .

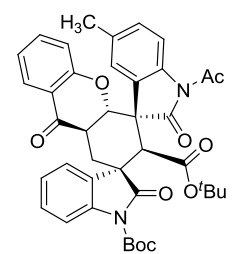

3g: White solid, m.p. $122.1-123.5{ }^{\circ} \mathrm{C} ; 42.9 \mathrm{mg}$, yield $62 \%$; $98 \%$ ee, $>20: 1 \mathrm{dr},[\alpha]_{\mathrm{D}}{ }^{20}=+142.0(c$ 0.25, $\mathrm{CH}_{2} \mathrm{Cl}_{2}$ ); The ee was determined by HPLC analysis using a Chiralpak IC column (95/15 hexane $/ i$-PrOH; flow rate: $\left.1.0 \mathrm{~mL} / \mathrm{min} ; \lambda=254 \mathrm{~nm} ; \tau_{\text {major }}=12.77 \mathrm{~min} ; \tau_{\text {minor }}=19.87 \mathrm{~min}\right) ;{ }^{1} \mathrm{H}$ $\operatorname{NMR}\left(\mathrm{CDCl}_{3}, 400 \mathrm{MHz}\right) \delta$ : 0.67 (s, 9H), 1.66 (s, 9H), 2.31 (s, 3H), 2.44-2.51 (m, 1H), 2.67-2.71 (m, 1H), $2.73(\mathrm{~s}, 3 \mathrm{H}), 3.87-3.95(\mathrm{~m}, 1 \mathrm{H}), 4.03(\mathrm{~s}, 1 \mathrm{H}), 5.90(\mathrm{~d}, J=14.4 \mathrm{~Hz}, 1 \mathrm{H}), 6.69-6.71(\mathrm{~m}$, 
1H), 6.95-6.99 (m, 1H), 7.12-7.20 (m, 2H), 7.28-7.38 (m, 3H), $7.62(\mathrm{~d}, J=1.6 \mathrm{~Hz}, 1 \mathrm{H}), 7.80-7.84$ (m, 2H), $8.15(\mathrm{~d}, J=8.4 \mathrm{~Hz}, 1 \mathrm{H}) ;{ }^{13} \mathrm{C} \mathrm{NMR}\left(\mathrm{CDCl}_{3}, 100 \mathrm{MHz}\right) \delta: 21.4,26.7,27.0,28.3,31.8$, $38.9,47.6,54.1,57.2,80.8,82.5,84.5,115.0,115.6,118.0,120.5,121.7,125.0,127.2,127.4$, $127.8,129.0,129.6,132.7,135.0,136.1,139.2,139.6,149.3,160.2,166.3,170.7,178.5,179.5$, 192.8; HRMS (ESI-TOF) m/z: Calcd. for $\mathrm{C}_{40} \mathrm{H}_{40} \mathrm{~N}_{2} \mathrm{NaO}_{9}$ [M+Na] ${ }^{+}$: 715.2626; Found: 715.2628.

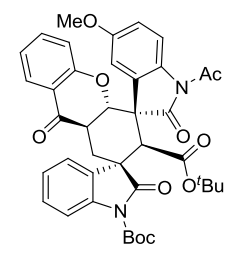

3h: White solid, m.p. $95.4-97.1{ }^{\circ} \mathrm{C} ; 46.7 \mathrm{mg}$, yield 66\%; >99\% ee, $>20: 1 \mathrm{dr},[\alpha]_{\mathrm{D}}{ }^{20}=+90.0(c 0.25$, $\mathrm{CH}_{2} \mathrm{Cl}_{2}$ ); The ee was determined by HPLC analysis using a Chiralpak IC column (95/5 hexane $/ i-\mathrm{PrOH}$; flow rate: $1.0 \mathrm{~mL} / \mathrm{min} ; \lambda=254 \mathrm{~nm} ; \tau_{\text {major }}=13.76 \mathrm{~min} ; \tau_{\text {minor }}=24.10 \mathrm{~min}$ ); ${ }^{1} \mathrm{H}$ NMR $\left(\mathrm{CDCl}_{3}, 400 \mathrm{MHz}\right) \delta: 0.71(\mathrm{~s}, 9 \mathrm{H}), 1.64(\mathrm{~s}, 9 \mathrm{H}), 2.48-2.55(\mathrm{~m}, 1 \mathrm{H}), 2.67-2.71(\mathrm{~m}, 1 \mathrm{H}), 2.74$ (s, 3H), $3.79(\mathrm{~s}, 3 \mathrm{H}), 3.89-3.98(\mathrm{~m}, 1 \mathrm{H}), 4.07(\mathrm{~s}, 1 \mathrm{H}), 5.87(\mathrm{~d}, J=14.0 \mathrm{~Hz}, 1 \mathrm{H}), 6.73(\mathrm{~d}, J=8.4$ $\mathrm{Hz}, 1 \mathrm{H})$, 6.87-6.90 (m, 1H), 6.98-7.02 (m, 1H), 7.18-7.22 (m, 1H), 7.30-7.41 (m, 3H), 7.53 (s, 1H), 7.82-7.88 (m, 2H), $8.20(\mathrm{~d}, J=8.8 \mathrm{~Hz}, 1 \mathrm{H}) ;{ }^{13} \mathrm{C} \mathrm{NMR}\left(\mathrm{CDCl}_{3}, 100 \mathrm{MHz}\right) \delta: 26.7,28.1,31.9,38.8$, 47.6, 54.4, 55.5, 56.9, 80.9, 82.5, 84.3, 111.5, 115.1, 115.4, 116.9, 117.9, 120.4, 121.6, 122.0, 124.9, 127.2, 128.9, 129.1, 132.8, 135.0, 136.0, 139.5, 149.3, 157.4, 160.1, 166.2, 170.4, 178.6, 179.3, 192.6; HRMS (ESI-TOF) m/z: Calcd. for $\mathrm{C}_{40} \mathrm{H}_{40} \mathrm{~N}_{2} \mathrm{NaO}_{10}[\mathrm{M}+\mathrm{Na}]^{+}$: 731.2575; Found: 731.2579 .

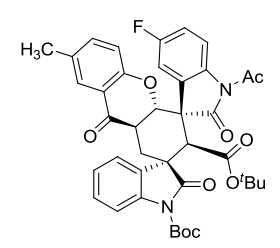

3i: White solid, m.p. $225.3-226.8{ }^{\circ} \mathrm{C} ; 51.8 \mathrm{mg}$, yield 73\%; 96\% ee, $>20: 1 \mathrm{dr},[\alpha]_{\mathrm{D}}{ }^{20}=+116.8(c$ 0.37, $\mathrm{CH}_{2} \mathrm{Cl}_{2}$ ); The ee was determined by HPLC analysis using a Chiralpak IC column $(98 / 2$ hexane $/ i-\mathrm{PrOH}$; flow rate: $1.0 \mathrm{~mL} / \mathrm{min} ; \lambda=254 \mathrm{~nm} ; \tau_{\text {major }}=16.09 \mathrm{~min} ; \tau_{\text {minor }}=39.51 \mathrm{~min}$ ); ${ }^{1} \mathrm{H}$ NMR $\left(\mathrm{CDCl}_{3}, 500 \mathrm{MHz}\right) \delta$ : $0.66(\mathrm{~s}, 9 \mathrm{H}), 1.60(\mathrm{~s}, 9 \mathrm{H}), 2.19$ (s, 3H), 2.41-2.46 (m, 1H), 2.58-2.64 (m, 1H), 2.67 (s, 3H), 3.79-3.86 (m, 1H), $3.98(\mathrm{~s}, 1 \mathrm{H}), 5.77$ (d, $J=14.0 \mathrm{~Hz}, 1 \mathrm{H}), 6.54$ (d, $J=8.5$ $\mathrm{Hz}, 1 \mathrm{H}), 6.97-7.01(\mathrm{~m}, 1 \mathrm{H}), 7.13-7.14(\mathrm{~m}, 2 \mathrm{H})$, 7.24-7.27 (m, 2H), $7.54(\mathrm{~s}, 1 \mathrm{H})$, 7.58-7.61 (m, 1H), 
$7.78(\mathrm{~d}, J=8.0 \mathrm{~Hz}, 1 \mathrm{H}), 8.20-8.23(\mathrm{~m}, 1 \mathrm{H}) ;{ }^{13} \mathrm{C} \mathrm{NMR}\left(\mathrm{CDCl}_{3}, 125 \mathrm{MHz}\right) \delta: 20.4,26.7,26.8,28.1$, $31.8,38.7,47.4,54.3,56.7,80.6,82.8,84.6,114.6\left(\mathrm{~d}, J_{C F}=26.3 \mathrm{~Hz}\right), 115.0,155.6\left(\mathrm{~d}, J_{C F}=22.5\right.$ $\mathrm{Hz}), 116.9,117.0,117.5,119.9,121.6,124.9,126.7,129.0,129.9\left(\mathrm{~d}, J_{C F}=8.8 \mathrm{~Hz}\right), 131.6,132.4$, $137.1,139.5,149.1,158.0,160.2\left(\mathrm{~d}, J_{C F}=242.5 \mathrm{~Hz}\right), 166.0,170.5,178.7,178.9,192.6 ;{ }^{19} \mathrm{~F}$ NMR $\left(\mathrm{CDCl}_{3}, 470 \mathrm{MHz}\right) \delta$ : -115.69 ; HRMS (ESI-TOF) m/z: Calcd. for $\mathrm{C}_{40} \mathrm{H}_{39} \mathrm{FN}_{2} \mathrm{NaO}_{9}[\mathrm{M}+\mathrm{Na}]^{+}$: 733.2532; Found: 733.2537.

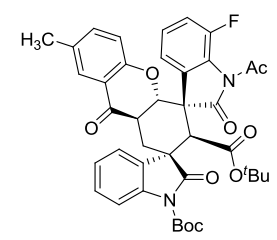

3j: White solid, m.p. $207.0-208.9{ }^{\circ} \mathrm{C} ; 50.4 \mathrm{mg}$, yield 71\%; $97 \%$ ee, $>20: 1 \mathrm{dr},[\alpha]_{\mathrm{D}}{ }^{20}=+114.1(c$ $0.27, \mathrm{CH}_{2} \mathrm{Cl}_{2}$ ); The ee was determined by HPLC analysis using a Chiralpak IC column (95/5 hexane/i-PrOH; flow rate: $1.0 \mathrm{~mL} / \mathrm{min} ; \lambda=254 \mathrm{~nm} ; \tau_{\text {major }}=21.84 \mathrm{~min} ; \tau_{\text {minor }}=44.88 \mathrm{~min}$ ); ${ }^{1} \mathrm{H}$ NMR $\left(\mathrm{CDCl}_{3}, 500 \mathrm{MHz}\right) \delta: 0.66(\mathrm{~s}, 9 \mathrm{H}), 1.60$ (s, 9H), 2.19 (s, 3H), 2.38-2.43 (m, 1H), 2.59-2.64 (m, 1H), $2.68(\mathrm{~s}, 3 \mathrm{H}), 3.74-3.81(\mathrm{~m}, 1 \mathrm{H}), 4.00(\mathrm{~s}, 1 \mathrm{H}), 5.75(\mathrm{~d}, J=14.0 \mathrm{~Hz}, 1 \mathrm{H}), 6.54(\mathrm{~d}, J=7.5$ $\mathrm{Hz}, 1 \mathrm{H}), 7.05-7.09(\mathrm{~m}, 1 \mathrm{H}), 7.11-7.14(\mathrm{~m}, 3 \mathrm{H}), 7.23-7.27$ (m, 2H), $7.53(\mathrm{~s}, 1 \mathrm{H}), 7.61(\mathrm{~d}, J=7.5$ $\mathrm{Hz}, 1 \mathrm{H}), 7.72(\mathrm{~d}, J=8.0 \mathrm{~Hz}, 1 \mathrm{H}) ;{ }^{13} \mathrm{C} \mathrm{NMR}\left(\mathrm{CDCl}_{3}, 125 \mathrm{MHz}\right) \delta: 20.4,26.1,26.8,28.2,31.7$, $38.7,47.5,55.2,56.2,80.8,82.8,84.6,115.1,117.6,119.9,121.5,122.6,124.9,126.7,128.9$, 131.6, 132.7, 137.1, 139.4, 149.0, $149.4\left(\mathrm{~d}, J_{C F}=242.5 \mathrm{~Hz}\right), 158.0,166.2,168.1,178.5,178.8$, 192.6; ${ }^{19} \mathrm{~F} \quad \mathrm{NMR}\left(\mathrm{CDCl}_{3}, 470 \mathrm{MHz}\right) \delta:-112.47$; HRMS (ESI-TOF) $\mathrm{m} / \mathrm{z}$ : Calcd. for $\mathrm{C}_{40} \mathrm{H}_{39} \mathrm{FN}_{2} \mathrm{NaO}_{9}[\mathrm{M}+\mathrm{Na}]^{+}:$733.2532; Found: 733.2538.

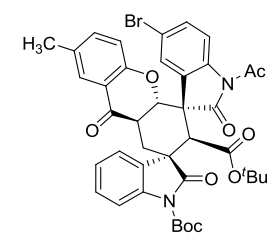

3k: White solid, m.p. $154.6-156.5{ }^{\circ} \mathrm{C} ; 55.4 \mathrm{mg}$, yield $72 \%$; $>25 \%$ ee, $>20: 1 \mathrm{dr},[\alpha]_{\mathrm{D}}{ }^{20}=+101.5(c$ 0.34, $\mathrm{CH}_{2} \mathrm{Cl}_{2}$ ); The ee was determined by HPLC analysis using a Chiralpak IC column (98/2 hexane $/ i$-PrOH; flow rate: $1.0 \mathrm{~mL} / \mathrm{min} ; \lambda=254 \mathrm{~nm} ; \tau_{\text {major }}=15.21 \mathrm{~min} ; \tau_{\text {minor }}=37.02 \mathrm{~min}$ ); ${ }^{1} \mathrm{H}$ NMR $\left(\mathrm{CDCl}_{3}, 500 \mathrm{MHz}\right) \delta$ : $0.66(\mathrm{~s}, 9 \mathrm{H}), 1.60$ (s, 9H), 2.20 (s, 3H), 2.41-2.46 (m, 1H), 2.58-2.63 (m, 1H), $2.67(\mathrm{~s}, 3 \mathrm{H}), 3.78-3.85(\mathrm{~m}, 1 \mathrm{H}), 3.96(\mathrm{~s}, 1 \mathrm{H}), 5.82(\mathrm{~d}, J=14.0 \mathrm{~Hz}, 1 \mathrm{H}), 6.55(\mathrm{~d}, J=8.0$ 
$\mathrm{Hz}, 1 \mathrm{H}), 7.12-7.15(\mathrm{~m}, 2 \mathrm{H}), 7.25-7.27(\mathrm{~m}, 2 \mathrm{H}), 7.40-7.42(\mathrm{~m}, 1 \mathrm{H}), 7.55(\mathrm{~s}, 1 \mathrm{H}), 7.84(\mathrm{~d}, J=7.5$ $\mathrm{Hz}, 1 \mathrm{H}), 7.94(\mathrm{~d}, J=2.0 \mathrm{~Hz}, 1 \mathrm{H}), 8.12(\mathrm{~d}, J=8.5 \mathrm{~Hz}, 1 \mathrm{H}) ;{ }^{13} \mathrm{C} \mathrm{NMR}\left(\mathrm{CDCl}_{3}, 125 \mathrm{MHz}\right) \delta: 20.4$, 26.6, 26.8, 28.1, 31.8, 38.7, 47.4, 54.1, 57.1, 80.5, 83.1, 84.6, 115.0, 117.3, 117.6, 118.4, 120.0, $121.7,125.0,126.7,129.1,130.1,131.6,132.1,132.2,137.1,139.6,140.5,149.3,158.0,166.0$, 170.5, 178.4, 192.6; HRMS (ESI-TOF) m/z: Calcd. for $\mathrm{C}_{40} \mathrm{H}_{39} \mathrm{BrN}_{2} \mathrm{NaO}_{9}[\mathrm{M}+\mathrm{Na}]^{+}$: 793.1731; Found: 793.1733 .

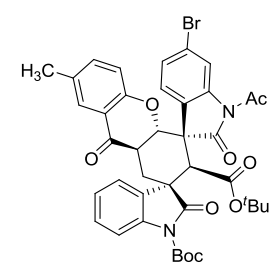

31: White solid, m.p. $157.3-158.9{ }^{\circ} \mathrm{C} ; 53.9 \mathrm{mg}$, yield 70\%; $97 \%$ ee, $>20: 1 \mathrm{dr},[\alpha]_{\mathrm{D}}{ }^{20}=+102.6(c$ 0.27, $\mathrm{CH}_{2} \mathrm{Cl}_{2}$ ); The ee was determined by HPLC analysis using a Chiralpak IC column (98/2 hexane $/ i$-PrOH; flow rate: $\left.1.0 \mathrm{~mL} / \mathrm{min} ; \lambda=254 \mathrm{~nm} ; \tau_{\text {major }}=15.77 \mathrm{~min} ; \tau_{\text {minor }}=42.28 \mathrm{~min}\right) ;{ }^{1} \mathrm{H}$ $\operatorname{NMR}\left(\mathrm{CDCl}_{3}, 500 \mathrm{MHz}\right) \delta$ : 0.77 (s, 9H), $1.72(\mathrm{~s}, 9 \mathrm{H}), 2.31$ (s, 3H), 2.51-2.56 (m, 1H), 2.69-2.74 (m, 1H), 2.79 (s, 3H), 3.88-3.95 (m, 1H), $4.08(\mathrm{~s}, 1 \mathrm{H}), 5.83(\mathrm{~d}, J=14.0 \mathrm{~Hz}, 1 \mathrm{H}), 6.66(\mathrm{~d}, J=8.5$ $\mathrm{Hz}, 1 \mathrm{H})$, 7.23-7.26 (m, 2H), 7.34-7.38 (m, 2H), 7.40-7.42 (m, 1H), 7.65 (s, 1H), 7.81-7.84 (m, 2H), $8.56(\mathrm{~d}, J=2.0 \mathrm{~Hz}, 1 \mathrm{H}) ;{ }^{13} \mathrm{C} \mathrm{NMR}\left(\mathrm{CDCl}_{3}, 125 \mathrm{MHz}\right) \delta: 20.4,26.7,26.9,28.1,31.9,38.7,47.4$, 53.9, 56.5, 80.6, 82.8, 84.7, 115.1, 117.6, 118.9, 119.9, 121.5, 123.0, 125.0, 126.7, 127.1, 128.2, $128.5,128.9,131.6,132.6,137.1,139.3,142.4,148.9,158.0,166.0,170.5,178.8,178.9,192.6$; HRMS (ESI-TOF) m/z: Calcd. for $\mathrm{C}_{40} \mathrm{H}_{39} \mathrm{BrN}_{2} \mathrm{NaO}_{9}[\mathrm{M}+\mathrm{Na}]^{+}$: 793.1731 ; Found: 793.1736 .

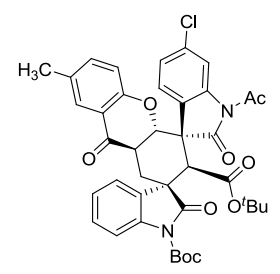

3m: White solid, m.p. $146.3-148.0{ }^{\circ} \mathrm{C} ; 51.5 \mathrm{mg}$, yield 71\%; 96\% ee, $>20: 1 \mathrm{dr},[\alpha]_{\mathrm{D}}{ }^{20}=+102.2(c$ 0.18, $\mathrm{CH}_{2} \mathrm{Cl}_{2}$ ); The ee was determined by HPLC analysis using a Chiralpak IC column (95/5 hexane $/ i$-PrOH; flow rate: $\left.1.0 \mathrm{~mL} / \mathrm{min} ; \lambda=254 \mathrm{~nm} ; \tau_{\text {major }}=9.35 \mathrm{~min} ; \tau_{\text {minor }}=21.66 \mathrm{~min}\right) ;{ }^{1} \mathrm{H}$ NMR (CDCl $3,500 \mathrm{MHz}) \delta$ : $0.65(\mathrm{~s}, 9 \mathrm{H}), 1.60(\mathrm{~s}, 9 \mathrm{H}), 2.19$ (s, 3H), 2.40-2.45 (m, 1H), 2.58-2.63 (m, 1H), $2.68(\mathrm{~s}, 3 \mathrm{H}), 3.77-3.84(\mathrm{~m}, 1 \mathrm{H}), 3.97(\mathrm{~s}, 1 \mathrm{H}), 5.72(\mathrm{~d}, J=14.0 \mathrm{~Hz}, 1 \mathrm{H}), 6.55(\mathrm{~d}, J=8.5$ $\mathrm{Hz}, 1 \mathrm{H}), 7.11-7.14(\mathrm{~m}, 3 \mathrm{H}), 7.23-7.26(\mathrm{~m}, 2 \mathrm{H}), 7.54(\mathrm{~s}, 1 \mathrm{H}), 7.72(\mathrm{~d}, J=8.0 \mathrm{~Hz}, 1 \mathrm{H}), 7.76(\mathrm{~d}, J=$ 
8.5 Hz, 1H), 8.29 (s, 1H); ${ }^{13} \mathrm{C} \mathrm{NMR}\left(\mathrm{CDCl}_{3}, 125 \mathrm{MHz}\right) \delta: 20.4,26.7,26.9,28.1,31.9,38.7,47.4$, 53.9, 56.6, 80.7, 82.8, 84.7, 115.1, 116.2, 117.6, 119.9, 121.5, 125.0, 125.6, 126.5, 126.7, 127.8, 128.9, 131.6, 132.6, 135.0, 137.1, 139.3, 142.2, 148.9, 158.0, 166.0, 170.5, 178.8, 179.0, 192.6; HRMS (ESI-TOF) m/z: Calcd. for $\mathrm{C}_{40} \mathrm{H}_{39} \mathrm{ClN}_{2} \mathrm{NaO}_{9}[\mathrm{M}+\mathrm{Na}]^{+}$: 749.2236; Found: 749.2240.

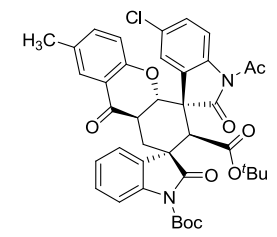

3n: White solid, m.p. $216.3-217.8{ }^{\circ} \mathrm{C}$; $55.9 \mathrm{mg}$, yield 77\%; $>99 \%$ ee, $>20: 1 \mathrm{dr},[\alpha]_{\mathrm{D}}{ }^{20}=+119.6(c$ 0.24, $\mathrm{CH}_{2} \mathrm{Cl}_{2}$ ); The ee was determined by HPLC analysis using a Chiralpak IC column (98/2 hexane $/ i$-PrOH; flow rate: $\left.1.0 \mathrm{~mL} / \mathrm{min} ; \lambda=254 \mathrm{~nm} ; \tau_{\text {major }}=14.72 \mathrm{~min} ; \tau_{\text {minor }}=36.92 \mathrm{~min}\right) ;{ }^{1} \mathrm{H}$ $\operatorname{NMR}\left(\mathrm{CDCl}_{3}, 500 \mathrm{MHz}\right) \delta: 0.66(\mathrm{~s}, 9 \mathrm{H}), 1.60(\mathrm{~s}, 9 \mathrm{H}), 2.20(\mathrm{~s}, 3 \mathrm{H}), 2.41-2.46(\mathrm{~m}, 1 \mathrm{H}), 2.58-2.64$ (m, 1H), 2.67 (s, 3H), 3.79-3.85 (m, 1H), $3.96(\mathrm{~s}, 1 \mathrm{H}), 5.81(\mathrm{~d}, J=14.0 \mathrm{~Hz}, 1 \mathrm{H}), 6.55(\mathrm{~d}, J=8.5$ $\mathrm{Hz}, 1 \mathrm{H}), 7.12-7.15(\mathrm{~m}, 2 \mathrm{H}), 7.24-7.27$ (m, 3H), 7.55 (s, 1H), 7.80-7.83 (m, 2H), 8.18 (d, $J=8.5$ $\mathrm{Hz}, 1 \mathrm{H}) ;{ }^{13} \mathrm{C} \mathrm{NMR}\left(\mathrm{CDCl}_{3}, 125 \mathrm{MHz}\right) \delta: 20.4,26.6,26.8,28.1,31.8,38.7,47.4,54.2,57.0$, 80.5, 83.0, 84.6, 114.9, 116.9, 117.6, 120.0, 121.7, 125.0, 126.7, 127.3, 129.0, 129.2, 129.7, 130.7, 131.6, 132.3, 137.1, 140.0, 149.3, 158.0, 166.0, 170.5, 178.4, 178.5, 192.6; HRMS (ESI-TOF) m/z: Calcd. for $\mathrm{C}_{40} \mathrm{H}_{39} \mathrm{ClN}_{2} \mathrm{NaO}_{9}[\mathrm{M}+\mathrm{Na}]^{+}$: 749.2236; Found: 749.2241 .

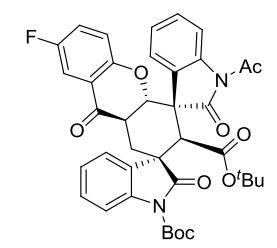

3o: White solid, m.p. $211.2-212.5{ }^{\circ} \mathrm{C}$; $59.1 \mathrm{mg}$, yield $85 \%$; $94 \%$ ee, $>20: 1 \mathrm{dr},[\alpha]_{\mathrm{D}}{ }^{20}=+143.8(c$ 0.29, $\mathrm{CH}_{2} \mathrm{Cl}_{2}$ ); The ee was determined by HPLC analysis using a Chiralpak IA column $(98 / 2$ hexane $/ i$-PrOH; flow rate: $\left.1.0 \mathrm{~mL} / \mathrm{min} ; \lambda=254 \mathrm{~nm} ; \tau_{\text {major }}=30.30 \mathrm{~min} ; \tau_{\text {minor }}=18.86 \mathrm{~min}\right) ;{ }^{1} \mathrm{H}$ NMR $\left(\mathrm{CDCl}_{3}, 400 \mathrm{MHz}\right) \delta: 0.66(\mathrm{~s}, 9 \mathrm{H}), 1.66(\mathrm{~s}, 9 \mathrm{H}), 2.45-2.53(\mathrm{~m}, 1 \mathrm{H}), 2.65-2.70(\mathrm{~m}, 1 \mathrm{H}), 2.74$ (s, 3H), 3.86-3.94 (m, 1H), $4.04(\mathrm{~s}, 1 \mathrm{H}), 5.88(\mathrm{~d}, J=14.0 \mathrm{~Hz}, 1 \mathrm{H}), 6.65-6.69(\mathrm{~m}, 1 \mathrm{H})$, 7.05-7.10 (m, 1H), 7.16-7.22 (m, 2H), 7.28-7.37 (m, 3H), 7.44-7.47 (m, 1H), $7.77(\mathrm{~d}, J=8.0 \mathrm{~Hz}, 1 \mathrm{H})$, 7.83-7.86 (m, 1H), $8.26(\mathrm{~d}, J=8.0 \mathrm{~Hz}, 1 \mathrm{H}) ;{ }^{13} \mathrm{C} \mathrm{NMR}\left(\mathrm{CDCl}_{3}, 100 \mathrm{MHz}\right) \delta: 26.8,27.0,28.2,31.8$, $38.9,47.6,54.0,57.0,81.1,82.7,84.8,112.2\left(\mathrm{~d}, J_{C F}=23.3 \mathrm{~Hz}\right), 115.2,115.8,119.7\left(\mathrm{~d}, J_{C F}=7.5\right.$ 
$\mathrm{Hz}), 120.9\left(\mathrm{~d}, J_{C F}=6.7 \mathrm{~Hz}\right), 121.6,123.6\left(\mathrm{~d}, J_{C F}=24.5 \mathrm{~Hz}\right), 125.0,125.8,126.8,127.9,129.0$, $129.4,132.6,139.4,141.5,149.0,156.4,158.8\left(\mathrm{~d}, J_{C F}=238.2 \mathrm{~Hz}\right), 170.8,178.8,179.4,192.0 ;{ }^{19} \mathrm{~F}$ NMR $\left(\mathrm{CDCl}_{3}, 376 \mathrm{MHz}\right) \delta$ : -120.48; HRMS (ESI-TOF) m/z: Calcd. for $\mathrm{C}_{39} \mathrm{H}_{37} \mathrm{FN}_{2} \mathrm{NaO}_{9}[\mathrm{M}+\mathrm{Na}]^{+}:$ 719.2375; Found: 719.2379 .

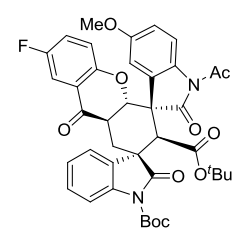

3p: White solid, m.p. $138.5-140.1{ }^{\circ} \mathrm{C} ; 45.0 \mathrm{mg}$, yield $62 \%$; $97 \%$ ee, $>20: 1 \mathrm{dr},[\alpha]_{\mathrm{D}}{ }^{20}=+127.0(c$ $\left.0.23, \mathrm{CH}_{2} \mathrm{Cl}_{2}\right)$; The ee was determined by HPLC analysis using a Chiralpak IC column $(95 / 5$ hexane $/ i$-PrOH; flow rate: $\left.1.0 \mathrm{~mL} / \mathrm{min} ; \lambda=254 \mathrm{~nm} ; \tau_{\text {major }}=14.59 \mathrm{~min} ; \tau_{\text {minor }}=25.87 \mathrm{~min}\right) ;{ }^{1} \mathrm{H}$ $\operatorname{NMR}\left(\mathrm{CDCl}_{3}, 500 \mathrm{MHz}\right) \delta: 0.64(\mathrm{~s}, 9 \mathrm{H}), 1.57$ (s, 9H), 2.42-2.47 (m, 1H), 2.58-2.62 (m, 1H), 2.66 (s, 3H), $3.72(\mathrm{~s}, 3 \mathrm{H}), 3.79-3.89(\mathrm{~m}, 1 \mathrm{H}), 3.98(\mathrm{~s}, 1 \mathrm{H}), 5.80(\mathrm{~d}, J=14.5 \mathrm{~Hz}, 1 \mathrm{H}), 6.64-6.66(\mathrm{~m}, 1 \mathrm{H})$, 6.81-6.83 (m, 1H), 7.02-7.06 (m, 1H), 7.12-7.15 (m, 1H), 7.24-7.27 (m, 2H), 7.40-7.42 (m, 1H), $7.44(\mathrm{~d}, J=2.5 \mathrm{~Hz}, 1 \mathrm{H}), 7.79(\mathrm{~d}, J=8.0 \mathrm{~Hz}, 1 \mathrm{H}), 8.12(\mathrm{~d}, J=9.0 \mathrm{~Hz}, 1 \mathrm{H}) ;{ }^{13} \mathrm{C} \mathrm{NMR}\left(\mathrm{CDCl}_{3}, 125\right.$ MHz) $\delta: 26.7,26.8,28.1,31.8,38.8,47.5,54.2,55.5,57.0,81.1,82.6,84.4,111.5,112.1\left(\mathrm{~d}, J_{C F}=\right.$ $22.5 \mathrm{~Hz}), 115.1,115.4,116.8,119.6,119.7,121.5,123.5\left(\mathrm{~d}, J_{C F}=25.0 \mathrm{~Hz}\right), 125.0,128.9,129.0$, $132.6,134.9,139.5,149.2,156.3,156.6,157.5\left(\mathrm{~d}, J_{C F}=242.5 \mathrm{~Hz}\right), 166.1,170.3,178.6,179.2$, 192.0; ${ }^{19} \mathrm{~F} \quad \mathrm{NMR}\left(\mathrm{CDCl}_{3}, 470 \mathrm{MHz}\right) \quad \delta:-120.49 ; \quad \mathrm{HRMS} \quad$ (ESI-TOF) $\mathrm{m} / \mathrm{z}$ : Calcd. for $\mathrm{C}_{40} \mathrm{H}_{39} \mathrm{FN}_{2} \mathrm{NaO}_{10}[\mathrm{M}+\mathrm{Na}]^{+}:$749.2481; Found: 749.2485 .

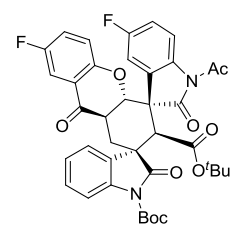

3q: White solid, m.p. $201.4-203.2{ }^{\circ} \mathrm{C}$; $64.3 \mathrm{mg}$, yield 90\%; 98\% ee, $>20: 1 \mathrm{dr},[\alpha]_{\mathrm{D}}{ }^{20}=+127.7(c$ 0.30, $\left.\mathrm{CH}_{2} \mathrm{Cl}_{2}\right)$; The ee was determined by HPLC analysis using a Chiralpak IC column (95/5 hexane $/ i$-PrOH; flow rate: $\left.1.0 \mathrm{~mL} / \mathrm{min} ; \lambda=254 \mathrm{~nm} ; \tau_{\text {major }}=16.07 \mathrm{~min} ; \tau_{\text {minor }}=33.64 \mathrm{~min}\right) ;{ }^{1} \mathrm{H}$ $\operatorname{NMR}\left(\mathrm{CDCl}_{3}, 400 \mathrm{MHz}\right) \delta: 0.71(\mathrm{~s}, 9 \mathrm{H}), 1.65$ (s, 9H), 2.46-2.53 (m, 1H), 2.62-2.68 (m, 1H), 2.73 (s, 3H), 3.85-3.93 (m, 1H), $4.02(\mathrm{~s}, 1 \mathrm{H}), 5.87(\mathrm{~d}, J=14.0 \mathrm{~Hz}, 1 \mathrm{H}), 6.67-6.70(\mathrm{~m}, 1 \mathrm{H}), 7.02-7.12$ (m, 2H), 7.17-7.21 (m, 1H), 7.29-7.33 (m, 2H), 7.45-7.47 (m, 1H), 7.62-7.65 (m, 1H), 7.82-7.84 
(m, 1H), 8.25-8.29 (m, 1H); ${ }^{13} \mathrm{C} \mathrm{NMR}\left(\mathrm{CDCl}_{3}, 100 \mathrm{MHz}\right) \delta: 26.8,27.0,28.2,31.7,38.8,47.6$, $54.2,57.0,81.0,83.0,84.8,112.3\left(\mathrm{~d}, J_{C F}=23.0 \mathrm{~Hz}\right), 114.7\left(\mathrm{~d}, J_{C F}=26.1 \mathrm{~Hz}\right), 115.8\left(\mathrm{~d}, J_{C F}=23.0\right.$ $\mathrm{Hz}), 116.0,119.6,119.7,121.0,121.7,123.6\left(\mathrm{~d}, J_{C F}=25.1 \mathrm{~Hz}\right), 125.1,129.2,129.8\left(\mathrm{~d}, J_{C F}=9.0\right.$ $\mathrm{Hz}), 132.3,139.6,149.2,156.2,157.2\left(\mathrm{~d}, J_{C F}=242.2 \mathrm{~Hz}\right), 160.4\left(\mathrm{~d}, J_{C F}=243.5 \mathrm{~Hz}\right), 166.0,170.5$, 178.8, 178.9, 191.8; ${ }^{19} \mathrm{~F}$ NMR $\left(\mathrm{CDCl}_{3}, 376 \mathrm{MHz}\right) \delta$ : -115.53, -120.22; HRMS (ESI-TOF) m/z: Calcd. for $\mathrm{C}_{39} \mathrm{H}_{36} \mathrm{~F}_{2} \mathrm{~N}_{2} \mathrm{NaO}_{9}[\mathrm{M}+\mathrm{Na}]^{+}$: 737.2281; Found: 737.2287.

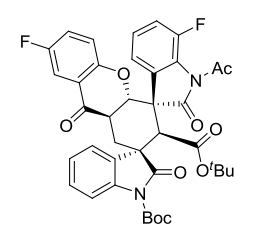

3r: White solid, m.p. $111.2-113.0{ }^{\circ} \mathrm{C} ; 62.1 \mathrm{mg}$, yield $87 \%$; >99\% ee, $>20: 1 \mathrm{dr},[\alpha]_{\mathrm{D}}{ }^{20}=+159.5(c$ 0.20, $\mathrm{CH}_{2} \mathrm{Cl}_{2}$ ); The ee was determined by HPLC analysis using a Chiralpak IC column $(95 / 5$ hexane/i-PrOH; flow rate: $\left.1.0 \mathrm{~mL} / \mathrm{min} ; \lambda=254 \mathrm{~nm} ; \tau_{\text {major }}=22.72 \mathrm{~min} ; \tau_{\text {minor }}=32.36 \mathrm{~min}\right) ;{ }^{1} \mathrm{H}$ $\operatorname{NMR}\left(\mathrm{CDCl}_{3}, 500 \mathrm{MHz}\right) \delta: 0.66(\mathrm{~s}, 9 \mathrm{H}), 1.61(\mathrm{~s}, 9 \mathrm{H}), 2.39-2.44(\mathrm{~m}, 1 \mathrm{H}), 2.59-2.64(\mathrm{~m}, 1 \mathrm{H}), 2.69$ (s, 3H), 3.76-3.82 (m, 1H), $3.99(\mathrm{~s}, 1 \mathrm{H}), 5.81(\mathrm{~d}, J=14.5 \mathrm{~Hz}, 1 \mathrm{H}), 6.62-6.64(\mathrm{~m}, 1 \mathrm{H}), 7.02-7.10$ (m, 2H), 7.12-7.16 (m, 2H), 7.24-7.27 (m, 2H), 7.39-7.41 (m, 1H), $7.59(\mathrm{~d}, J=8.0 \mathrm{~Hz}, 1 \mathrm{H}), 7.72$ $(\mathrm{d}, J=8.0 \mathrm{~Hz}, 1 \mathrm{H}) ;{ }^{13} \mathrm{C} \mathrm{NMR}\left(\mathrm{CDCl}_{3}, 125 \mathrm{MHz}\right) \delta: 26.1,26.8,28.1,31.5,38.7,47.5,55.0,56.3$, $81.0,82.9,84.7,112.1\left(\mathrm{~d}, J_{C F}=23.8 \mathrm{~Hz}\right), 115.1,117.8\left(\mathrm{~d}, J_{C F}=20.0 \mathrm{~Hz}\right), 119.5,121.5,123.6(\mathrm{~d}$, $\left.J_{C F}=25.0 \mathrm{~Hz}\right), 125.0,126.8,129.0,132.4,139.3,148.9,149.3\left(\mathrm{~d}, J_{C F}=258.8 \mathrm{~Hz}\right), 156.1,157.5$ $\left(\mathrm{d}, J_{C F}=241.3 \mathrm{~Hz}\right), 166.0,168.0,178.3,178.8,191.7 ;{ }^{19} \mathrm{~F} \mathrm{NMR}\left(\mathrm{CDCl}_{3}, 470 \mathrm{MHz}\right) \delta:-112.38$, -120.29; HRMS (ESI-TOF) m/z: Calcd. for $\mathrm{C}_{39} \mathrm{H}_{36} \mathrm{~F}_{2} \mathrm{~N}_{2} \mathrm{NaO}_{9}[\mathrm{M}+\mathrm{Na}]^{+}$: 737.2281; Found: 737.2285 .

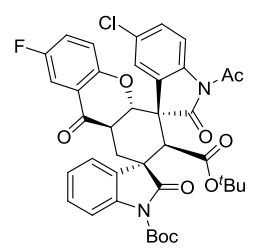

3s: White solid, m.p. $116.5-122.7{ }^{\circ} \mathrm{C} ; 64.2 \mathrm{mg}$, yield $88 \%$; >99\% ee, $>20: 1 \mathrm{dr},[\alpha]_{\mathrm{D}}{ }^{20}=+132.0(c$ 0.30, $\mathrm{CH}_{2} \mathrm{Cl}_{2}$ ); The ee was determined by HPLC analysis using a Chiralpak IC column (98/2 hexane $/ i$-PrOH; flow rate: $1.0 \mathrm{~mL} / \mathrm{min} ; \lambda=254 \mathrm{~nm} ; \tau_{\text {major }}=13.28 \mathrm{~min} ; \tau_{\text {minor }}=24.60 \mathrm{~min}$ ); ${ }^{1} \mathrm{H}$ NMR $\left(\mathrm{CDCl}_{3}, 400 \mathrm{MHz}\right) \delta: 0.71(\mathrm{~s}, 9 \mathrm{H}), 1.66(\mathrm{~s}, 9 \mathrm{H}), 2.46-2.53(\mathrm{~m}, 1 \mathrm{H}), 2.63-2.69(\mathrm{~m}, 1 \mathrm{H}), 2.73$ 
(s, 3H), 3.85-3.93 (m, 1H), $4.00(\mathrm{~s}, 1 \mathrm{H}), 5.91(\mathrm{~d}, J=14.0 \mathrm{~Hz}, 1 \mathrm{H}), 6.68-6.71(\mathrm{~m}, 1 \mathrm{H}), 7.08-7.13$ (m, 1H), 7.17-7.21 (m, 1H), 7.30-7.34 (m, 3H), 7.45-7.48 (m, 1H), $7.85(\mathrm{~d}, J=2.4 \mathrm{~Hz}, 1 \mathrm{H})$, 7.86-7.89 (m, 1H), $8.23(\mathrm{~d}, J=8.8 \mathrm{~Hz}, 1 \mathrm{H}) ;{ }^{13} \mathrm{C} \mathrm{NMR}\left(\mathrm{CDCl}_{3}, 100 \mathrm{MHz}\right) \delta: 26.7,26.9,28.2,31.7$, $38.8,47.6,54.1,57.3,80.9,83.2,84.8,112.3\left(\mathrm{~d}, J_{C F}=23.1 \mathrm{~Hz}\right), 115.1,117.1,121.0,121.8,123.6$ $\left(\mathrm{d}, J_{C F}=25.1 \mathrm{~Hz}\right), 125.1,127.4,129.3,129.4,129.6,130.9,132.1,139.6,140.1,149.3,156.2$, $157.6\left(\mathrm{~d}, J_{C F}=242.1 \mathrm{~Hz}\right), 166.0,170.6,178.5,178.6,191.8 ;{ }^{19} \mathrm{~F}$ NMR $\left(\mathrm{CDCl}_{3}, 376 \mathrm{MHz}\right) \delta$ : -120.19; HRMS (ESI-TOF) m/z: Calcd. for $\mathrm{C}_{39} \mathrm{H}_{36} \mathrm{ClFN}_{2} \mathrm{NaO}_{9}[\mathrm{M}+\mathrm{Na}]^{+}$: 753.1986; Found: 753.1991

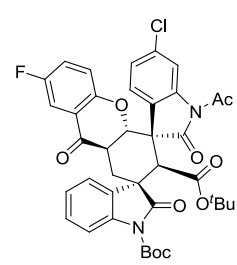

3t: White solid, m.p. $121.1-122.6{ }^{\circ} \mathrm{C} ; 58.4 \mathrm{mg}$, yield $80 \%$; $98 \%$ ee, $>20: 1 \mathrm{dr},[\alpha]_{\mathrm{D}}{ }^{20}=+100.8(c$ $\left.0.13, \mathrm{CH}_{2} \mathrm{Cl}_{2}\right)$; The ee was determined by HPLC analysis using a Chiralpak IC column $(95 / 5$ hexane/i-PrOH; flow rate: $\left.1.0 \mathrm{~mL} / \mathrm{min} ; \lambda=254 \mathrm{~nm} ; \tau_{\text {major }}=13.91 \mathrm{~min} ; \tau_{\text {minor }}=32.35 \mathrm{~min}\right) ;{ }^{1} \mathrm{H}$ NMR $\left(\mathrm{CDCl}_{3}, 400 \mathrm{MHz}\right) \delta: 0.71(\mathrm{~s}, 9 \mathrm{H}), 1.66(\mathrm{~s}, 9 \mathrm{H}), 2.45-2.51(\mathrm{~m}, 1 \mathrm{H}), 2.62-2.70(\mathrm{~m}, 1 \mathrm{H}), 2.73$ (s, 3H), 3.83-3.91 (m, 1H), $4.02(\mathrm{~s}, 1 \mathrm{H}), 5.83(\mathrm{~d}, J=14.4 \mathrm{~Hz}, 1 \mathrm{H}), 6.68-6.71(\mathrm{~m}, 1 \mathrm{H}), 7.08-7.13$ (m, 1H), 7.17-7.21 (m, 2H), 7.28-7.32 (m, 2H), 7.44-7.47 (m, 1H), 7.76-7.78 (m, 1H), $7.80(\mathrm{~d}, J=$ $8.0 \mathrm{~Hz}, 1 \mathrm{H}), 8.34(\mathrm{~d}, J=2.0 \mathrm{~Hz}, 1 \mathrm{H}) ;{ }^{13} \mathrm{C} \mathrm{NMR}\left(\mathrm{CDCl}_{3}, 100 \mathrm{MHz}\right) \delta: 26.8,26.9,28.2,31.8,38.8$, 47.6, 53.8, 56.8, 81.0, 83.0, 84.9, $112.3\left(\mathrm{~d}, J_{C F}=23.1 \mathrm{~Hz}\right), 115.2,116.4,119.7\left(\mathrm{~d}, J_{C F}=8.2 \mathrm{~Hz}\right)$, $120.9\left(\mathrm{~d}, J_{C F}=7.3 \mathrm{~Hz}\right), 121.6,123.6\left(\mathrm{~d}, J_{C F}=25.3 \mathrm{~Hz}\right), 125.1,125.7,126.4,127.9,129.1,132.5$, $135.2,139.4,142.3,149.0,156.2,157.6\left(\mathrm{~d}, J_{C F}=241.5 \mathrm{~Hz}\right), 166.0,170.6,178.9,179.0,191.8 ;{ }^{19} \mathrm{~F}$ NMR $\left(\mathrm{CDCl}_{3}, 376 \mathrm{MHz}\right) \delta$ : -120.18; HRMS (ESI-TOF) m/z: Calcd. for $\mathrm{C}_{39} \mathrm{H}_{36} \mathrm{ClFN}_{2} \mathrm{NaO}_{9}$ $[\mathrm{M}+\mathrm{Na}]^{+}:$753.1986; Found: 753.1992.

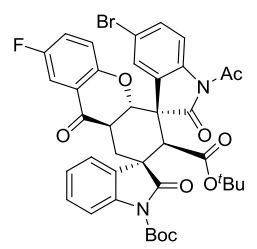

3u: White solid, m.p. $101.1-102.3{ }^{\circ} \mathrm{C} ; 70.4 \mathrm{mg}$, yield $91 \%$; $98 \%$ ee, $10: 1 \mathrm{dr},[\alpha]_{\mathrm{D}}^{20}=+99.1(c 0.45$, $\mathrm{CH}_{2} \mathrm{Cl}_{2}$ ); The ee was determined by HPLC analysis using a Chiralpak IA column (98/2 
hexane $/ i-\mathrm{PrOH}$; flow rate: $1.0 \mathrm{~mL} / \mathrm{min} ; \lambda=254 \mathrm{~nm} ; \tau_{\text {major }}=13.22 \mathrm{~min} ; \tau_{\text {minor }}=23.73 \mathrm{~min}$ ); ${ }^{1} \mathrm{H}$ NMR $\left(\mathrm{CDCl}_{3}, 400 \mathrm{MHz}\right) \delta$ : 0.71 (s, 9H), $1.66(\mathrm{~s}, 9 \mathrm{H}), 2.46-2.53(\mathrm{~m}, 1 \mathrm{H}), 2.62-2.69(\mathrm{~m}, 1 \mathrm{H}), 2.72$ (s, 3H), 3.85-3.93 (m, 1H), $4.00(\mathrm{~s}, 1 \mathrm{H}), 5.91(\mathrm{~d}, J=14.0 \mathrm{~Hz}, 1 \mathrm{H}), 6.68-6.72(\mathrm{~m}, 1 \mathrm{H}), 7.08-7.13$ (m, 1H), 7.17-7.21 (m, 1H), 7.30-7.34 (m, 2H), 7.45-7.49 (m, 2H), 7.88-7.90 (m, 1H), 7.98 (s, 1H), $8.17(\mathrm{~d}, J=8.8 \mathrm{~Hz}, 1 \mathrm{H}) ;{ }^{13} \mathrm{C} \mathrm{NMR}\left(\mathrm{CDCl}_{3}, 100 \mathrm{MHz}\right) \delta: 26.7 ., 26.9,28.2,31.7,38.8$, 47.6, 54.1, 57.3, 80.9, 83.3, 84.8, $112.3\left(\mathrm{~d}, J_{C F}=23.3 \mathrm{~Hz}\right), 115.1,117.5,118.6,119.7\left(\mathrm{~d}, J_{C F}=8.1 \mathrm{~Hz}\right), 121.0$ $\left(\mathrm{d}, J_{C F}=7.5 \mathrm{~Hz}\right), 121.1,121.8,123.6\left(\mathrm{~d}, J_{C F}=25.6 \mathrm{~Hz}\right), 125.2,129.3,129.9,130.2,132.1,132.3$, $139.7,140.6,149.4,156.2,157.6\left(\mathrm{~d}, J_{C F}=243.5 \mathrm{~Hz}\right), 166.0,170.6,178.4,191.8 ;{ }^{19} \mathrm{~F} \mathrm{NMR}$ $\left(\mathrm{CDCl}_{3}, 376 \mathrm{MHz}\right) \delta$ : -120.19; HRMS (ESI-TOF) m/z: Calcd. for $\mathrm{C}_{39} \mathrm{H}_{36} \mathrm{BrFN}_{2} \mathrm{NaO}_{9}[\mathrm{M}+\mathrm{Na}]^{+}$: 797.1480; Found: 797.1481.

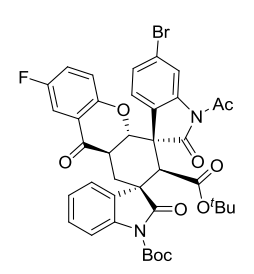

3v: White solid, m.p. $130.3-132.2{ }^{\circ} \mathrm{C} ; 67.3 \mathrm{mg}$, yield $87 \%$; >99\% ee, $>20: 1 \mathrm{dr},[\alpha]_{\mathrm{D}}{ }^{20}=+138.0(\mathrm{c}$ 0.25, $\mathrm{CH}_{2} \mathrm{Cl}_{2}$ ); The ee was determined by HPLC analysis using a Chiralpak IC column $(98 / 2$ hexane $/ i-\mathrm{PrOH}$; flow rate: $1.0 \mathrm{~mL} / \mathrm{min} ; \lambda=254 \mathrm{~nm} ; \tau_{\text {major }}=13.96 \mathrm{~min} ; \tau_{\text {minor }}=27.88 \mathrm{~min}$ ); ${ }^{1} \mathrm{H}$ NMR $\left(\mathrm{CDCl}_{3}, 400 \mathrm{MHz}\right) \delta$ : 0.71 (s, 9H), $1.66(\mathrm{~s}, 9 \mathrm{H}), 2.45-2.54(\mathrm{~m}, 1 \mathrm{H}), 2.62-2.68(\mathrm{~m}, 1 \mathrm{H}), 2.73$ (s, 3H), 3.83-3.91 (m, 1H), $4.01(\mathrm{~s}, 1 \mathrm{H}), 5.83(\mathrm{~d}, J=14.0 \mathrm{~Hz}, 1 \mathrm{H}), 6.69-6.72(\mathrm{~m}, 1 \mathrm{H}), 7.08-7.13$ $(\mathrm{m}, 1 \mathrm{H}), 7.17-7.21(\mathrm{~m}, 1 \mathrm{H}), 7.28-7.37(\mathrm{~m}, 3 \mathrm{H}), 7.44-7.47(\mathrm{~m}, 1 \mathrm{H}), 7.74-7.78(\mathrm{~m}, 2 \mathrm{H}), 8.50(\mathrm{~d}, J=$ $1.6 \mathrm{~Hz}, 1 \mathrm{H}) ;{ }^{13} \mathrm{C} \mathrm{NMR}\left(\mathrm{CDCl}_{3}, 100 \mathrm{MHz}\right) \delta: 26.8,26.9,28.2,38.8,47.6,53.9,56.7,81.0,83.0$, $84.9,112.3\left(\mathrm{~d}, J_{C F}=23.5 \mathrm{~Hz}\right), 112.4,115.2,119.1,120.9\left(\mathrm{~d}, J_{C F}=7.1 \mathrm{~Hz}\right), 121.6,123.2,123.7(\mathrm{~d}$, $\left.J_{C F}=25.1 \mathrm{~Hz}\right), 125.1,126.9,128.2,128.7,129.1,132.5,139.4,142.4,149.0,156.2,157.6\left(\mathrm{~d}, J_{C F}\right.$ $=242.0 \mathrm{~Hz}), 166.0,170.6,178.9,191.8 ;{ }^{19} \mathrm{~F} \mathrm{NMR}\left(\mathrm{CDCl}_{3}, 376 \mathrm{MHz}\right) \delta:-120.17$; HRMS (ESI-TOF) m/z: Calcd. for $\mathrm{C}_{39} \mathrm{H}_{36} \mathrm{BrFN}_{2} \mathrm{NaO}_{9}[\mathrm{M}+\mathrm{Na}]^{+}$: 797.1480; Found: 797.1484.

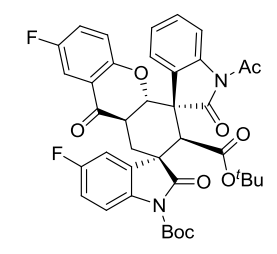

3w: White solid, m.p. $134.2-135.8{ }^{\circ} \mathrm{C} ; 57.8 \mathrm{mg}$, yield $81 \%$; $94 \%$ ee, $>20: 1 \mathrm{dr},[\alpha]_{\mathrm{D}}{ }^{20}=+130.6(c$ 
0.33, $\mathrm{CH}_{2} \mathrm{Cl}_{2}$ ); The ee was determined by HPLC analysis using a Chiralpak IA column (98/2 hexane $/ i$-PrOH; flow rate: $\left.1.0 \mathrm{~mL} / \mathrm{min} ; \lambda=254 \mathrm{~nm} ; \tau_{\text {major }}=24.33 \mathrm{~min} ; \tau_{\text {minor }}=15.57 \mathrm{~min}\right) ;{ }^{1} \mathrm{H}$ NMR $\left(\mathrm{CDCl}_{3}, 400 \mathrm{MHz}\right) \delta: 0.71(\mathrm{~s}, 9 \mathrm{H}), 1.68(\mathrm{~s}, 9 \mathrm{H}), 2.43-2.50(\mathrm{~m}, 1 \mathrm{H}), 2.67-2.73(\mathrm{~m}, 1 \mathrm{H}), 2.76$ (s, 3H), 3.87-3.95 (m, 1H), 3.99 (s, 1H), $5.87(\mathrm{~d}, J=14.4 \mathrm{~Hz}, 1 \mathrm{H}), 6.67-6.71(\mathrm{~m}, 1 \mathrm{H}), 6.99-7.04$ (m, 1H), 7.07-7.12 (m, 2H), 7.20-7.24 (m, 1H), 7.35-7.39 (m, 1H), 7.46-7.49 (m, 1H), 7.79-7.82 (m, 1H), 7.83-7.85 (m, 1H), $8.29(\mathrm{~d}, J=8.4 \mathrm{~Hz}, 1 \mathrm{H}) ;{ }^{13} \mathrm{C} \mathrm{NMR}\left(\mathrm{CDCl}_{3}, 100 \mathrm{MHz}\right) \delta: 26.7,28.2$, 31.6, 38.7, 47.8, 53.8, 56.7, 81.0, 82.8, 84.8, $109.2\left(\mathrm{~d}, J_{C F}=25.1 \mathrm{~Hz}\right), 112.2\left(\mathrm{~d}, J_{C F}=24.7 \mathrm{~Hz}\right)$, $115.4\left(\mathrm{~d}, J_{C F}=23.8 \mathrm{~Hz}\right), 115.8,116.7\left(\mathrm{~d}, J_{C F}=7.1 \mathrm{~Hz}\right), 119.6,120.9\left(\mathrm{~d}, J_{C F}=6.9 \mathrm{~Hz}\right), 123.5(\mathrm{~d}$, $\left.J_{C F}=24.4 \mathrm{~Hz}\right), 125.7,126.7,127.8,129.4,134.5\left(\mathrm{~d}, J_{C F}=7.5 \mathrm{~Hz}\right), 135.4,141.5,148.9,156.3$, 158.7, $160.3\left(\mathrm{~d}, J_{C F}=244.6 \mathrm{~Hz}\right), 165.9,170.6,178.3,179.3,191.7 ;{ }^{19} \mathrm{~F} \mathrm{NMR}\left(\mathrm{CDCl}_{3}, 376 \mathrm{MHz}\right)$ $\delta$ : -116.60, -120.37; HRMS (ESI-TOF) m/z: Calcd. for $\mathrm{C}_{39} \mathrm{H}_{36} \mathrm{~F}_{2} \mathrm{~N}_{2} \mathrm{NaO}_{9}[\mathrm{M}+\mathrm{Na}]^{+}$: 737.2281; Found: 737.2277 .

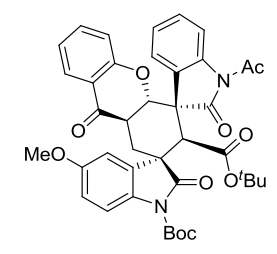

3x: White solid, m.p. $134.6-136.3{ }^{\circ} \mathrm{C} ; 46.0 \mathrm{mg}$, yield $65 \%$; $94 \%$ ee, $>20: 1 \mathrm{dr},[\alpha]_{\mathrm{D}}{ }^{20}=+95.7(c$ $0.30, \mathrm{CH}_{2} \mathrm{Cl}_{2}$ ); The ee was determined by HPLC analysis using a Chiralpak IA column $(95 / 5$ hexane $/ \mathrm{i}-\mathrm{PrOH}$; flow rate: $1.0 \mathrm{~mL} / \mathrm{min} ; \lambda=254 \mathrm{~nm} ; \tau_{\text {major }}=30.66 \mathrm{~min} ; \tau_{\text {minor }}=16.44 \mathrm{~min}$ ); ${ }^{1} \mathrm{H}$ NMR $\left(\mathrm{CDCl}_{3}, 400 \mathrm{MHz}\right) \delta$ : $0.71(\mathrm{~s}, 9 \mathrm{H}), 1.67(\mathrm{~s}, 9 \mathrm{H}), 2.45-2.51(\mathrm{~m}, 1 \mathrm{H}), 2.68-2.74(\mathrm{~m}, 1 \mathrm{H}), 2.76$ (s, 3H), $3.82(\mathrm{~s}, 3 \mathrm{H}), 3.87-3.96(\mathrm{~m}, 1 \mathrm{H}), 4.03(\mathrm{~s}, 1 \mathrm{H}), 5.91$ (d, $J=14.0 \mathrm{~Hz}, 1 \mathrm{H}), 6.69$ (d, $J=8.4$ $\mathrm{Hz}, 1 \mathrm{H}), 6.82-6.85(\mathrm{~m}, 1 \mathrm{H}), 6.88(\mathrm{~s}, 1 \mathrm{H}), 6.97-7.00(\mathrm{~m}, 1 \mathrm{H})$, 7.20-7.23 (m, 1H), 7.34-7.39 (m, 2H), $7.71(\mathrm{~d}, J=8.8 \mathrm{~Hz}, 1 \mathrm{H}), 7.81-7.84(\mathrm{~m}, 1 \mathrm{H}), 7.88-7.90(\mathrm{~m}, 1 \mathrm{H}), 8.29(\mathrm{~d}, J=8.0 \mathrm{~Hz}, 1 \mathrm{H}) ;{ }^{13} \mathrm{C} \mathrm{NMR}$ $\left(\mathrm{CDCl}_{3}, 100 \mathrm{MHz}\right) \delta: 26.8,28.2,31.9,38.9,47.9,54.1,56.0,56.7,80.9,82.6,84.4,108.0,113.6$, 115.7, 116.1, 117.9, 120.4, 122.0, 125.6, 126.8, 127.2, 128.0, 129.2, 132.7, 134.1, 136.0, 141.5, 149.1, 157.4, 160.1, 166.2, 170.7, 178.7, 179.5, 192.6; HRMS (ESI-TOF) m/z: Calcd. for $\mathrm{C}_{40} \mathrm{H}_{40} \mathrm{~N}_{2} \mathrm{NaO}_{10}[\mathrm{M}+\mathrm{Na}]^{+}:$731.2575; Found: 731.2579. 


\section{Gram scale synthesis of the product $3 d$}

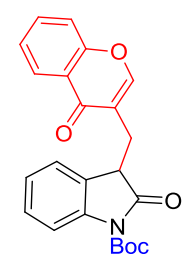

$1(2.0 \mathrm{mmol})$

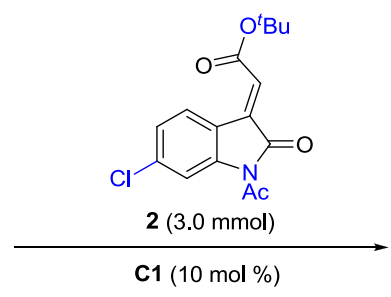

$\mathrm{Et}_{2} \mathrm{O}, 5 \AA \mathrm{MS}, \mathrm{rt}, 4 \mathrm{~d}$

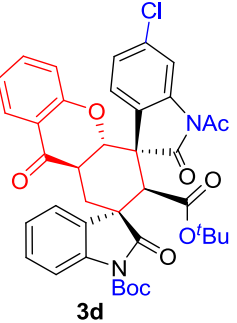

3d

In a sealed tube equipped with a magnetic stirring bar, to the mixture of oxindole-chromone synthon $1(2.0 \mathrm{mmol}), 5 \AA$ MS (600 mg) and quinine-derived thiourea catalyst $\mathbf{C 1}(10 \mathrm{~mol} \%)$ in $35 \mathrm{~mL}$ of freshly distilled $\mathrm{Et}_{2} \mathrm{O}$ was added methyleneindolinone $2(3.0 \mathrm{mmol})$. The reaction mixture was stirred at $\mathrm{rt}$ for $4 \mathrm{~d}$ and was directly loaded onto a silica gel and purified by flash chromatography to give the desired product 3d, using hexane/EtOAc $(10 / 1, \mathrm{v} / \mathrm{v})$ as the eluent $(1.13 \mathrm{~g}, 80 \%, 98 \%$ ee, $>20: 1 \mathrm{dr})$. 

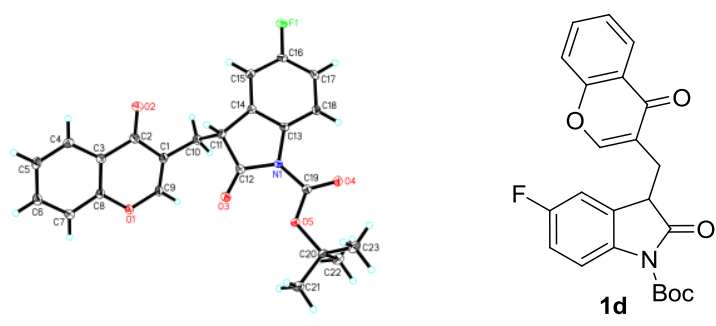

Table S2 Crystal data and structure refinement for 1d

Identification code

Empirical formula

Formula weight

Temperature/K

Crystal system

Space group

$\mathrm{a} / \AA ̊ \mathrm{~A}, \mathrm{~b} / \mathrm{A}, \mathrm{c} / \AA ̊ \AA$

$\alpha /^{\circ}, \beta /^{\circ}, \gamma /^{\circ}$

Volume $/ \AA^{3}$

$\mathrm{Z}$

$\rho_{\text {calc }} \mathrm{g} / \mathrm{cm}^{3}$

$\mu / \mathrm{mm}^{-1}$

$\mathrm{F}(000)$

Crystal size $/ \mathrm{mm}^{3}$

Radiation

$2 \Theta$ range for data collection $/{ }^{\circ}$

Index ranges

Reflections collected

Independent reflections

Data/restraints/parameters

Goodness-of-fit on $\mathrm{F}^{2}$

Final $R$ indexes $[I>=2 \sigma(I)]$

Final $\mathrm{R}$ indexes [all data]

Largest diff. peak/hole / e $\AA^{-3}$ 1d

$\mathrm{C}_{23} \mathrm{H}_{20} \mathrm{FNO}_{5}$

409.40

100.00(10)

monoclinic

$\mathrm{P} 2{ }_{1} / \mathrm{n}$

13.439(3), 10.5456(17), 15.128(3)

90, 114.48(3), 90.

1951.3(8)

4

1.394

0.105

856.0

$0.13 \times 0.12 \times 0.11$

$\operatorname{MoK} \alpha(\lambda=0.71073)$

4.866 to 50

$-15 \leq \mathrm{h} \leq 12,-12 \leq \mathrm{k} \leq 11,-15 \leq 1 \leq 17$

8801

$3371\left[R_{\text {int }}=0.0380, R_{\text {sigma }}=0.0486\right]$

$3371 / 0 / 274$

1.053

$\mathrm{R}_{1}=0.0447, \mathrm{wR}_{2}=0.0989$

$\mathrm{R}_{1}=0.0561, \mathrm{wR}_{2}=0.1069$

$0.30 /-0.22$

\section{Crystal structure determination of 1d}

Crystal Data for $\mathrm{C}_{23} \mathrm{H}_{20} \mathrm{FNO}_{5}(M=409.40 \mathrm{~g} / \mathrm{mol}$ ): monoclinic, space group $\mathrm{P} 21 / \mathrm{n}$ (no. 14), $a=$ 13.439(3) $\mathrm{A}, b=10.5456(17) \AA, c=15.128(3) \AA, \beta=114.48(3)^{\circ}, V=1951.3(8) \AA^{3}, Z=4, T=$ $100.00(10) \mathrm{K}, \mu(\mathrm{MoK} \alpha)=0.105 \mathrm{~mm}^{-1}$, Dcalc $=1.394 \mathrm{~g} / \mathrm{cm}^{3}, 8801$ reflections measured $\left(4.866^{\circ} \leq\right.$ $\left.2 \Theta \leq 50^{\circ}\right), 3371$ unique $\left(R_{\text {int }}=0.0380, R_{\text {sigma }}=0.0486\right)$ which were used in all calculations. The final $R_{1}$ was $0.0447(\mathrm{I}>2 \sigma(\mathrm{I}))$ and $w R_{2}$ was 0.1069 (all data). 

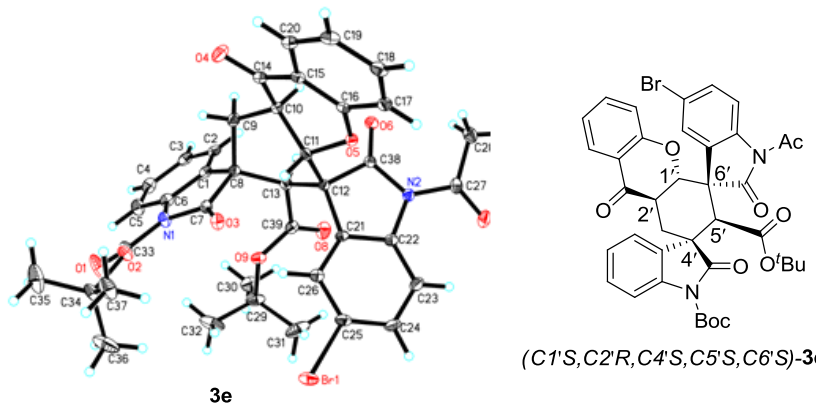

(C1'S, C2'R, C4'S, C5'S, C6'S)-3e

Table S3 Crystal data and structure refinement for $3 e$

Identification code

Empirical formula

Formula weight

Temperature/K

Crystal system

Space group

$\mathrm{a} / \AA ̊ \mathrm{~A}, \mathrm{~b} / \AA, \mathrm{c} / \AA$

$\alpha /^{\circ}, \beta /{ }^{\circ}, \gamma /{ }^{\circ}$,

Volume $/ \AA^{3}$

Z

$\rho_{\text {calc }} \mathrm{g} / \mathrm{cm}^{3}$

$\mu / \mathrm{mm}^{-1}$

$\mathrm{F}(000)$

Crystal size $/ \mathrm{mm}^{3}$

Radiation

$2 \Theta$ range for data collection ${ }^{\circ}$

Index ranges

Reflections collected

Independent reflections

Data/restraints/parameters

Goodness-of-fit on $\mathrm{F}^{2}$

Final $\mathrm{R}$ indexes $[\mathrm{I}>=2 \sigma(\mathrm{I})]$

Final $\mathrm{R}$ indexes [all data]

Largest diff. peak/hole / e $\AA^{-3}$

Flack parameter
$3 \mathbf{e}$

$\mathrm{C}_{39} \mathrm{H}_{37} \mathrm{BrN}_{2} \mathrm{O}_{9}$

757.61

100.00(10)

orthorhombic

$\mathrm{C} 222_{1}$

11.75523(14), 21.1760(3), 29.6689(3)

90, 90, 90 .

7385.44(15)

8

1.363

1.994

3136.0

$0.13 \times 0.12 \times 0.11$

$\mathrm{CuK} \alpha(\lambda=1.54184)$

5.958 to 147.258

$-9 \leq \mathrm{h} \leq 14,-25 \leq \mathrm{k} \leq 22,-25 \leq 1 \leq 36$

20421

$7286\left[\mathrm{R}_{\text {int }}=0.0239, \mathrm{R}_{\text {sigma }}=0.0221\right]$

$7286 / 0 / 467$

1.040

$\mathrm{R}_{1}=0.0319, \mathrm{wR}_{2}=0.0842$

$\mathrm{R}_{1}=0.0322, \mathrm{wR}_{2}=0.0845$

$0.73 /-0.55$

$-0.018(5)$

\section{Crystal structure determination of $3 \mathrm{e}$}

Crystal Data for $\mathrm{C}_{39} \mathrm{H}_{37} \mathrm{BrN}_{2} \mathrm{O}_{9}(M=757.61 \mathrm{~g} / \mathrm{mol}$ ): orthorhombic, space group C222 1 (no. 20), $a=11.75523(14) \AA, b=21.1760(3) \AA, c=29.6689(3) \AA, V=7385.44(15) \AA^{3}, Z=8, T=$ $100.00(10) \mathrm{K}, \mu(\mathrm{CuK \alpha})=1.994 \mathrm{~mm}^{-1}$, Dcalc $=1.363 \mathrm{~g} / \mathrm{cm}^{3}, 20421$ reflections measured $\left(5.958^{\circ} \leq\right.$ $\left.2 \Theta \leq 147.258^{\circ}\right), 7286$ unique $\left(R_{\text {int }}=0.0239, R_{\text {sigma }}=0.0221\right)$ which were used in all calculations. The final $R_{1}$ was $0.0319(\mathrm{I}>2 \sigma(\mathrm{I}))$ and $w R_{2}$ was 0.0845 (all data). 

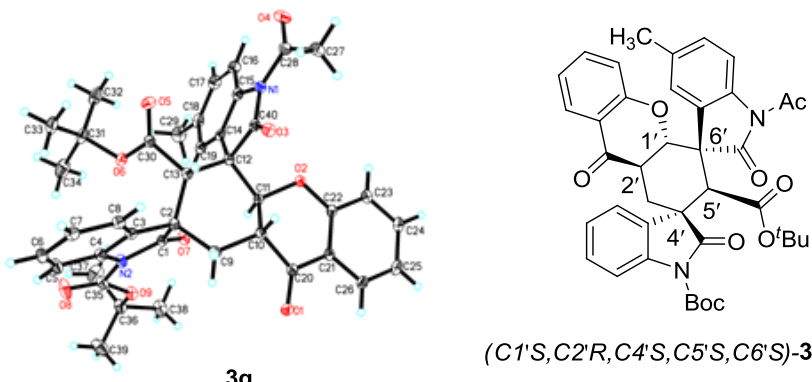

(C1'S, C2'R, C4'S, C5'S, C6'S)-3g

$3 g$

Table S4 Crystal data and structure refinement for $\mathbf{3 g}$

Identification code

$3 g$

Empirical formula

$\mathrm{C}_{40} \mathrm{H}_{40} \mathrm{~N}_{2} \mathrm{O}_{9}$

Formula weight

692.74

Temperature/K

$100.00(10)$

Crystal system

orthorhombic

Space group

$\mathrm{C} 222_{1}$

$\mathrm{a} / \AA ̊ \mathrm{,} \mathrm{b} / \AA ̊ \cap, c / \AA$

11.65556(13), 21.16199(18), 29.9310(2)

$\alpha /^{\circ}, \beta /{ }^{\circ}, \gamma /{ }^{\circ}$

90, 90, 90 .

Volume $/ \AA^{3}$

7382.62(12)

$\mathrm{Z}$

8

$\rho_{\text {calc } g} / \mathrm{cm}^{3}$

1.247

$\mu / \mathrm{mm}^{-1}$

0.726

$\mathrm{F}(000)$

2928.0

Crystal size $/ \mathrm{mm}^{3}$

$0.16 \times 0.13 \times 0.12$

Radiation

$\mathrm{CuK} \alpha(\lambda=1.54184)$

$2 \Theta$ range for data collection ${ }^{\circ}$

5.906 to 147.878

Index ranges

$-11 \leq \mathrm{h} \leq 14,-25 \leq \mathrm{k} \leq 26,-37 \leq 1 \leq 33$

Reflections collected

22378

Independent reflections

$7329\left[\mathrm{R}_{\mathrm{int}}=0.0316, \mathrm{R}_{\text {sigma }}=0.0252\right]$

Data/restraints/parameters

$7329 / 0 / 468$

Goodness-of-fit on $\mathrm{F}^{2}$

1.068

Final $\mathrm{R}$ indexes $[\mathrm{I}>=2 \sigma(\mathrm{I})]$

$\mathrm{R}_{1}=0.0350, \mathrm{wR}_{2}=0.0906$

Final $\mathrm{R}$ indexes [all data]

$\mathrm{R}_{1}=0.0360, \mathrm{wR}_{2}=0.0911$

Largest diff. peak/hole / e $\AA^{-3}$

$0.19 /-0.23$

Flack/Hooft parameter

$0.03(6) / 0.05(5)$

Crystal structure determination of $3 \mathrm{~g}$

Crystal Data for $\mathrm{C}_{40} \mathrm{H}_{40} \mathrm{~N}_{2} \mathrm{O}_{9}(M=692.74 \mathrm{~g} / \mathrm{mol}$ ): orthorhombic, space group C222 1 (no. 20), $a=$ 11.65556(13) $\AA, b=\quad 21.16199(18) \AA, c=\quad 29.9310(2) \AA, V=\quad 7382.62(12) \AA^{3}, Z=\quad 8, T=$ $100.00(10) \mathrm{K}, \mu(\mathrm{CuK \alpha})=0.726 \mathrm{~mm}^{-1}$, Dcalc $=1.247 \mathrm{~g} / \mathrm{cm}^{3}, 22378$ reflections measured $\left(5.906^{\circ} \leq\right.$ $\left.2 \Theta \leq 147.878^{\circ}\right), 7329$ unique $\left(R_{\text {int }}=0.0316, R_{\text {sigma }}=0.0252\right)$ which were used in all calculations. The final $R_{1}$ was $0.0350(\mathrm{I}>2 \sigma(\mathrm{I}))$ and $w R_{2}$ was 0.0911 (all data). 


\section{A Plausible Reaction Mechanism}

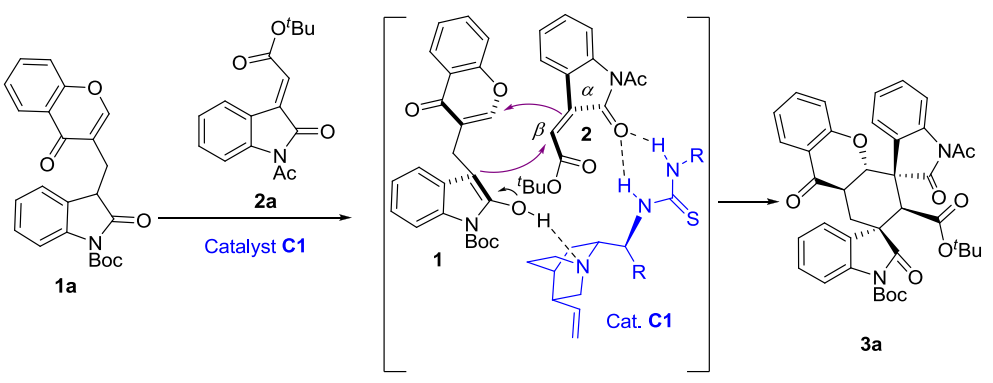

On the basis of the absolute configuration of chiral $\mathbf{3 e}$ and $\mathbf{3 g}$, and previous related literature, ${ }^{4,5}$ we have proposed a plausible reaction mechanism to account for the observed stereochemistry. In this model, oxindole 1a is deprotonated by the basic nitrogen atom of the tertiary amine in catalyst C1. Meanwhile, methyleneindolinone $\mathbf{2 a}$ is oriented and activated by the thiourea moiety through double hydrogen bonding interactions. Subsequently, the $\beta$-position of the electrophile substrate 2a undergoes nucleophilic attack at the $\mathrm{Si}$-face by the activated enolated substrate 1a from the Si-face under the catalyst $\mathbf{C 1}$ control by efficient shielding effect. Finally, intramolecular annulation process through the carbanion of $\alpha$-position of substrate $\mathbf{2 a}$ attack to the $\mathrm{Si}$-face of the electron-defficient chromone moiety leads to the formation of $\left(\mathrm{C} 1 ' S, \mathrm{C} 2{ }^{\prime} R, \mathrm{C} 4{ }^{\prime} S, \mathrm{C} 5^{\prime} S\right.$, C6'S)-configured 3a.

\section{General Experimental Procedures for In Vitro Cytotoxicity Assay}

Three human cancer cell lines, human leukemia cells K562, human lung cancer cells A549 and human prostate cancer cells PC-3 were purchased from Chinese Academy of Sciences, Kunming Cell Bank and Chinese Academy of Sciences, Shanghai Cell Bank respectively. All the cells were cultured in RPMI-1640 medium (GIBICO, USA), supplemented with $10 \%$ fetal bovine serum (Hyclone, USA) and Penicillin-Streptomycin (respectively $100 \mathrm{U} / \mathrm{mL}$ ) in $5 \% \mathrm{CO}_{2}$ at $37^{\circ} \mathrm{C}$. The cytotoxicity assay was performed according to the MTT (3-(4,5-dimethylthiazol-2-yl)-2, 5-diphenyl tetrazolium bromide) method in 96-well microplates. Briefly, 5000 cells were seeded into each well of 96-well cell culture plates and allowed to grow for $24 \mathrm{~h}$ before drug addition. Unless K562 tumor cell line was exposed to compounds (3a, 3c, 3e, 3j, 3o, 3q, 3r, 3w and $\mathbf{3 x})$ at the concentrations of $1,2,4,8$ and $20 \mu \mathrm{mol} \cdot \mathrm{L}^{-1}$, each A549 or PC-3 tumor cell line was exposed to the test compounds (3a, 3c, 3e, 3j, 3o, 3q, 3r, 3w and $\mathbf{3 x}$ ) at the concentrations of 5, 10, 20, 40 and $80 \mu \mathrm{mol} \cdot \mathrm{L}^{-1}$ in triplicates for $48 \mathrm{~h}$, comparable to cisplatin (Aladdin, China). Then the MTT 
reagent was added to reaction with the cancer cells for 4 hours. At least, measure the OD value at 490 wavelengths. The average $50 \%$ inhibitory concentration $\left(\mathrm{IC}_{50}\right)$ of all the compounds were calculated by IBM SPSS Statistics (version 19). Each concentration was analyzed in triplicate at least, and the whole experiment was repeated three times.

\section{General Experimental Procedures for Flow Cytometry Analysis of Apoptotic Analysis}

$4 \times 10^{5}$ cells were seeded per well into a 6-wells plate (Nest Biotechnology, China). After $12 \mathrm{~h}$ incubation, replaced with fresh medium containing the compound 3a at different concentrations for another $36 \mathrm{~h}$. Then, the cells were harvested and the Annexin-VFITC/PI apoptosis detection kit (CoWin Biosciences, China) was used according to the instructions to detect the apoptosis cells. Ten thousand events were collected for each sample and analyzed by flow cytometry (BD Bioscience, USA).

\section{General Experimental Procedures for AO-EB Double Staining}

$4 \times 10^{5}$ cells were seeded per well into a 6-wells plate (Nest Biotechnology, China). After $12 \mathrm{~h}$ incubation, replaced with fresh medium containing the compound 3a at different concentrations for another $36 \mathrm{~h}$. Then, removed the medium, The cells were washed twice with ice-cold PBS, and then stained with $1 \mathrm{~mL}$ of dye mixture containing $100 \mu \mathrm{g} / \mathrm{mL} \mathbf{A O}$ and $1 \mathrm{mg} / \mathrm{mL} \mathbf{E B}$ in PBS and incubated at room temperature for $5 \mathrm{~min}$ in the dark. The stained cells were observed under fluorescence microscope (Leica Inc., Germany) at $10 \times$ magnification.

\section{REFERENCES}

(1) Huang, A.; Kodanko, J. J.; Overman, L. E. J. Am. Chem. Soc. 2004, 126, 14043-14053.

(2) Hamashima, Y.; Suzuki, T.; Takano, H.; Shimura, Y.; Sedeoka, M. J. Am. Chem. Soc. 2005, 127, 10164-10165.

(3) Haas, G.; Stanton, J. L.; Sprecher, A.; Wenk, P. Journal of Heterocyclic Chemistry 1981, 18, 607-612.

(4) Taylor, M. S.; Jacobsen, E. N. Angew. Chem. Int. Ed. 2006, 45, 1520-1543.

(5) Schreiner, P. R. Chem. Soc. Rev. 2003, 32, 289-296. 
13. Figure S1: Compound 3a Induced Apoptosis of K562 Cells.

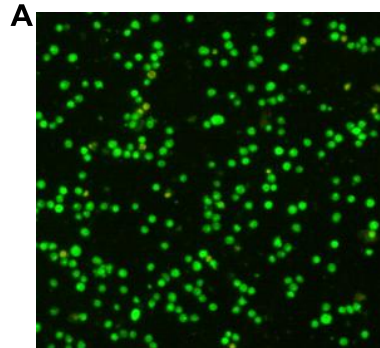

Control

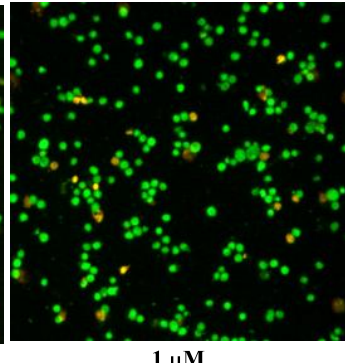

$1 \mu \mathbf{M}$

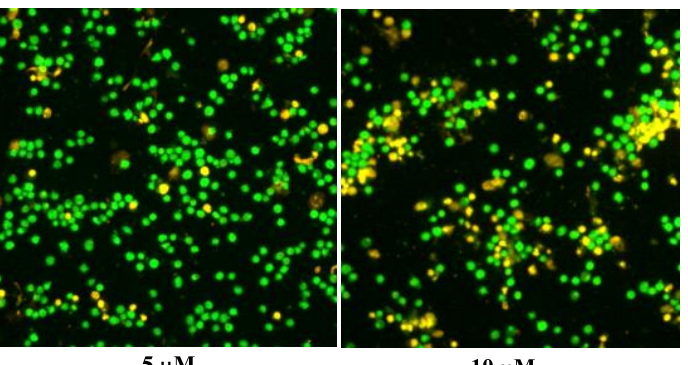

$10 \mu \mathbf{M}$

B
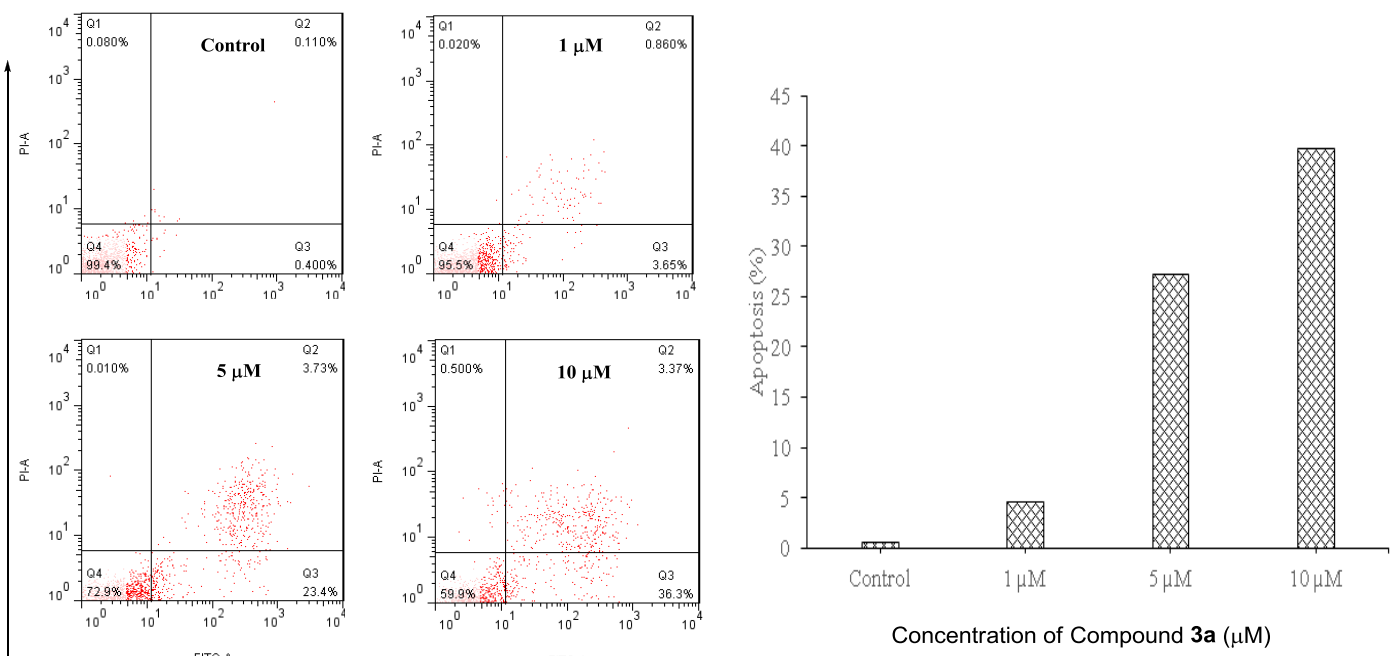

(A) Apoptosis analysis with AO/EB staining; (B) Quantitative analysis of apoptotic cells using Annexin V-FITC/PI double staining and flow cytometry calculation. The lower left quadrant represents live cells, the lower right is for early/primary apoptotic cells, upper right is for late/secondary apoptotic cells, and the upper left represents cells damaged during the procedure. 
14. The Copies of ${ }^{1} \mathrm{H}$ NMR, ${ }^{13} \mathrm{C}$ NMR and HPLC Spectra for Compounds 1 and 3 ${ }^{1} \mathrm{H}$ and ${ }^{13} \mathrm{C}$ NMR of $1 \mathrm{a}$
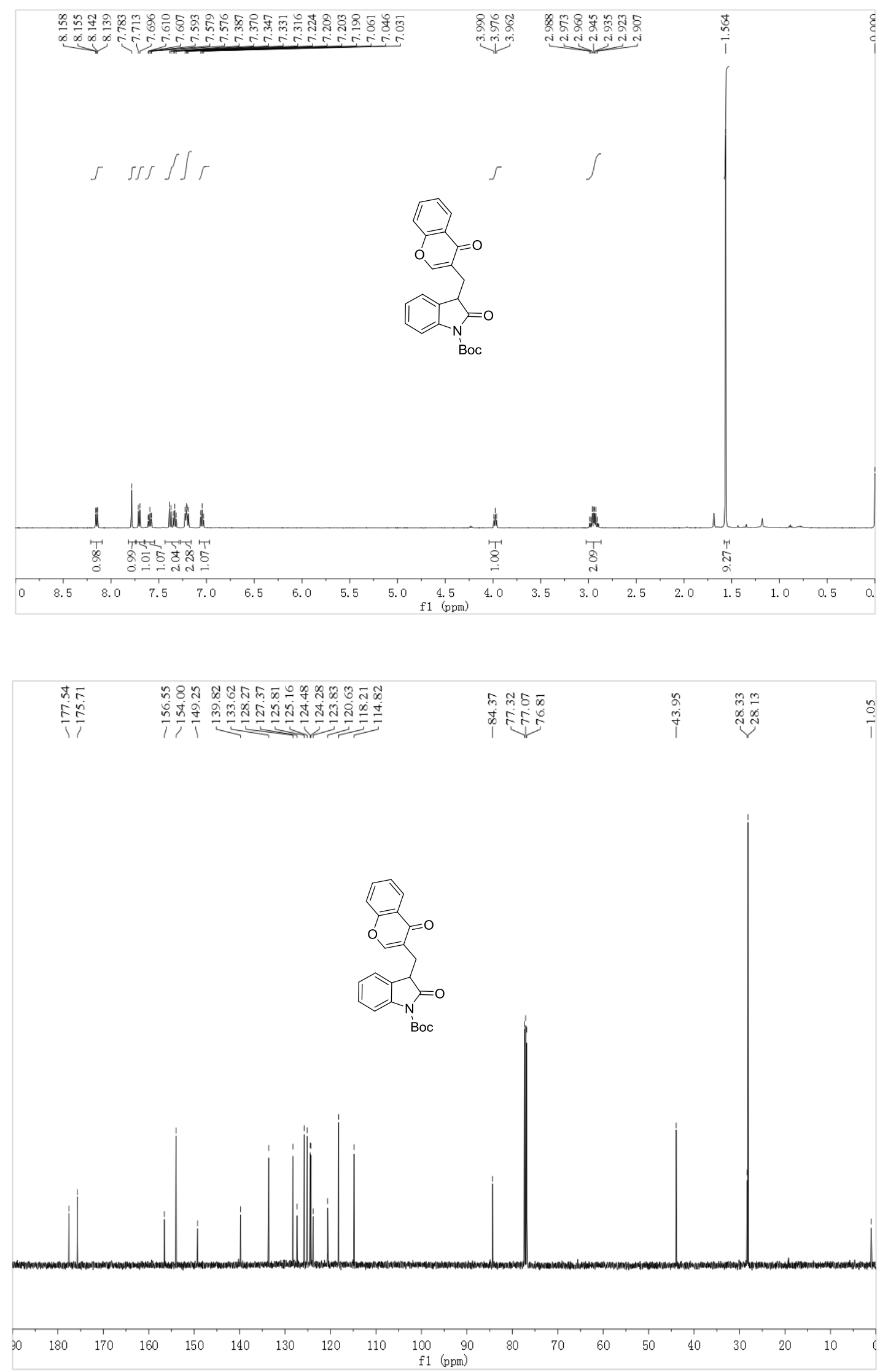
${ }^{1} \mathrm{H}$ and ${ }^{13} \mathrm{C}$ NMR of $1 \mathrm{~b}$
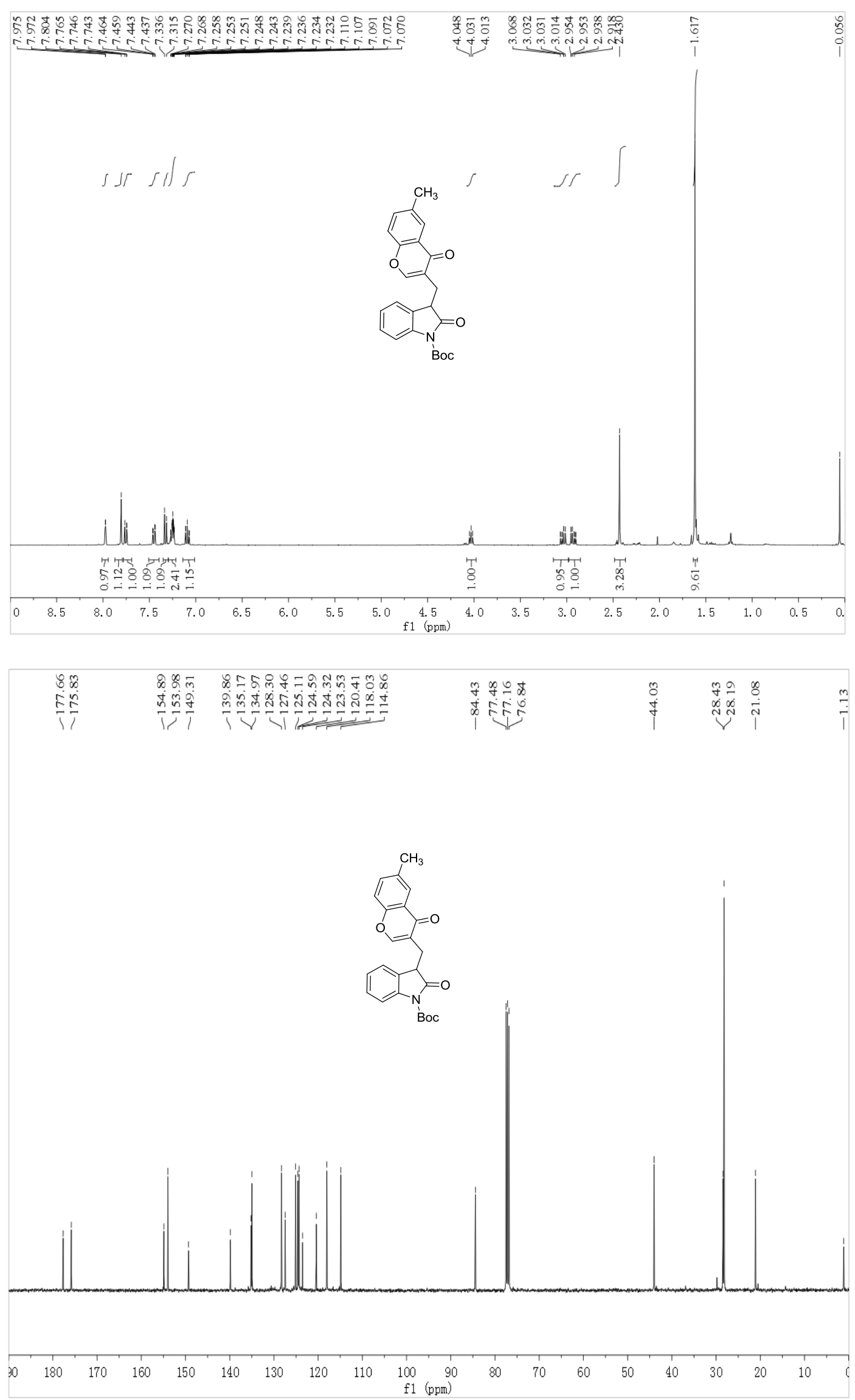
${ }^{1} \mathrm{H}$ and ${ }^{13} \mathrm{C}$ NMR of $1 \mathrm{c}$
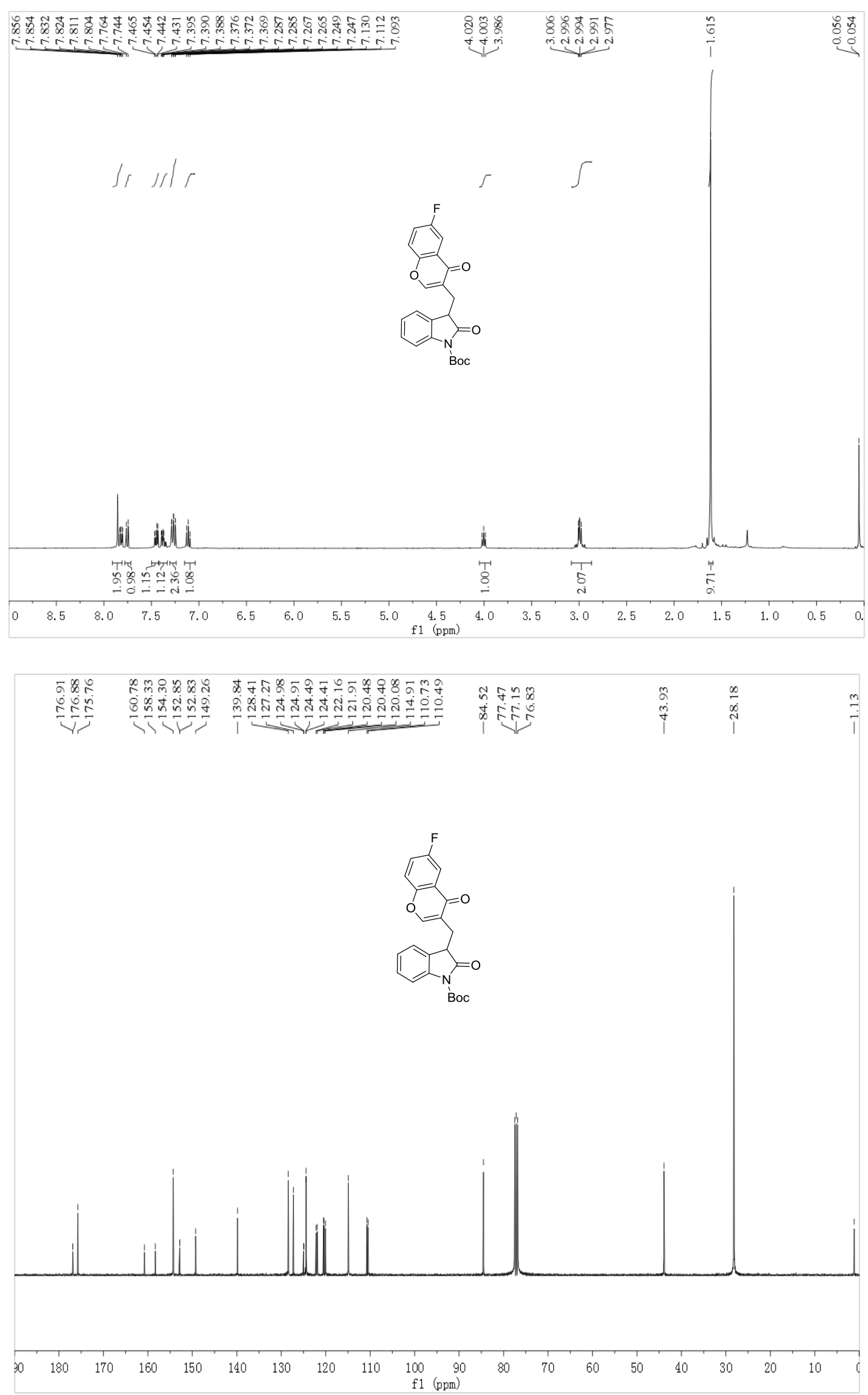
${ }^{19}$ F NMR of 1c

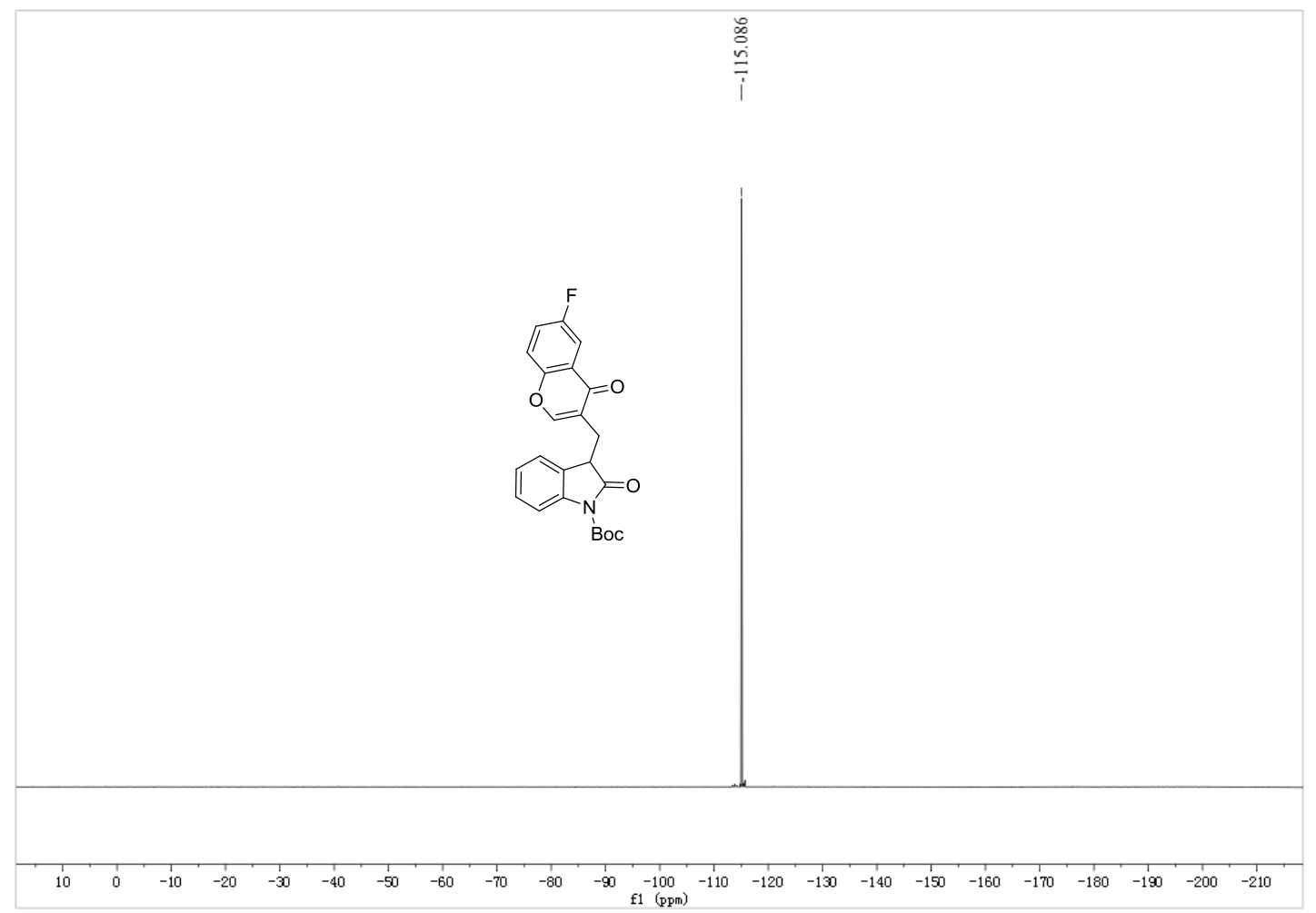


${ }^{1} \mathrm{H}$ and ${ }^{13} \mathrm{C}$ NMR of $1 \mathrm{~d}$
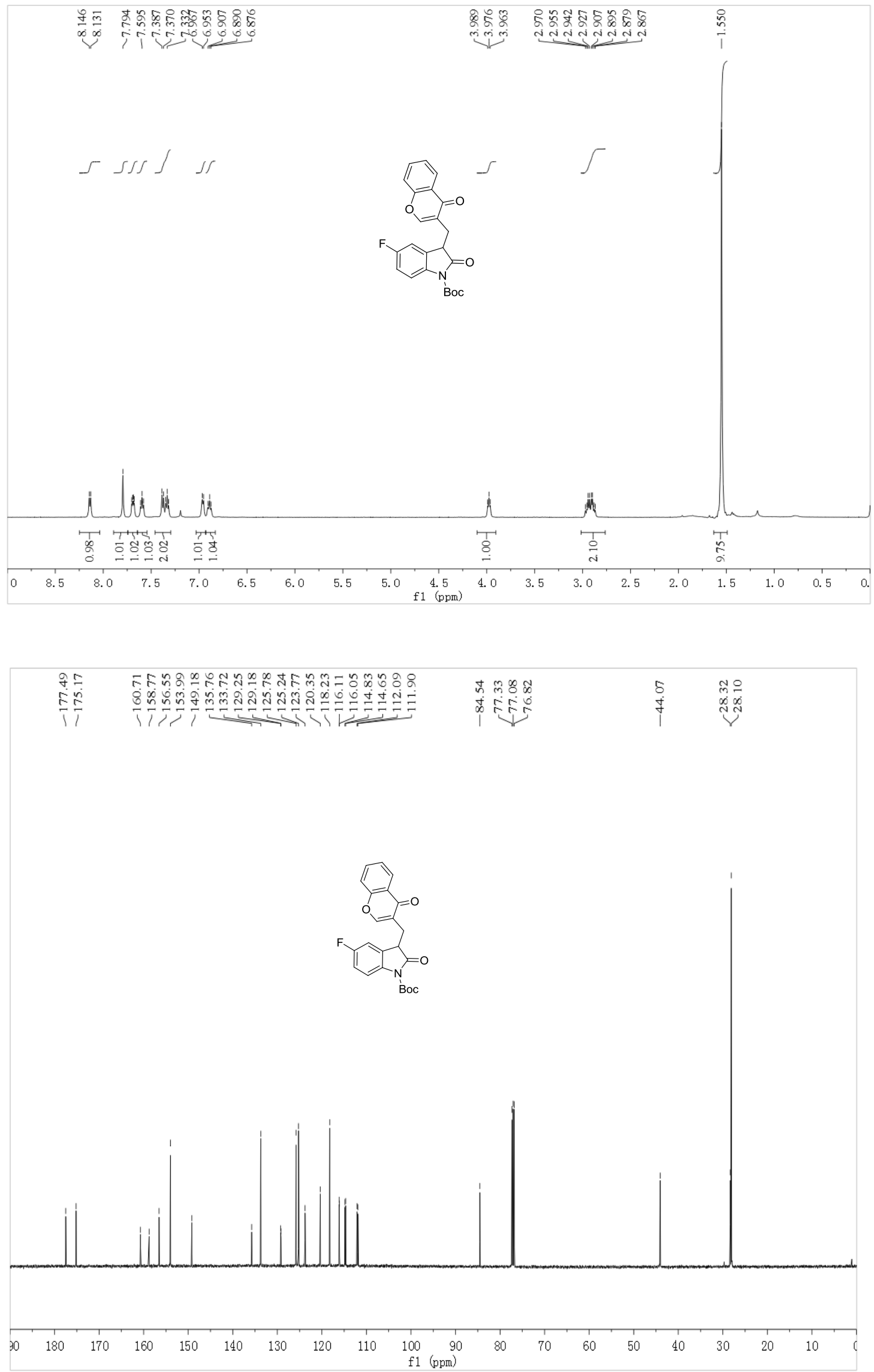
${ }^{19}$ F NMR of 1d

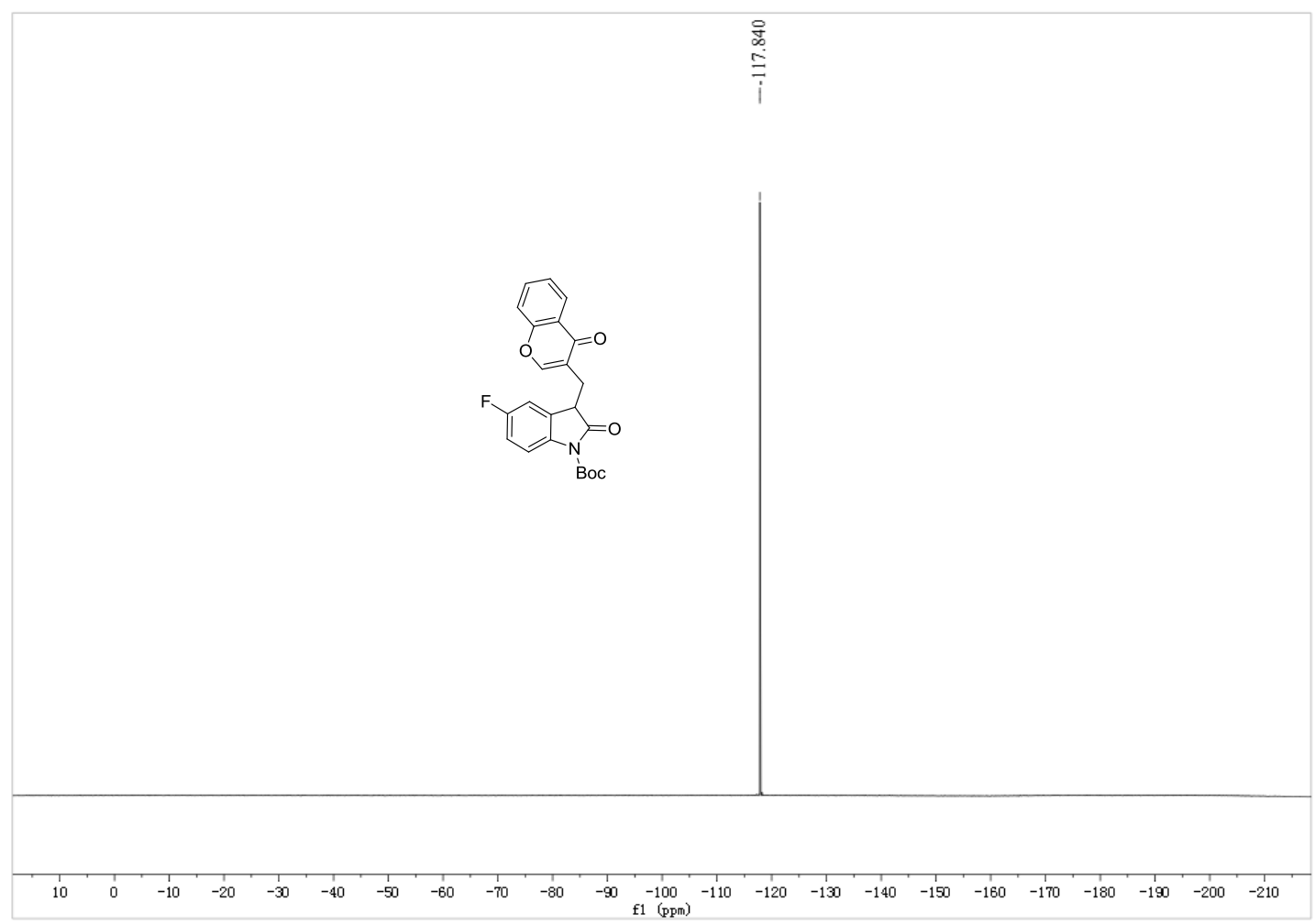


${ }^{1} \mathrm{H}$ and ${ }^{13} \mathrm{C}$ NMR of $1 \mathrm{e}$
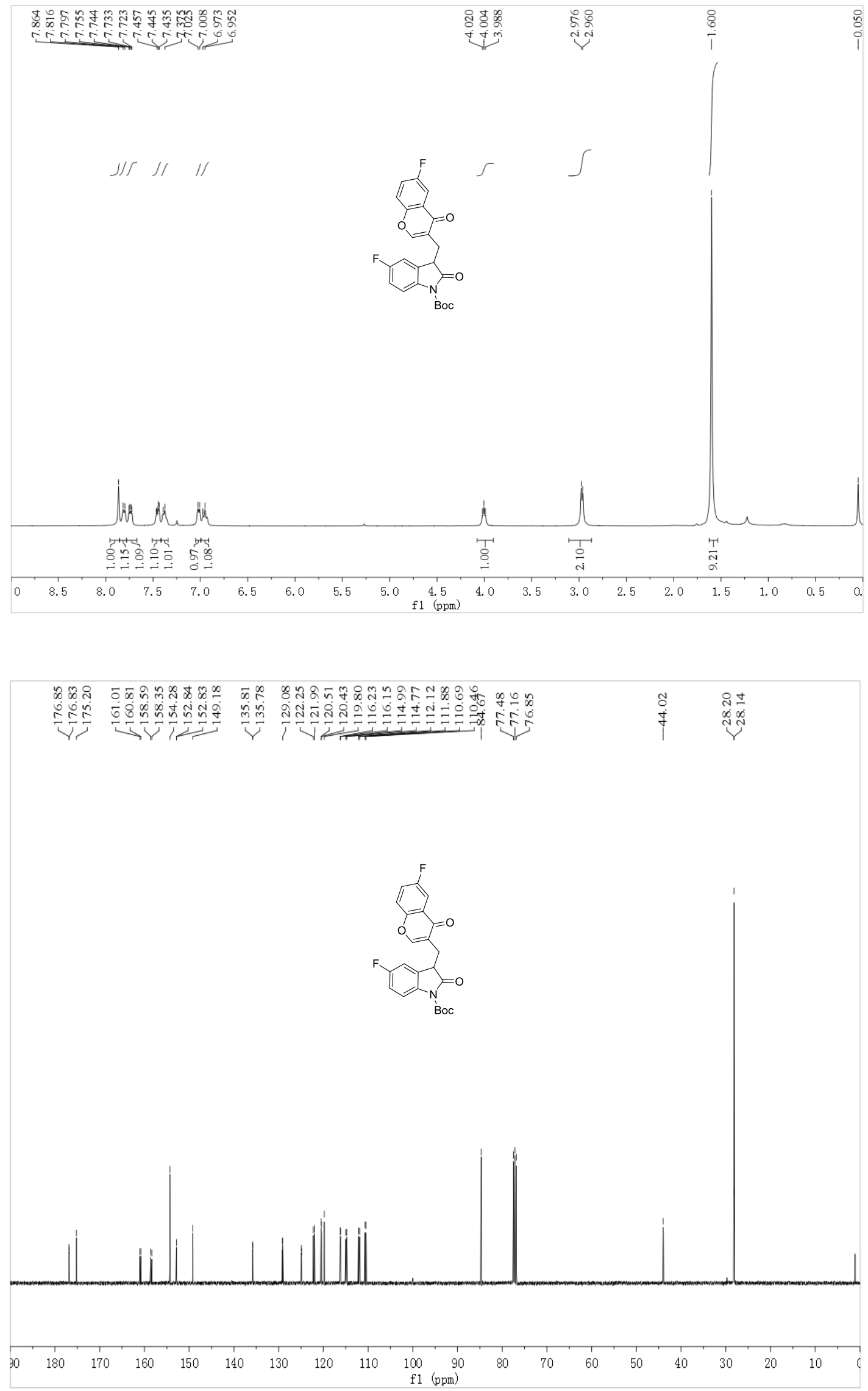
${ }^{19}$ F NMR of 1e

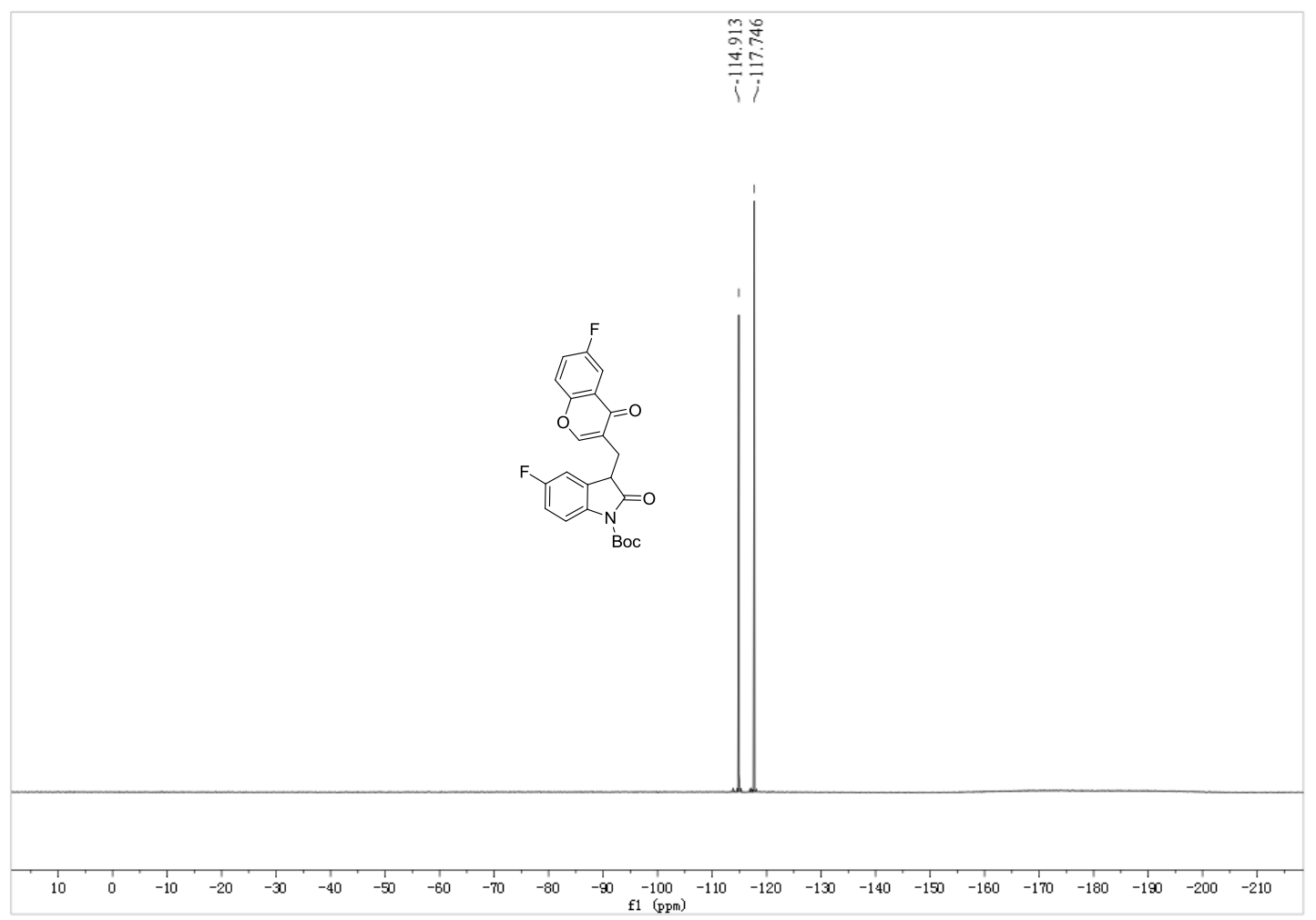


${ }^{1} \mathrm{H}$ and ${ }^{13} \mathrm{C}$ NMR of $1 f$
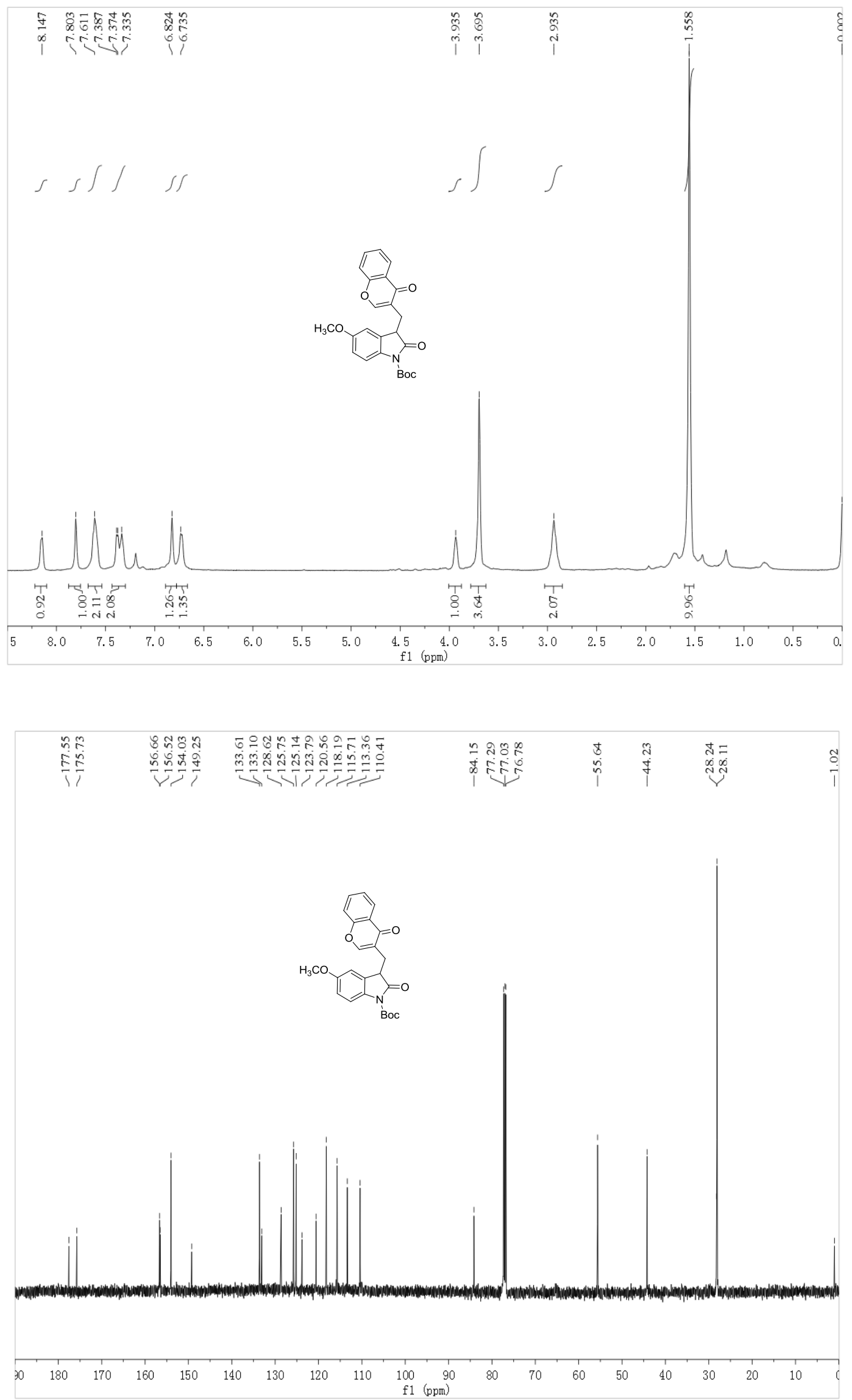


\section{${ }^{1} \mathrm{H}$ and ${ }^{13} \mathrm{C}$ NMR of $3^{\prime} \mathrm{a}$}
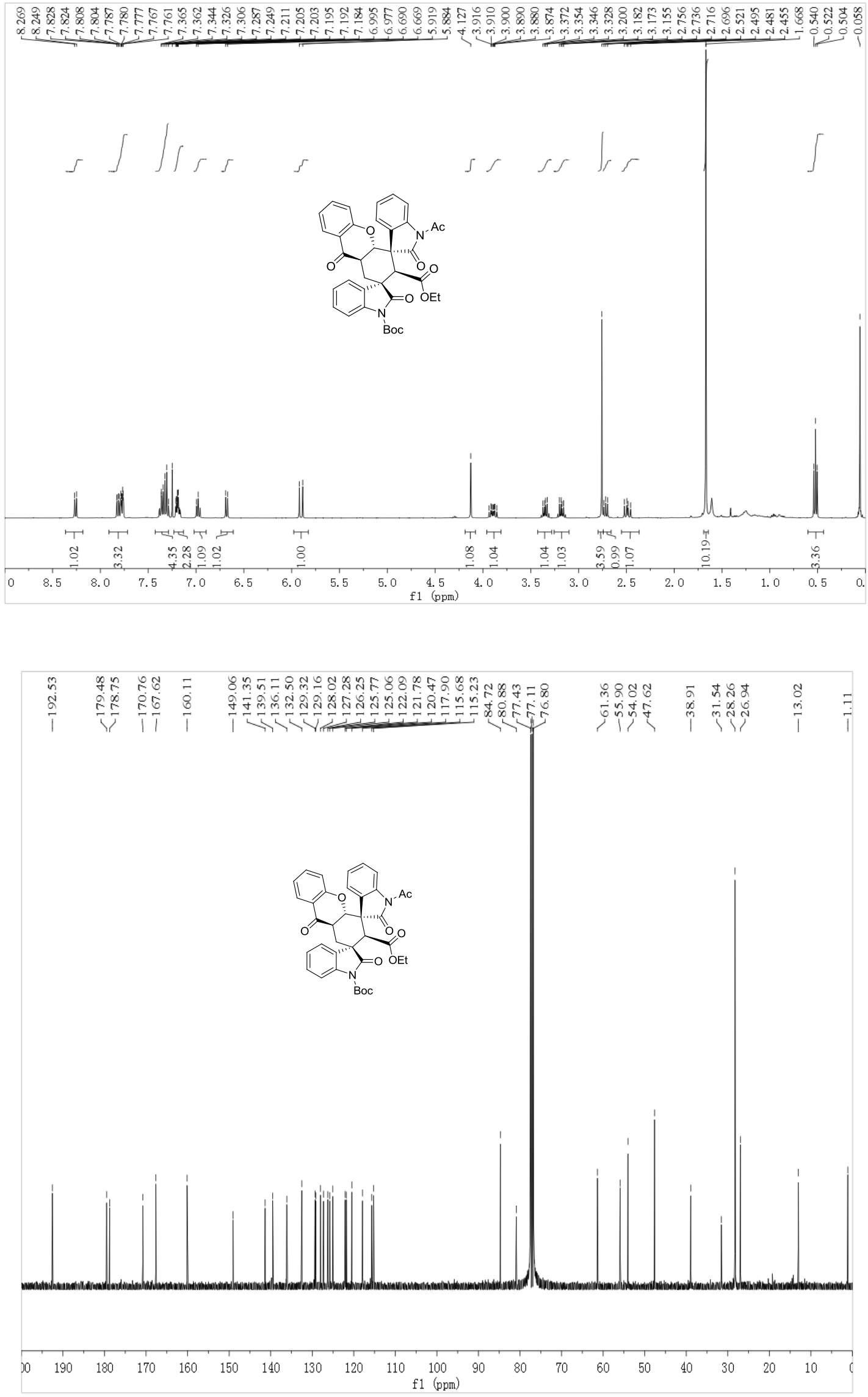
HPLC of 3'a

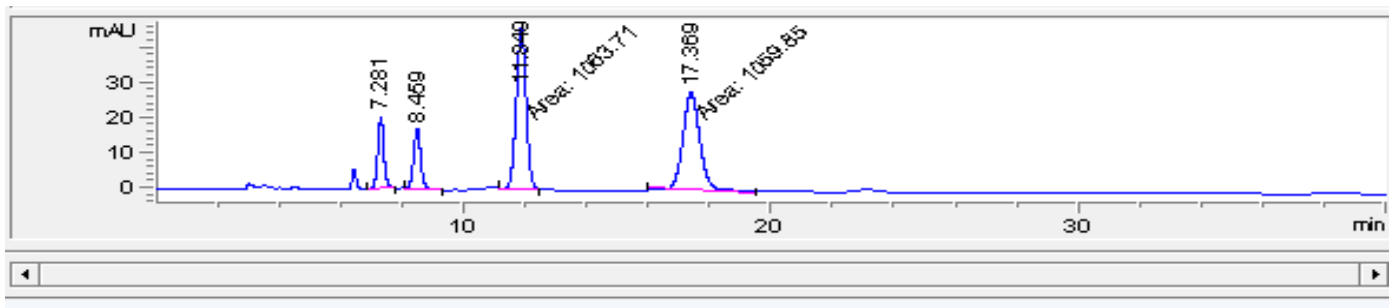

\begin{tabular}{|c|c|c|c|c|c|c|}
\hline$\#$ & Time & Area & Height & Width & Area\% & Symmetry \\
\hline 1 & 7.281 & 285.4 & 20.7 & 0.2087 & 10.611 & 1.003 \\
\hline 2 & 8.459 & 280.5 & 17.1 & 0.2509 & 10.428 & 0.933 \\
\hline 3 & 11.849 & 1063.7 & 46.2 & 0.3835 & 39.552 & 1.036 \\
\hline 4 & 17.369 & 1059.9 & 27.5 & 0.643 & 39.409 & 0.826 \\
\hline
\end{tabular}

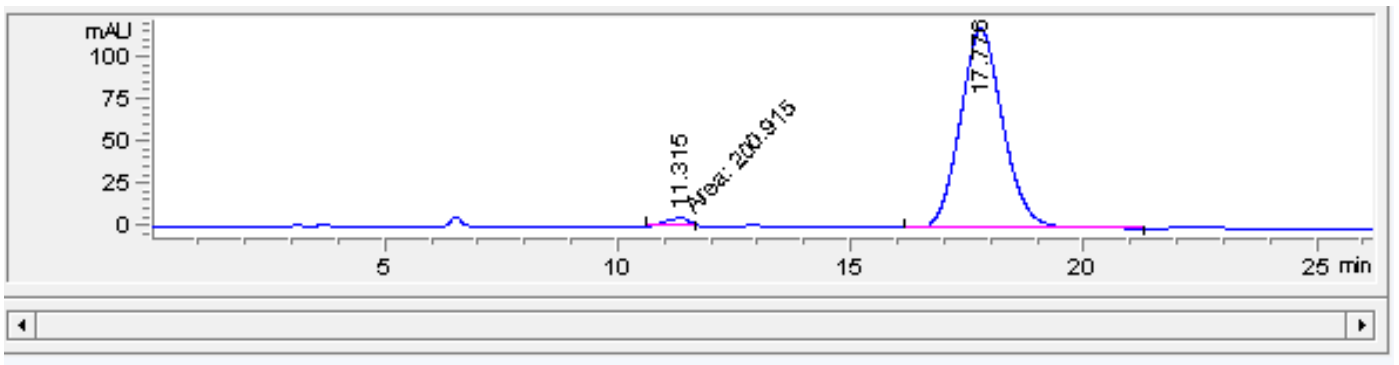

\begin{tabular}{|c|c|c|c|c|c|c|} 
\# & \multicolumn{1}{c}{ Time } & \multicolumn{1}{c}{ Area } & \multicolumn{1}{c}{ Height } & \multicolumn{1}{c}{ Width } & \multicolumn{2}{c}{ Area\% } \\
\hline 1 & 11.315 & 200.9 & 5.5 & 0.6072 & 2.635 & 1.624 \\
\hline 2 & 17.776 & 7424.4 & 118 & 0.9561 & 97.365 & 0.886 \\
\hline
\end{tabular}


${ }^{1} \mathrm{H}$ and ${ }^{13} \mathrm{C}$ NMR of $3 \mathrm{a}$

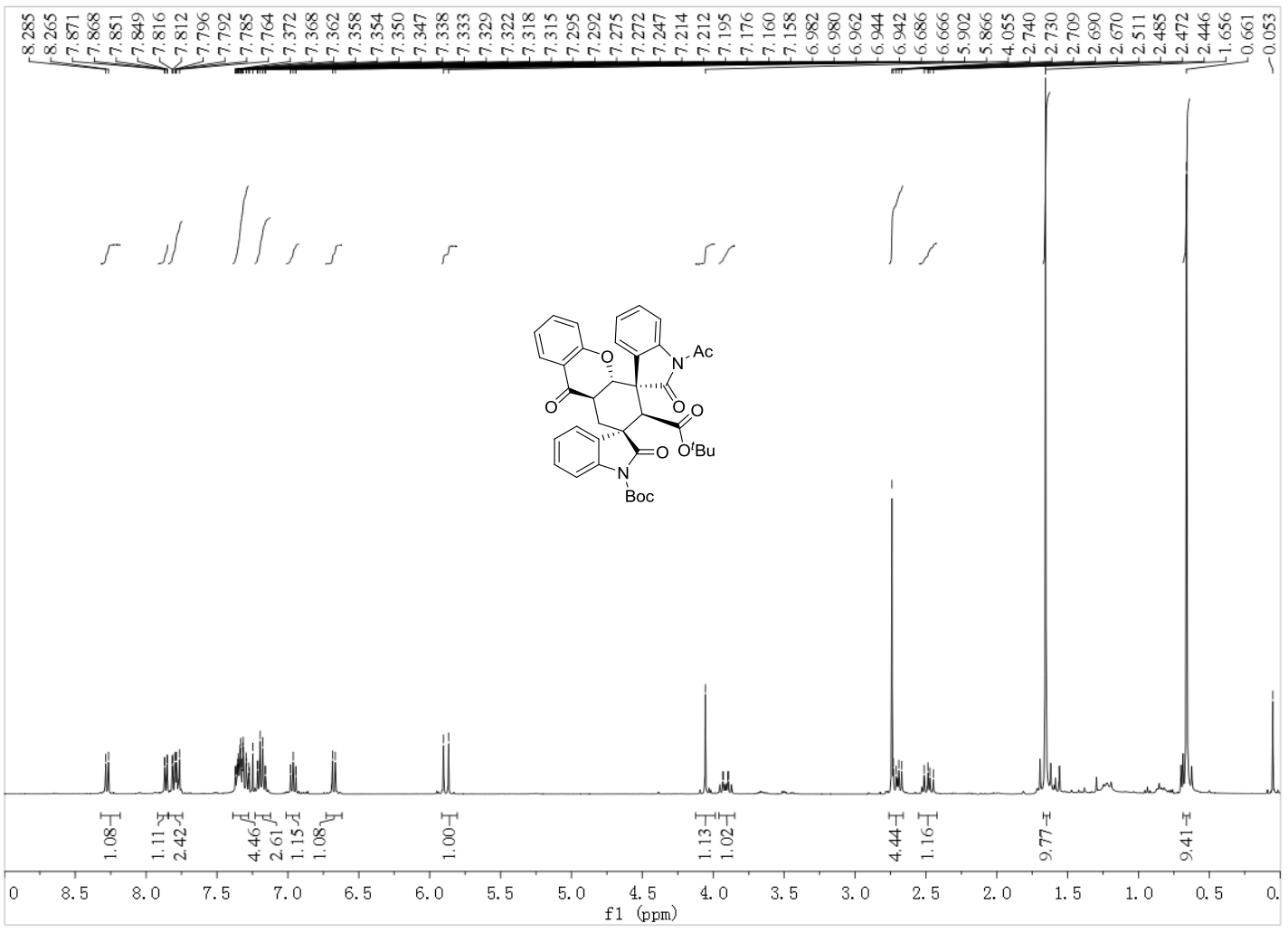

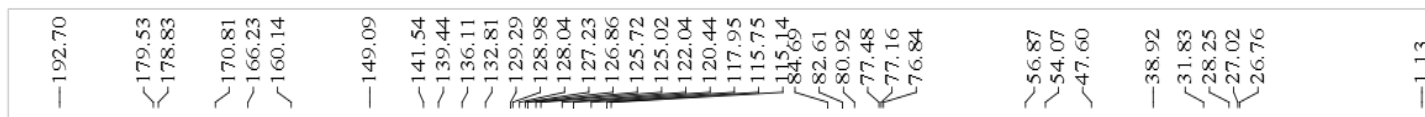
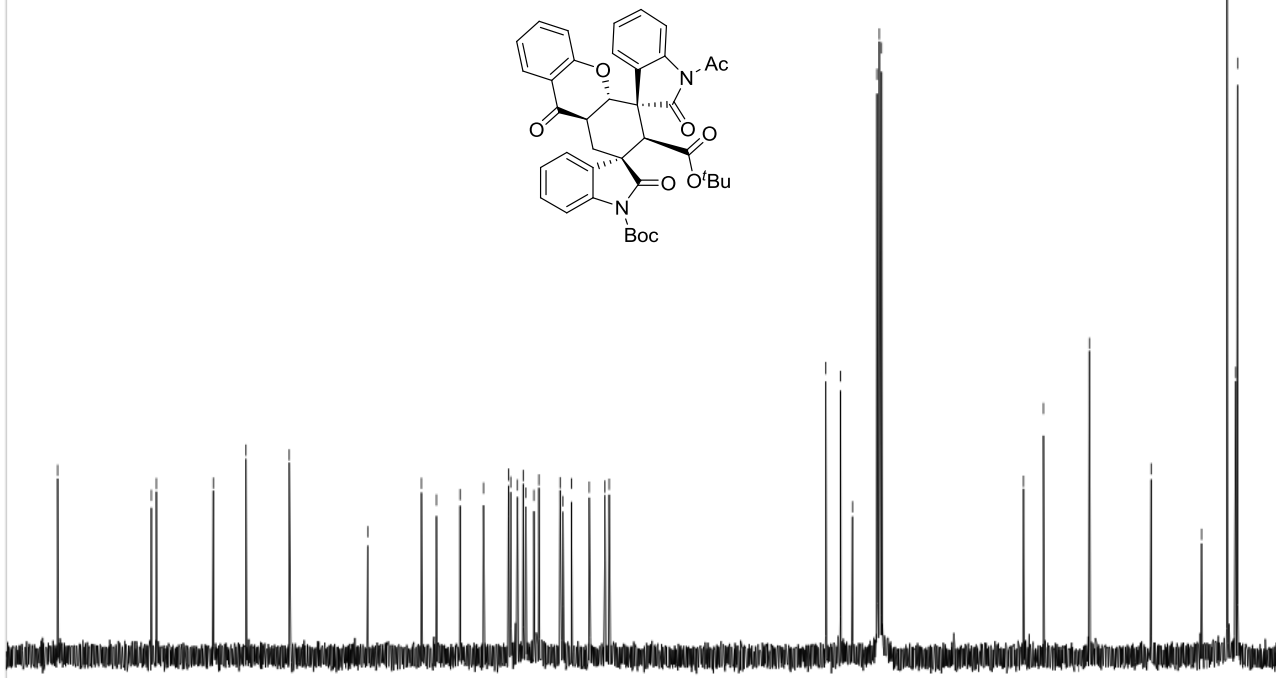

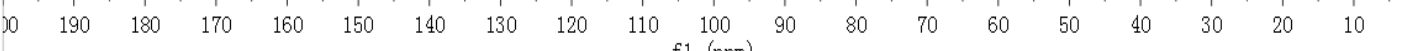


HPLC of 3a

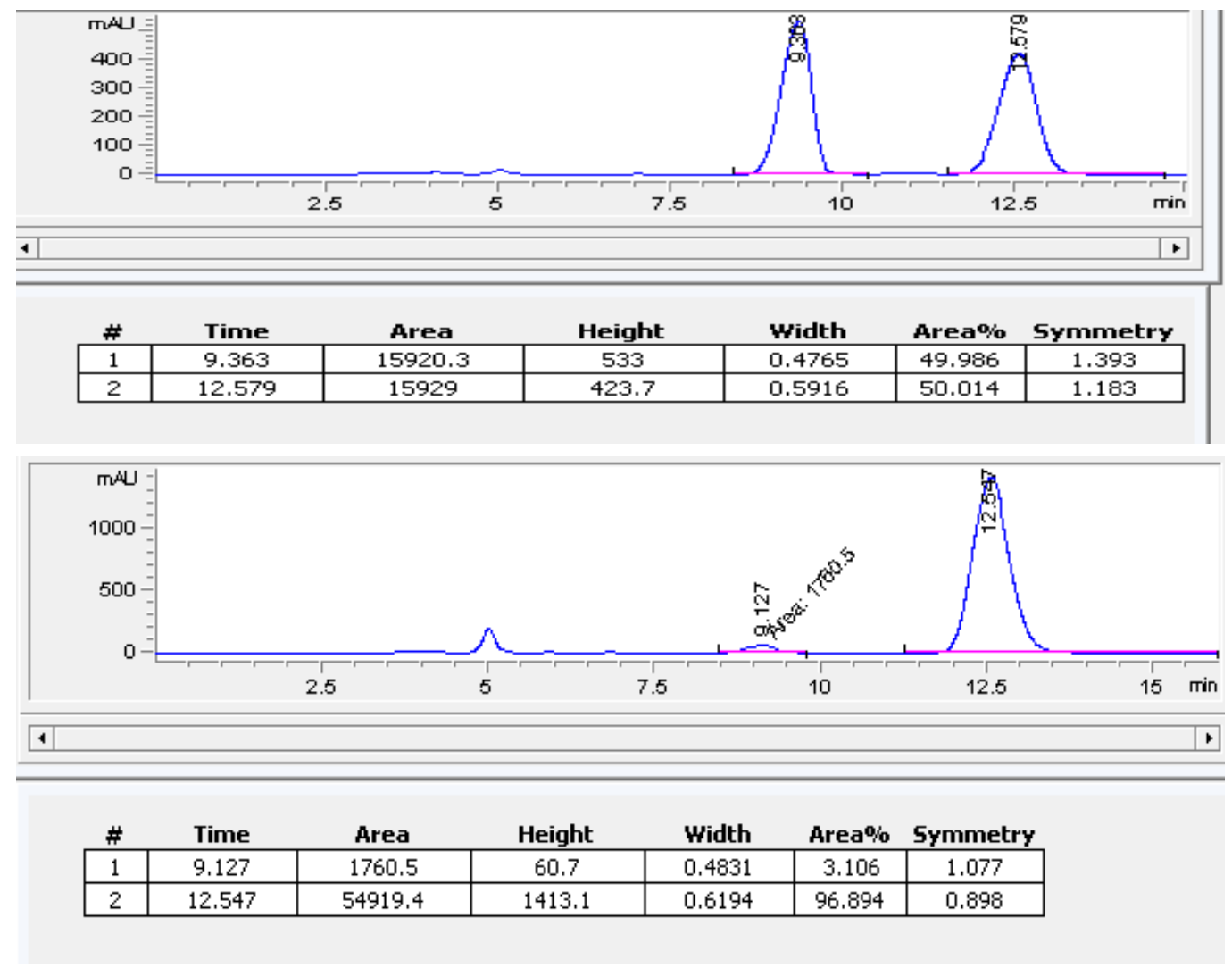


${ }^{1} \mathrm{H}$ and ${ }^{13} \mathrm{C}$ NMR of $3 \mathrm{~b}$

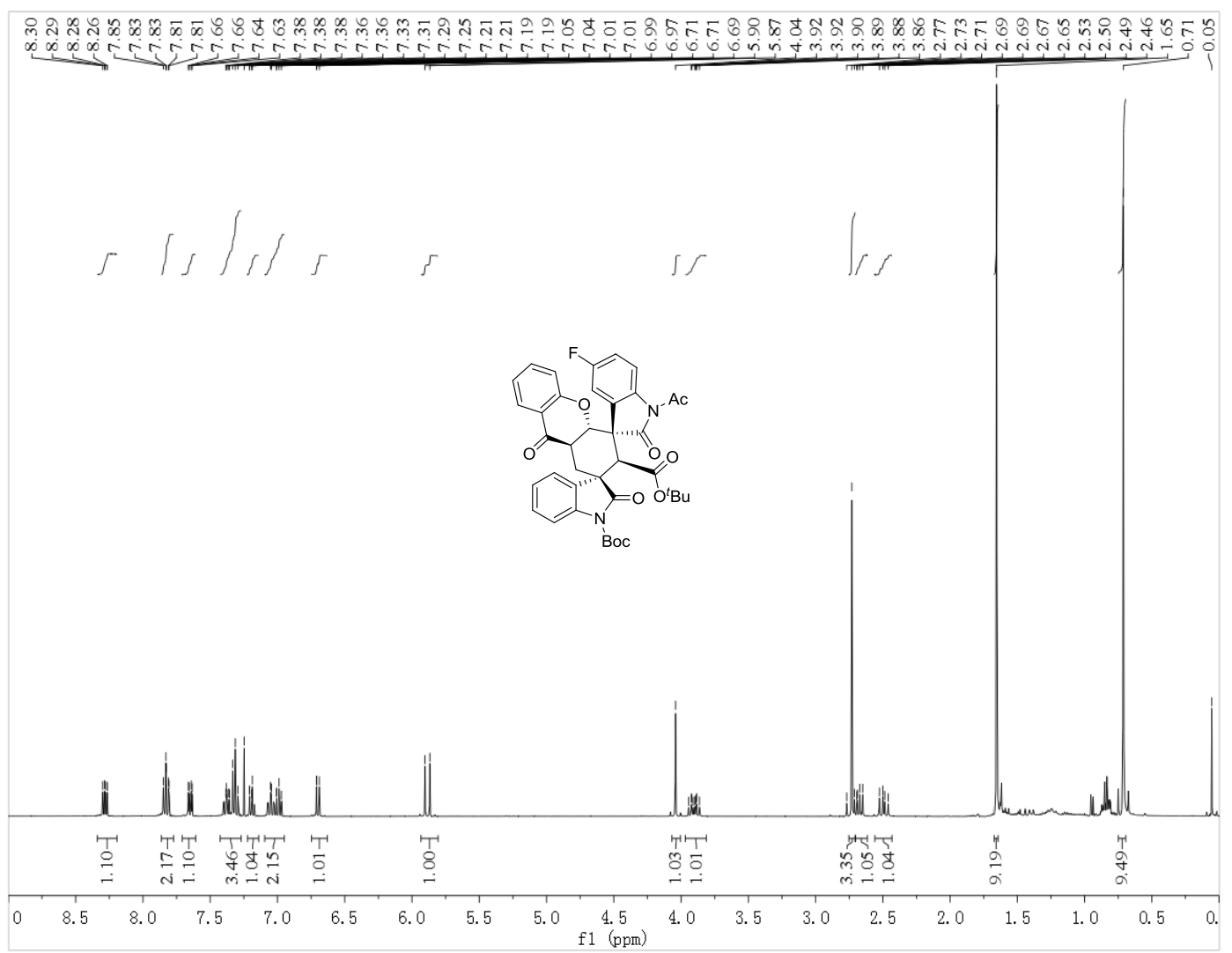

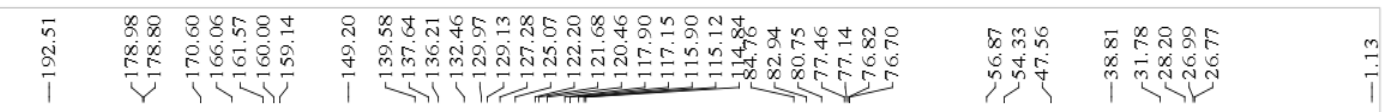

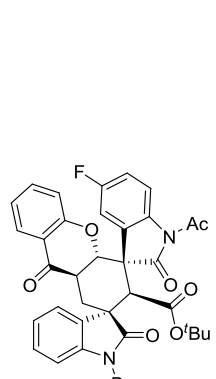

Boc

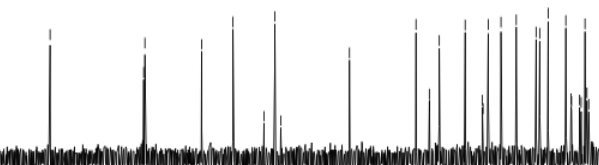

$110 \quad 100$ 
${ }^{19}$ F NMR of 3b

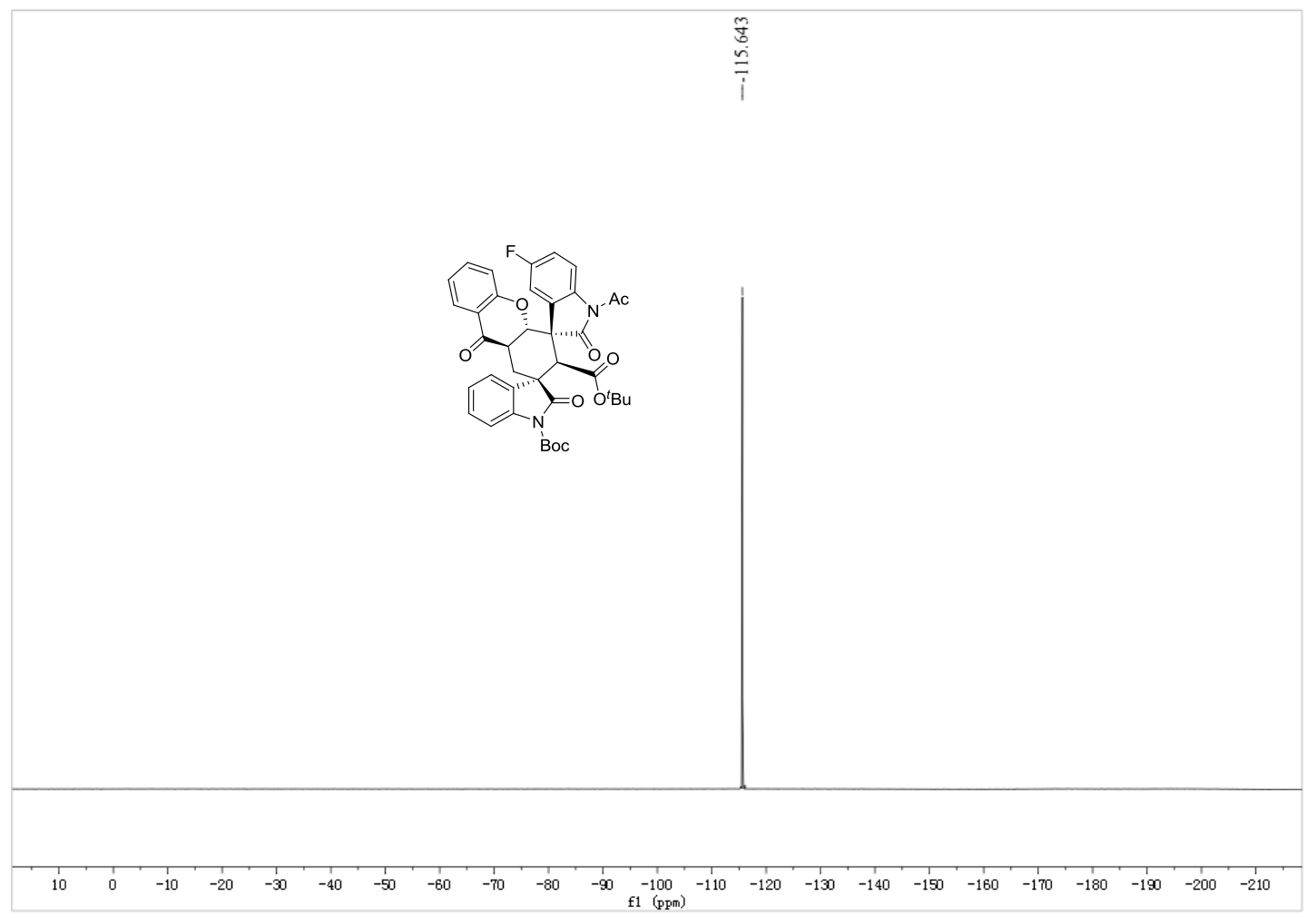

HPLC of 3b
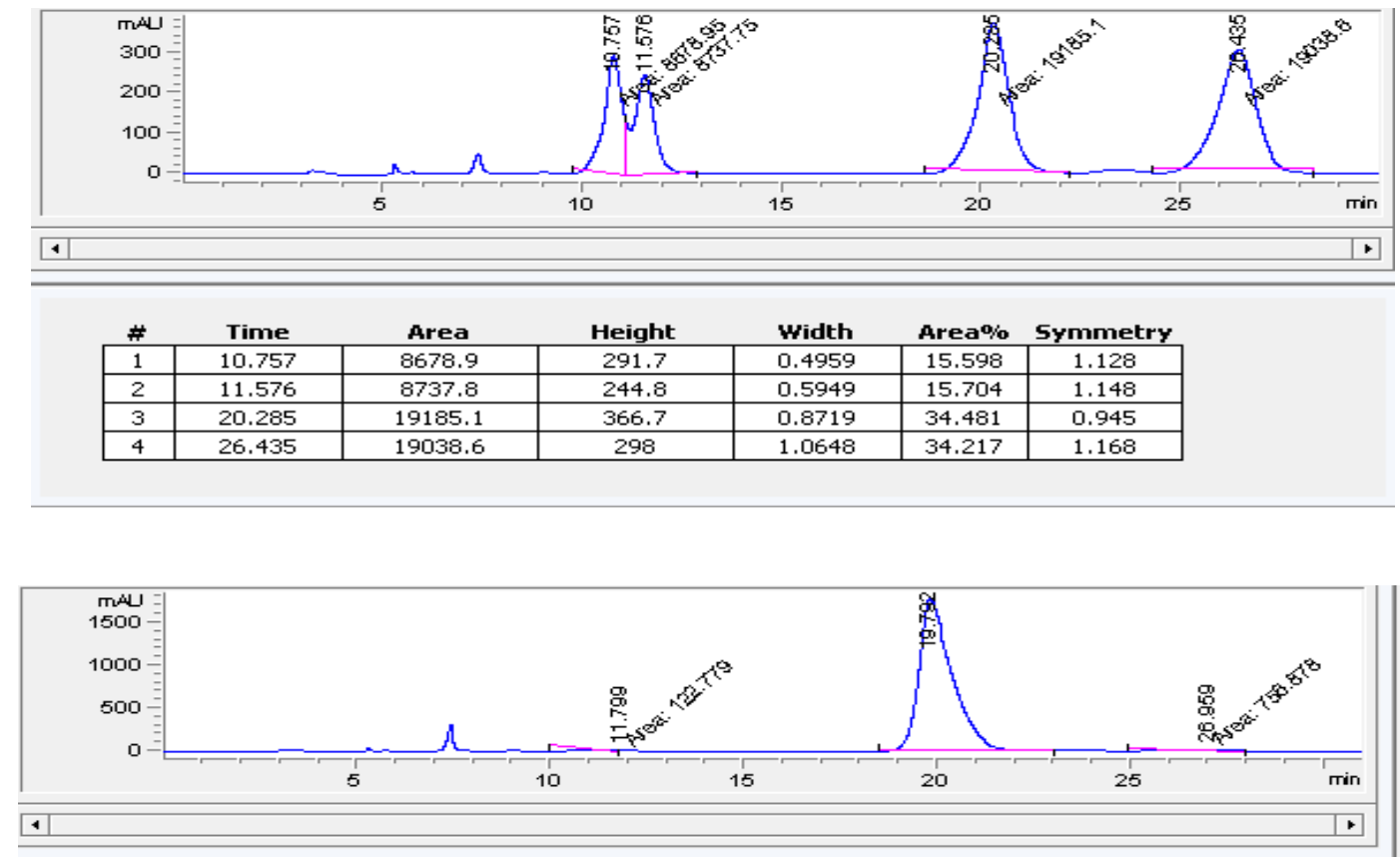

\begin{tabular}{|c|c|c|c|c|c|c|} 
\# & Time & \multicolumn{1}{c}{ Area } & Height & \multicolumn{1}{c}{ Width } & \multicolumn{2}{c}{ Area\% } \\
\hline 1 & 11.799 & 122.8 & 12.4 & 0.1646 & 0.116 & $1.23 \mathrm{E}-2$ \\
\hline 2 & 19.792 & 104902.1 & 1771.8 & 0.8428 & 99.168 & 0.462 \\
\hline 3 & 26.959 & 756.9 & 11.8 & 1.0687 & 0.716 & 0.235 \\
\hline
\end{tabular}


${ }^{1} \mathrm{H}$ and ${ }^{13} \mathrm{C}$ NMR of $3 \mathrm{c}$

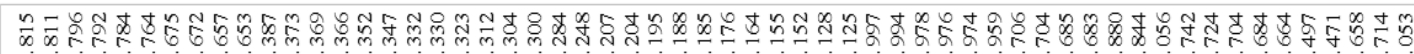

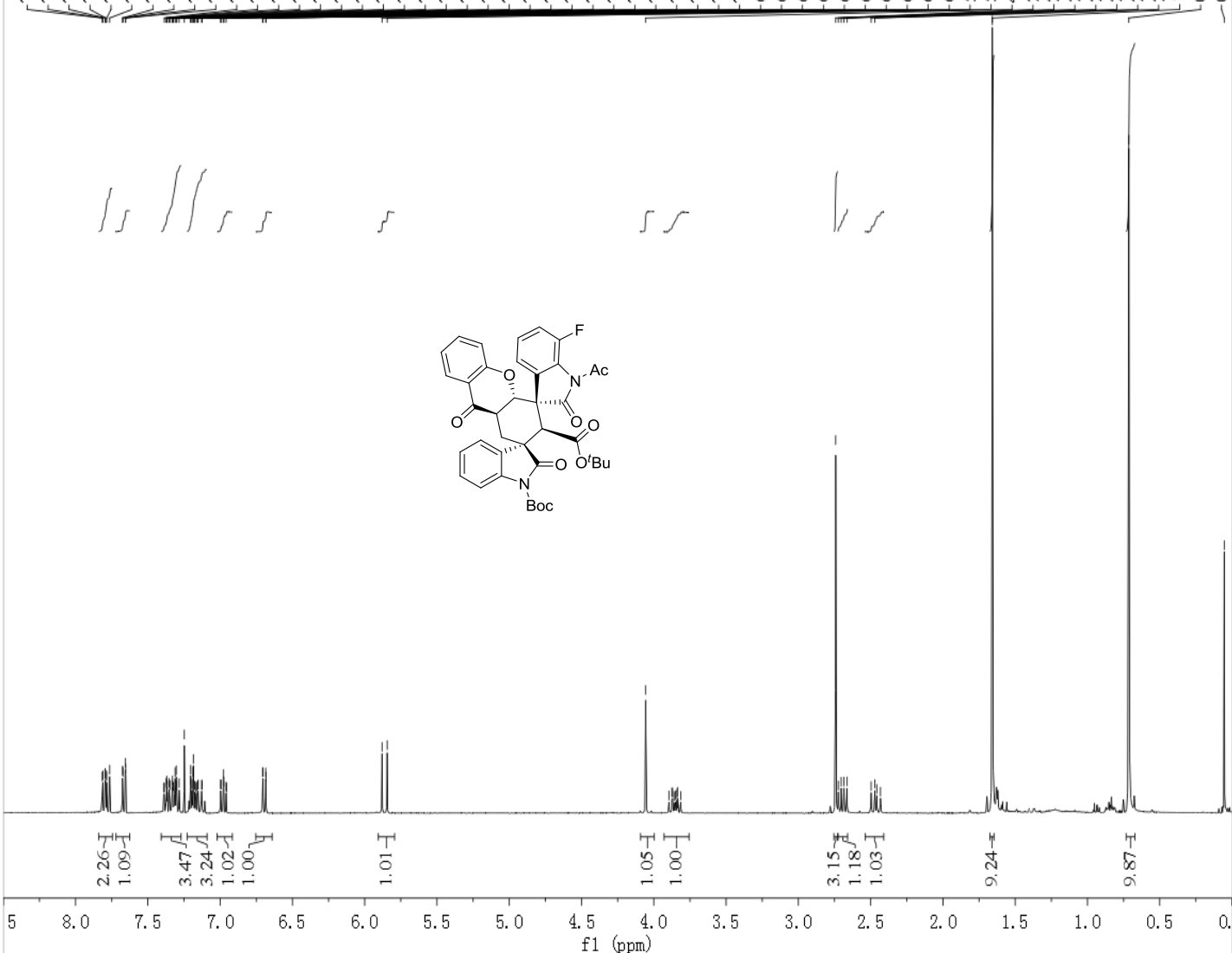

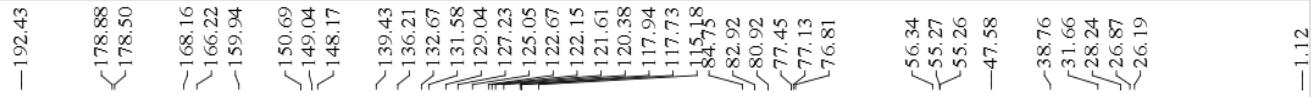

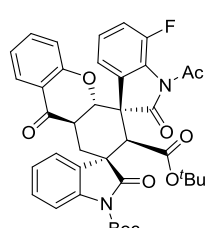

$\begin{array}{llllllllllll}10 & 200 & 190 & 180 & 170 & 160 & 150 & 140 & 130 & 120 & \begin{array}{c}110 \\ \mathrm{f} 1\end{array}(\mathrm{ppm})\end{array}$ 


\section{${ }^{19}$ F NMR of $3 c$}

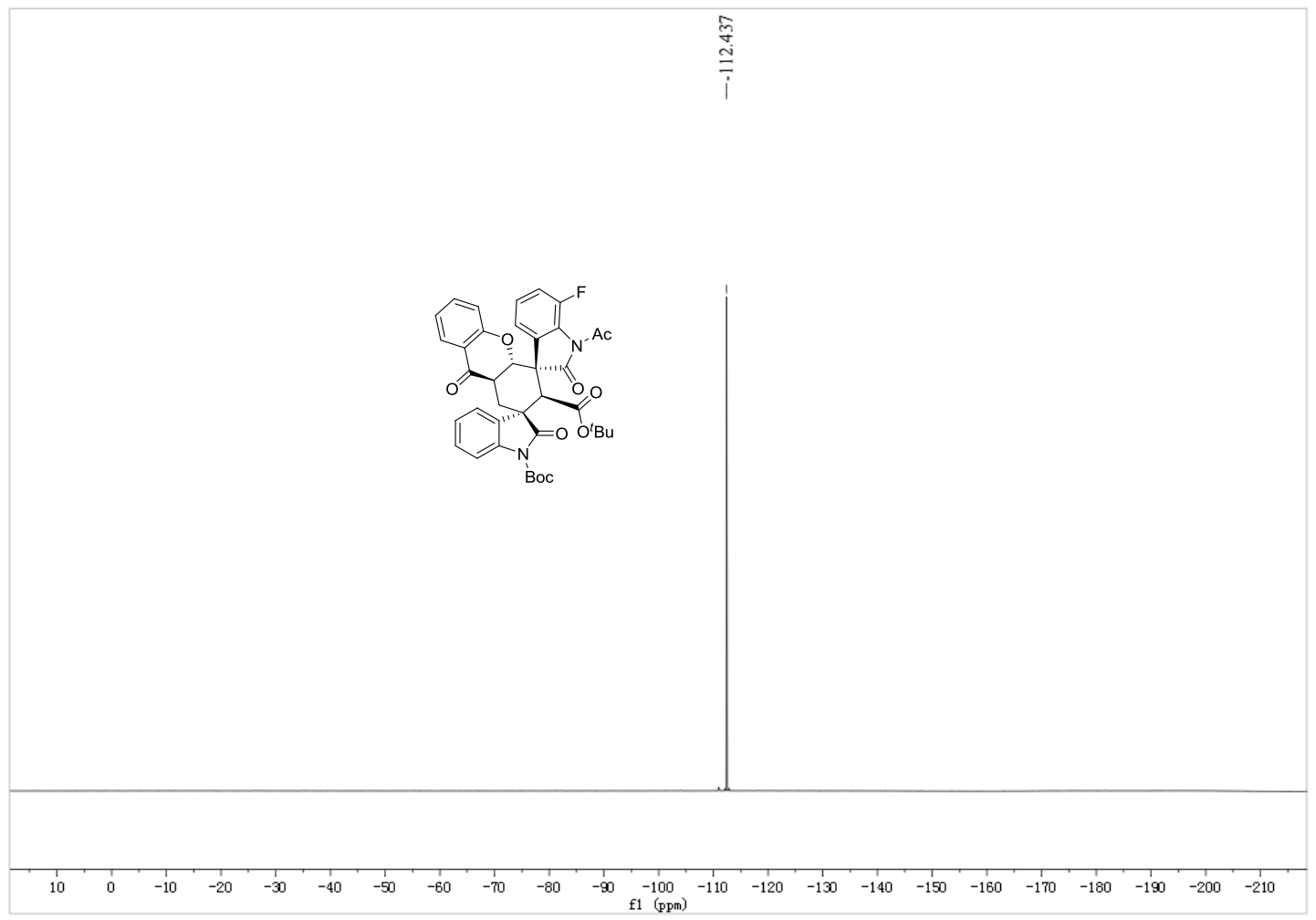

HPLC of 3c

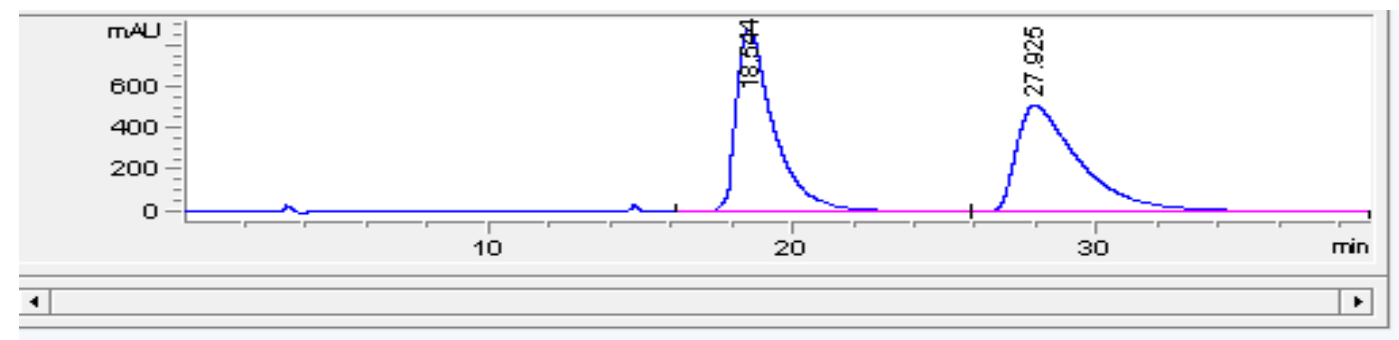

\begin{tabular}{|c|c|c|c|c|c|c|}
\hline$\#$ & Time & Area & Height & Width & Area $\%$ & Symmetry \\
\hline 1 & 18.544 & 76378.1 & 885 & 1.2429 & 49.928 & 0.477 \\
\hline 2 & 27.925 & 76598.4 & 515.5 & 2.1576 & 50.072 & 0.397 \\
\hline
\end{tabular}

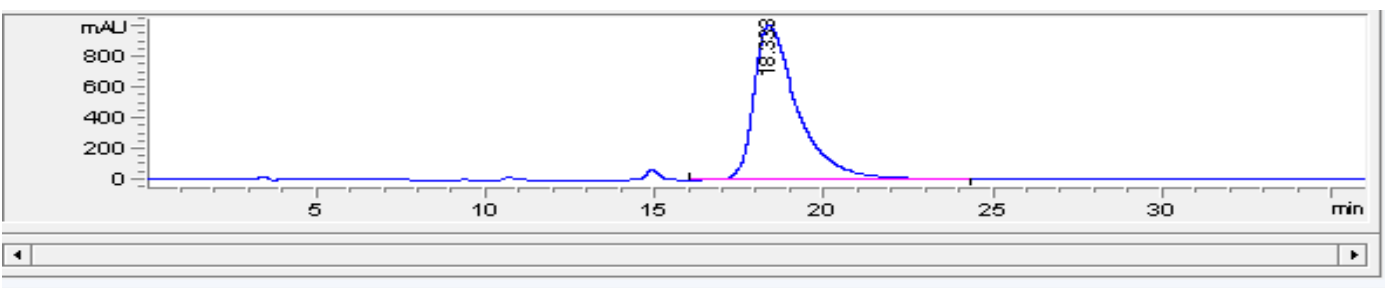

\begin{tabular}{|c|c|c|c|c|c|c|} 
\# & \multicolumn{1}{c}{ Time } & \multicolumn{1}{c}{ Area } & Height & Width & \multicolumn{2}{c}{ Area\% } \\
\hline 1 & 18.333 & 90491.2 & 1005.8 & 1.3217 & 100.000 & 0.466 \\
\hline
\end{tabular}


${ }^{1} \mathrm{H}$ and ${ }^{13} \mathrm{C}$ NMR of $3 d$

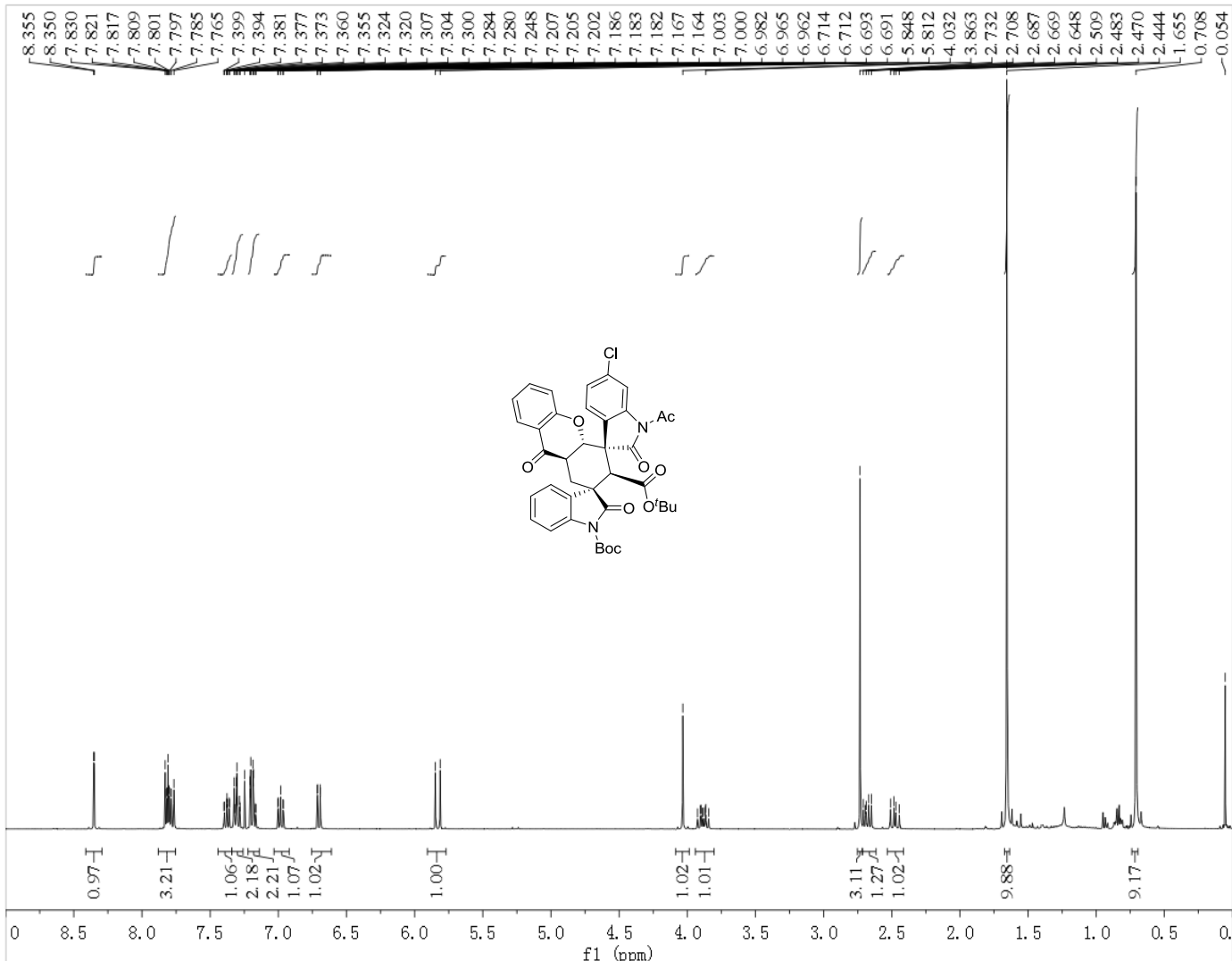

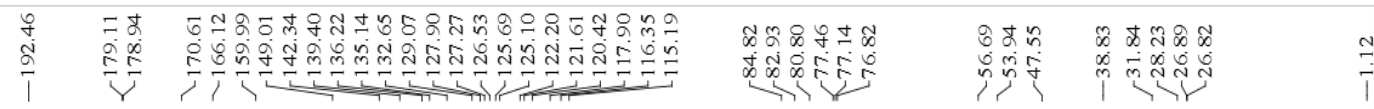

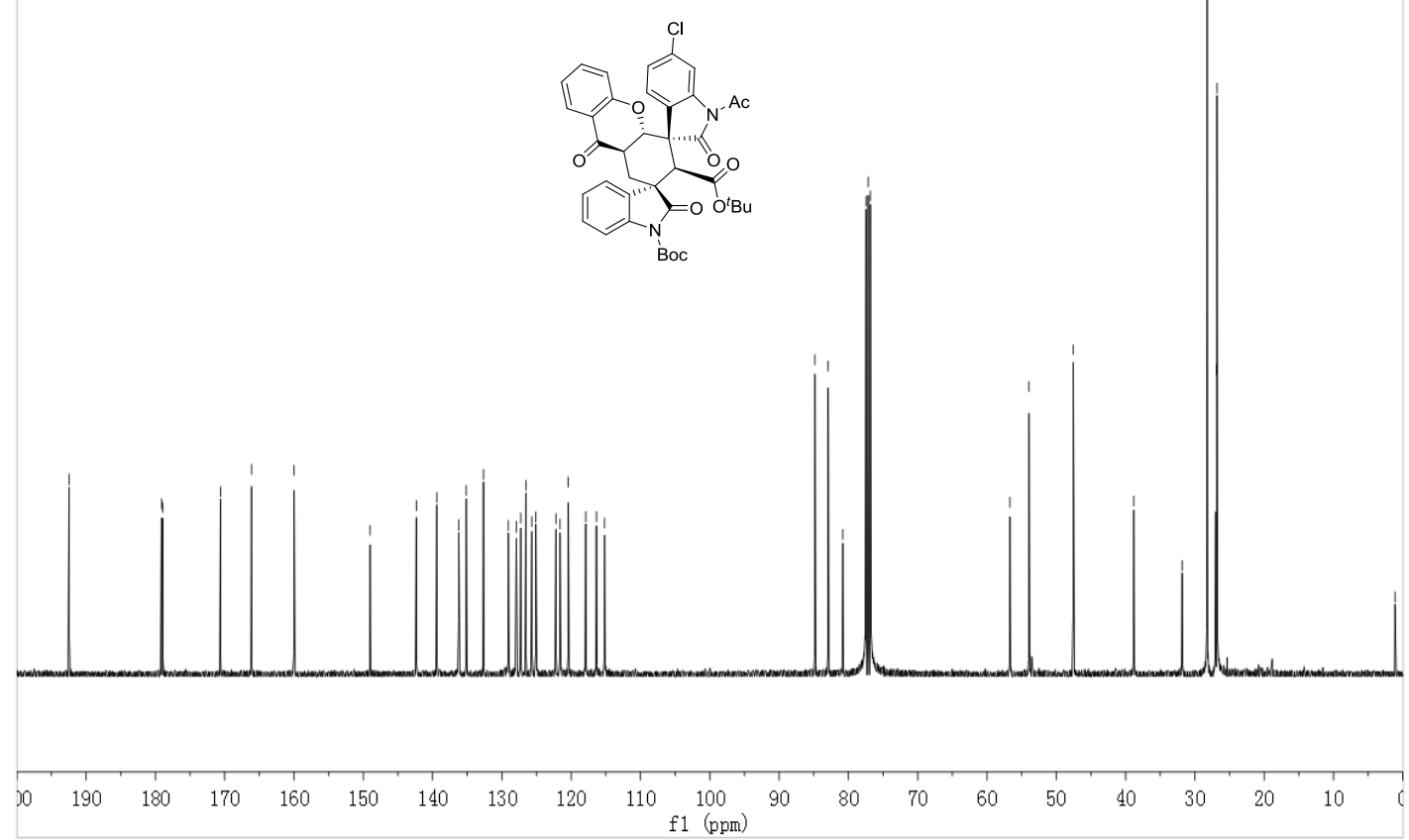


HPLC of 3d

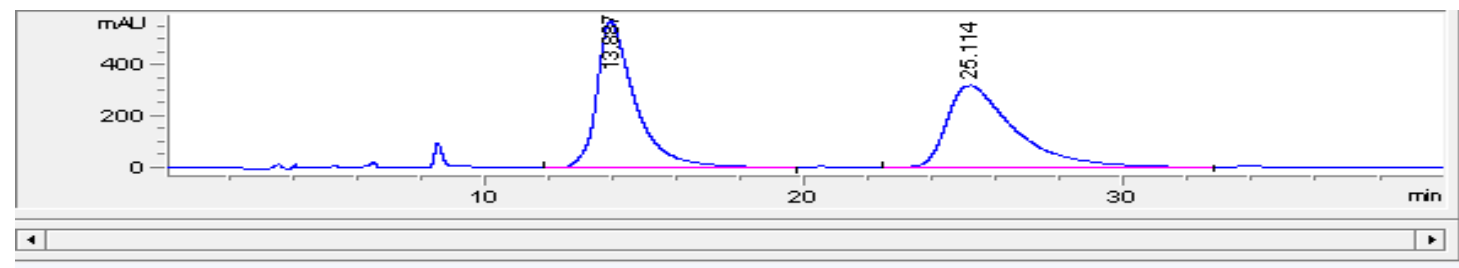

\begin{tabular}{|c|c|c|c|c|c|c|}
\multicolumn{2}{l}{ Time } & \multicolumn{1}{c}{ Area } & Height & \multicolumn{1}{c}{ Width } & \multicolumn{1}{c}{ Area\% } & Symmetry \\
\hline 1 & 13.887 & 47970.6 & 569 & 1.2277 & 49.951 & 0.513 \\
\hline 2 & 25.114 & 48064.1 & 320.6 & 2.2229 & 50.049 & 0.46 \\
\hline
\end{tabular}

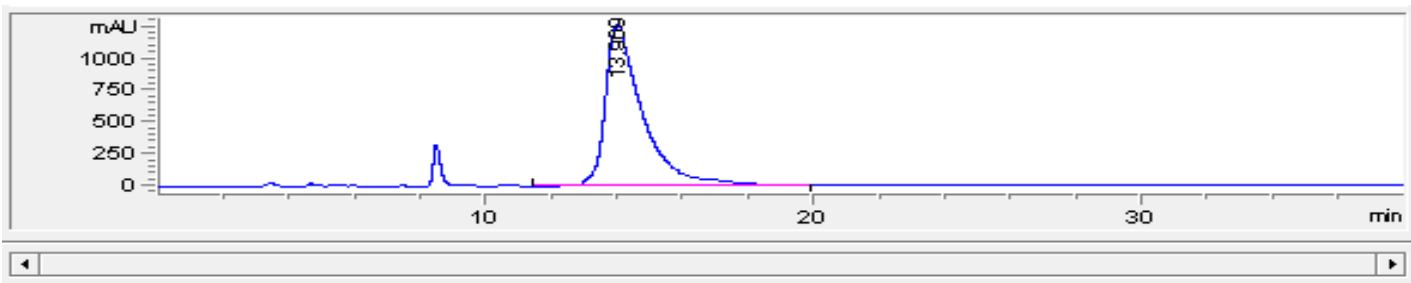

\begin{tabular}{|c|c|c|c|c|c|c|} 
\# & \multicolumn{1}{c}{ Time } & \multicolumn{1}{c}{ Area } & Height & Width & \multicolumn{1}{c}{ Area\% $\%$ Symmetry } \\
\hline 1 & 13.969 & 102269.7 & 1263.6 & 1.1842 & 100.000 & 0.425 \\
\hline
\end{tabular}


${ }^{1} \mathrm{H}$ and ${ }^{13} \mathrm{C}$ NMR of $3 e$
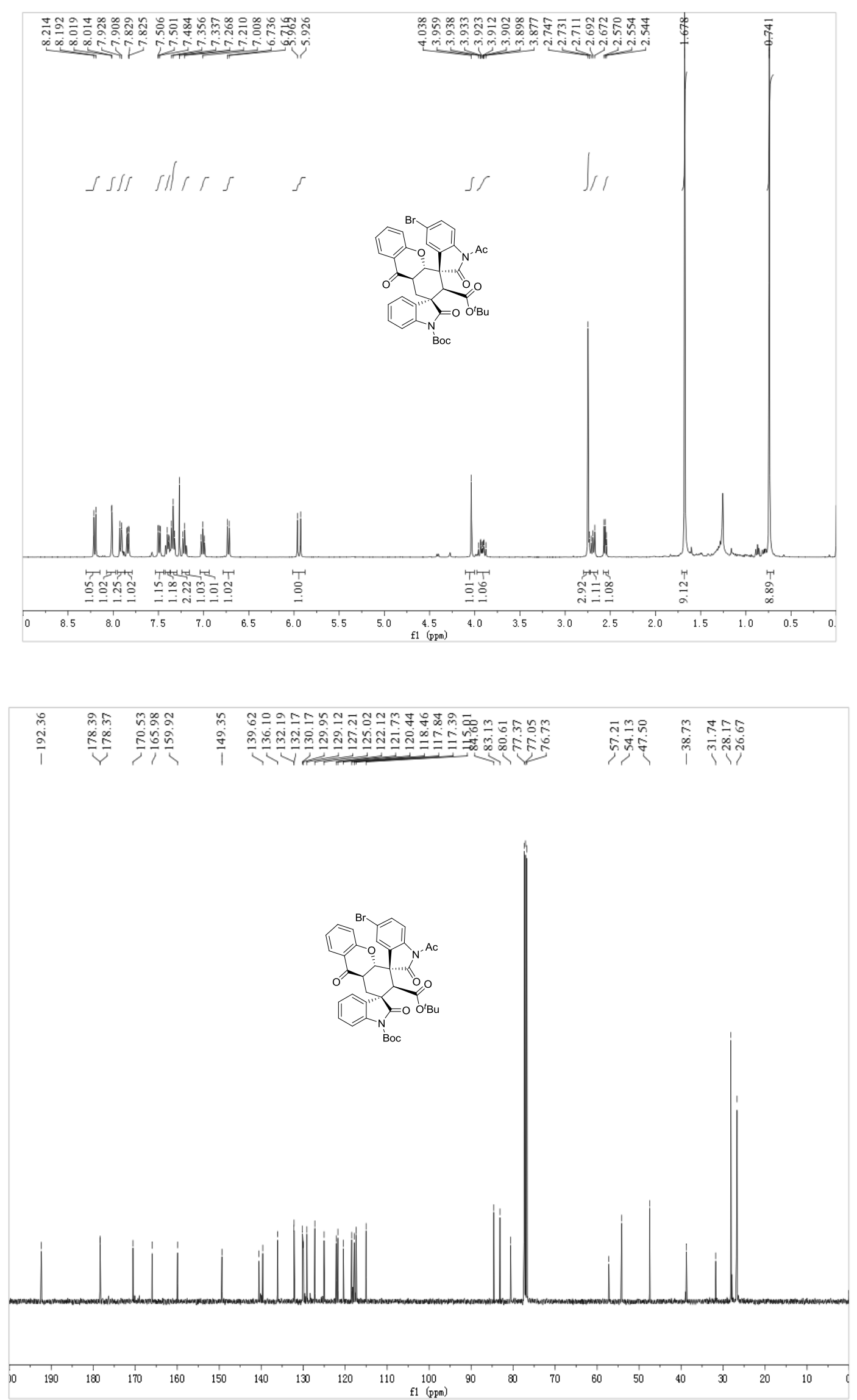
HPLC of 3e

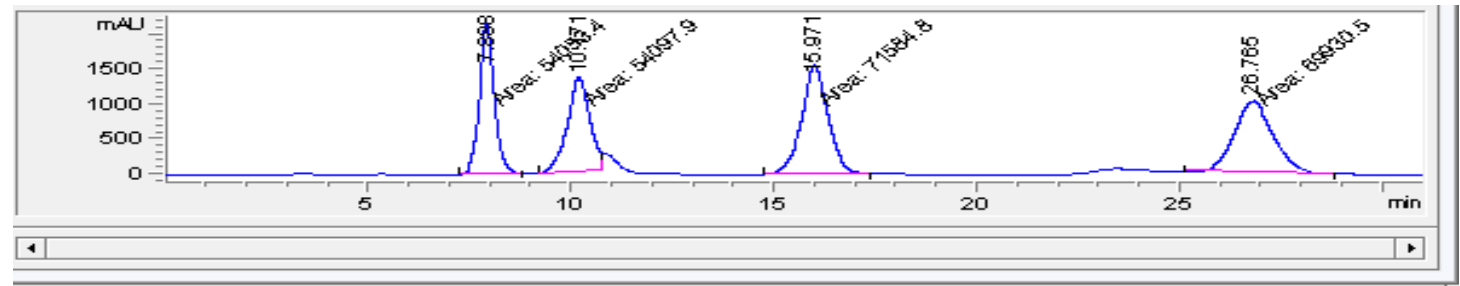

\begin{tabular}{|c|c|c|c|c|c|c|}
\hline \# & Time & Area & Height & Width & Area\% & Symmetry \\
\hline 1 & 7.888 & 54053.4 & 2184 & 0.4125 & 21.650 & 0.816 \\
\hline 2 & 10.171 & 54097.9 & 1366 & 0.6601 & 21.668 & 1.042 \\
\hline 3 & 15.971 & 71584.8 & 1573.5 & 0.7582 & 28.672 & 0.91 \\
\hline 4 & 26.765 & 69930.5 & 1033.9 & 1.1273 & 28.010 & 0.816 \\
\hline
\end{tabular}

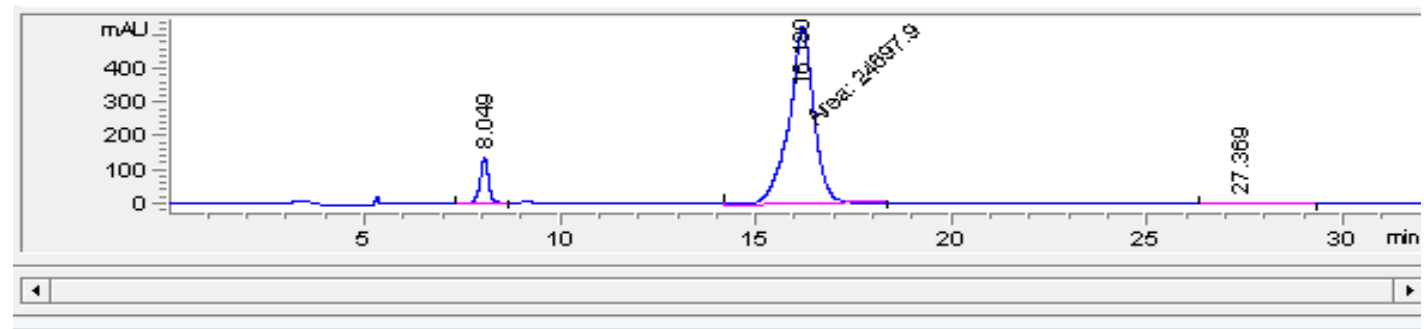

\begin{tabular}{|c|c|c|c|c|c|c|} 
\# & \multicolumn{1}{c}{ Time } & \multicolumn{1}{c}{ Area } & Height & \multicolumn{1}{c|}{ Width } & \multicolumn{2}{c|}{ Area\% } \\
\hline 1 & 8.049 & 2065.4 & 137.7 & 0.2229 & 7.643 & 1.062 \\
\hline 2 & 16.18 & 24697.9 & 531.8 & 0.7741 & 91.399 & 1.21 \\
\hline 3 & 27.369 & 258.8 & 3.5 & 1.0988 & 0.958 & 1.168 \\
\hline
\end{tabular}


${ }^{1} \mathrm{H}$ and ${ }^{13} \mathrm{C}$ NMR of $3 f$
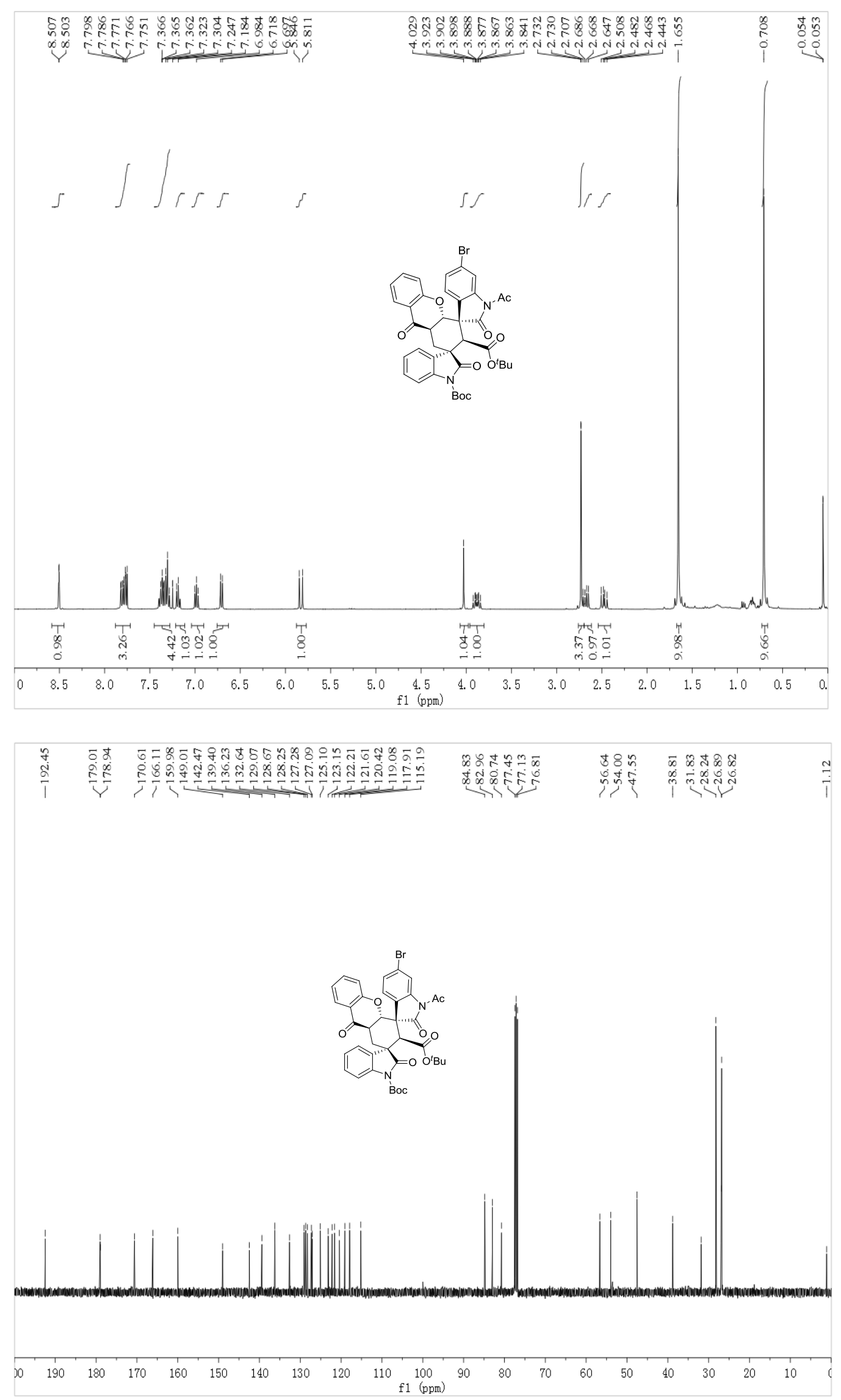
HPLC of $3 f$
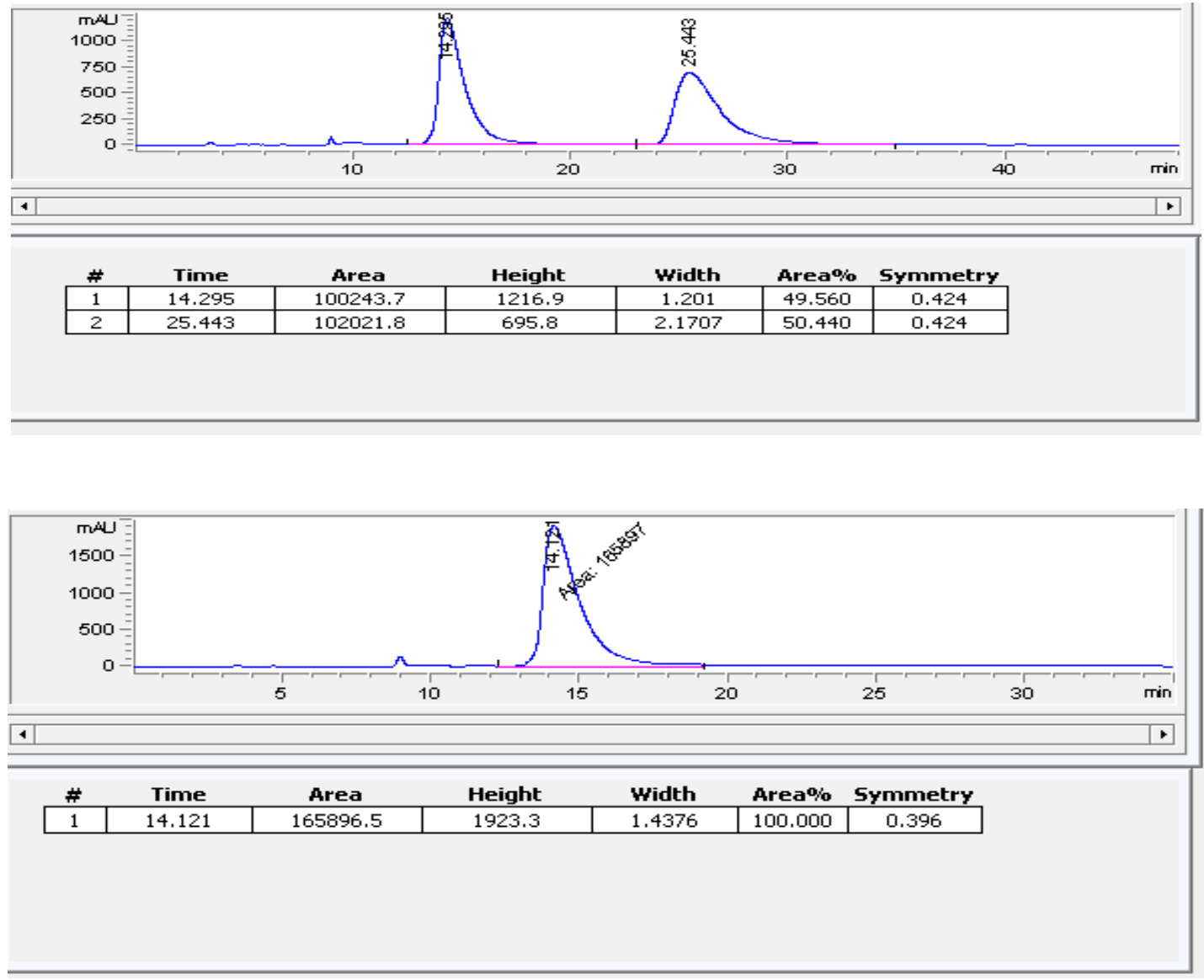


\section{${ }^{1} \mathrm{H}$ and ${ }^{13} \mathrm{C}$ NMR of $3 \mathrm{~g}$}
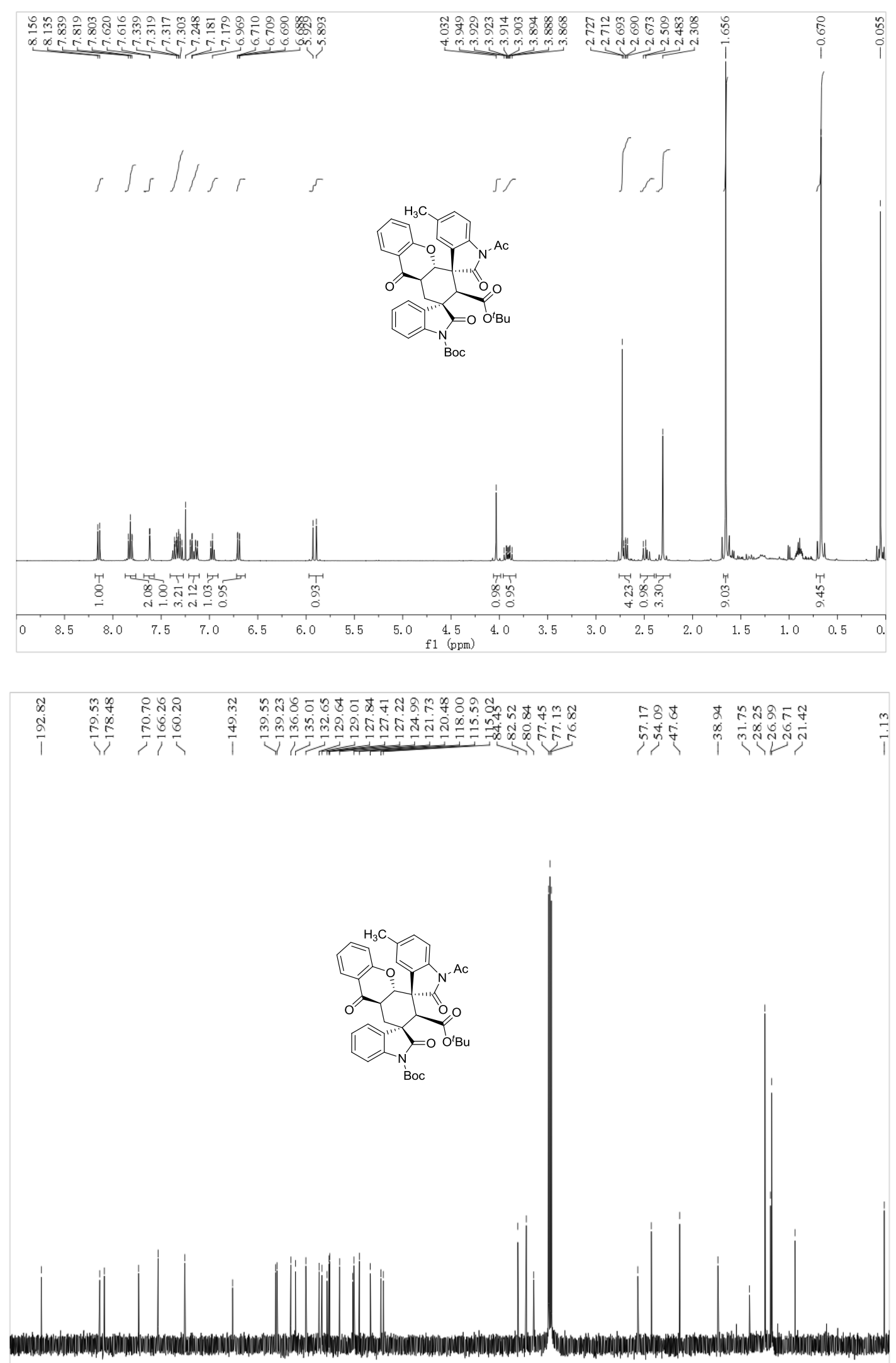

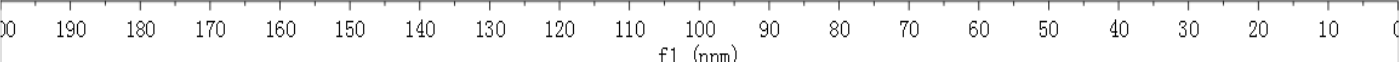


HPLC of $3 g$

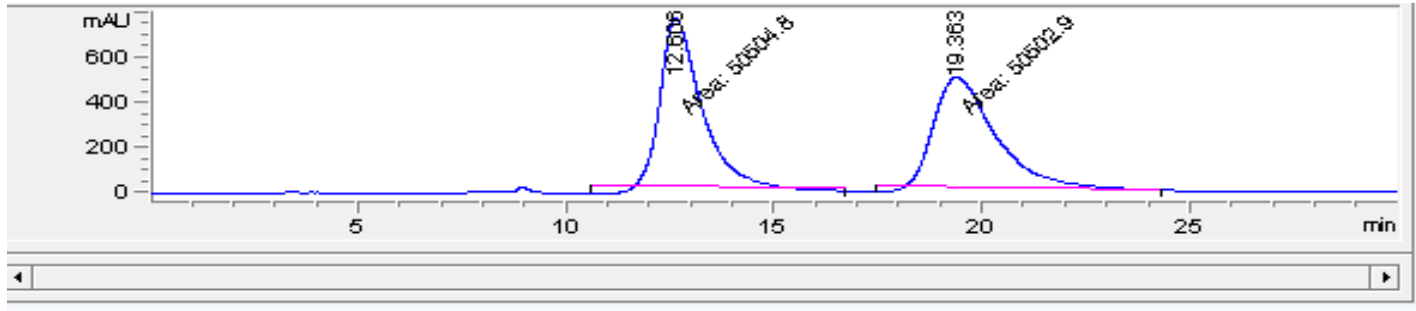

\begin{tabular}{|c|c|c|c|c|c|c|} 
\# & \multicolumn{1}{c}{ Time } & \multicolumn{1}{c}{ Area } & Height & Width & \multicolumn{1}{c}{ Area\% $\%$} & Symmetry \\
\hline 1 & 12.606 & 50504.8 & 746.5 & 1.1275 & 50.001 & 0.577 \\
\hline 2 & 19.363 & 50502.9 & 486.2 & 1.731 & 49.999 & 0.535 \\
\hline
\end{tabular}

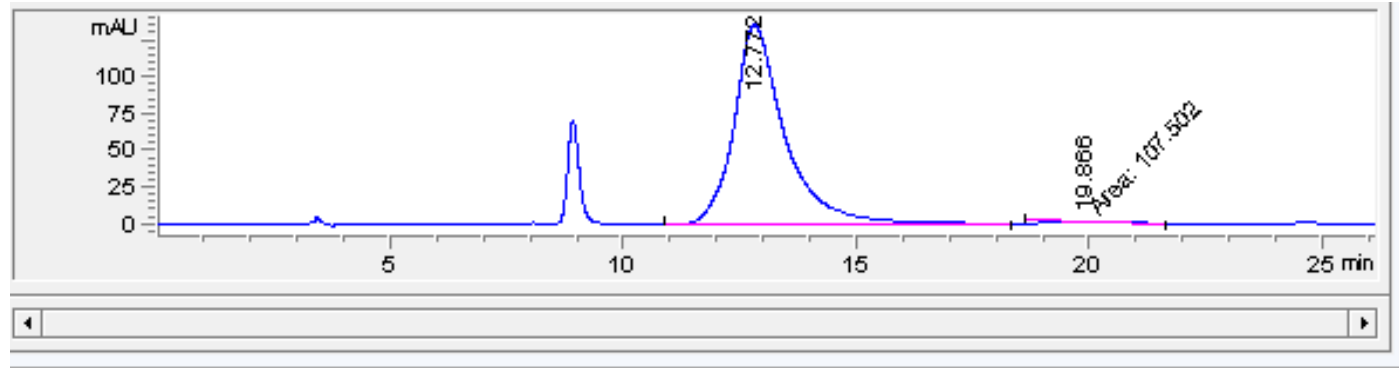

\begin{tabular}{|c|c|c|c|c|c|c|}
\hline$\#$ & Time & Area & Height & Width & Area $\%$ & Symmetry \\
\hline 1 & 12.772 & 10408.2 & 135.7 & 1.1052 & 98.978 & 0.62 \\
\hline 2 & 19.866 & 107.5 & $9.9 \mathrm{E}-1$ & 1.8044 & 1.022 & 0.242 \\
\hline
\end{tabular}


${ }^{1} \mathrm{H}$ and ${ }^{13} \mathrm{C}$ NMR of $3 \mathrm{~h}$
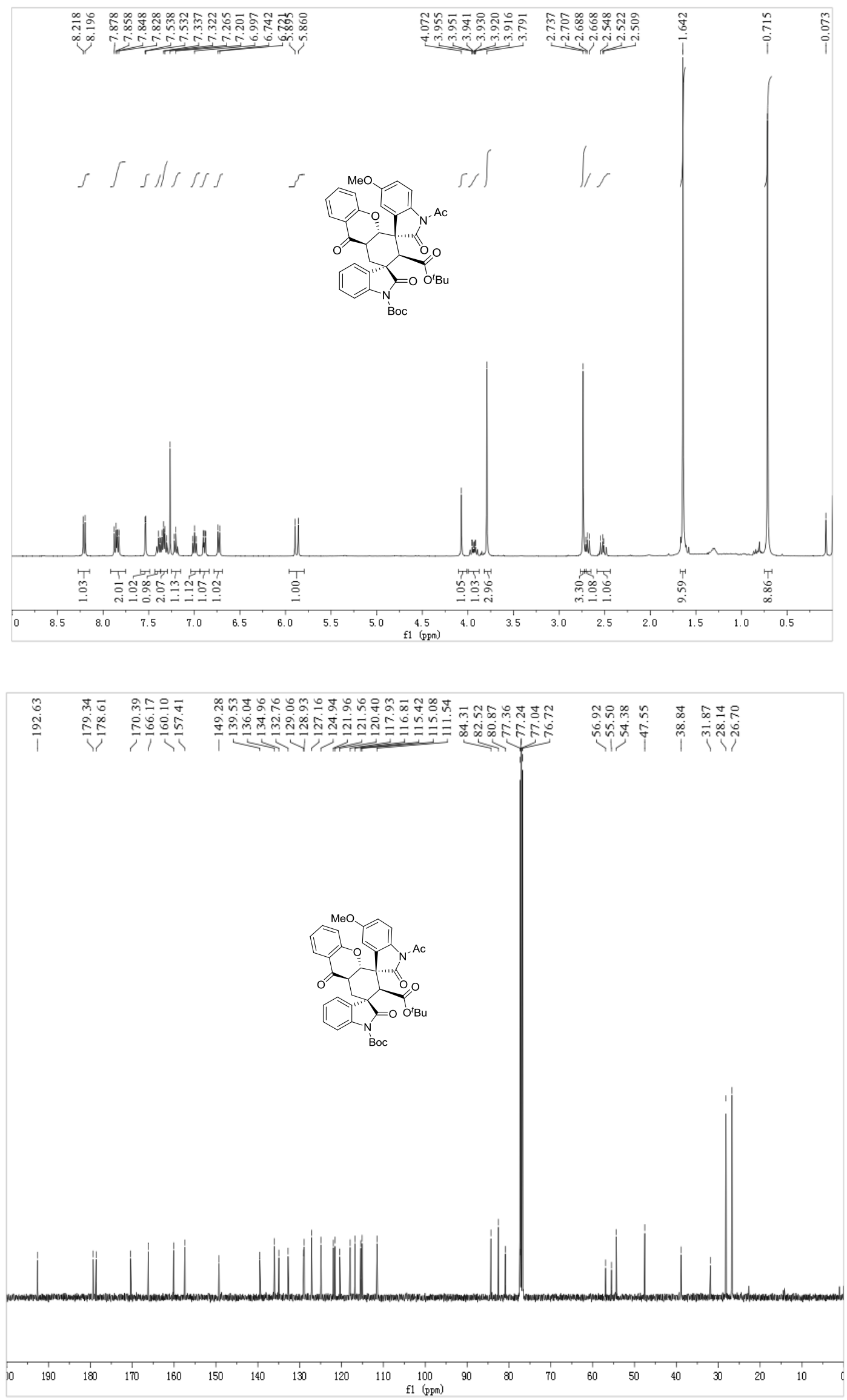
HPLC of $3 h$

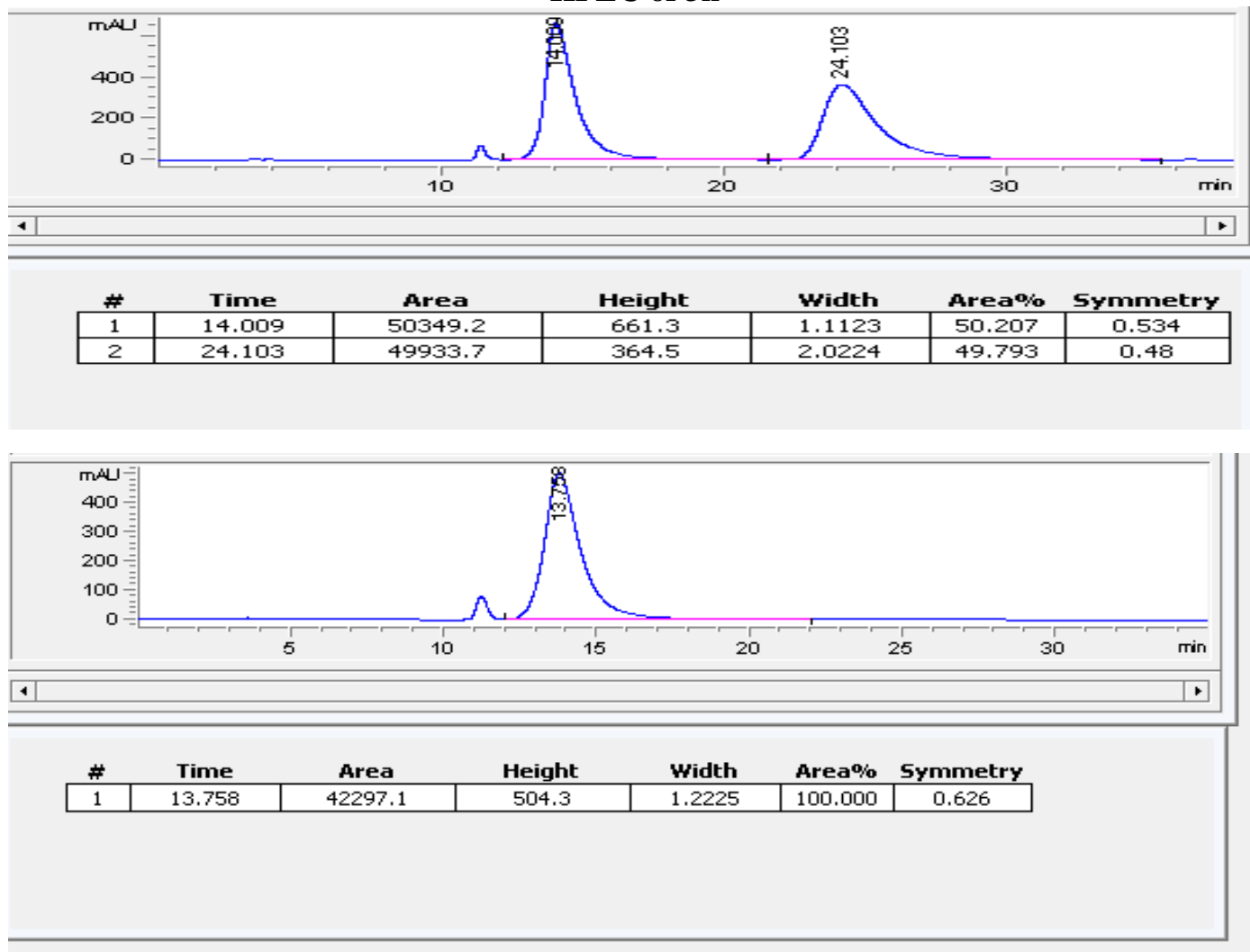


${ }^{1} \mathrm{H}$ and ${ }^{13} \mathrm{C}$ NMR of $3 i$

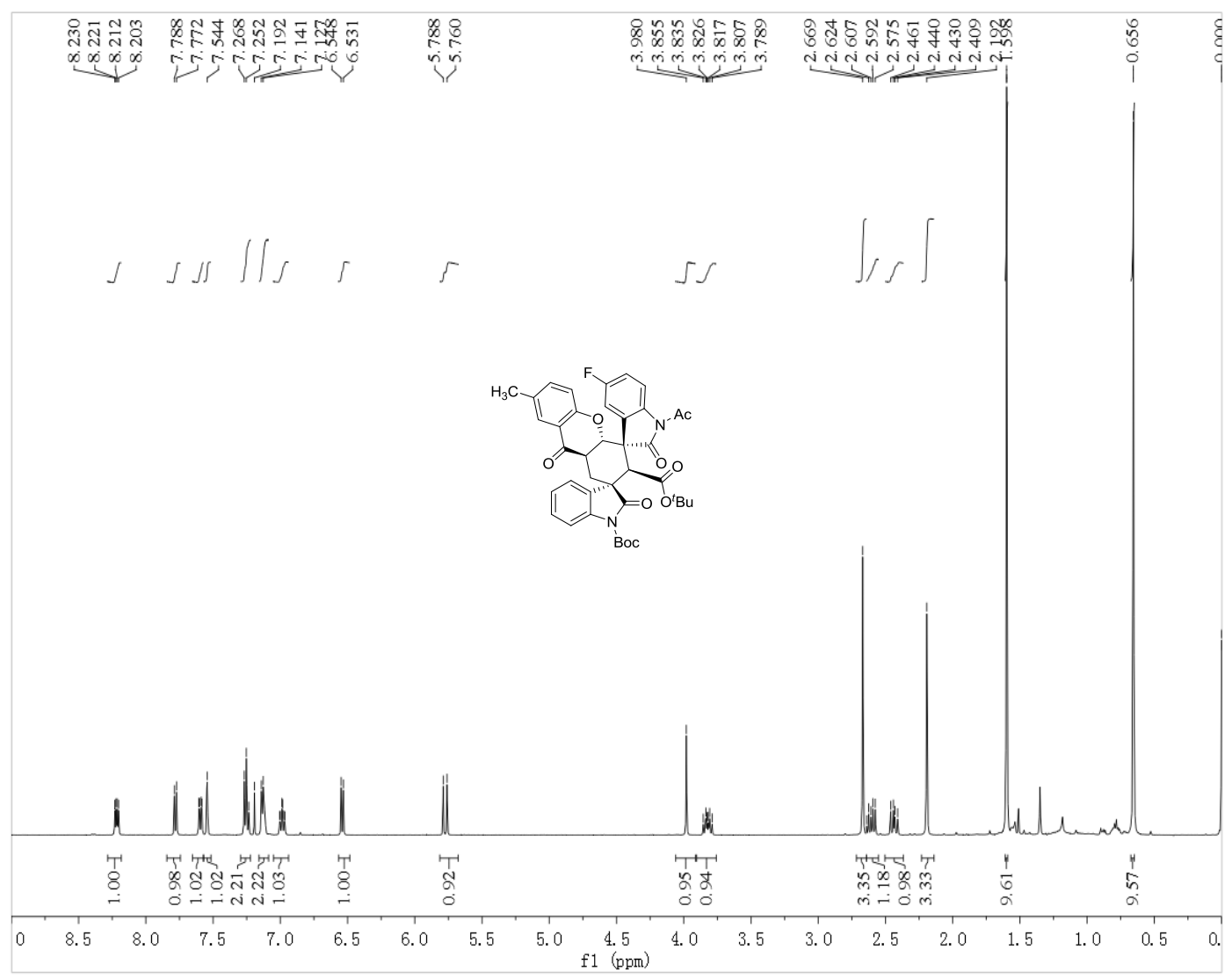

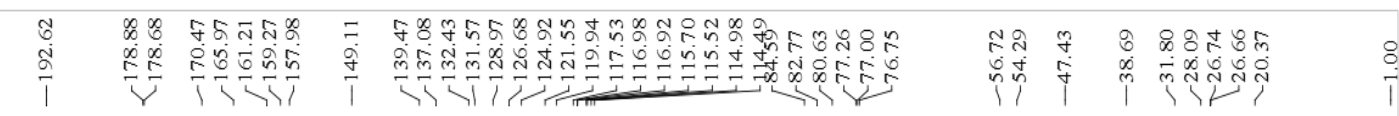
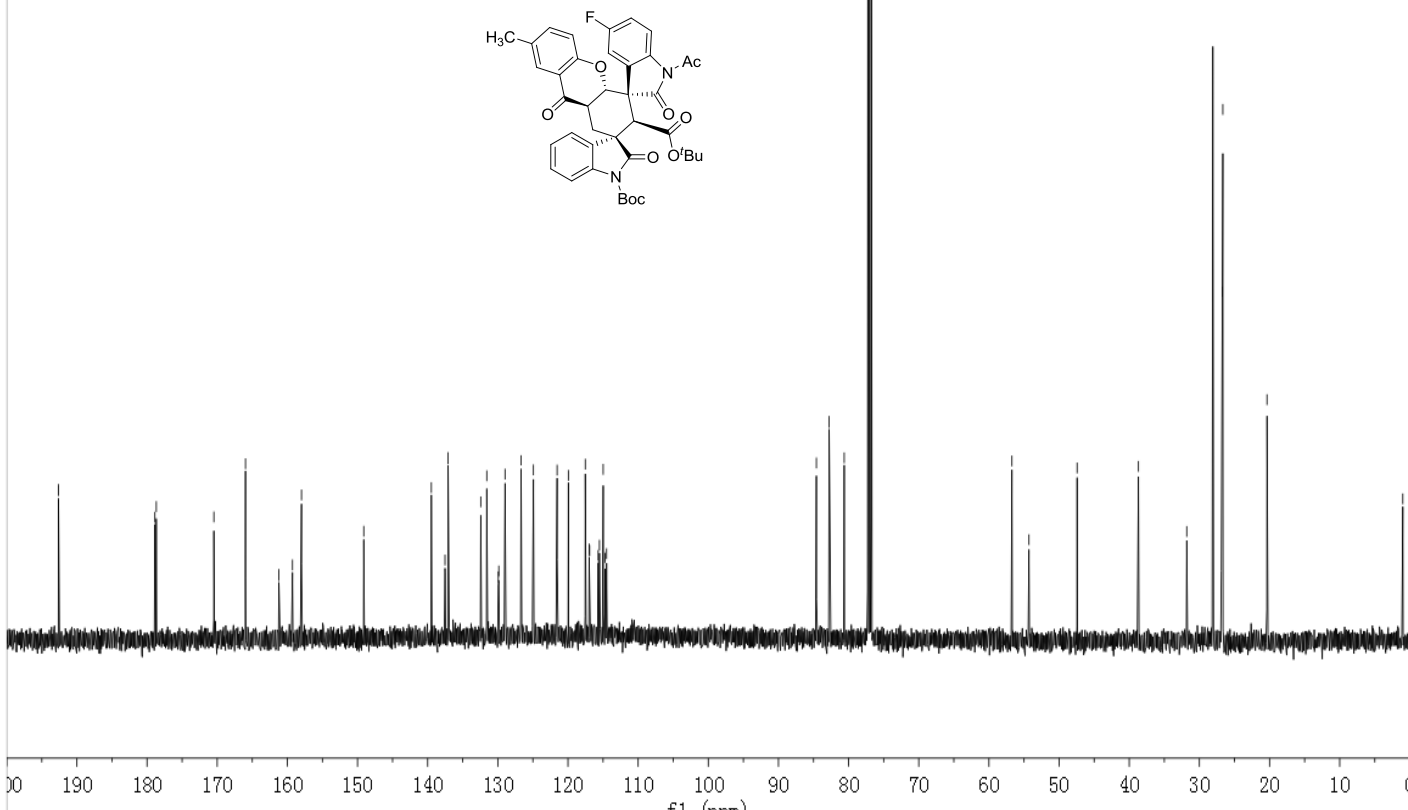
${ }^{19} \mathrm{~F}$ NMR of $3 \mathrm{i}$

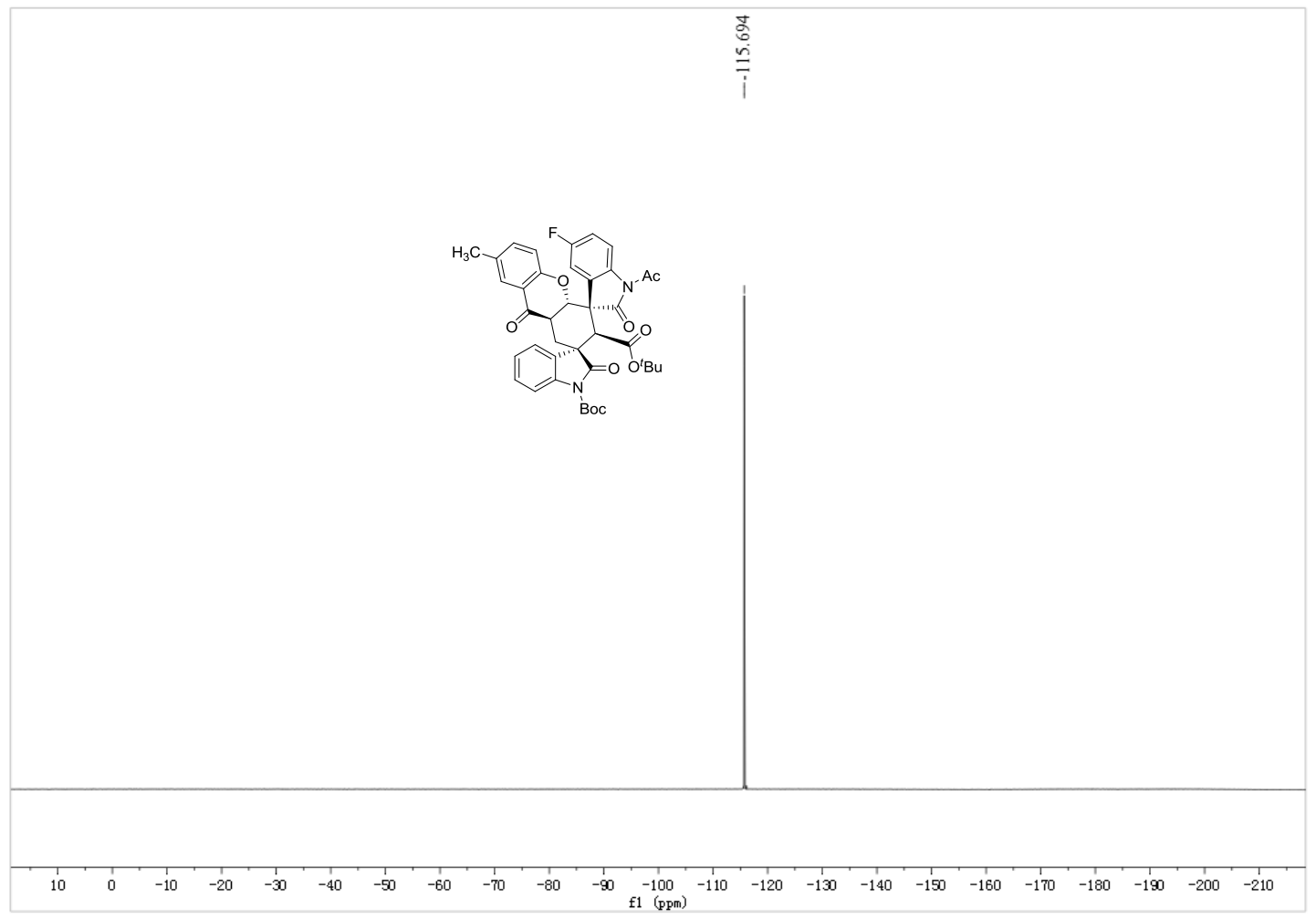

HPLC of 3i
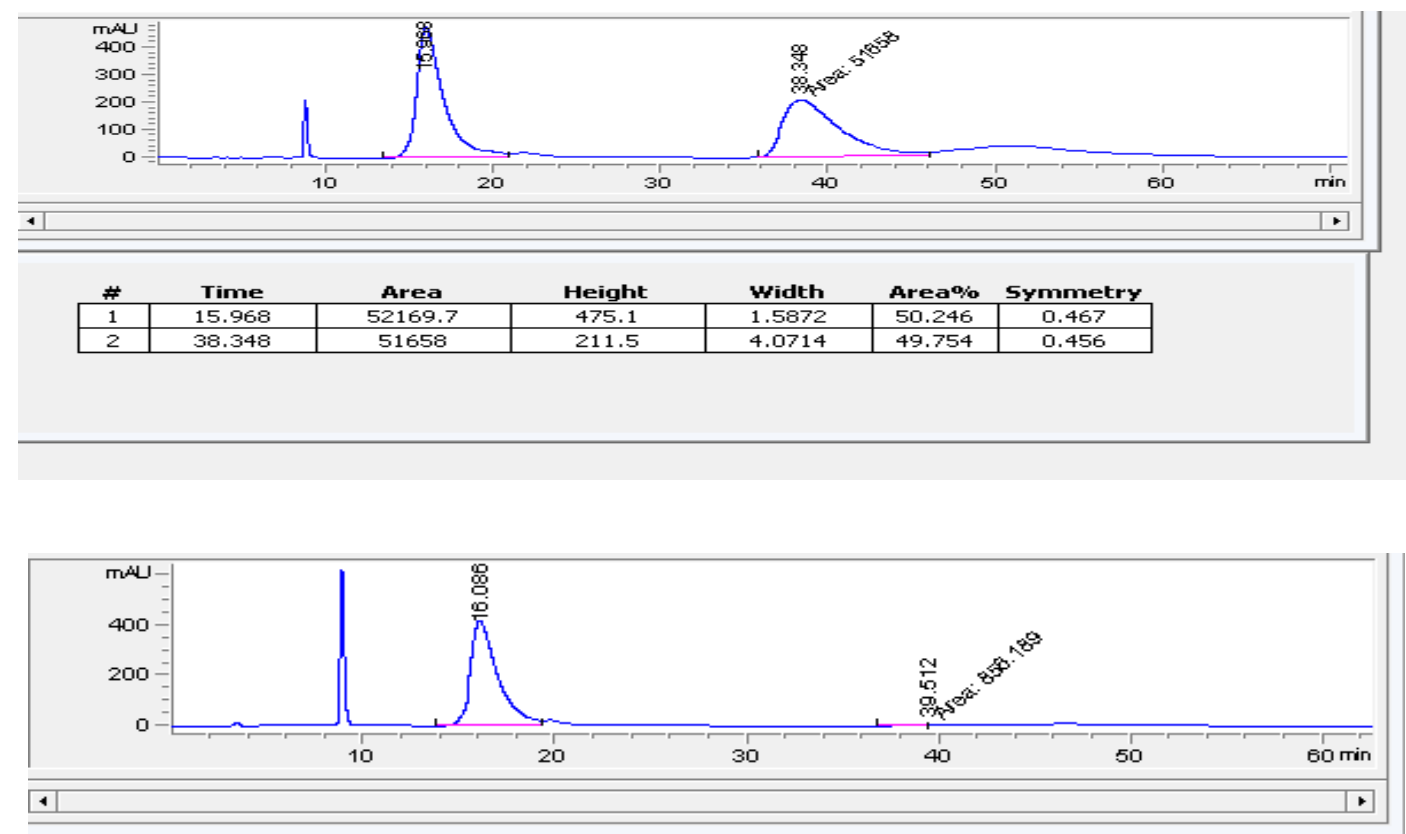

\begin{tabular}{|c|c|c|c|c|c|c|} 
\# & \multicolumn{1}{c}{ Time } & \multicolumn{1}{c}{ Area } & Height & Width & \multicolumn{2}{c|}{ Area\% } \\
\hline 1 & 16.086 & 42563.1 & 417.6 & 1.4732 & 98.028 & 0.514 \\
\hline 2 & 39.512 & 856.2 & 7.7 & 1.8622 & 1.972 & 0 \\
\hline
\end{tabular}


${ }^{1} \mathrm{H}$ and ${ }^{13} \mathrm{C}$ NMR of $3 \mathrm{j}$
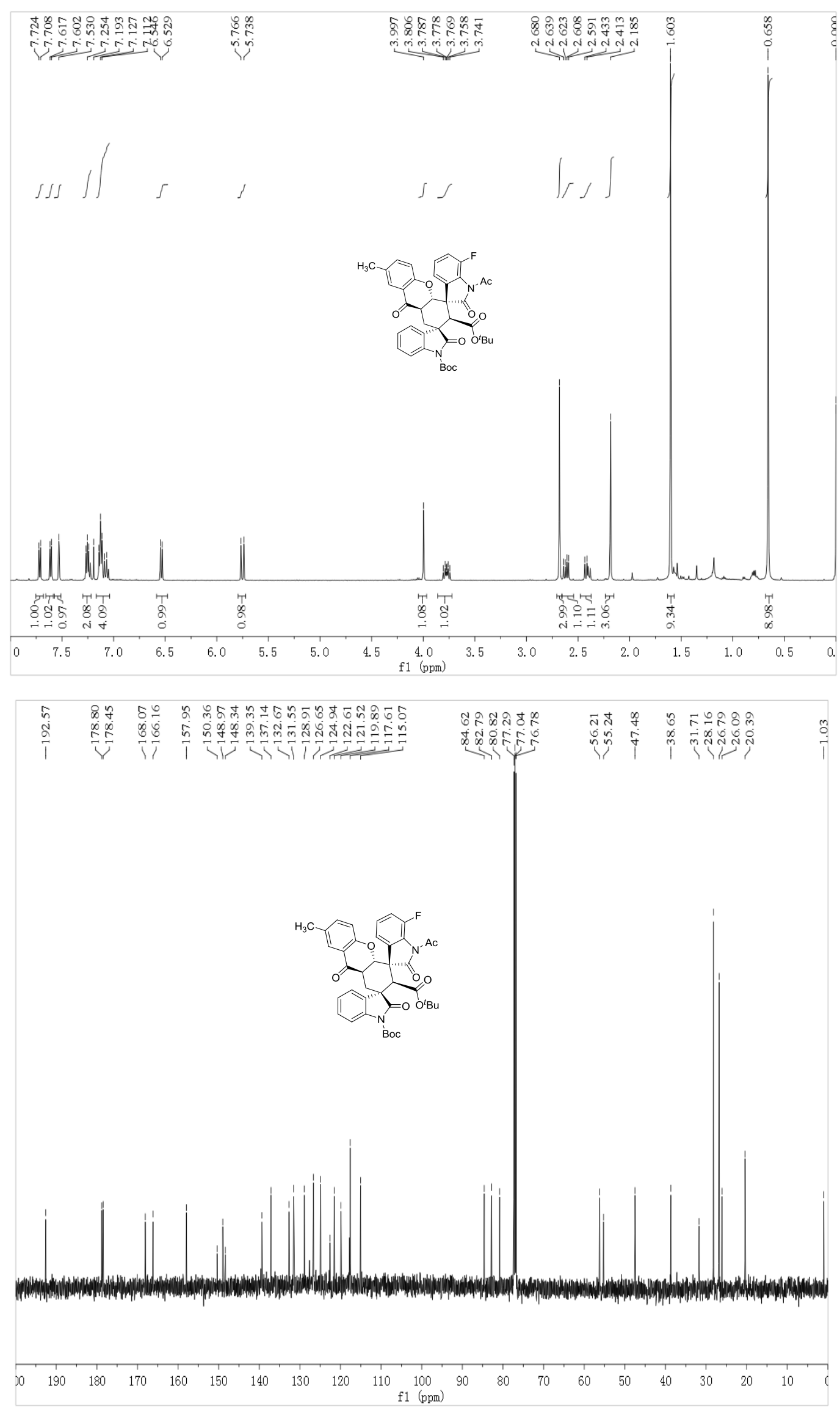


\section{${ }^{19}$ F NMR of $3 j$}

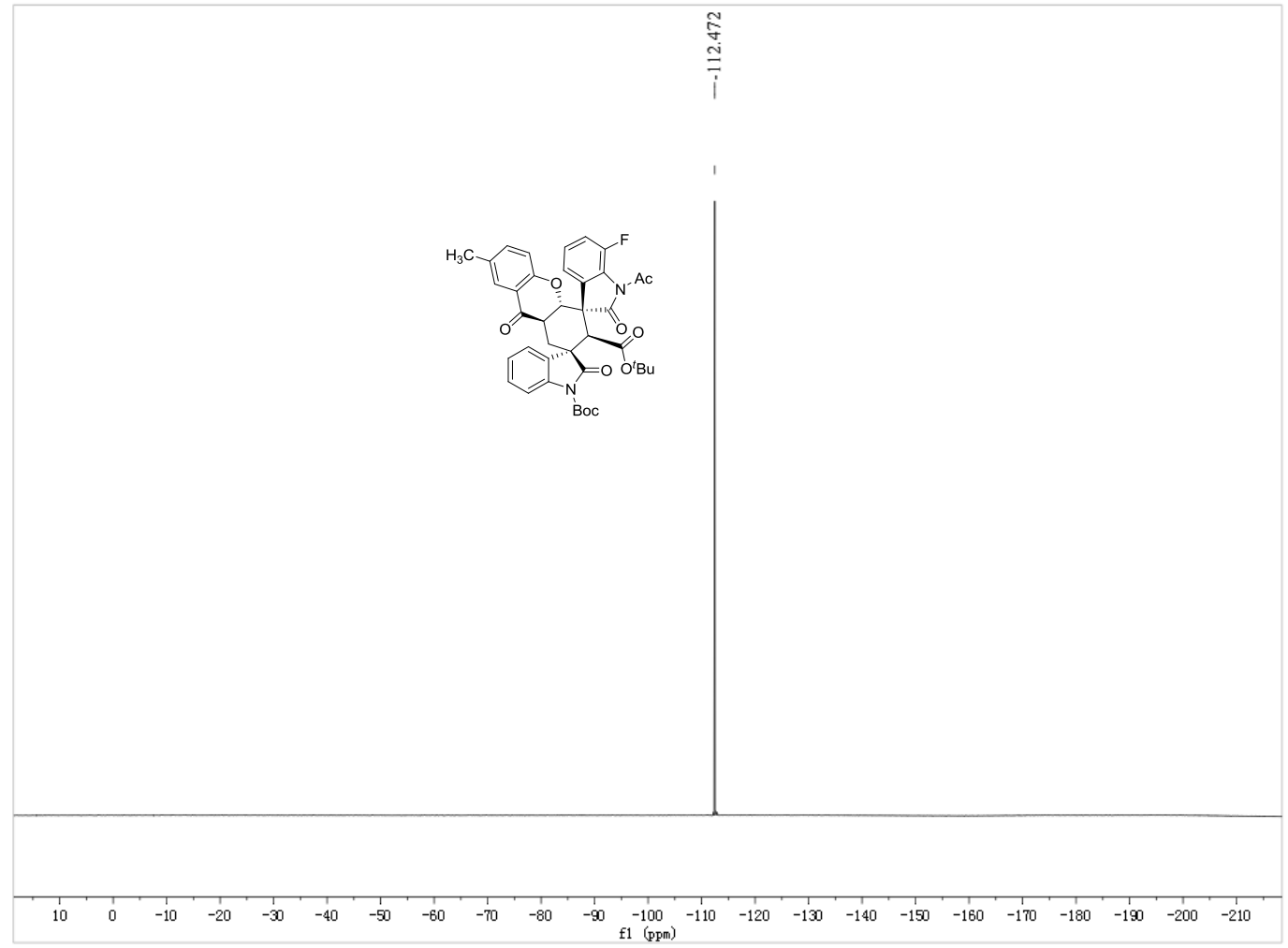

\section{HPLC of $\mathbf{3 j}$}
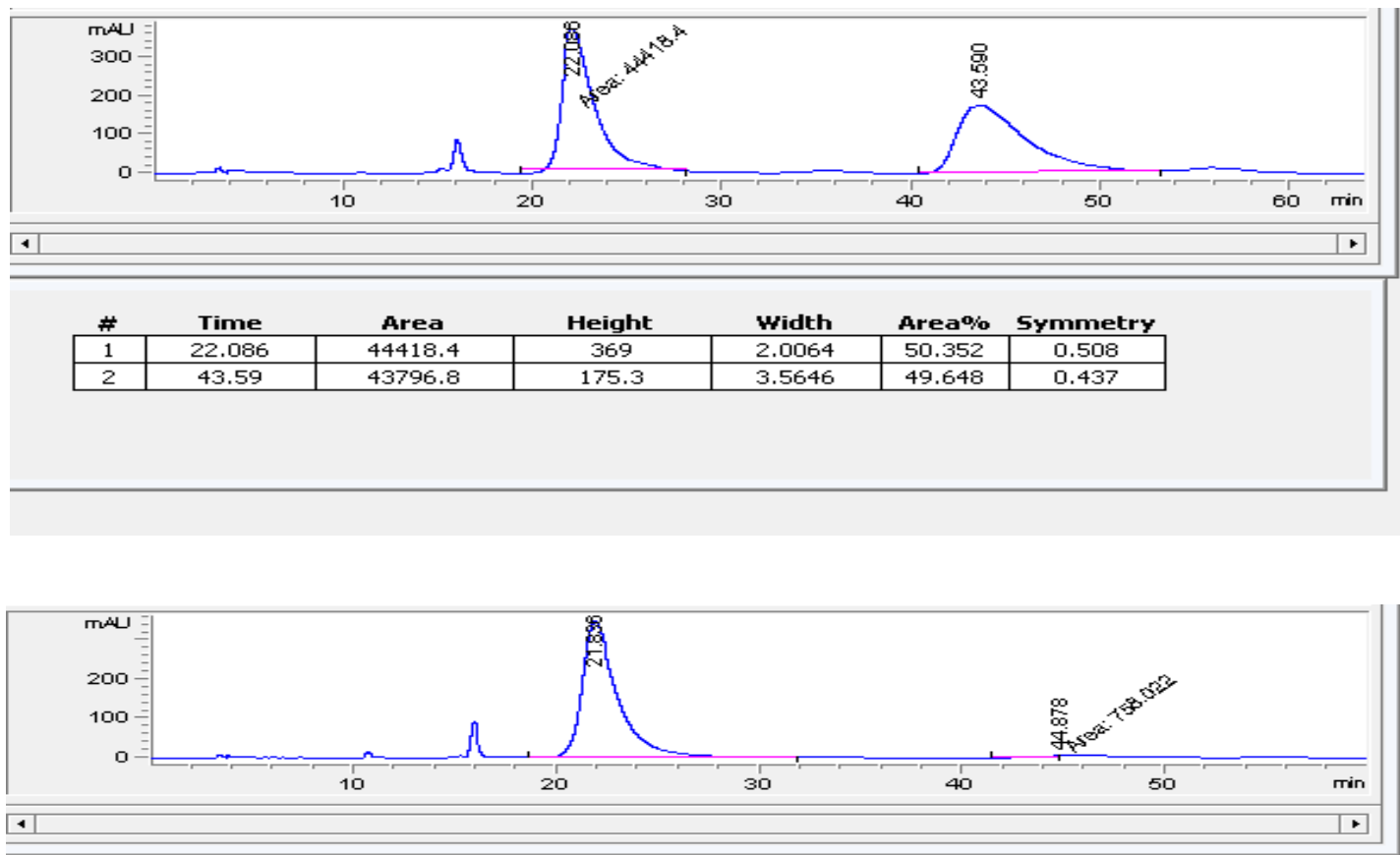

\begin{tabular}{|c|c|c|c|c|c|c|}
\hline$\#$ & Time & Area & Height & Width & Area\% & Symmetry \\
\hline 1 & 21.836 & 43290.6 & 347.2 & 1.8186 & 98.279 & 0.519 \\
\hline 2 & 44.878 & 758 & 7.3 & 1.7386 & 1.721 & 0 \\
\hline
\end{tabular}


${ }^{1} \mathrm{H}$ and ${ }^{13} \mathrm{C}$ NMR of $3 \mathrm{k}$

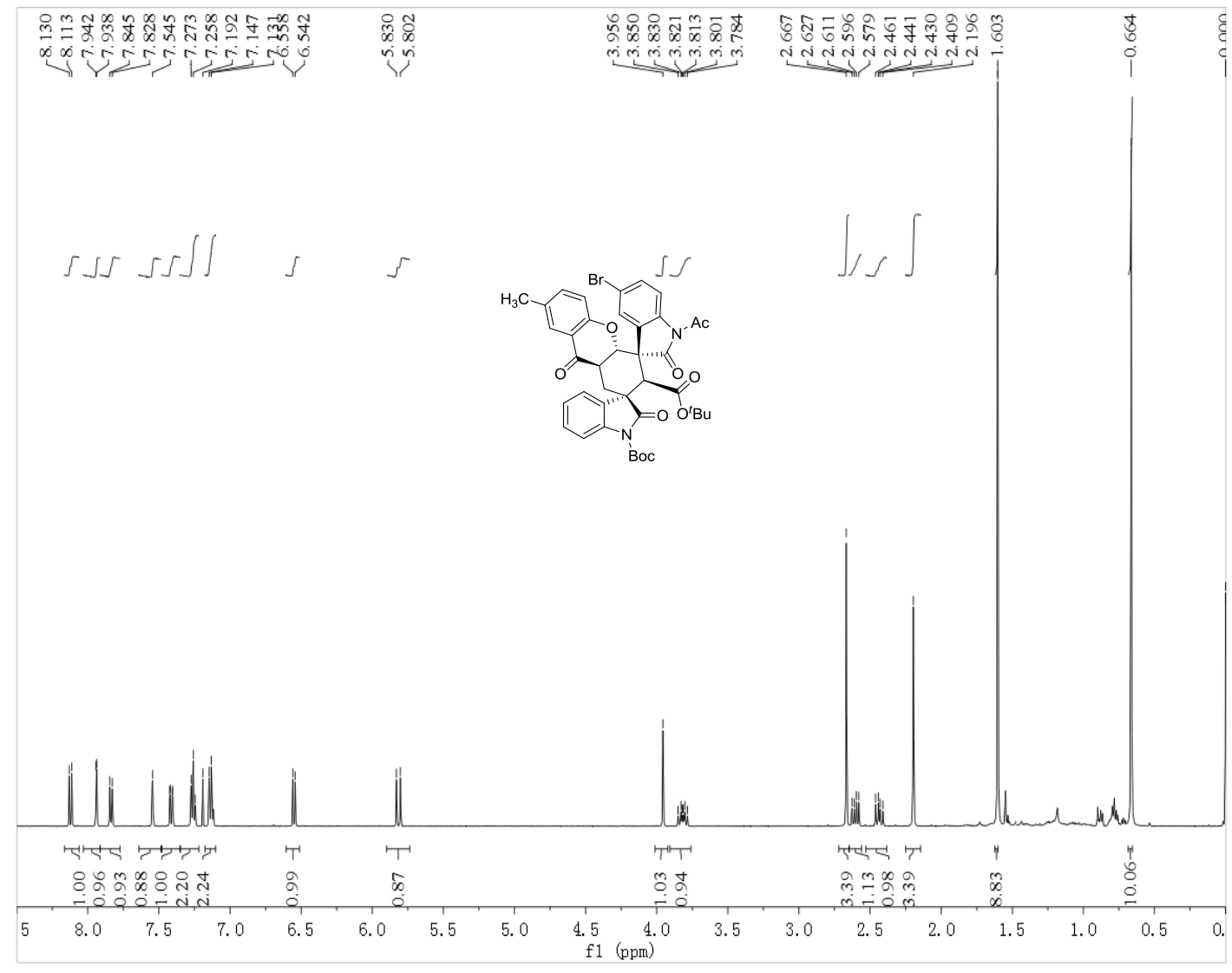

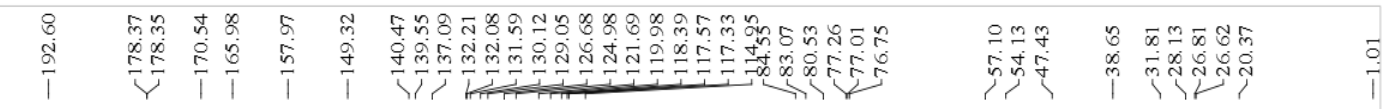

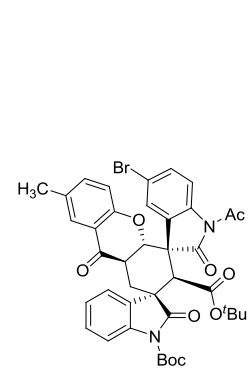

Boc

$\begin{array}{llllllllllllllllllll}10 & 190 & 180 & 170 & 160 & 150 & 140 & 130 & 120 & 110 & \underset{\mathrm{f} 1}{100}(\mathrm{ppm}) & 90 & 80 & 70 & 60 & 50 & 40 & 30 & 20 & 10\end{array}$ 
HPLC of 3k
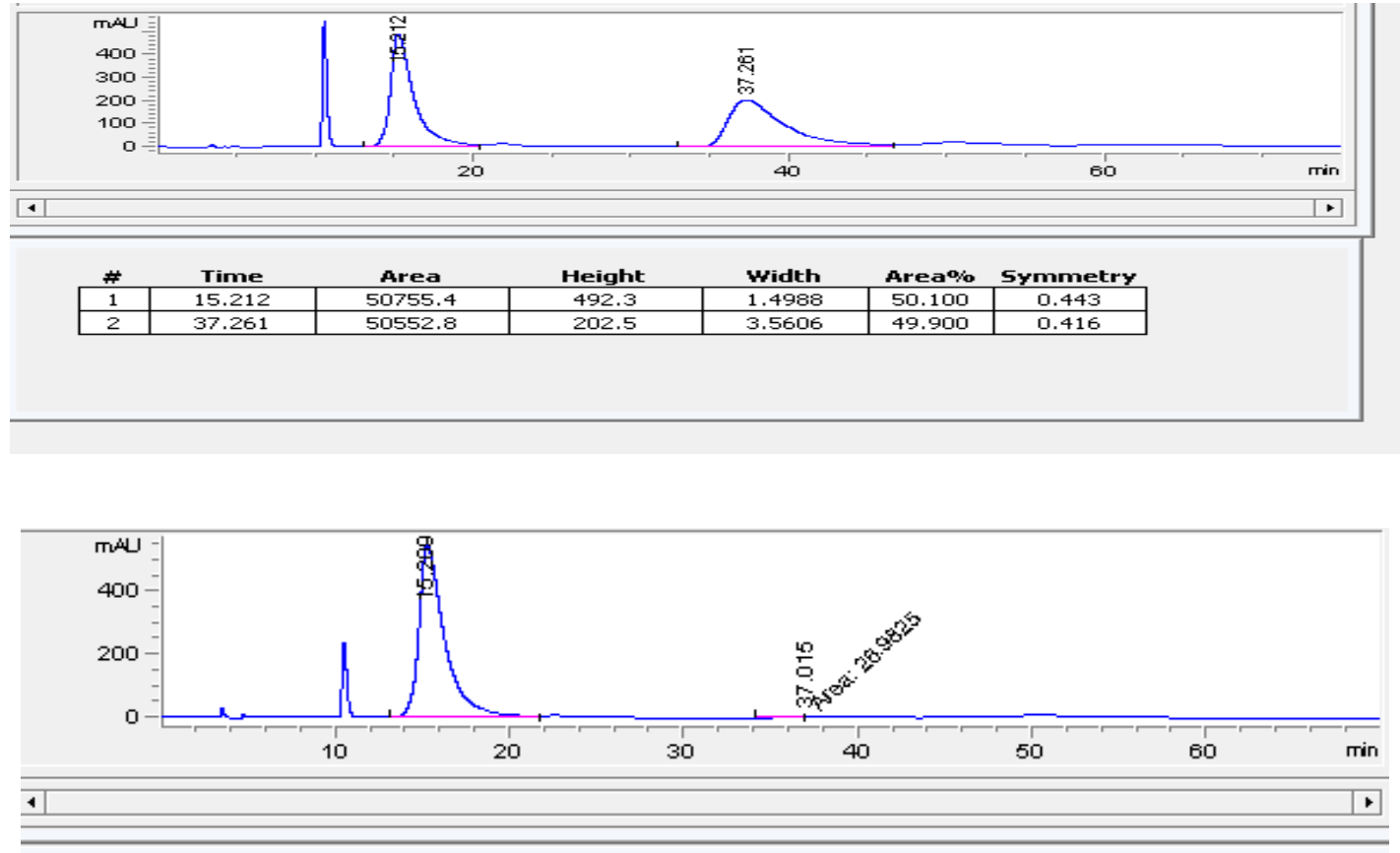

\begin{tabular}{|c|c|c|c|c|c|c|} 
\# & \multicolumn{1}{c}{ Time } & \multicolumn{1}{c}{ Area } & Height & \multicolumn{2}{c|}{ Width } & \multicolumn{2}{c|}{ Area\% $\%$} & Symmetry \\
\hline 1 & 15.209 & 56488.9 & 553.3 & 1.4871 & 99.952 & 0.446 \\
\hline 2 & 37.015 & 27 & 1.3 & 0.3565 & 0.048 & 0 \\
\hline
\end{tabular}


${ }^{1} \mathrm{H}$ and ${ }^{13} \mathrm{C}$ NMR of 31

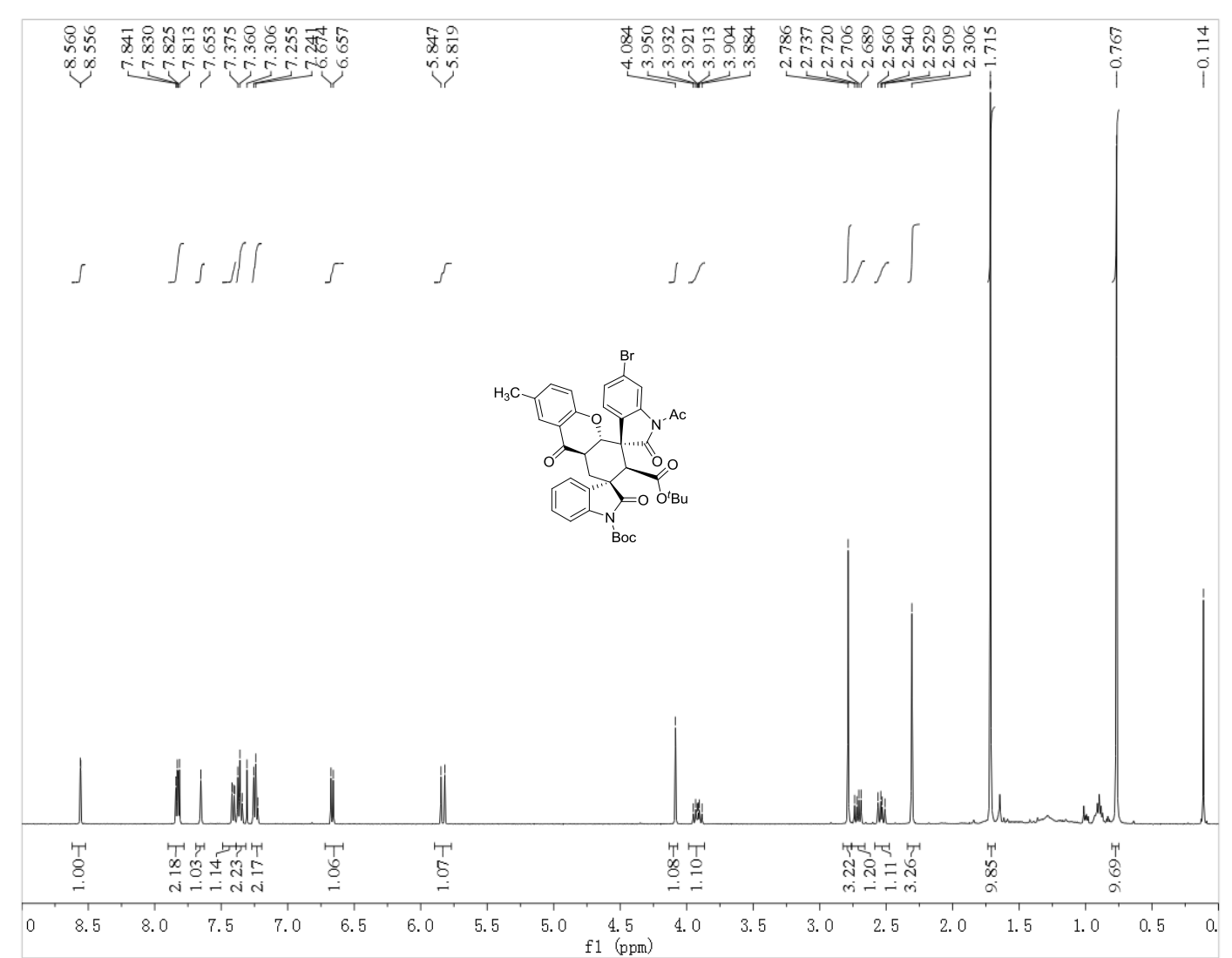

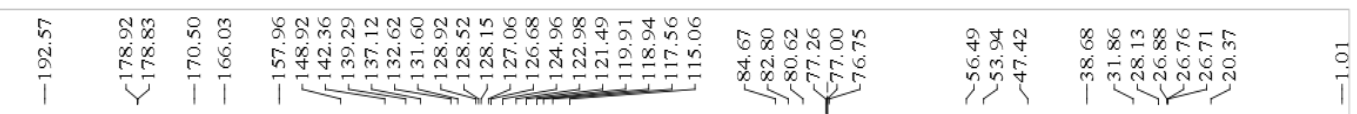
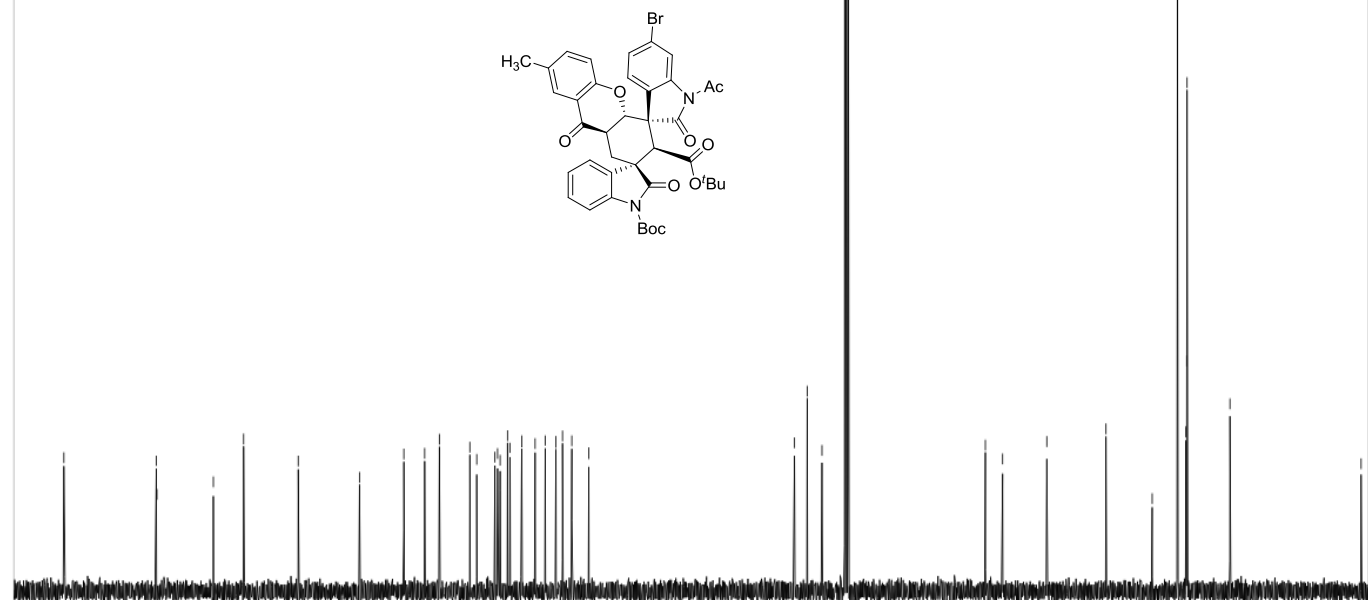

$\begin{array}{llllllllllllllllllllll}30 & 190 & 180 & 170 & 160 & 150 & 140 & 130 & 120 & 110 & 100 & 90 & 80 & 70 & 60 & 50 & 40 & 30 & 20 & 10 & \end{array}$ 
HPLC of 31
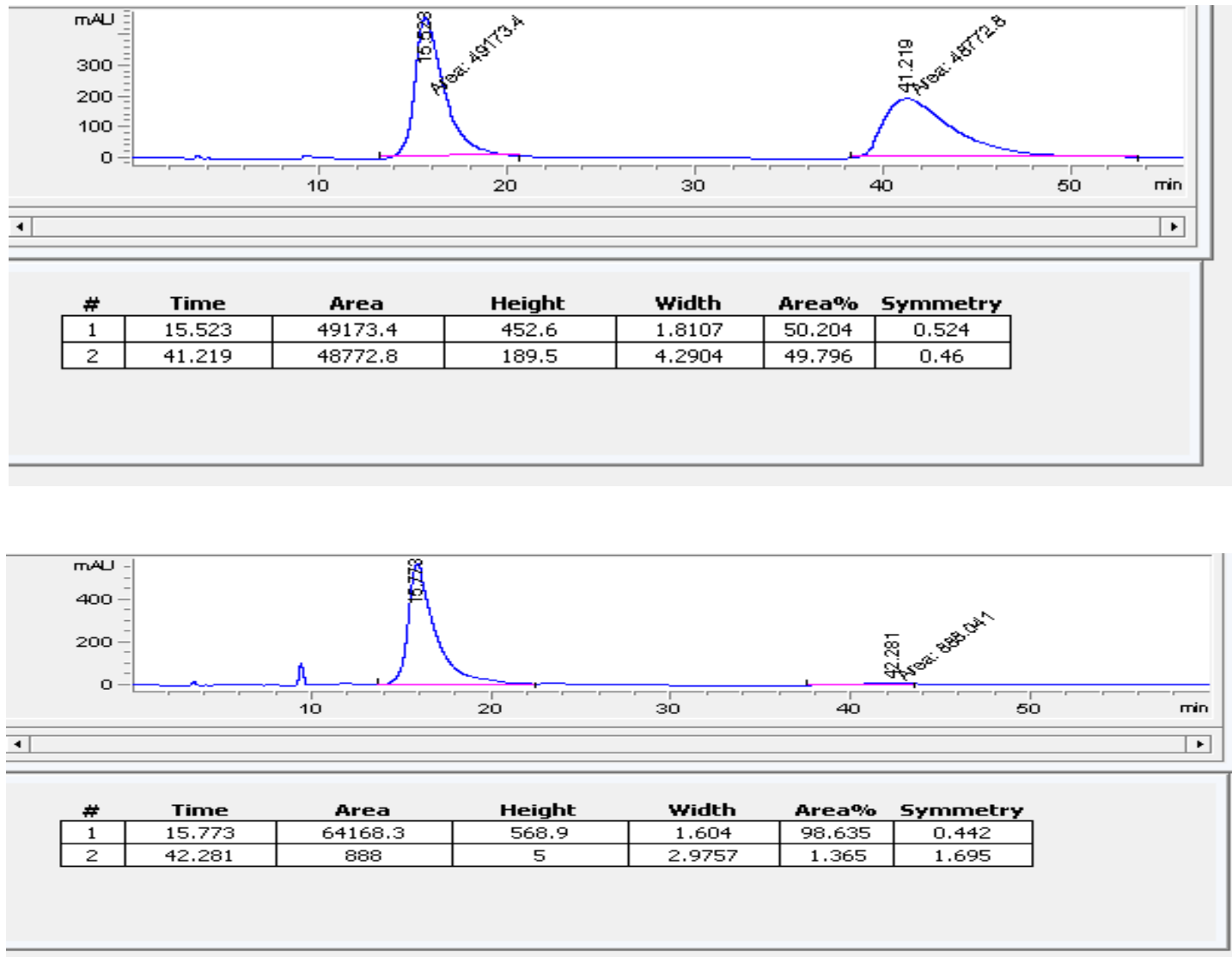


\section{${ }^{1} \mathrm{H}$ and ${ }^{13} \mathrm{C}$ NMR of $3 \mathrm{~m}$}
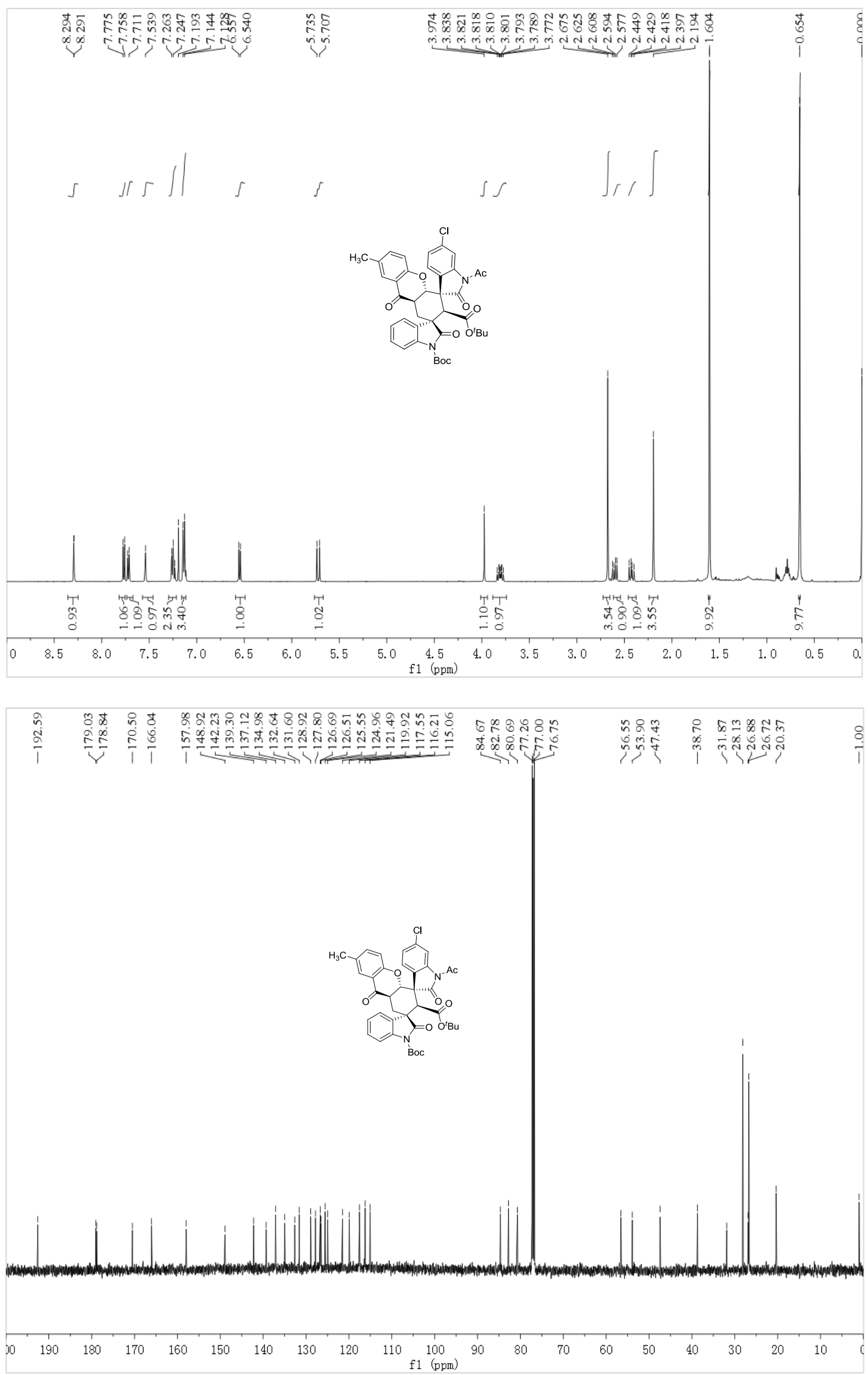
HPLC of 3m
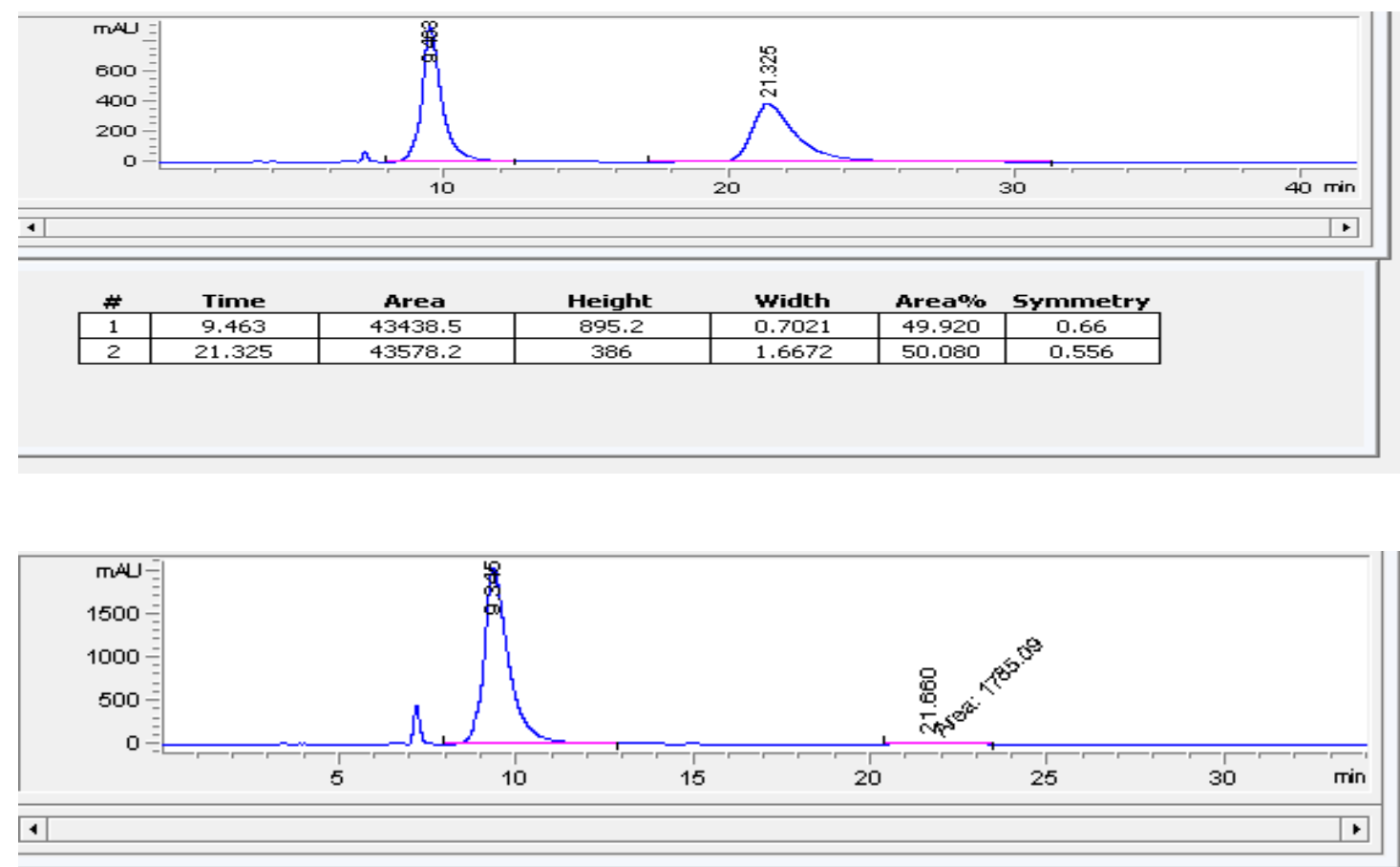

\begin{tabular}{|c|c|c|c|c|c|c|}
\hline$\#$ & Time & Area & Height & Width & Area\% & Symmetry \\
\hline 1 & 9.345 & 96990.4 & 2018.1 & 0.6987 & 98.193 & 0.586 \\
\hline 2 & 21.66 & 1785.1 & 18.5 & 1.6054 & 1.807 & 2.035 \\
\hline
\end{tabular}




\section{${ }^{1} \mathrm{H}$ and ${ }^{13} \mathrm{C}$ NMR of $3 n$}

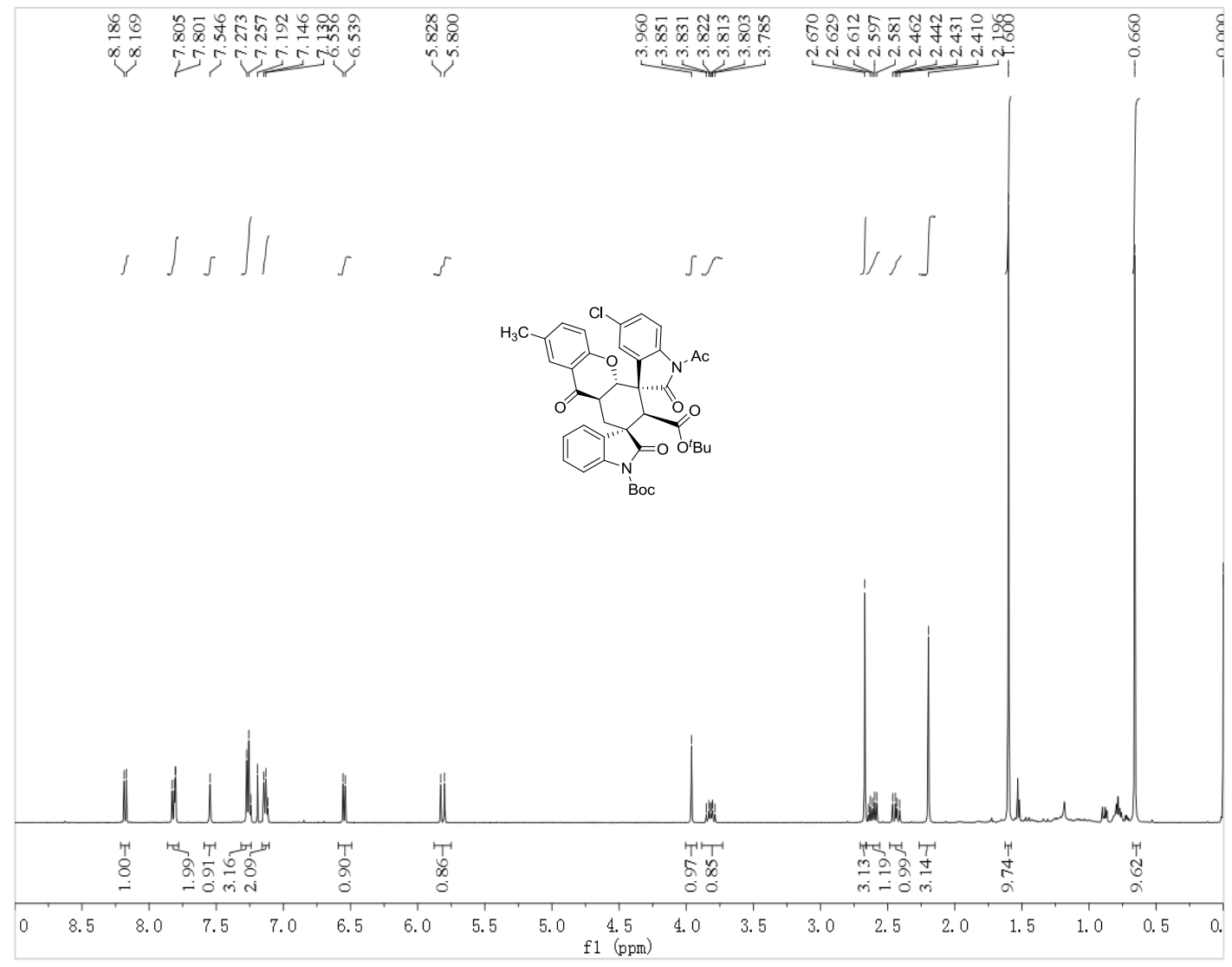

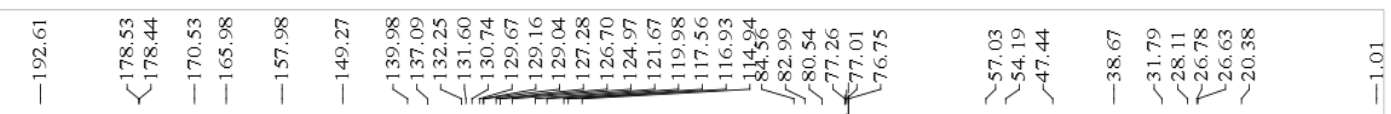

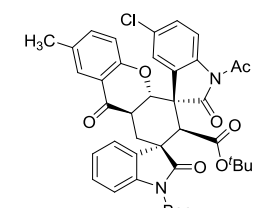

Boc
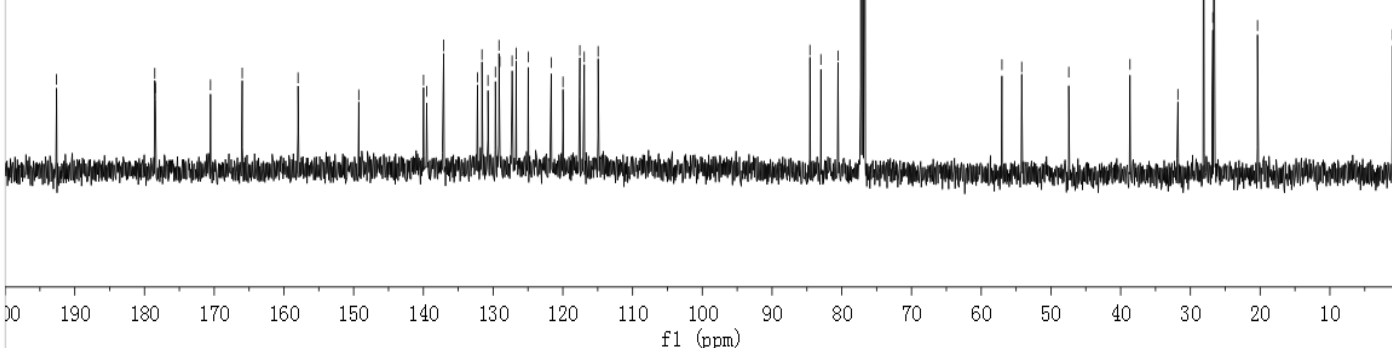
HPLC of 3n
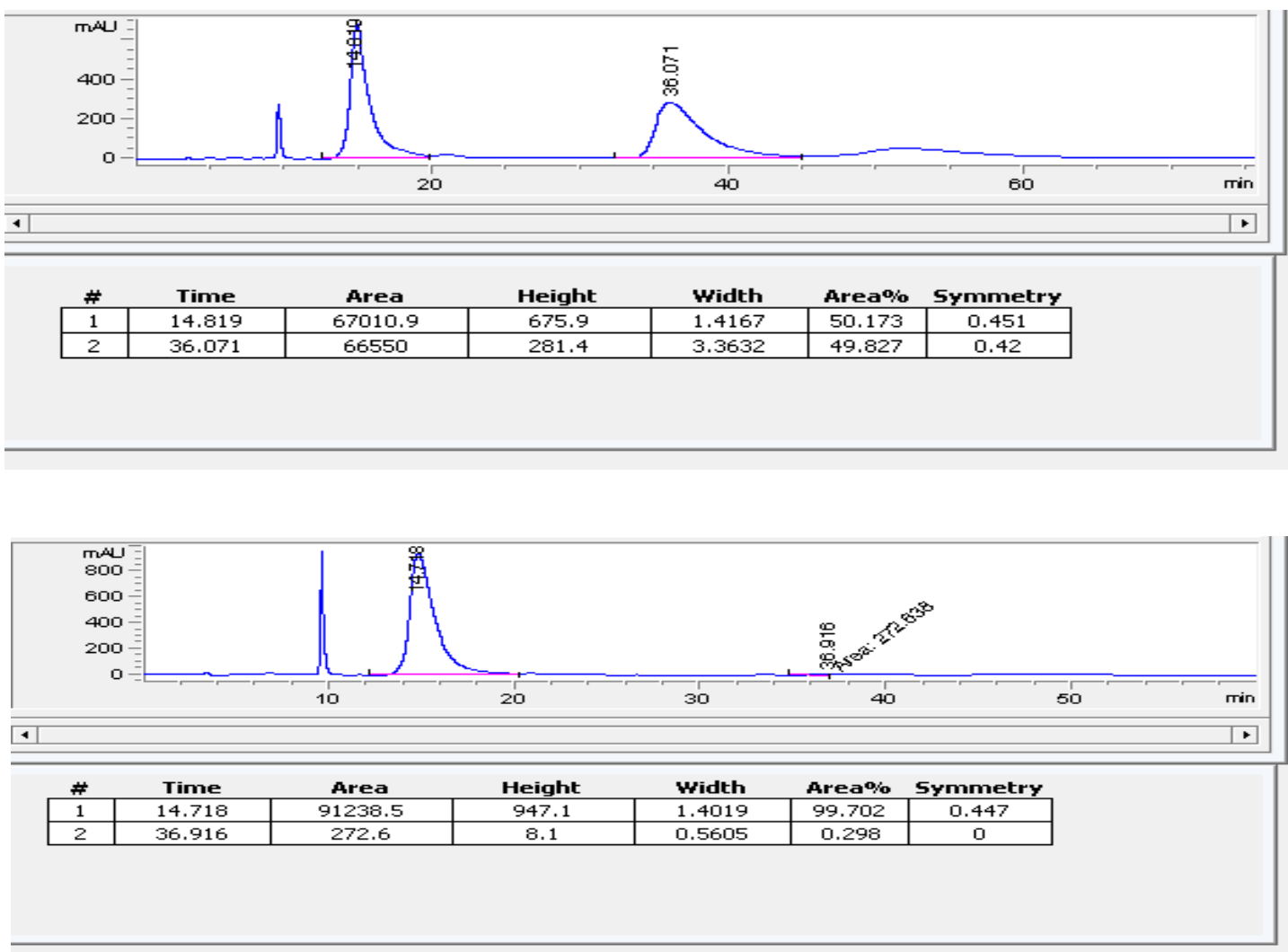
${ }^{1} \mathrm{H}$ and ${ }^{13} \mathrm{C}$ NMR of 30

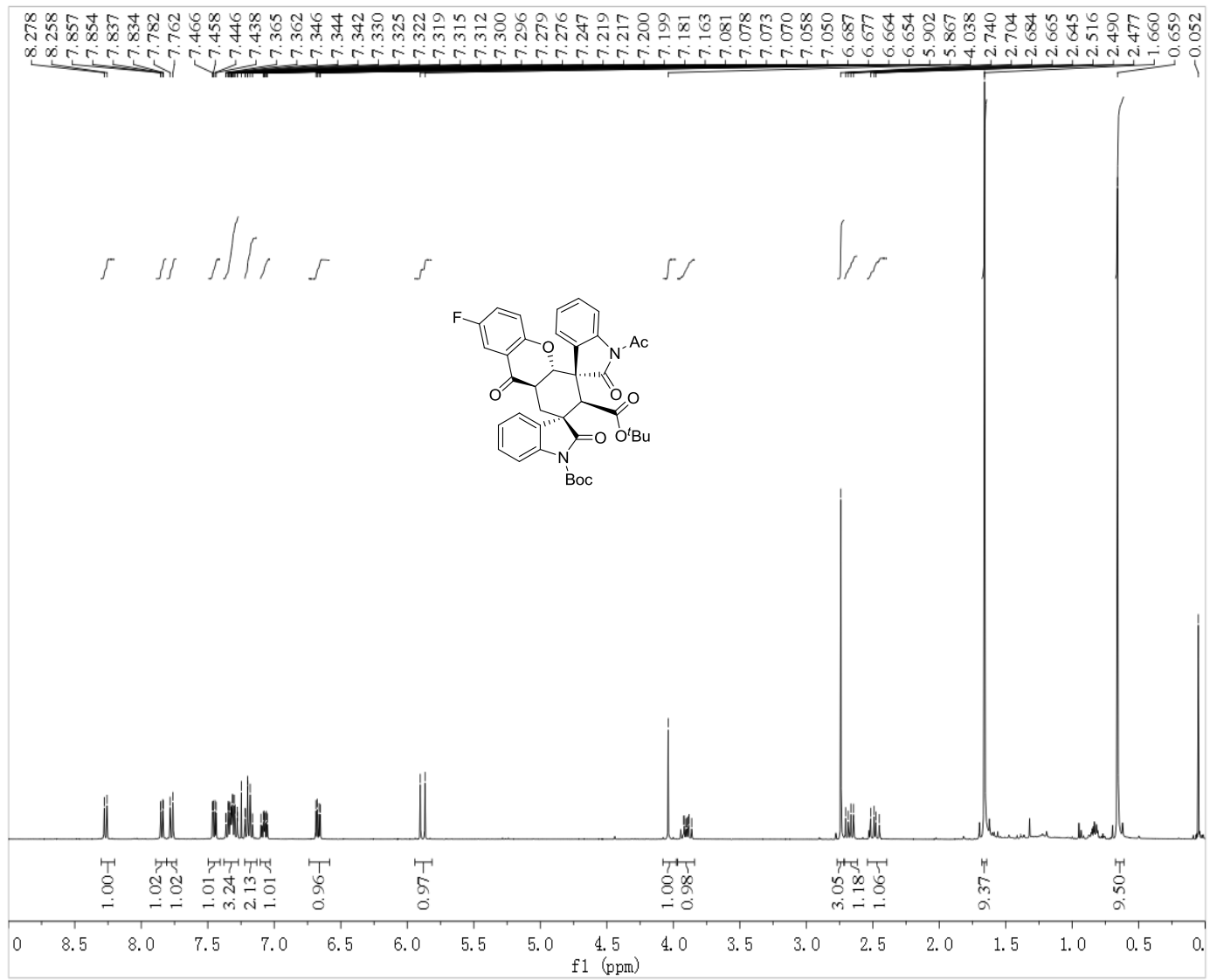

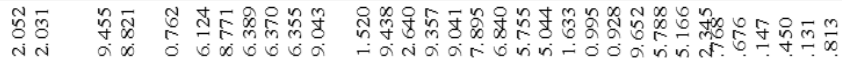

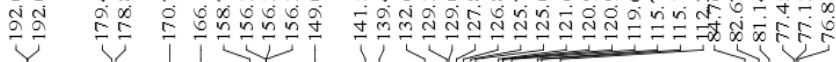

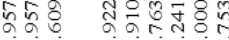

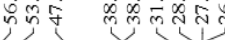

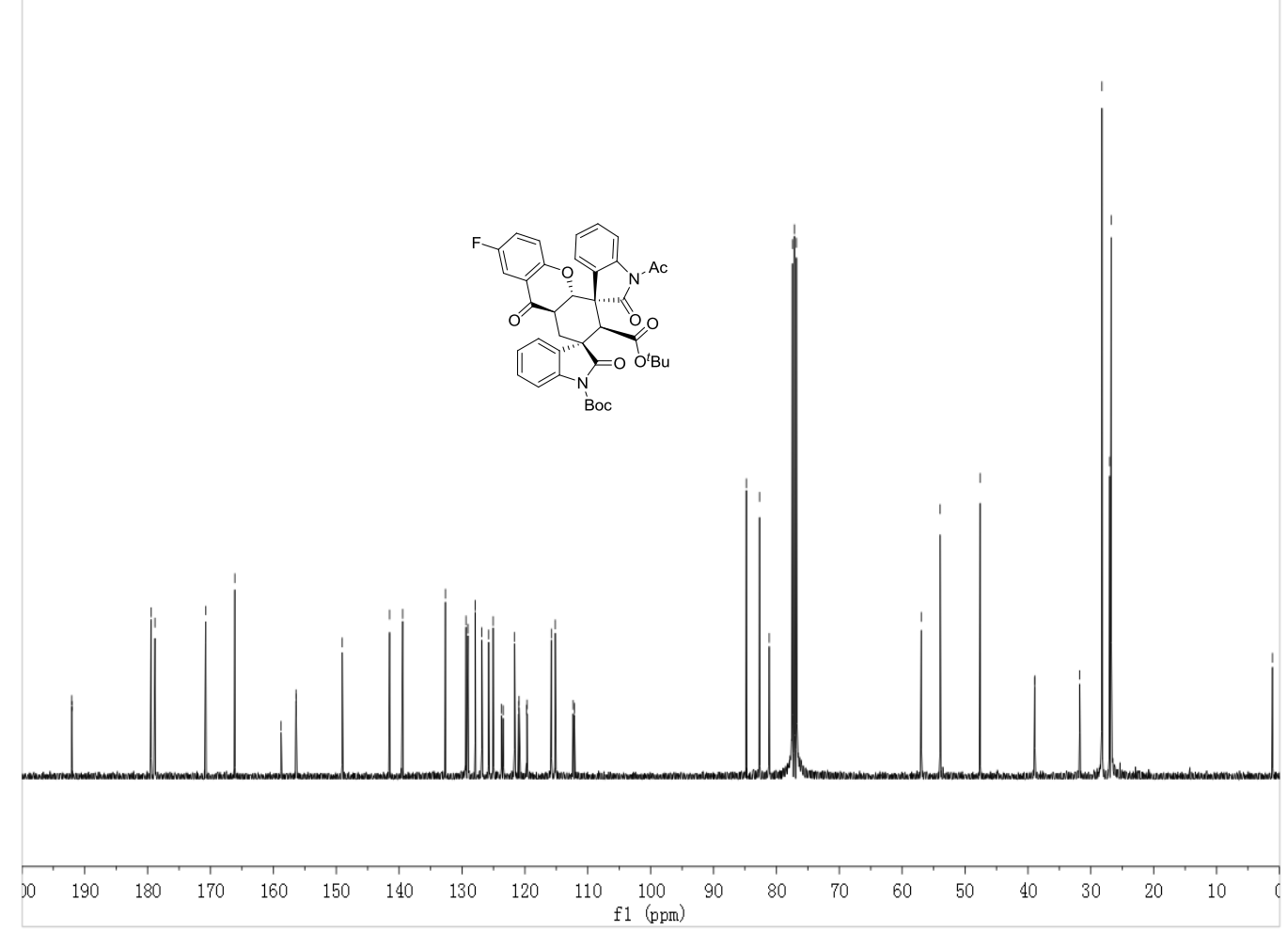




\section{${ }^{19}$ F NMR of 30}

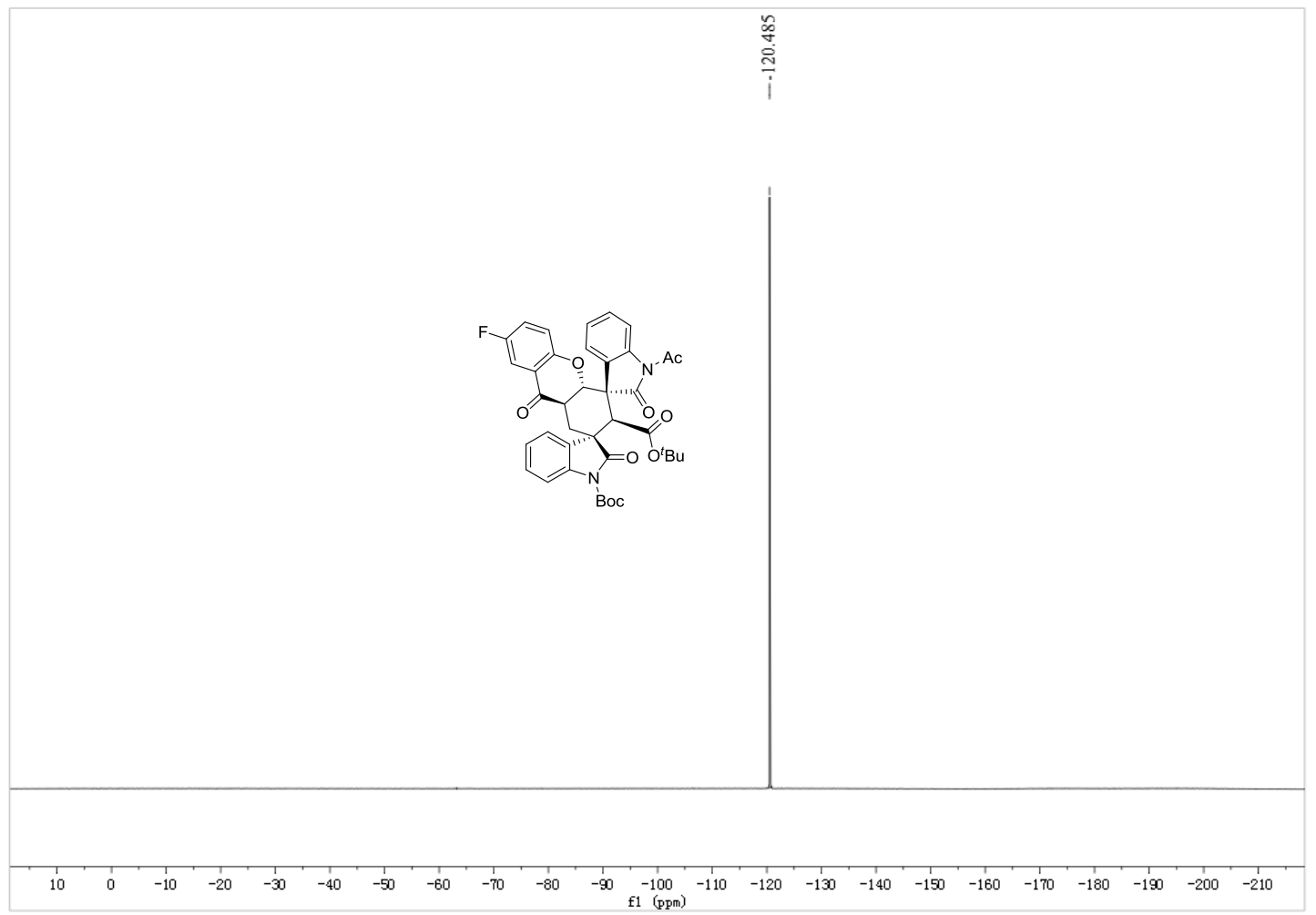

\section{HPLC of 30}

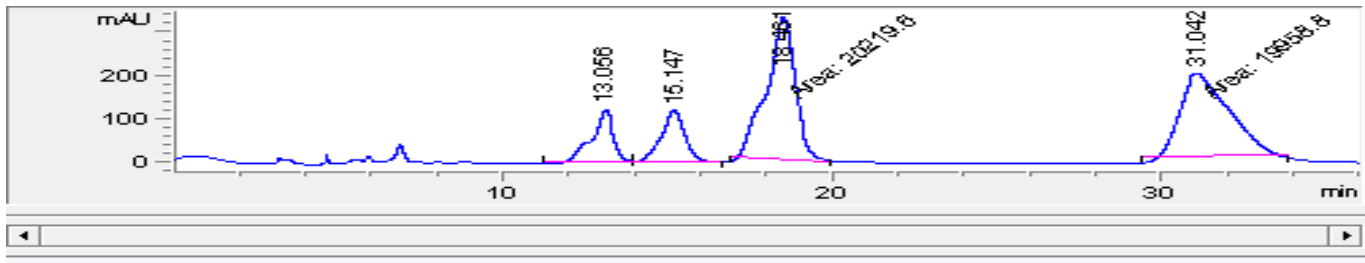

\begin{tabular}{|c|c|c|c|c|c|c|}
\hline$\#$ & Time & Area & Height & Width & Area\% & Symmetry \\
\hline 1 & 13.056 & 5984.9 & 123.4 & 0.6858 & 11.451 & 1.534 \\
\hline 2 & 15.147 & 6102.7 & 121.6 & 0.7214 & 11.676 & 1.147 \\
\hline 3 & 18.461 & 20219.6 & 330 & 1.0211 & 38.686 & 1.296 \\
\hline 4 & 31.042 & 19958.8 & 193.6 & 1.7185 & 38.187 & 0.526 \\
\hline
\end{tabular}

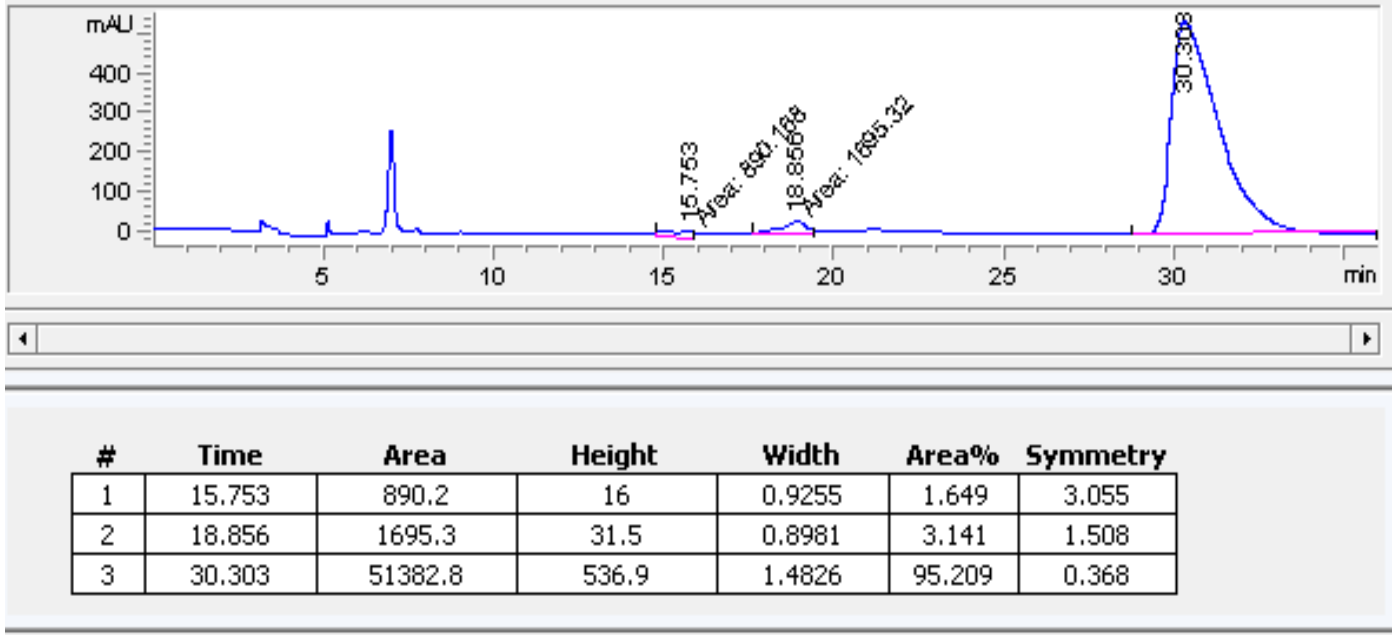


${ }^{1} \mathrm{H}$ and ${ }^{13} \mathrm{C}$ NMR of $3 p$

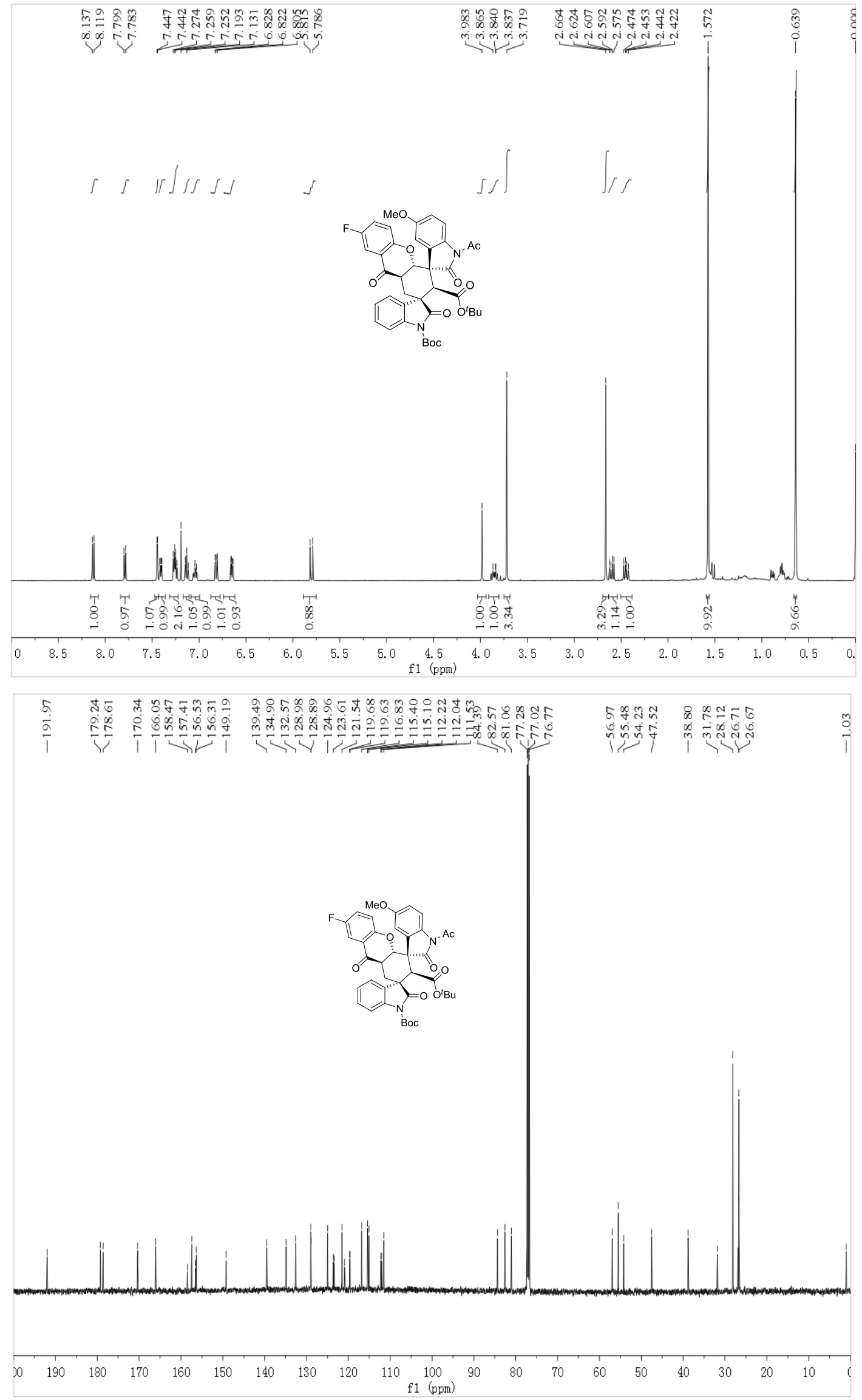




\section{${ }^{19}$ F NMR of 3p}

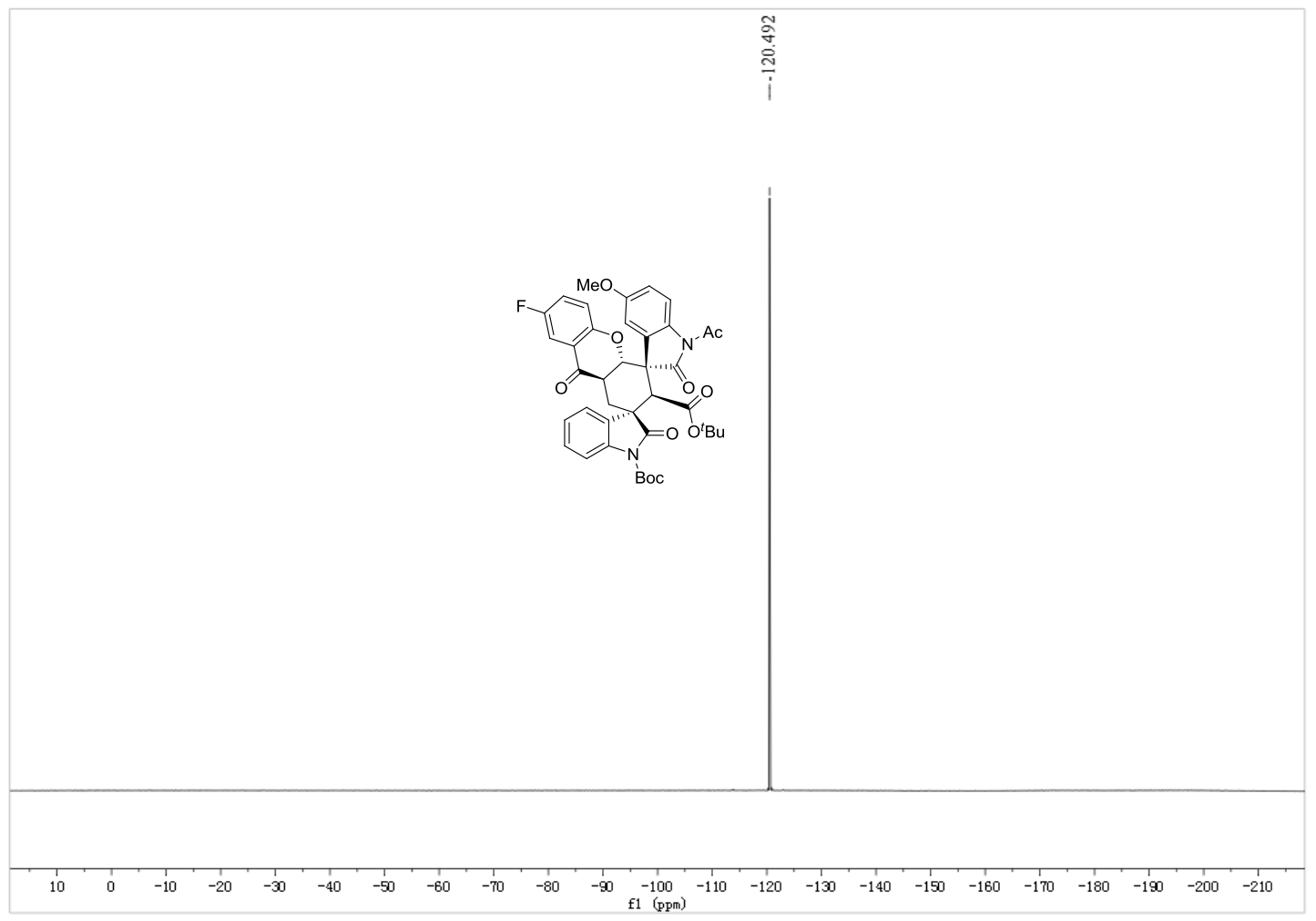

HPLC of 3p
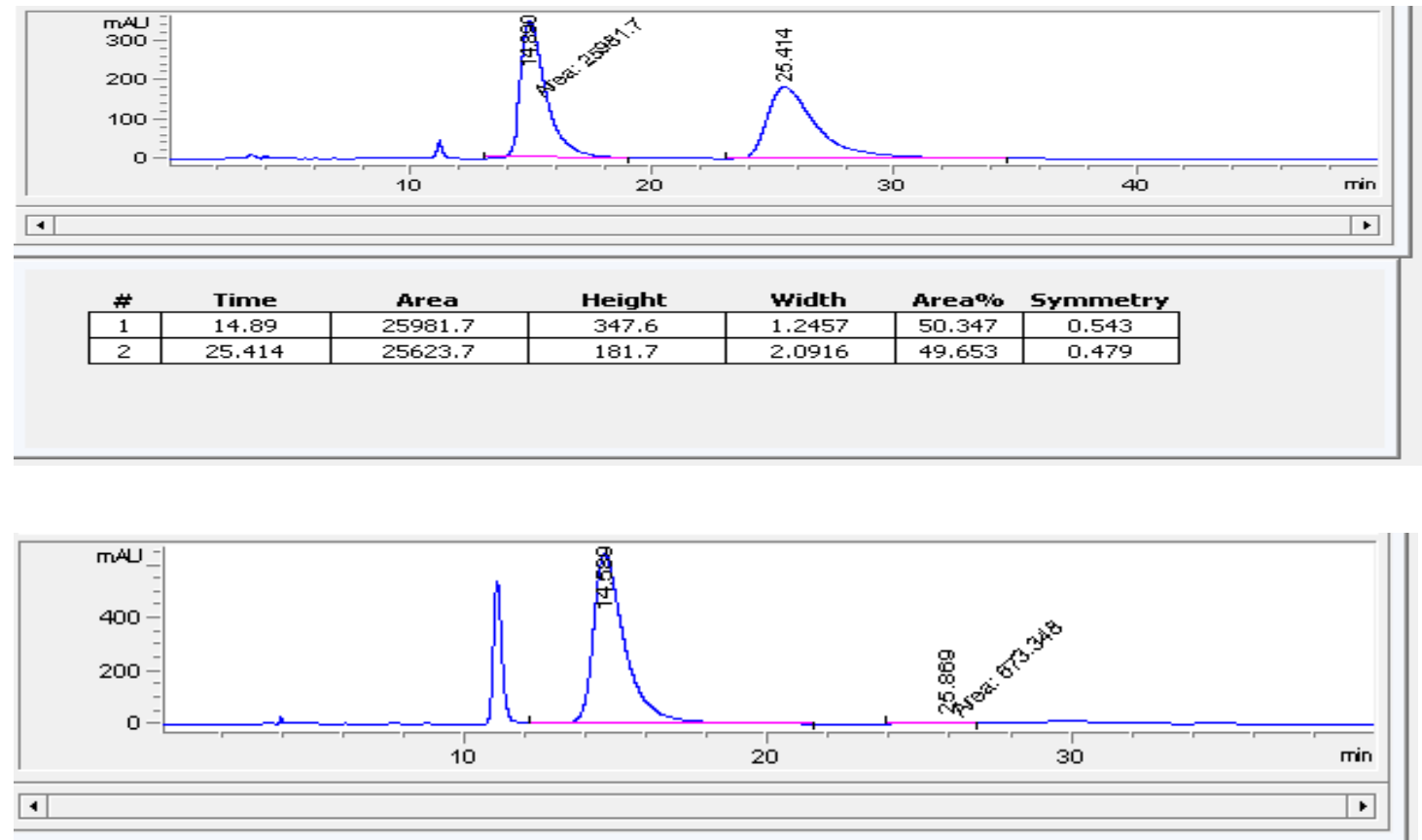

\begin{tabular}{|c|c|c|c|c|c|c|} 
\# & \multicolumn{1}{c}{ Time } & \multicolumn{1}{c}{ Area } & Height & \multicolumn{1}{c}{ Width } & \multicolumn{1}{c}{ Area\% } & Symmetry \\
\hline 1 & 14.589 & 46956.4 & 643.4 & 1.0834 & 98.586 & 0.517 \\
\hline 2 & 25.869 & 673.3 & 6.4 & 1.7548 & 1.414 & 0.445 \\
\hline
\end{tabular}




\section{${ }^{1} \mathrm{H}$ and ${ }^{13} \mathrm{C}$ NMR of $3 q$}
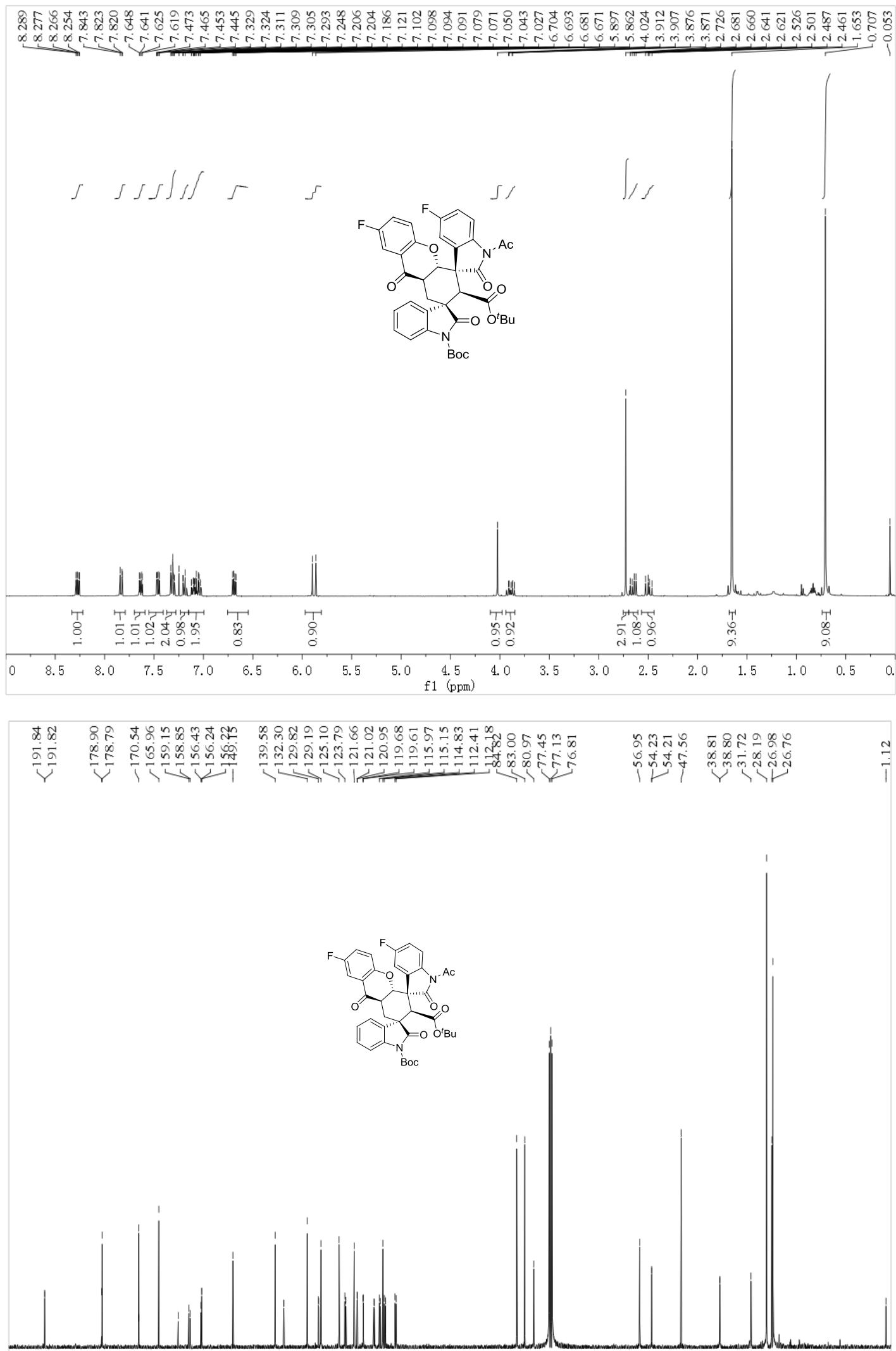

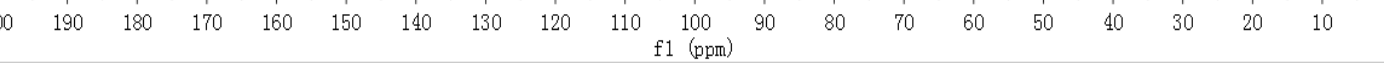




\section{${ }^{19}$ F NMR of 3q}

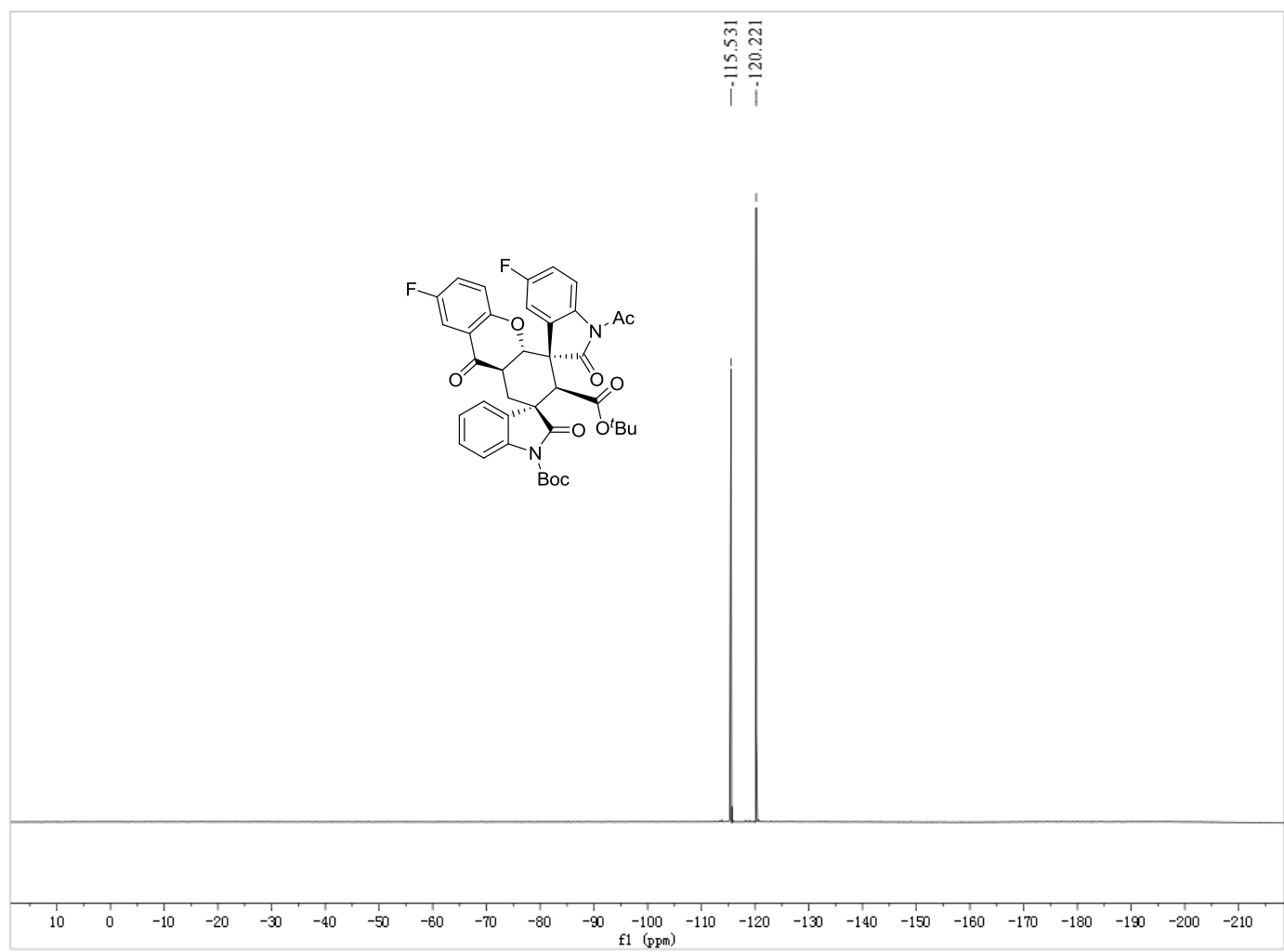

\section{HPLC of 3q}

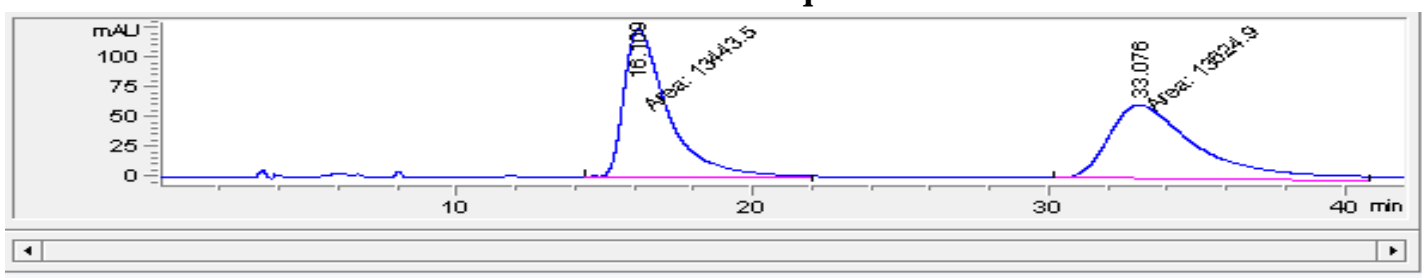

\begin{tabular}{|c|c|c|c|c|c|c|} 
\# & Time & \multicolumn{1}{c}{ Area } & Height & Width & \multicolumn{2}{c}{ Area\% } \\
\hline 1 & 16.109 & 13443.5 & 126 & 1.7784 & 49.665 & 0.465 \\
\hline 2 & 33.076 & 13624.9 & 62.8 & 3.6162 & 50.335 & 0.513 \\
\hline
\end{tabular}

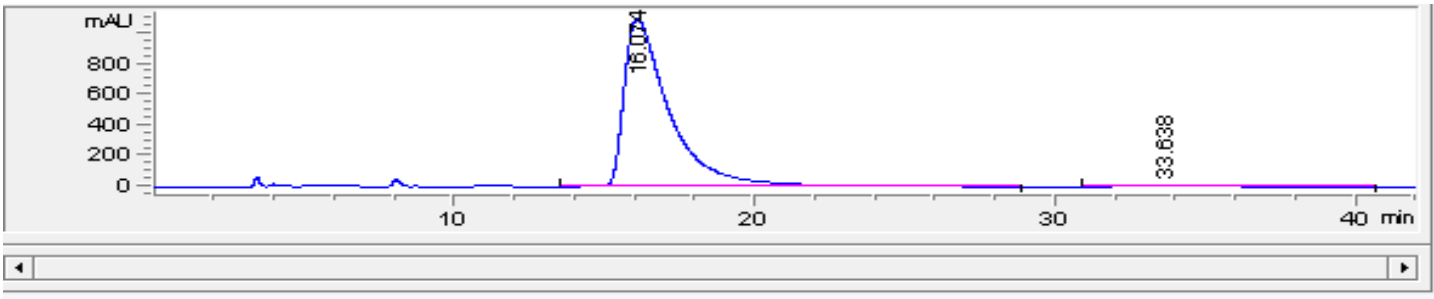

\begin{tabular}{|c|c|c|c|c|c|c|} 
\# & \multicolumn{1}{c}{ Time } & \multicolumn{1}{c}{ Area } & Height & \multicolumn{1}{c|}{ Width } & \multicolumn{1}{c}{ Area\% $\%$} & Symmetry \\
\hline 1 & 16.074 & 113786.8 & 1092.8 & 1.5228 & 98.907 & 0.376 \\
\hline 2 & 33.638 & 1257.2 & 6.5 & 2.323 & 1.093 & 0.635 \\
\hline
\end{tabular}


${ }^{1} \mathrm{H}$ and ${ }^{13} \mathrm{C}$ NMR of $3 \mathrm{r}$
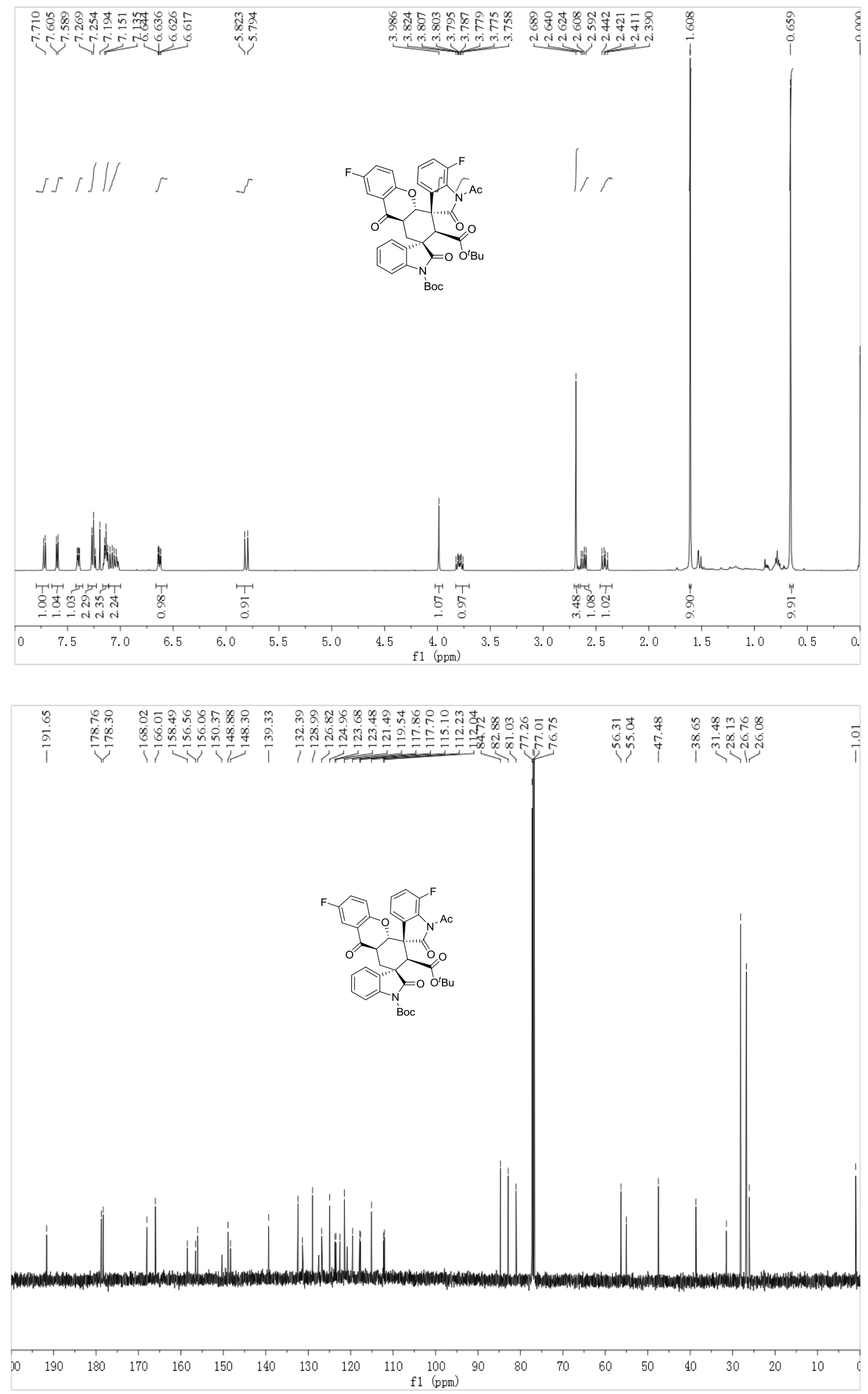


\section{${ }^{19}$ F NMR of $3 r$}

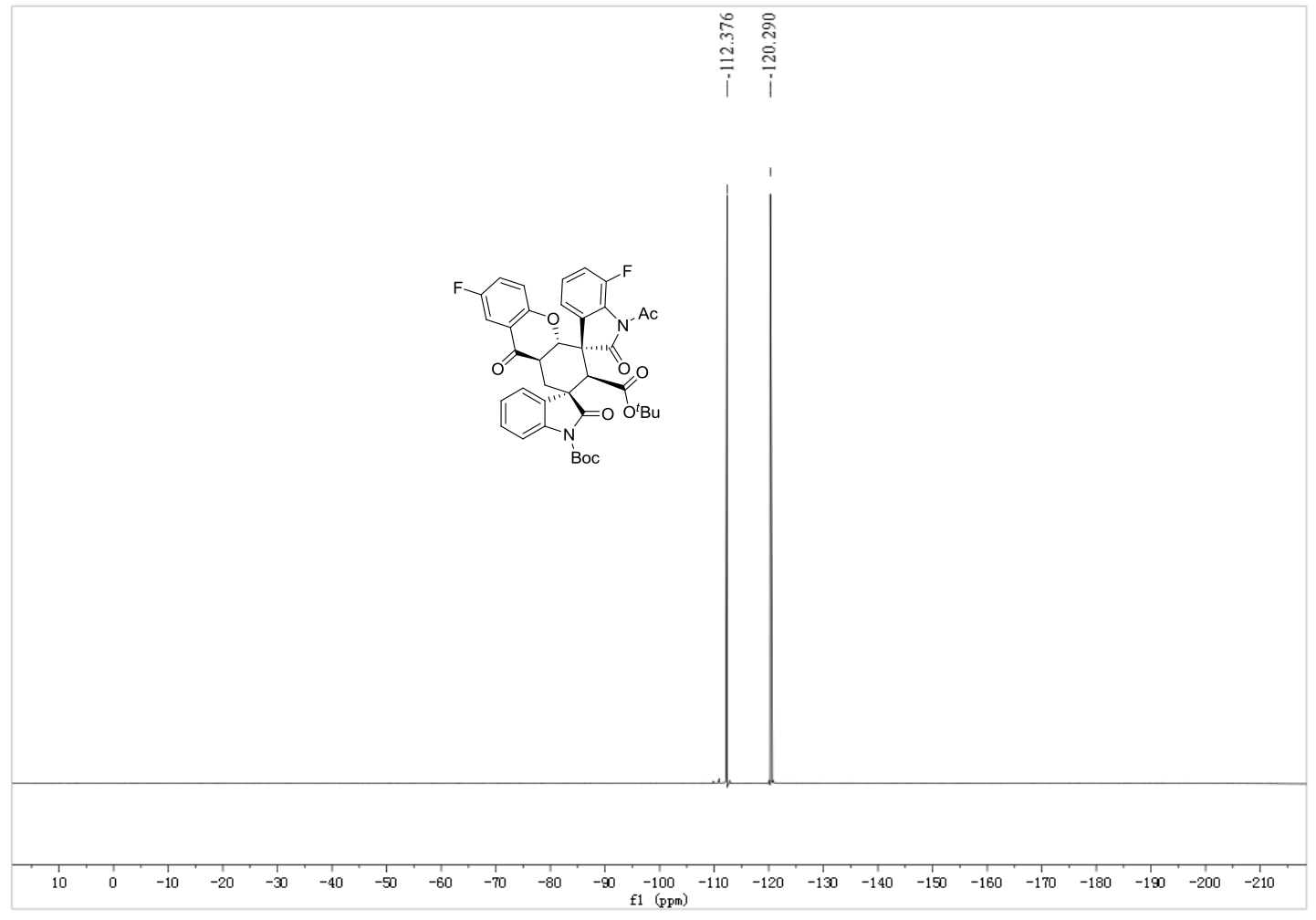

\section{HPLC of 3r}
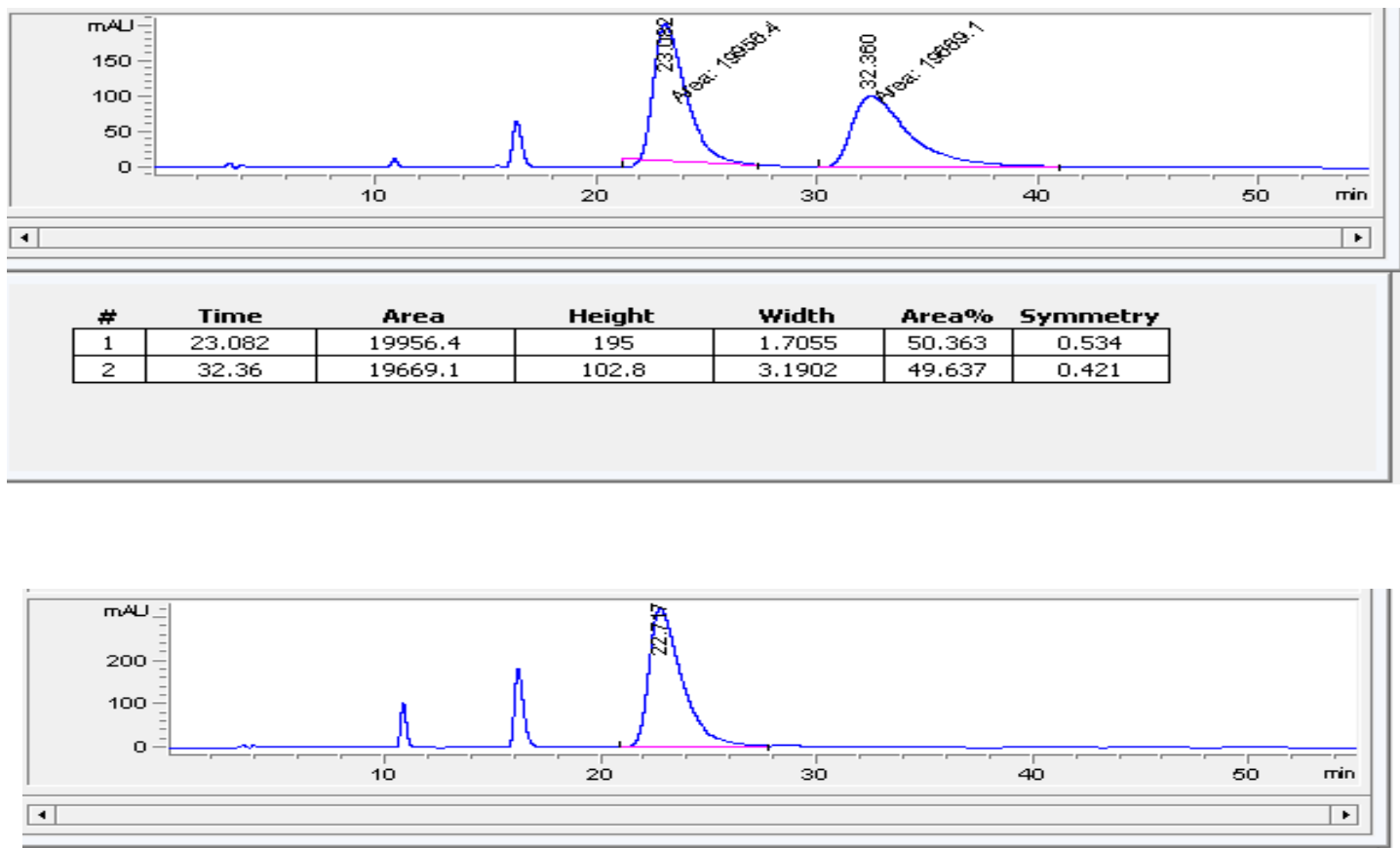

\begin{tabular}{|c|c|c|c|c|c|c|}
\hline$\#$ & Time & Area & Height & Width & Area\% & Symmetry \\
\hline 1 & 22.717 & 33768.8 & 318.2 & 1.5862 & 100.000 & 0.486 \\
\hline
\end{tabular}


${ }^{1} \mathrm{H}$ and ${ }^{13} \mathrm{C}$ NMR of $3 \mathrm{~s}$

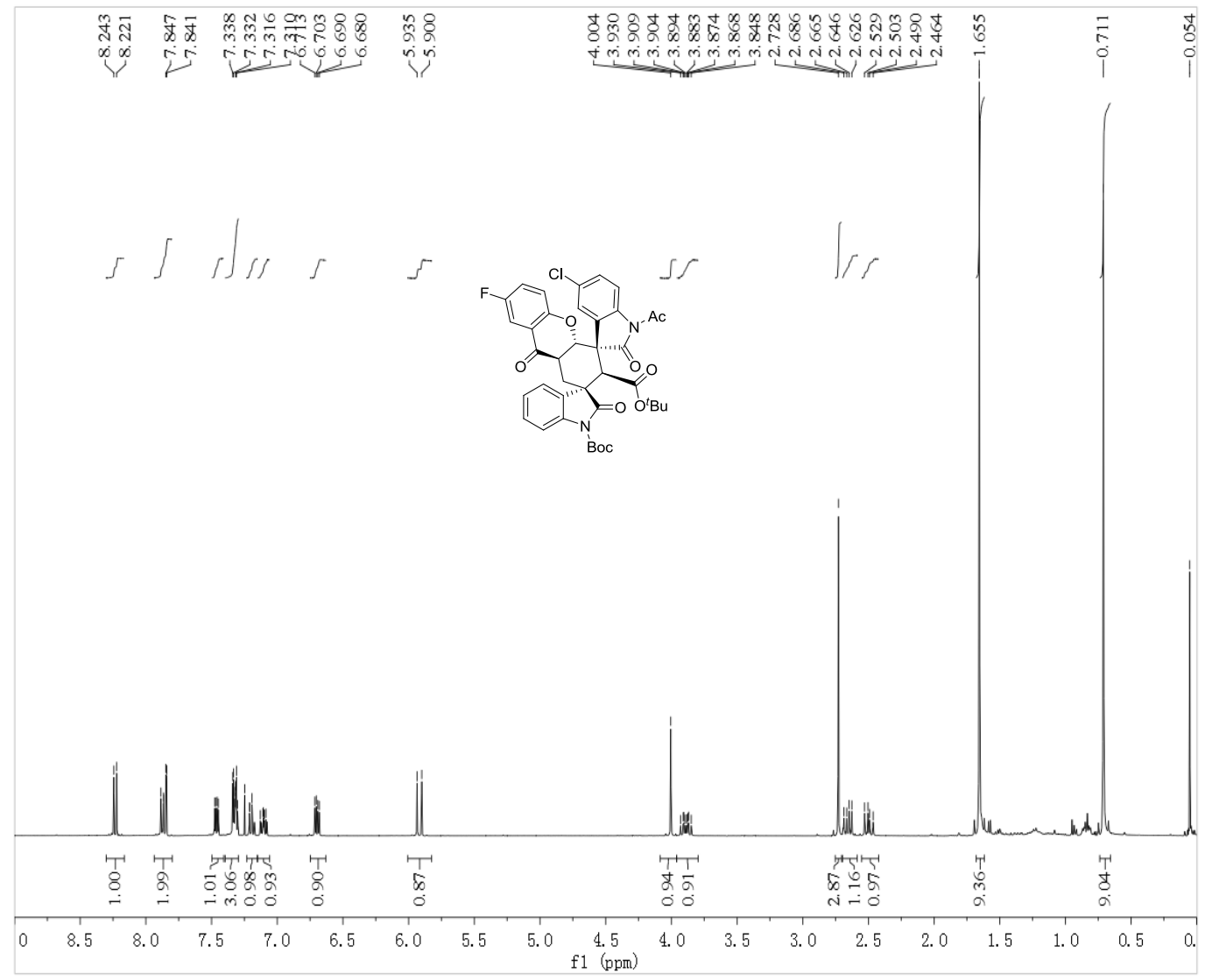

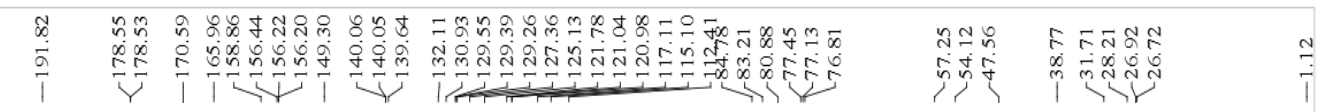

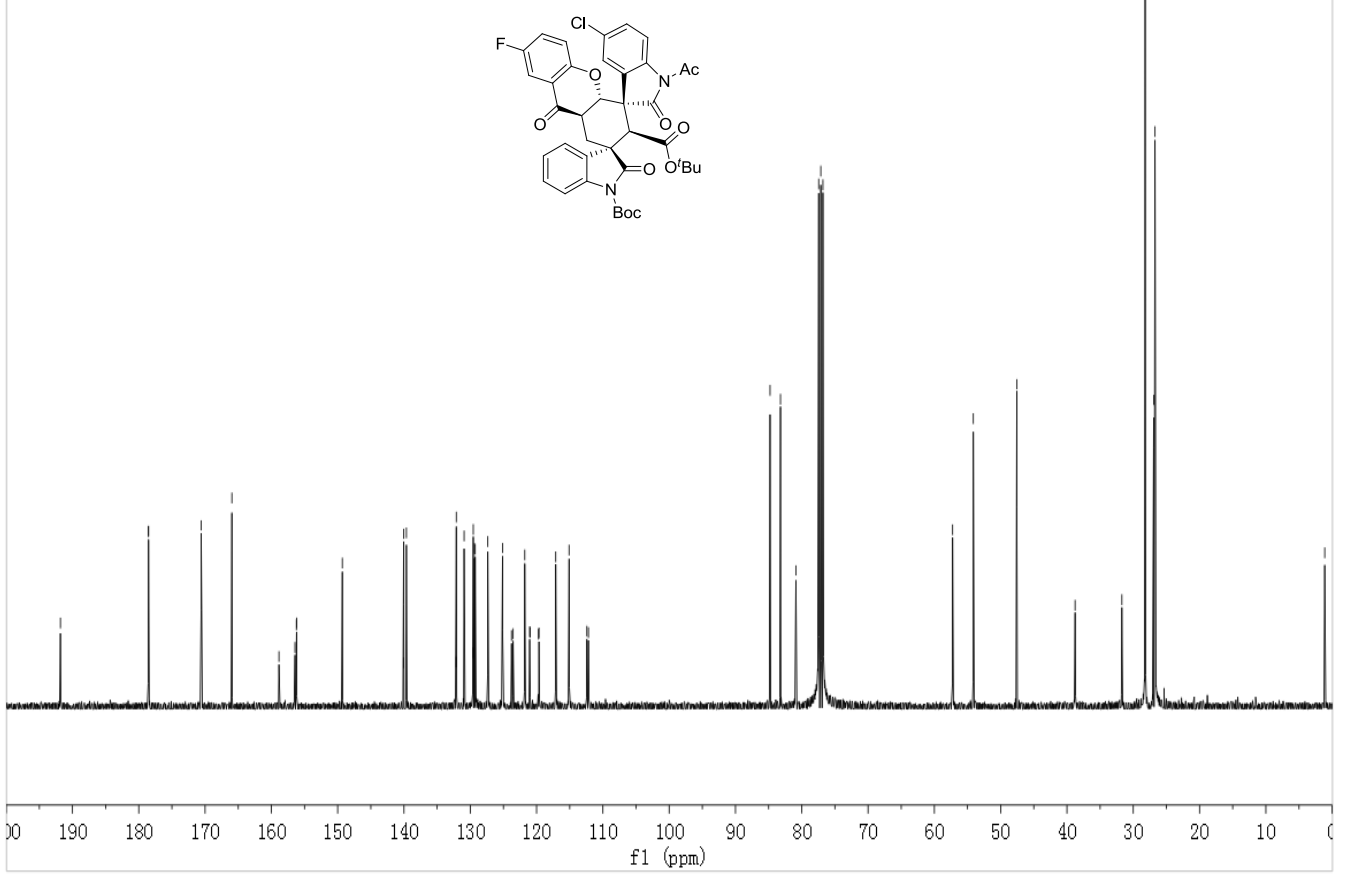




\section{${ }^{19}$ F NMR of $3 \mathrm{~s}$}

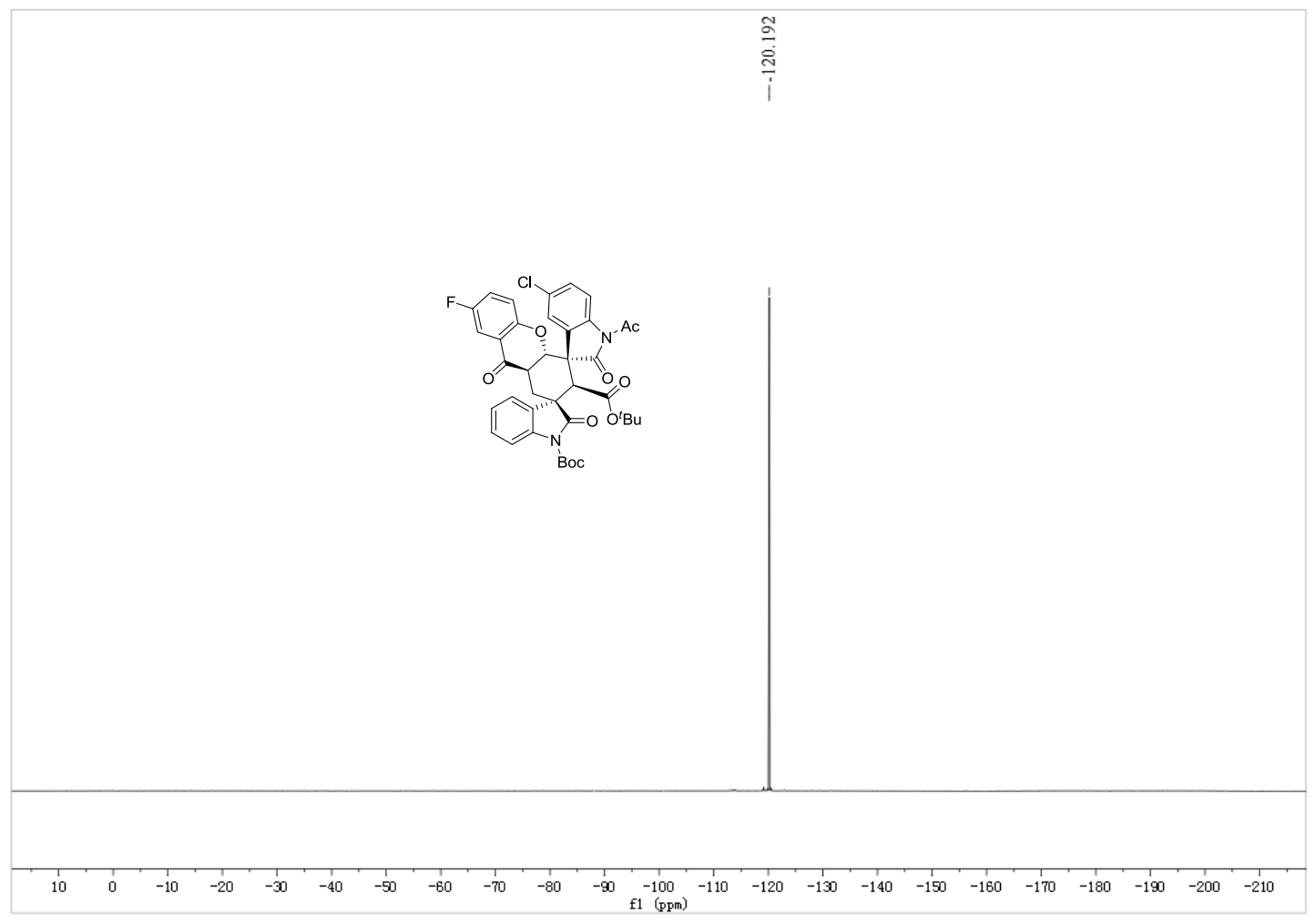

\section{HPLC of 3s}

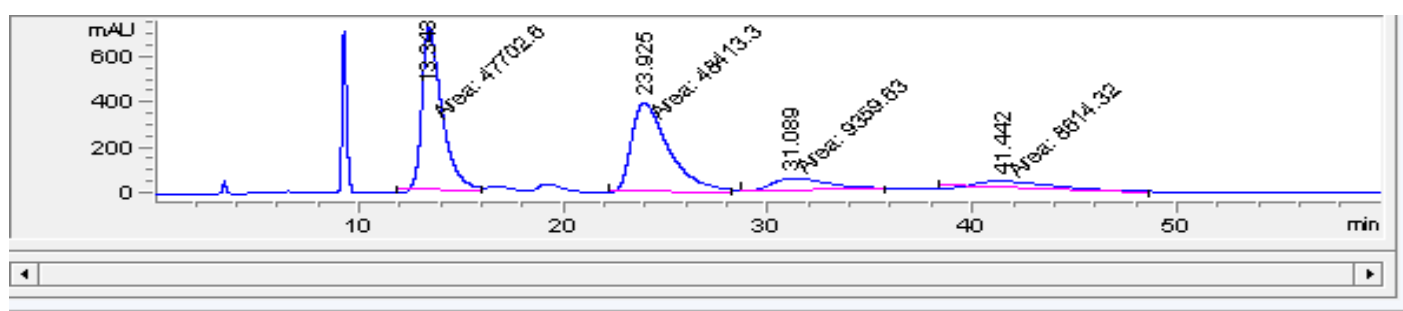

\begin{tabular}{|c|c|c|c|c|c|c|}
\hline \# & Time & Area & Height & Width & Area\% & Symmetry \\
\hline 1 & 13.343 & 47702.6 & 711 & 1.1182 & 41.811 & 0.524 \\
\hline 2 & 23.925 & 48413.3 & 388.1 & 2.0793 & 42.434 & 0.495 \\
\hline 3 & 31.089 & 9359.6 & 52.1 & 2.9963 & 8.204 & 0.499 \\
\hline 4 & 41.442 & 8614.3 & 31.5 & 4.5608 & 7.550 & 0.338 \\
\hline
\end{tabular}

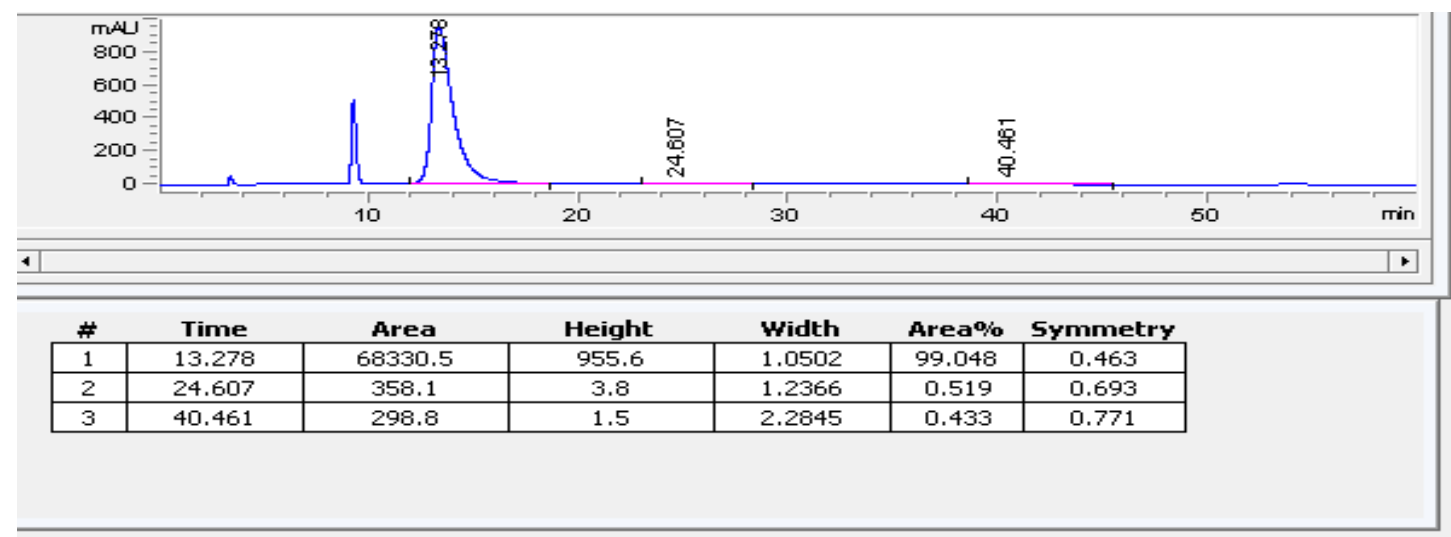


${ }^{1} \mathrm{H}$ and ${ }^{13} \mathrm{C}$ NMR of $3 \mathrm{t}$

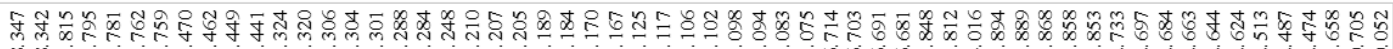

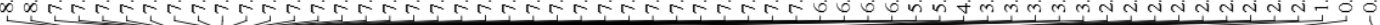

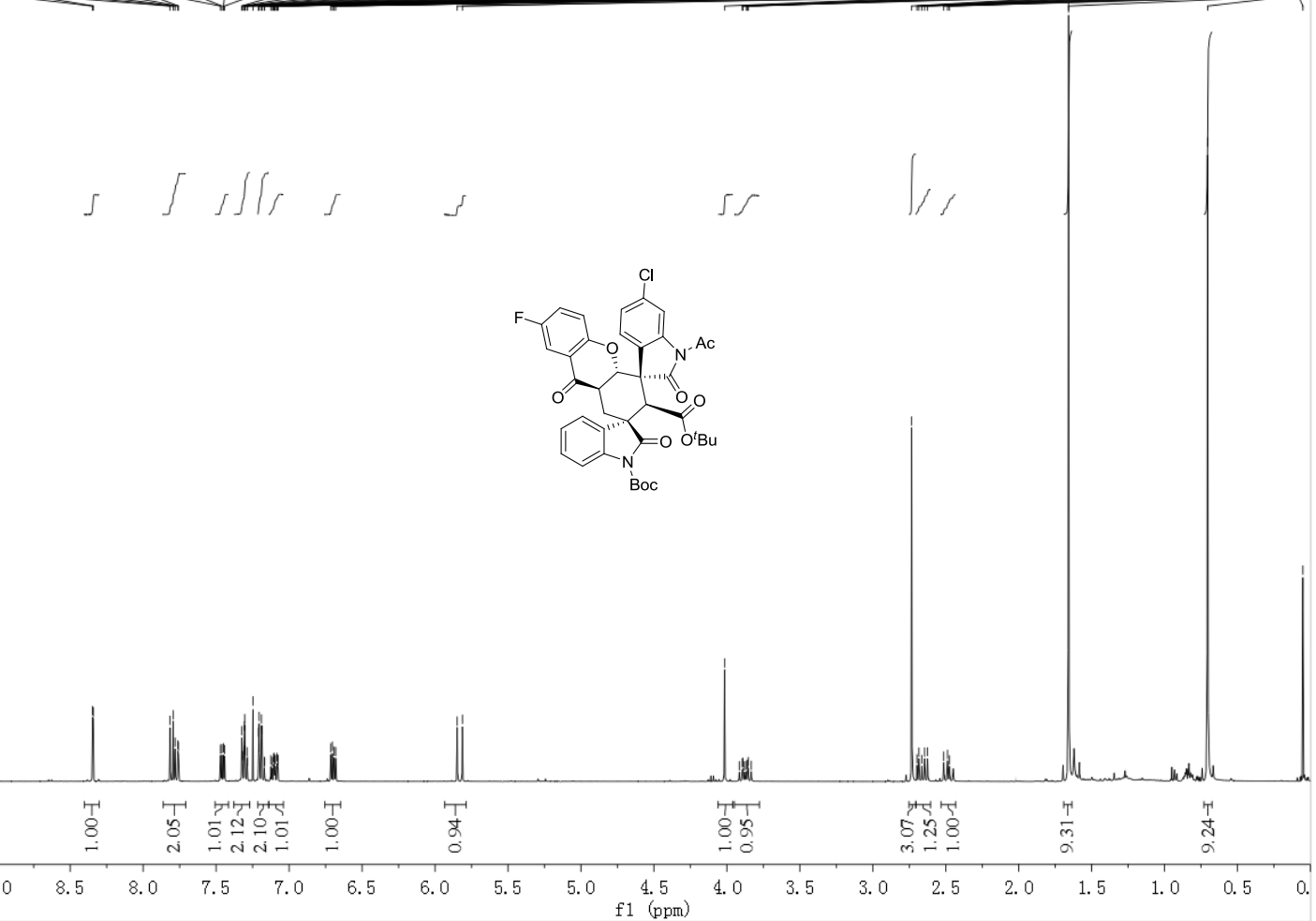

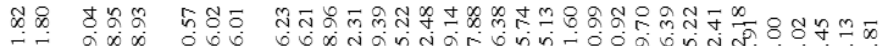

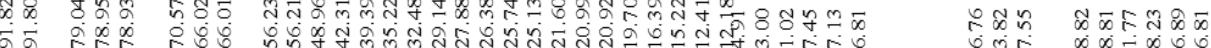

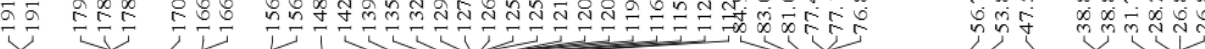

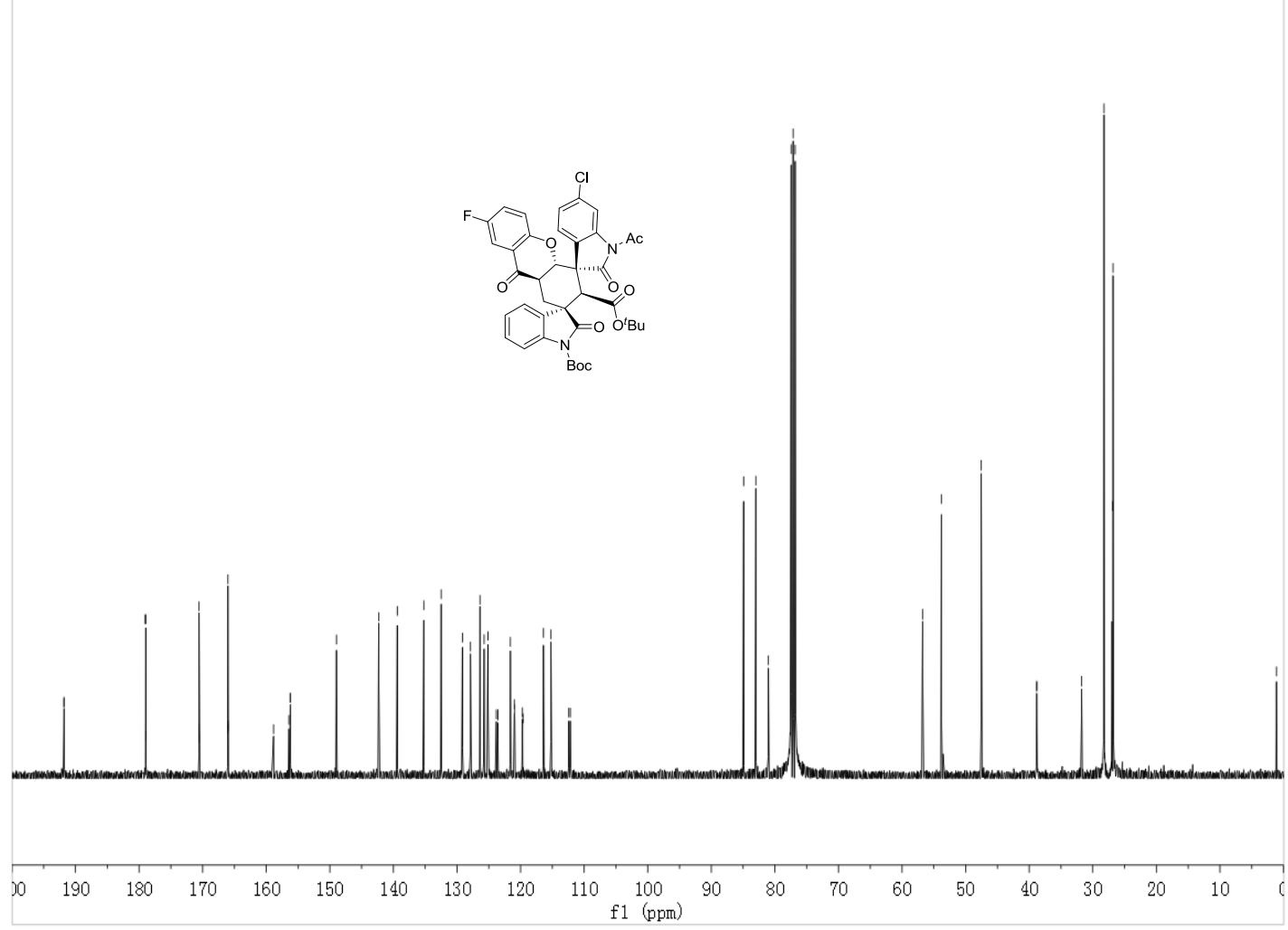




\section{${ }^{19}$ F NMR of $3 t$}

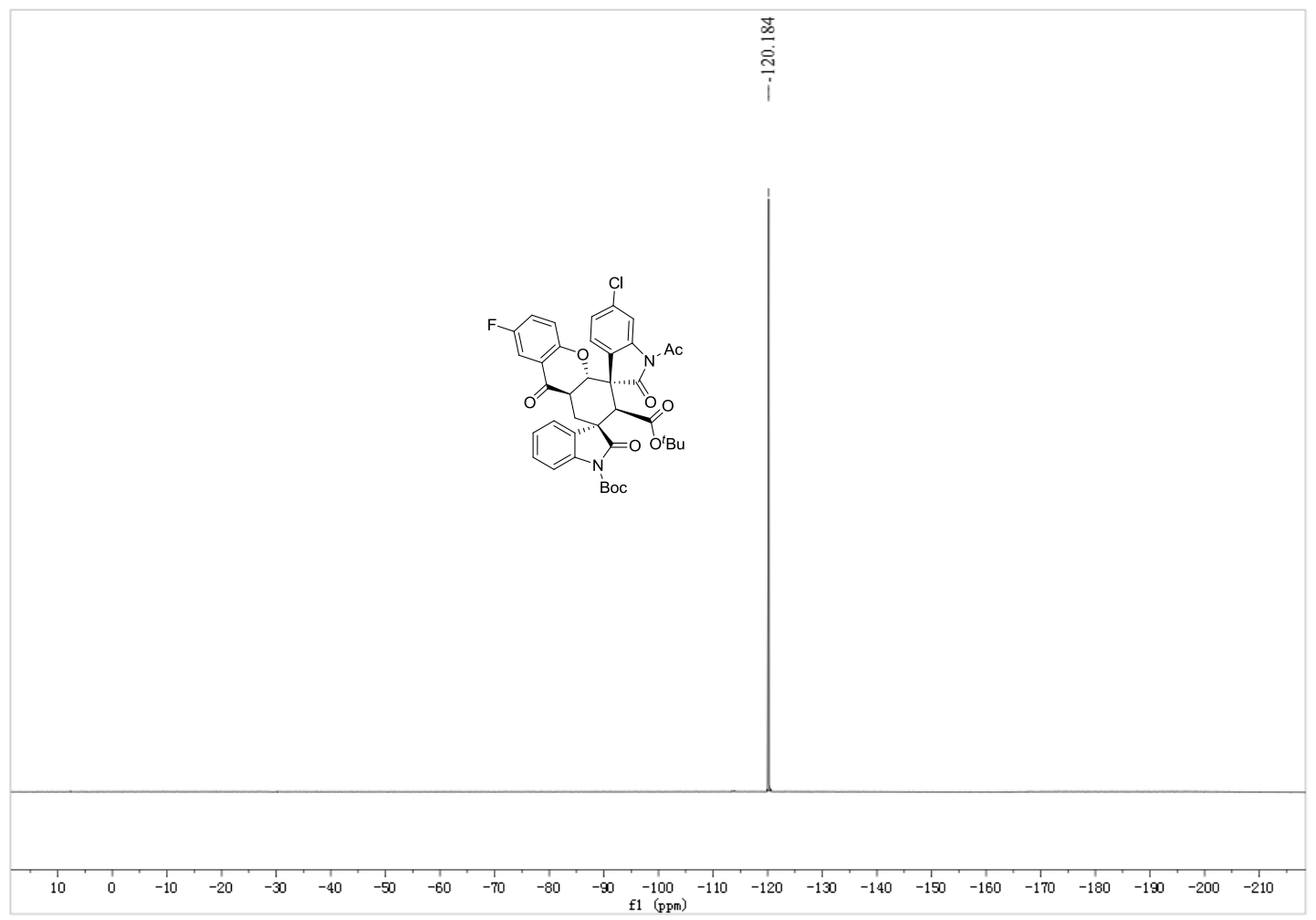

HPLC of $3 t$
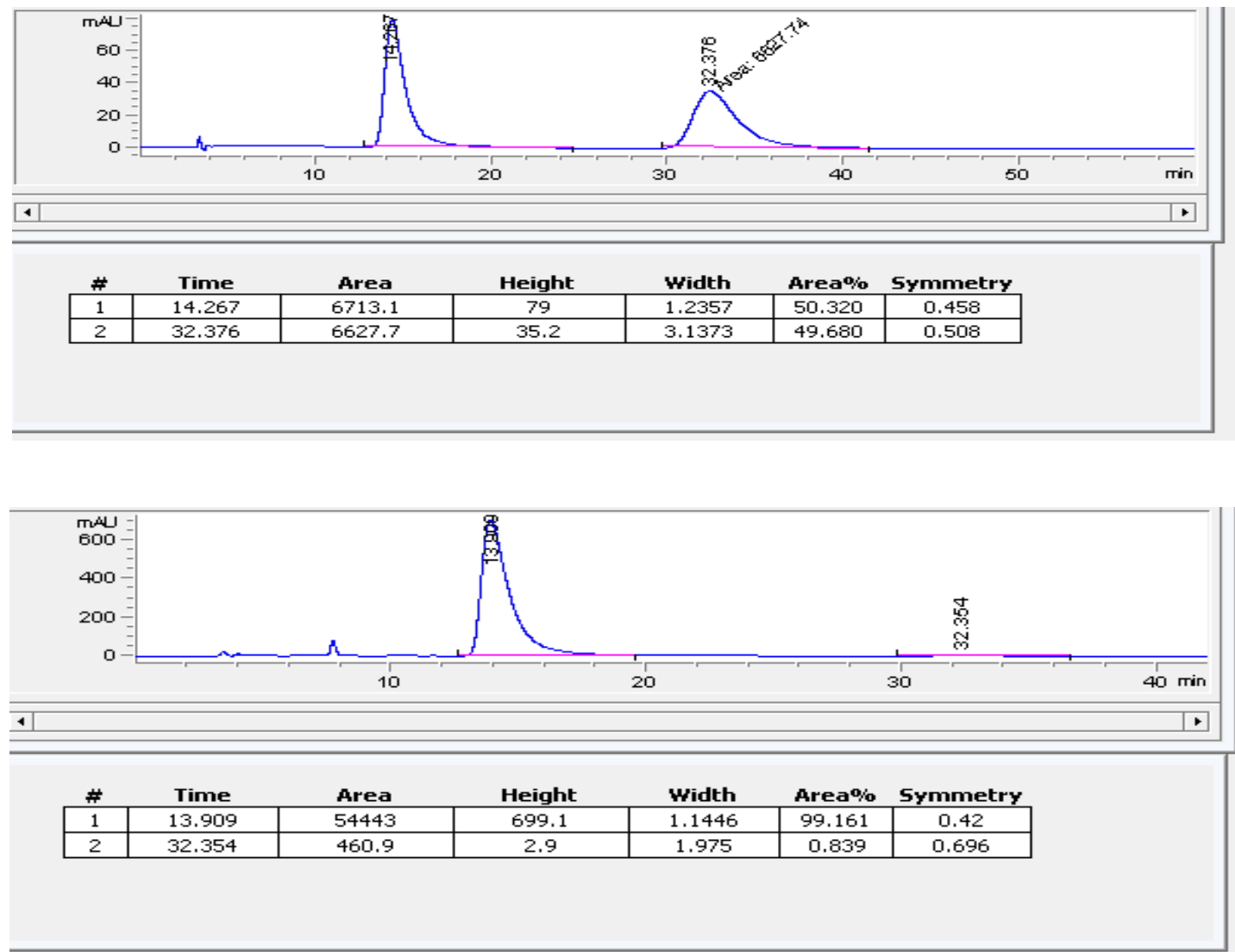
${ }^{1} \mathrm{H}$ and ${ }^{13} \mathrm{C}$ NMR of $3 \mathrm{u}$

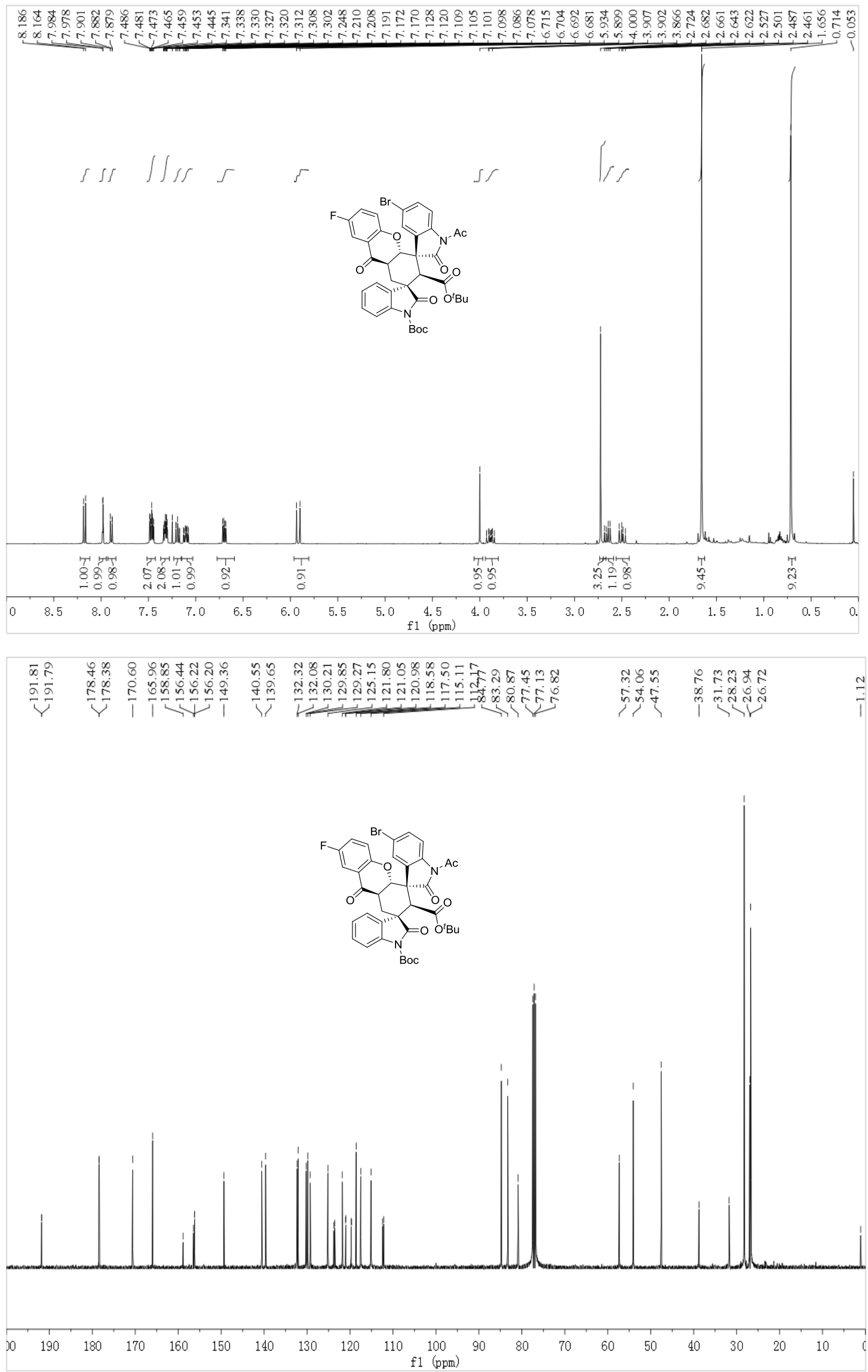




\section{${ }^{19}$ F NMR of $3 u$}

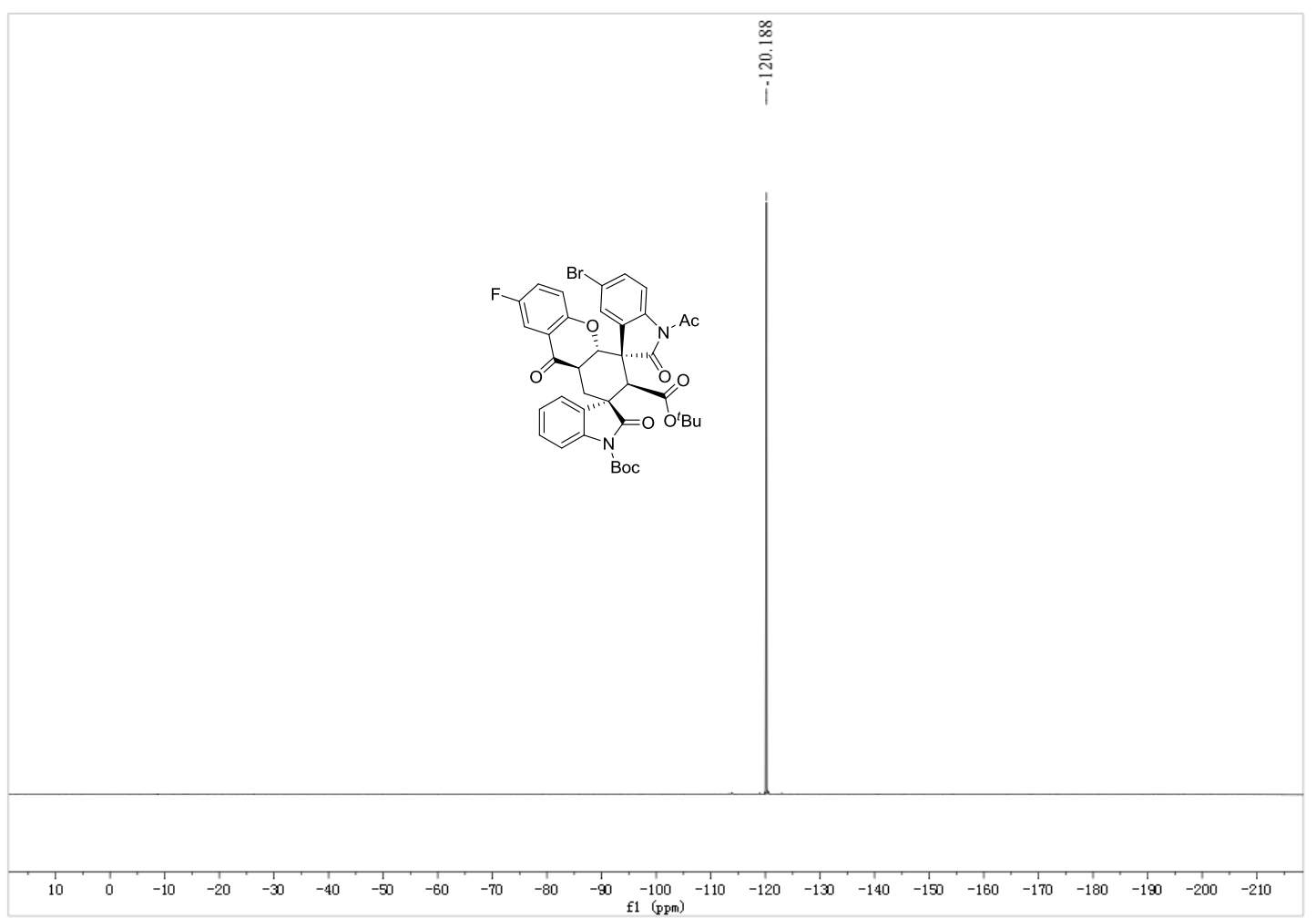

HPLC of $3 u$

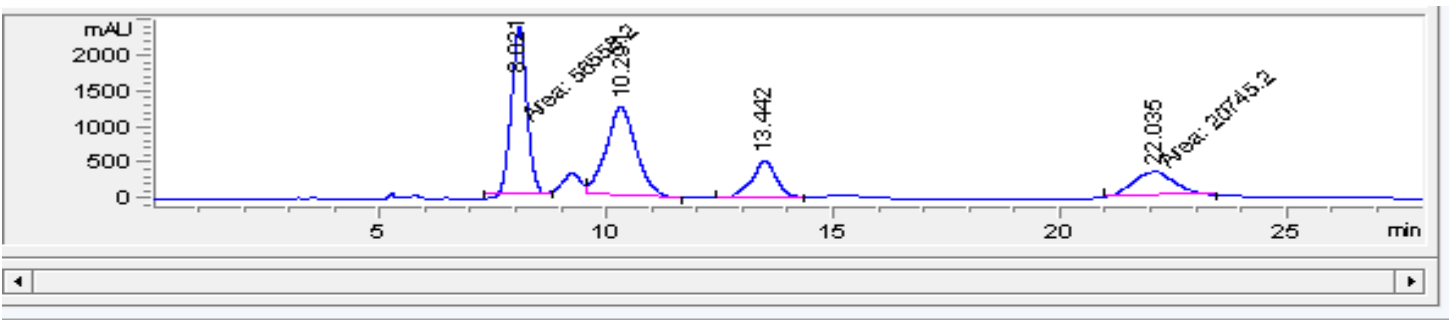

\begin{tabular}{|c|c|c|c|c|c|c|}
\multicolumn{1}{l}{ \# } & \multicolumn{1}{c}{ Time } & \multicolumn{1}{c}{ Area } & Height & \multicolumn{1}{c}{ Width } & \multicolumn{1}{c}{ Area\% $\%$ Symmetry } \\
\hline 1 & 8.021 & 56558.2 & 2354.3 & 0.4004 & 36.494 & 0.942 \\
\hline 2 & 10.291 & 57488.3 & 1252.5 & 0.6795 & 37.094 & 0.922 \\
\hline 3 & 13.442 & 20188.2 & 527.9 & 0.5502 & 13.026 & 1.115 \\
\hline 4 & 22.035 & 20745.2 & 333.7 & 1.0361 & 13.386 & 0.906 \\
\hline
\end{tabular}

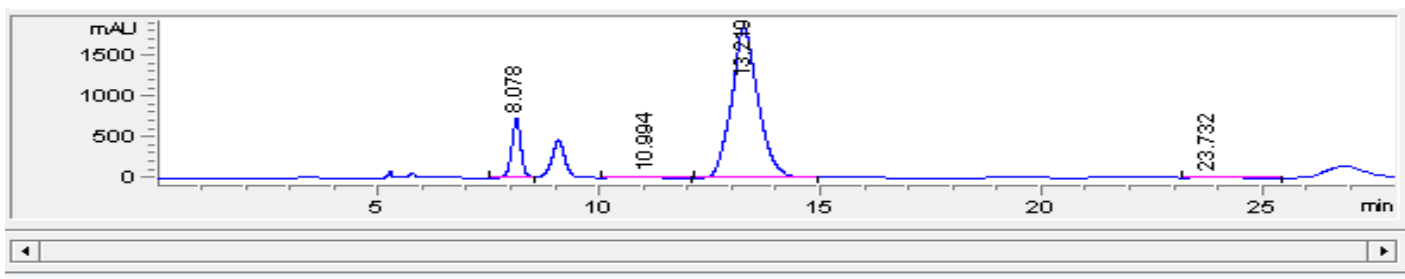

\begin{tabular}{|c|c|c|c|c|c|c|}
\hline \# & Time & Area & Height & Width & Area\% & Symmetry \\
\hline 1 & 8.078 & 10428.3 & 720.9 & 0.2188 & 11.845 & 1.037 \\
\hline 2 & 10.994 & 388.6 & 9.7 & 0.5609 & 0.441 & 1.398 \\
\hline 3 & 13.219 & 76901.1 & 1855 & 0.5997 & 87.350 & 0.793 \\
\hline 4 & 23.732 & 319.7 & 4.6 & 0.9593 & 0.363 & 0.475 \\
\hline
\end{tabular}


${ }^{1} \mathrm{H}$ and ${ }^{13} \mathrm{C}$ NMR of $3 \mathrm{v}$
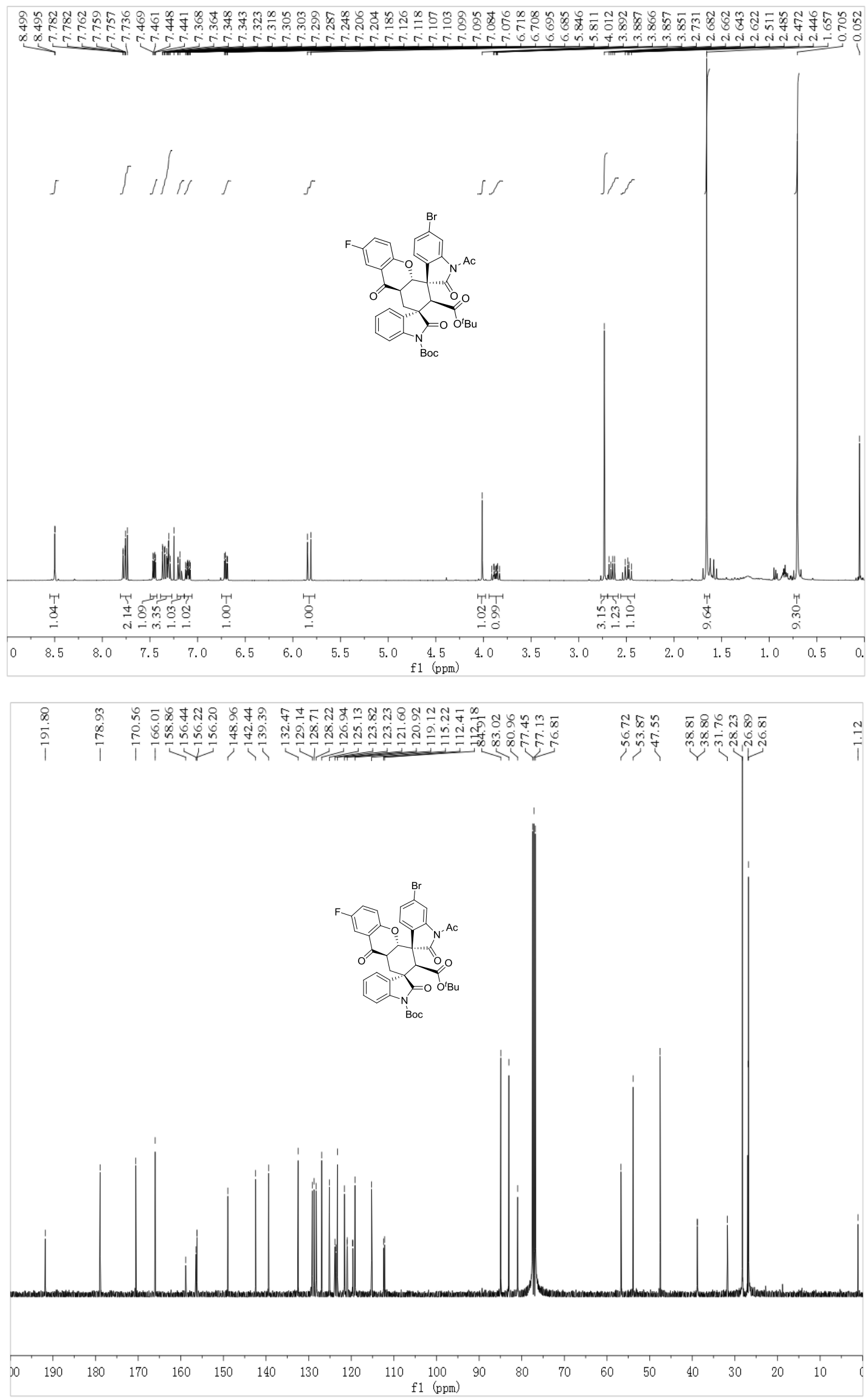


\section{${ }^{19}$ F NMR of $3 \mathrm{v}$}

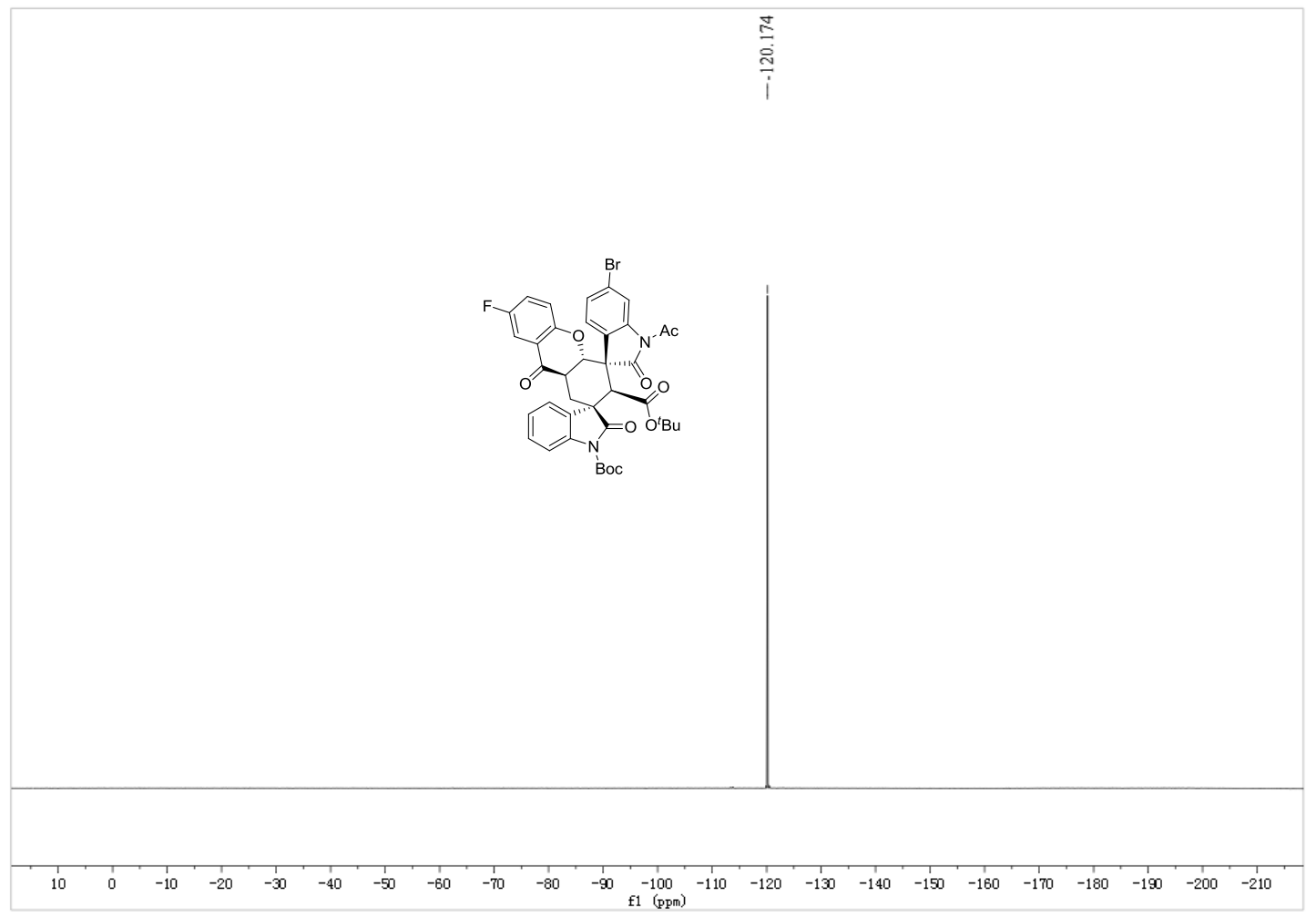

\section{HPLC of $3 v$}
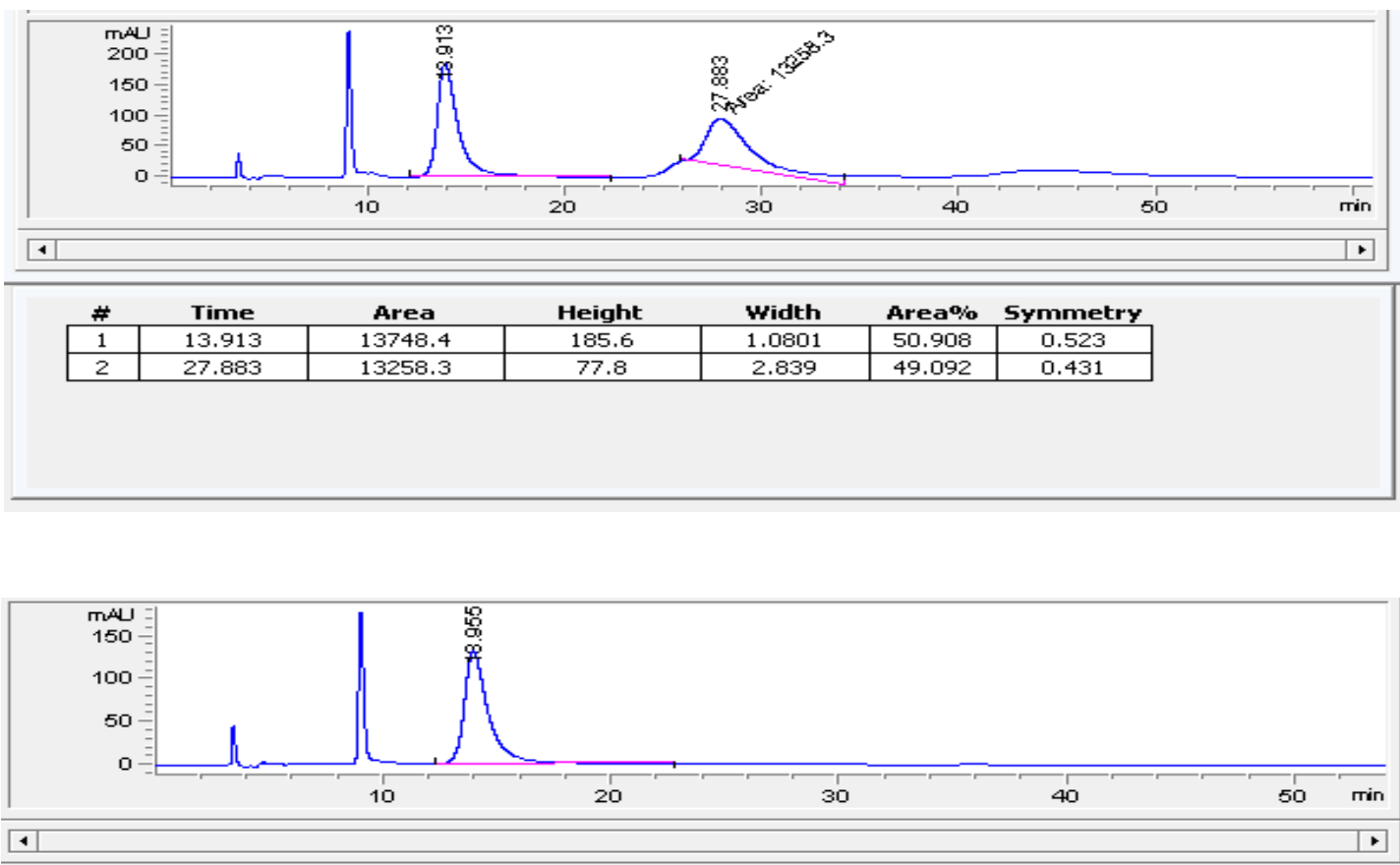

\begin{tabular}{|c|c|c|c|c|c|c|} 
\# & \multicolumn{1}{c}{ Time } & \multicolumn{1}{c}{ Area } & Height & Width & \multicolumn{2}{c|}{ Area\% } \\
\hline 1 & 13.955 & 9923.4 & 132.2 & 1.0936 & 100.000 & 0.527 \\
\hline
\end{tabular}




\section{${ }^{1} \mathrm{H}$ and ${ }^{13} \mathrm{C}$ NMR of $3 w$}
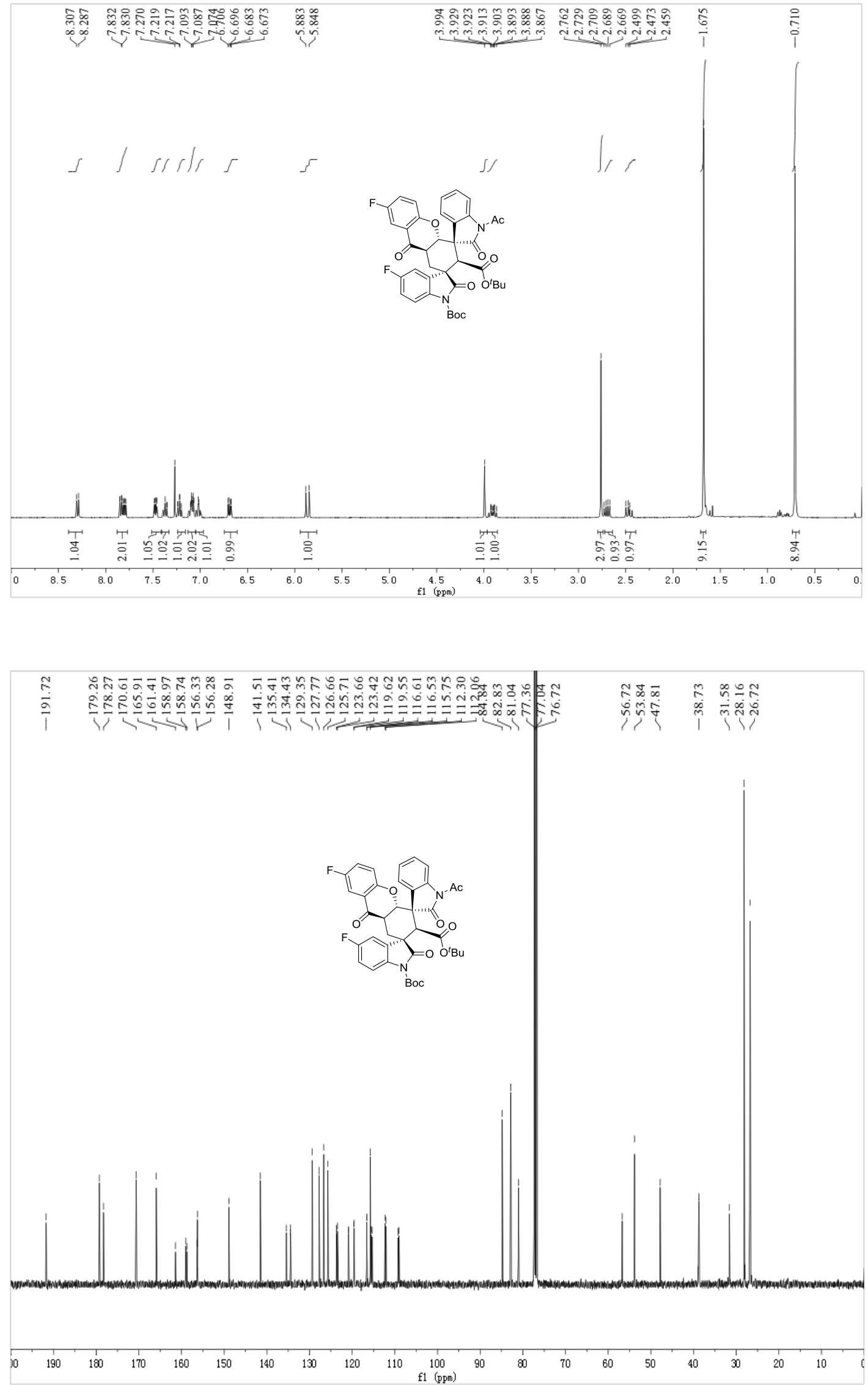
${ }^{19}$ F NMR of 3w

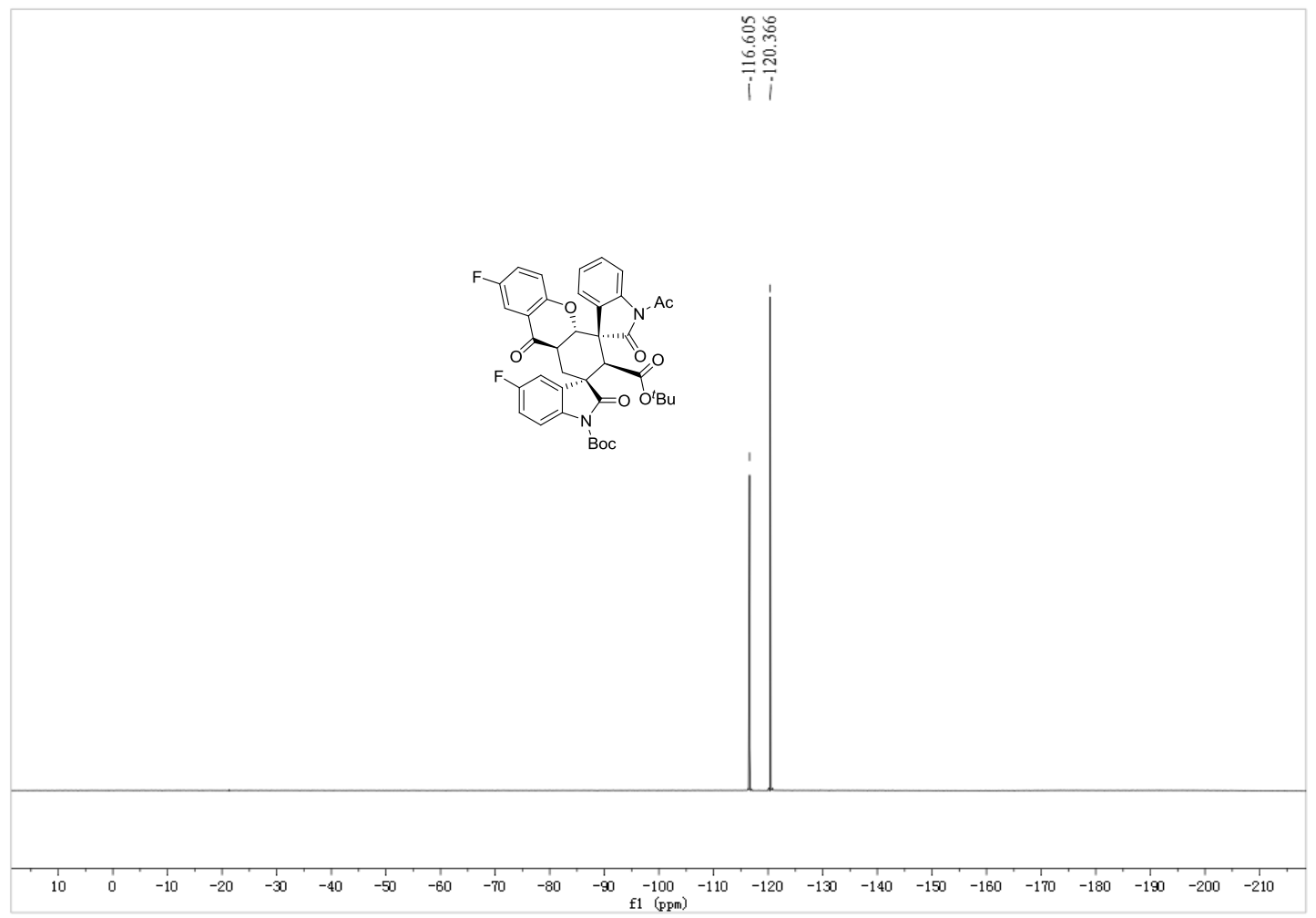

\section{HPLC of 3w}

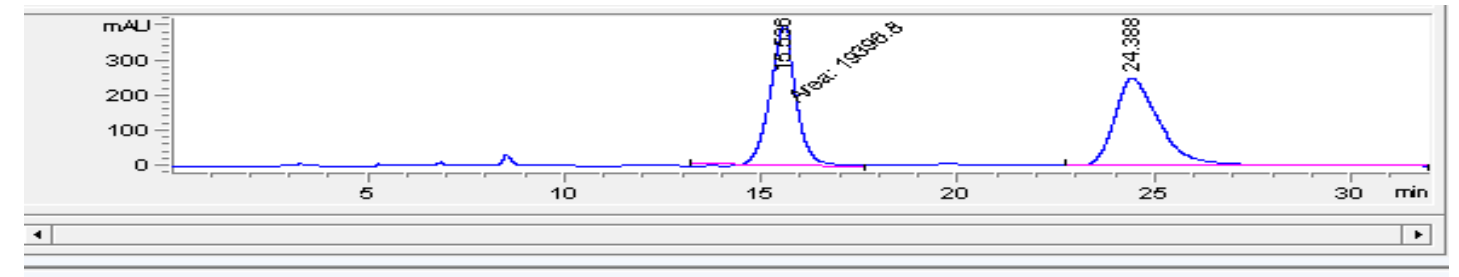

\begin{tabular}{|c|c|c|c|c|c|c|} 
\# & \multicolumn{1}{c}{ Time } & Area & Height & \multicolumn{1}{c}{ Width } & \multicolumn{1}{c}{ Area\% $\%$ Symmetry } \\
\hline 1 & 15.536 & 19396.8 & 403.3 & 0.8015 & 49.701 & 0.992 \\
\hline 2 & 24.388 & 19630 & 249.4 & 1.1584 & 50.299 & 0.575 \\
\hline
\end{tabular}

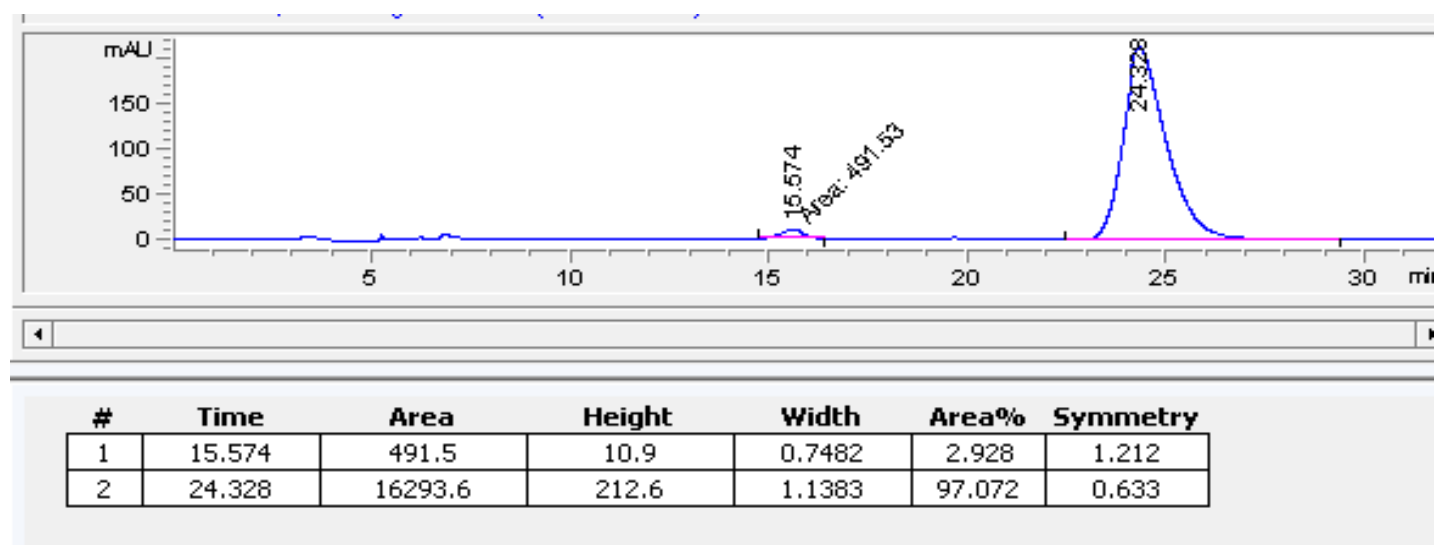


${ }^{1} \mathrm{H}$ and ${ }^{13} \mathrm{C}$ NMR of $3 \mathrm{x}$
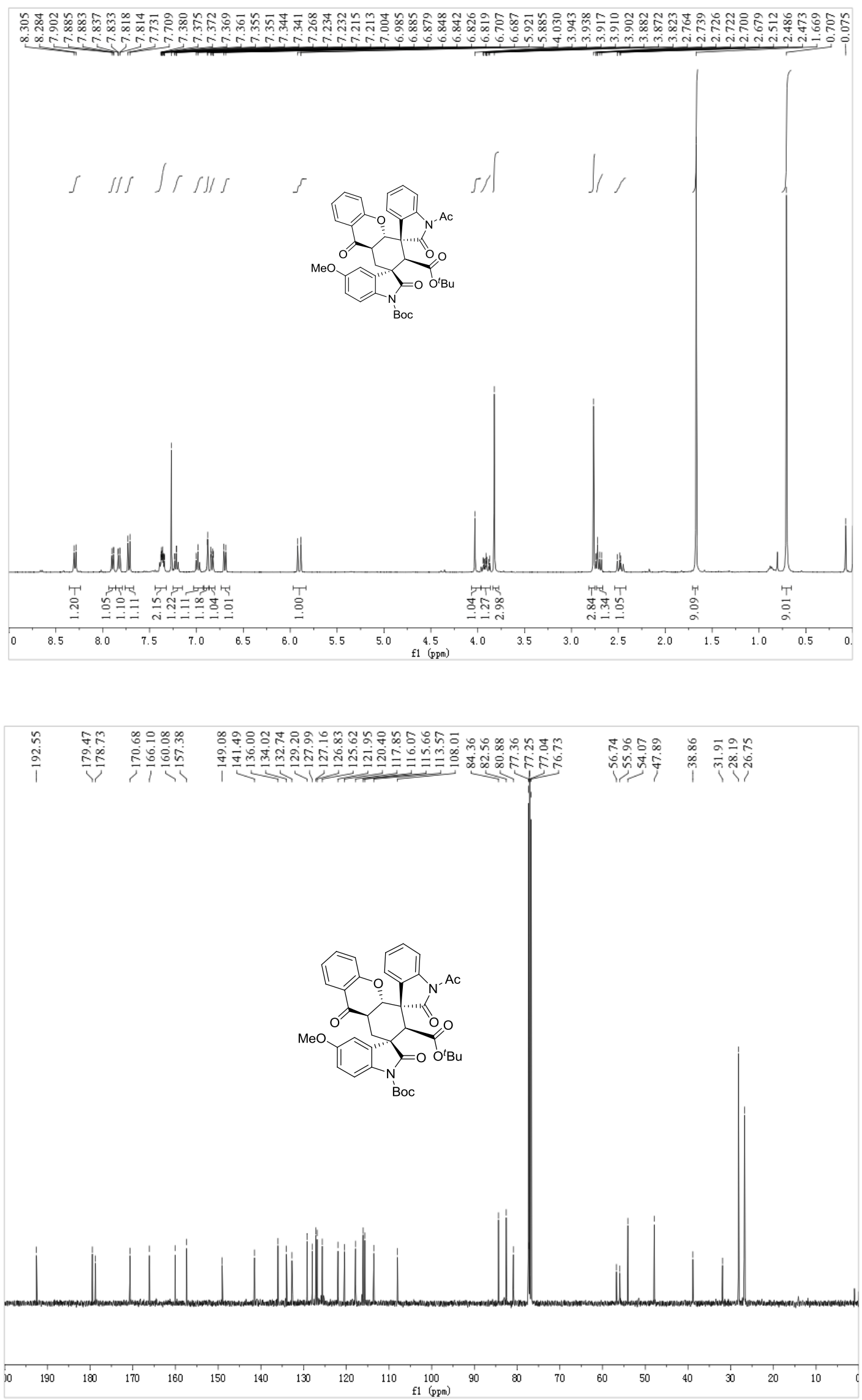
HPLC of 3x

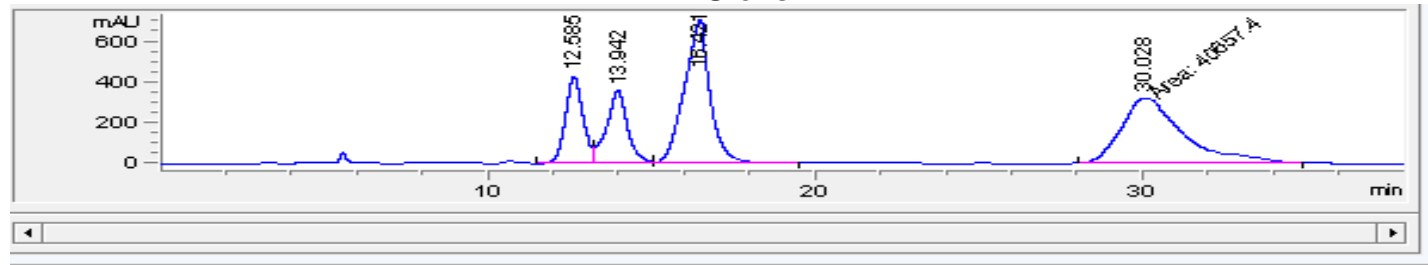

\begin{tabular}{|c|c|c|c|c|c|c|}
\hline \# & Time & Area & Height & Width & Area\% & Symmetry \\
\hline 1 & 12.585 & 15705.4 & 428.4 & 0.5477 & 14.078 & 0.875 \\
\hline 2 & 13.942 & 15543.3 & 358.6 & 0.6176 & 13.932 & 1.123 \\
\hline 3 & 16.421 & 39655.2 & 704.4 & 0.8048 & 35.546 & 1.205 \\
\hline 4 & 30.028 & 40657.4 & 318.3 & 2.1287 & 36.444 & 0.574 \\
\hline
\end{tabular}

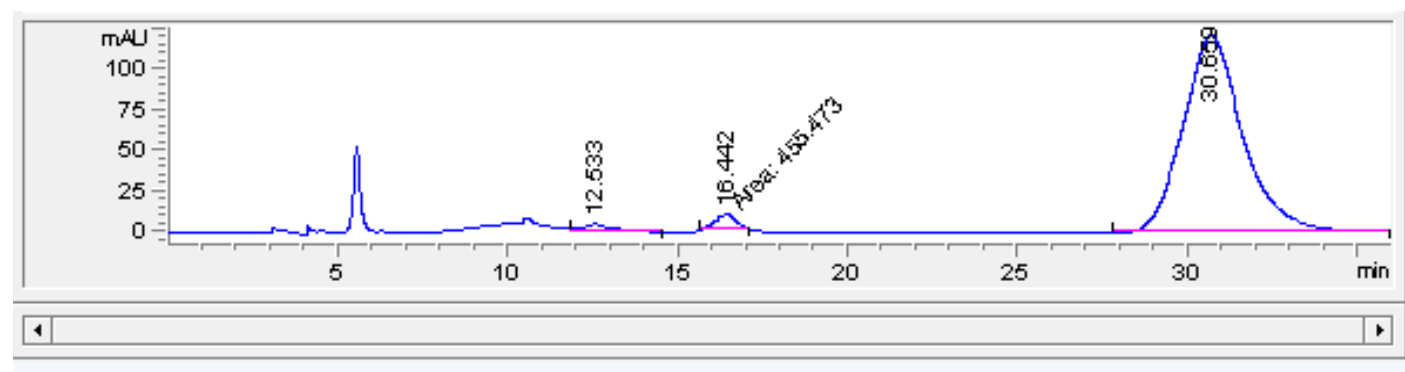

\begin{tabular}{|c|c|c|c|c|c|c|} 
\# & \multicolumn{1}{c}{ Time } & \multicolumn{1}{c}{ Area } & Height & \multicolumn{1}{c}{ Width } & \multicolumn{2}{c|}{ Area\% $\%$ Symmetry } \\
\hline 1 & 12.533 & 329.8 & 4.8 & 0.9375 & 2.132 & 0.655 \\
\hline 2 & 16.442 & 455.5 & 9.8 & 0.7724 & 2.945 & 1.259 \\
\hline 3 & 30.659 & 14683.1 & 120.6 & 1.7689 & 94.923 & 0.817 \\
\hline
\end{tabular}

